Andrews University

Digital Commons @ Andrews University

Master's Theses

Graduate Research

1976

\title{
Micro-Level Enrollment Projections: A Study Involving the Parish of Hanover, Jamaica
}

\author{
E G. Marshalleck \\ Andrews University
}

Follow this and additional works at: https://digitalcommons.andrews.edu/theses

Part of the Elementary Education Commons

\section{Recommended Citation}

Marshalleck, E G., "Micro-Level Enrollment Projections: A Study Involving the Parish of Hanover, Jamaica" (1976). Master's Theses. 161.

https://dx.doi.org/10.32597/theses/161

https://digitalcommons.andrews.edu/theses/161

This Thesis is brought to you for free and open access by the Graduate Research at Digital Commons @ Andrews University. It has been accepted for inclusion in Master's Theses by an authorized administrator of Digital Commons@ Andrews University. For more information, please contact repository@andrews.edu. 


\title{
Andrews University
}

School of Graduate Studies

\author{
MICRO-LEVEL ENROLLMENT PROJECTIONS - A STUDY \\ INVOLVING THE PARISH OF HANOVER, JAMAICA
}

\author{
A Thesis \\ Presented in Partial Fulfillment \\ of the Requirements for the Degree \\ Master of Arts
}

by

E. G. Marshalleck

May 1976

Approved 


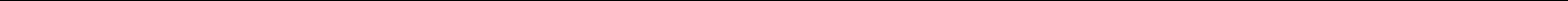




\section{ABSTRACT OF GRADUATE STUDENT RESEARCH}

Thesis

Andrews University

Department of Education

Title: MICRO-LEVEL ENROLLMENT PROJECTIONS - A STUDY INVOLVING THE PARISH OF HANOVER, SAMAICA

Name of researcher: E. G. Marshalleck

Name and title of faculty advisers: Robert J. Cruise, Ph.D., Chairman Rudolf E. Klimes, Ph.D.

Marion J. Merchant, Ph.D.

Date completed: May 1976

Problem

While global forecasts of pupil populations are vital to educational planning, projections at micro-level are even more pertinent to detailed planning.

In Jamaica, the need for local level forecasts becomes particularly apparent every September, when there is great pressure from would-be pupils on urban school accommodation.

\section{Method}

The review of 1iterature was aimed at identifying projection methods feasible for micro-level applications. The following three methods, identified by Webster (1969) as about the most efficient of seventeen ratio and regression methods of pupil projection which he investigated, were selected for testing on data for the parish of Hanover, Jamaica: Transition Analysis, Time Analysis, and Cohort Survival. Transition Analysis and Cohort Survival utilize birth and 
enrollment data as predictors. Time Analysis utilizes time and enrollment. The research also involved the creation of fourteen "school districts" in the parish on the basis of physiographic features. Districts were also classified according to previous population change.

\section{Results}

Utilizing birth data for 1958/59-1966/67 and enrollment data for 1965/66-1973/74, two sets of projections based on time spans of 8-7 and 6-5 years were made for each of the three methods in seven districts. These projections were gauged against actual enrollment for $1973 / 74$, the third succeeding and immediately succeeding year for the above periods. Both Time Analysis and Cohort Survival yielded better estimates than Transition Analysis with Time Analysis having the edge over Cohort Survival. Since Transition Analysis fell short of expectations and since it was suspected that small, erratic figures were the cause, the methods were tested on total figures for the parish. Here Transition Analysis performed best of the three methods.

\section{Conclusion}

Either Time Analysis or Cohort Survival could provide better estimates of future student flows than Transition Analysis in small districts with features like Hanover. On larger, more ordered figures, Transition Analysis might perform best of the three. Tests of the three methods should therefore precede application elsewhere. Statistical methods may usefully be supplemented by other means of estimating population change like the Delphi Technique described in Appendix E.

Further work involving the above techniques is recommended. 


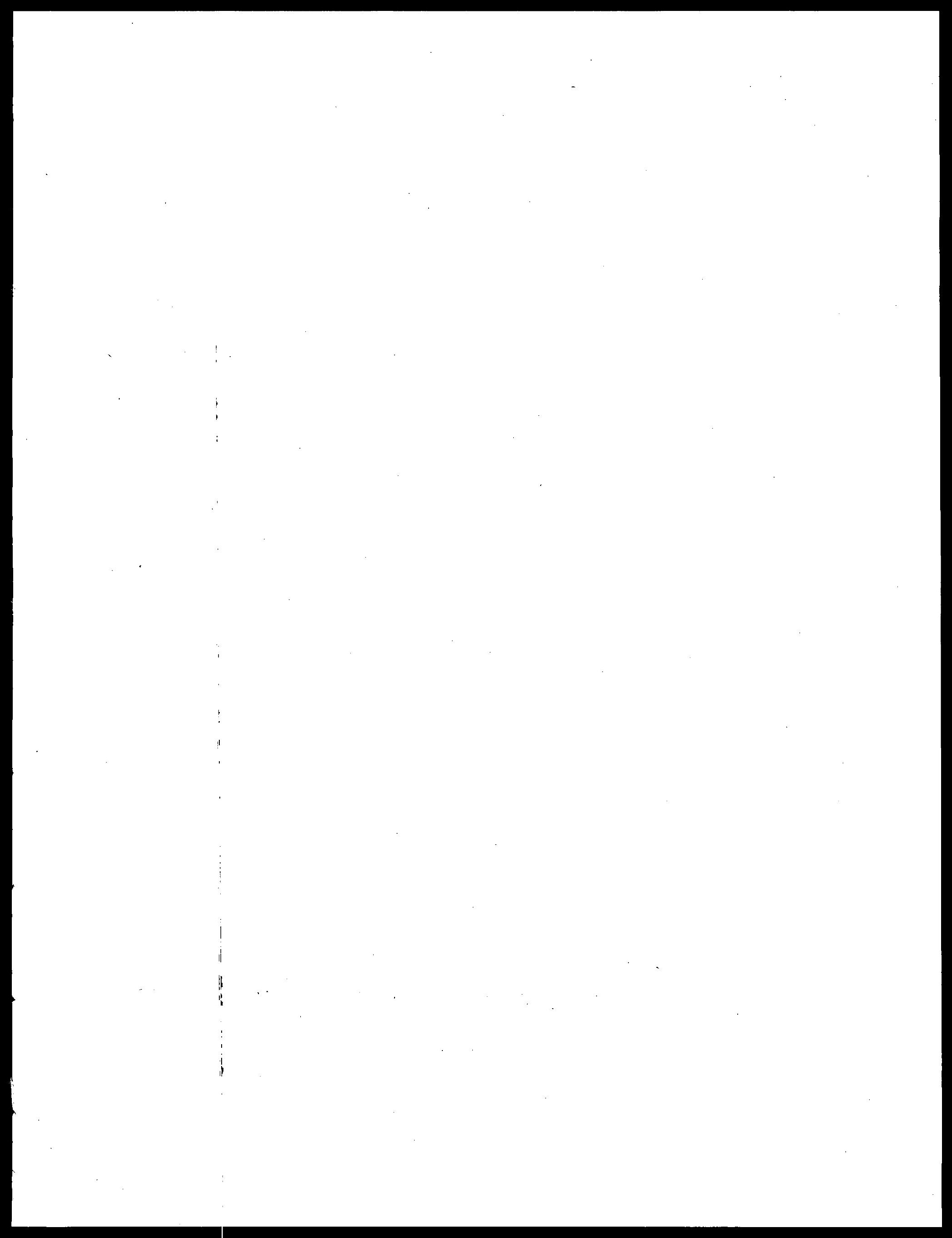




\section{ACKNOWLEDGEMENTS}

Having had every conceivable complication in the gestation and delivery of this work and having survived to see a tangible viable product, I must acknowledge the help and assistance given me by so many persons and organizations.

First, to the Chairman of my committee, Dr. Robert J. Cruise, who gave me expert professional help, quickly making decisions at crucial moments, I tender my grateful thanks. His continuing vote of confidence gave me the mental uplift to continue the study to finality. The other members of my conmittee also deserve and receive my gratitude.

Without the prompt and willing co-operation of public officers in the Ministry of Education, the Department of Statistics and the Island Record office in Jamaica, I could not have secured the data inputs necessary for this study. My sincere thanks go to them and in particular to Mr. Eric Budlall of the Ministry of Education who so often responded to urgent requests for data.

I must also thank Messrs. Dynsdale Spence and K. Harris-Stubbs, Jamaicans, resident in Berrien Springs at the time of the study. The former's intimate knowledge of the parish of Hanover and the 1atter's assistance with handling the data greatly assisted me in deciding on break-downs of the parish into "school districts" and in making data a1locations to these districts.

True friends, Norma Greenidge, Gloria Spence, Gertrude Jordan and Ruth Greenway supported me with typing, at the computer and at the various 
stages of the various drafts: I owe them a debt of gratitude.

Study leave and financial assistance were provided by the Government of Jamaica. The subject selected, my consuning interest in and assiduous work on it, evidence my gratitude for this most generous help. My husband deserves the credit for having encouraged me on my course of further study and for introducing me to Andrews University. To him and to my father and two children who patiently and sympathetically waited and prayed and hoped for the "joyful event" I express my warm and sincere appreciation.

The responsibility for any deficiences in the study is mine, but so also is the pleasure of producing something which may be of interest and profit.

Finally, my euphoria at finishing must not hinder my acknowledgment to the One without whose help "no work under the sun" is conceived and accomplished. 
TABLE OF CONTENTS

Page

ACKNOWLEDGENENTS . . . . . . . . . . . . . . . $i i$

LIST OF TABLES . . . . . . . . . . . . . . . . vii vi vi vis

LIST OF FIGURES . . . . . . . . . . . . . . . . . . viii

Chapter

I. INTRODUCTION . . . . . . . . . . . . . . . 1

Statement of the Problem . . . . . . . . . 2

Need for the Stucy................ 3

Purposes of the Study .............. 5

Delimitations of the Study . . . . . . . . . 7

Organization of the Study. . . . . . . . . . 7

II. REVIEW OF THE RELEVANT LITERATURE . . . . . . . . . . 8

Literature on Enrollment Projection Techniques . . . 8

Census Class Projection Method ......... 8

Bell Telephone Method ............. 9

Ratio and Cohort Survival Techniques . . . . . . 10

Housing Projection Method ............ 11

Regression Techniques............. 12

Structural Flow Models . . . . . . . . . 13

Probabilistic Models . . . . . . . . . . . . 17

Literature on Jamaican Population Trends . . . . . 19

Suminary . . . . . . . . . . . . . 22

III. METHODOLOGY . . . . . . . . . . . . . 23

Rationale for District Divisions . . . . . . . . 24

District Categorization on the Basis of ...... 27

the Webster Study . . . . . . . . . . . . . 27

Selection of Districts for Tests... . . . . . . . 3031

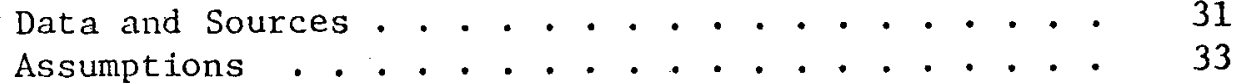

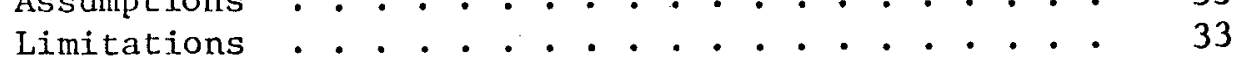

Enrollment Projection Methods . . . . . . . 35

Schedule I... . . . . . . . . . . 36

Schedule II . . . . . . . . . . . . 37

Schedule III................ 39 
Calculation Methods. . . . . . . . . . 40

Summary .................. 40

IV. RESULTS AND ASSESSMENT . . . . . . . . . . : 42

Discreteness of the Districts ......... 42

Tests of the Methodologies........... 43

Performance of Transition Analysis........ 47

Direction of the Projections .......... 50

Correlations and Projections . . . . . . . . 50

Selection of a Method . . . . . . . . . . 54

Projections ............... 57

V. SUMMARY, CONCLUSIONS AND RECOMMENDATIONS . . . . . . 61

Summary .................. . 61

Conclusions . . . . . . . . . . . . . 62

Recommendations ............... 64

APPENDICES

A. Census Class Projection Table .. . . . . . . 68

B. List of Ratio and Regression Methods and

Results of Analysis--The Webster Study (1969) . . . 70

1. List of Ratio and Regression Methods . . . . 71

2. Results of Analysis ........... 73

C. Population Features and Projections--Jamaica. . . . 75

1. Proportional Distributuion of Population by Parish--1921-1970... . . . . . .

2. Parish Population Changes--1960-1970 . . . . .

3. Population Projections--Jamaica--1975-1990 Roberts et al. (1974) .......... 80

4. Population Projections--Jamaica 1970-2020 A.D., Walsh (1970) . . . . . .

D. Description of School Districts . . . . . . .

E. The Delphi Technique ................ 100

F. Computer Printouts of Statistics Used in the Study 
TABLE OF CONTENTS (Continued)

Page

APPENDICES. (Continued)

1. Time Analysis: Eight Year Trend-Seven Districts . . . . . . . . . 105

2. Time Analysis: Six Year Irend--

3. Time Analysis: Six Year and Eight Year Trend--Parish of Hanover . . . . 121

4. Transition Analysis: Eight-Seven Year Trend--Seven Districts . . . . . . . . 124

5. Transition Analysis: Six-Five Year Trend--Seven Districts . . . . . . . . 160

6. Transition Analysis: Eight-Seven Year Trend--Parish of Hanover . . . . . . . . 192

7. Transition Analysis: Six-Five Year Trend-Parish of Hanover .. . . . . . . . . 196

8. Projections to 1981 for Thirteen Districts. Based on September Enrollments and Eight Year Trend . . . . . . . . . . . 208

G. Calculations--The Cohort Survival Method . . . . . 223

BIBLIOGRAPHY . . . . . . . . . . . . . . . 236 


\section{LIST OF TABLES}

Table

1. Comparison of District Projections by the Three Methodologies Based on 6 - 5 Year Trend 1965/1966 - 1970/1971 . . . . . . .

2. Comparison of District Projections by the Three Methodologies Based on $8-7$ Year Trend 1965/1966 - 1972/1973 . . . . . . .

3. Table Showing Pearson r Correlation Co-efficients and Determinations for Time Analysis and Transition Analysis Tests on Which the Results in Table 1 are Based ..........

4. Table Showing Pearson r Correlation Co-efficients and Determinations for the Time Analysis and Transition Analysis Tests on Which Results in Table 2 are Based . . . . . . . . . . . .

5. Comparison of Projections for Parish by the Three Methodologies Based on 6 and 5 Year Trends - 1965/1966 - 1970/1971; also 8 and 7 Year Trends - 1965/1966 1972/1973..................

6. Table Showing Pearson $r$ Correlation Co-efficients and Determinations for Time Analysis and Transition Analysis Tests on Which the Results of Table 5 are Based . . . . . . . . . . . .

7. Ratio of Underestimations to Overestimations by the Three Methodologies: Transition Analysis, Cohort Survival and Time Analysis in the Webster (1969) Study . . . . . . . . . .

8. Comparison of Actual Enrollments and 95 Per Cent Confidence Interval Predictions for The Two Districts by Time Analysis Based on the Two Antecedent Periods . . . . . . . . . .

9. Time Analysis Projections of Pupil Populations to 1981, Parish of Hanover, Jamaica . . . . . . 


\section{LIST OF FIGURES}

\section{Figure}

1. Age Pyramid of Jamaica 1960 and 1970 . . . . . . . . . . .

2. Map of Jamaica . . . . . . . . . . . . .

3. Map of Hanover Showing . Census Districts and School Districts . . . . . . .

4. Jamaica Map of Population Growth (Parish of Hanover) . . . . . . . . . . . .

5. Graph of Data Points, Time Analysis-Kenda1-Upper Rock Spring District . . . . . . .

6. Graph of Data Points, Time Analysis Riverside-Dias District 


\section{CHAPTER I}

\section{INTRODUCTION}

No human undertaking takes place without planning of some type. This planning may be of any gradation along a scale from simple to sophisticated. In the complex societies of today's developed world, planning has become a complicated tool employed by the leadership of most organizations (Brooks 1972). In fact, the more ambitious the enterprise, the more detailed and refined the planning mechanisms employed; and the more precise the planning and evaluation, the more certain of success the results.

Crucial to the planning and evaluation process is an adequate information system (Brooks 1972). The more timely, relevant and exact the information is, then the more valuable it becomes for providing a basis for planning and decision making.

The field of education is no exception. Modern states recognize as their responsibility the education of their citizens. The age group of primary concern, the types of education to be provided, the financial bases for these are matters of governmental policy. Intelligent development or application of these policies depends inevitably on a knowledge of pupil populations. The more precisely these are known in terms of size, categories and locations the more clearly can a government, a state or a district plan the educational 
program. The number of teachers required, their qualifications and specialities, the type and location of facilities, the amount and kinds of amenities and materials to be provided, all are best related directly to this knowledge. From an economic viewpoint, the administration can, on the basis of this knowledge of pupil populations take an inventory of one segment of its human resources and assess its development.

Since planning has a futures orientation, required information on pupil populations goes beyond a description of present numbers. The forecasting of student flows is recognized as a basic and continuing problem for the educational administrator (Greenawalt and Mitche11, 1966; Correa, 1969).

Further, while global forecasts may be informative and valuable, it is at local levels that such information is most pertinent. It is at local levels that the impact of planning mistakes is most acutely felt.

\section{Statement of the Problem}

In view of the above considerations this study investigates the feasibility of making reasonable pupil population forecasts, at local levels, in a developing country, Jamaica. While pupil population projections at nationwide level have been undertaken in Jamaica, little if anything has been done, by way of pupil forecasting at local-or micro levels. It was to this problem that the study addressed itself, focusing on the small rural parish of Hanover, Jamalca. 
Need for the Study

Every September in the recent past, the Janaican educational thents

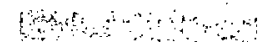

administration has faced the problem of inadequate school accommodation, particularly in the urban areas: Long lines of anxious parents and children wait at primary and all age school doors, while school principals attempt to enlarge already large classes, and the administration makes ad hoc arrangements for emergency classrooms or for redirecting pupils to schools which "can take them."

In the rural areas, a situation of a different kind is hypothesized to exist. Not all children of school age attend school, yet many rural school buildings are under utilized. The question for educational planners, therefore, is: Exactly, where, in relation to the school buildings are the school children, and what will be this relationship in the future?

The vital need for this information is more fully appreciated when the country's socio-economic history is taken into account. This small island, 4,244 square miles in area, supports a population of approximately two million people (Statistical Abstract, 1973, p. 2, Statistical Yearbook of Jamaica, 1974, pp. 1, 117).

The idea of a universal system of public education dates from 1835 when the Negro Education Grant was made to the colonies by the British Government, following the Emancipation Act of 1833.

The financial provisions under the Act ceased in 1845. In the succeeding century, eoucational policy and financial provisions, for a variety of reasons, remained inadequate in terms of utilitarian value and avallability to the populace. The Moyne Report following 
the riots in the West Indies in the late 1930's stated that, "an examination of the working of the educational system reveals serious inadequacies in almost every aspect" (Gordon, 1963, p. 293).

Although significant progress has been made economically and educationally since then, the country is still relatively poor, and educational provision is still inadequate. To illustrate, an average annual growth rate of 10.19 per cent in the Gross Domestic Product is reported as taking place between the years 1953 and 1962. An average of 10.95 per cent was maintained over the years 1963 to 1973, with the average for the five years 1969-1973 being 12.92 per cent (Jamaica; Five Year Independence Plan 1963-1968, p. 12; Statistical Yearbook of Jamaica 1974, p. 965). Still, the majority of the people are poor. Median monthly income in October 1973 was reported as being between $\$ J 10-20$ ( $\$ 11-\$ 22$ U.S.) (Statistical Yearbook, 1974, pp. 675, 700). At the same time 22.4 percent of the labor force was unemployed.

Regarding educational improvements, during the late 1950's and on into the $1960^{\circ}$ 's the government moved to expand educational provision. Foreign aid and loans from Britain, Canada, the United States and the World Bank were secured, enabiing the construction of new primary schools and a new category of schools, the Junior Secondary Schools. Sixty-seven of the latter, with accommodation for 53,000 students now exist. A complementary expansion of high school facilities to receive at least the best of these Junior Secondary School leavers did not take place. To remedy this deficiency, the Government in 1973 decided that all fifteen year olds would be kept on in these schools until age seventeen. 
Concern for the development of the Island's human resources also led to the establishment of Youth Camps and Industrial Training centers, presently under the control of the Ministry of Youth and Community Development. Since 1972 an accelerated program for adult literacy was set up under the Prime Minister's Office. The National Youth Service, introduced in 1973, has placed numbers of young high school graduates to serve in educational institutions.

The provision of facilities for the school age population remains inadequate, however. It was estimated that in 1974 upwards of some 200,000 Jamaican children between the ages of 4 and 17 would not find places in schools (Planning Document--Ministry of Education Jamaica, 1974).

In such a situation there is great need for the educational planners to know as much as possible about the size and location of the country's human resources, particularly those of school age. This knowledge facilitates capital planning and the development of innovative measures and special programs.

\section{Purposes of the Study}

The primary purpose of this study was to test for projection efficiency, at micro levels, the following three enrollment projection methodologies, identified by Webster $(1969,1971)$ as about the most efficient of seventeen projection methodologies which he investigated:

1. Cohort Survival

2. Transition Analysis

3. Time Analysis 
To provide a rationale for the selection of the three methodologies, as appropriate to the Hanover, Jamaica, situation, the literature on enrollment projection methodologies was reviewed extensively. This review provided not only the required rationale but also an overview of projection methodologies which could be of future interest to Jamaican educational planners. As such, it constituted a subsidiary purpose of the study.

In testing the efficiency of the three methodologies it was necessary to simulate as far as possible, the conditions of the Webster (1969) study. First, this involved delineating school "districts" for the parish of Hanover. Since a school district in the American sense, that is, a recognized, discrete administrative unit does not exist in Jamaica, the parish was divided into school districts on the basis of physiographic and economic features which it was felt provided for discreteness. A test for this discreteness was made by comparing the estimated school age population in the districts in two post censal years (1961 and 1971) with the 1961 and 1971 September school district enrollments. Investigating the feasibility of creating such school districts became another subsidiary purpose of the study. Finally, it was intended that, based on the findings regarding efficiency of the methodologies, and the study generally, either projections for future years, or recommendations bearing on the estimation of student flows, would be made. 


\section{Delimitations of the Study}

The fact that the study focuses on a small rural parish in Jamaica must be borne in mind. It cannot be regarded as a typical parish, if such exists.

The bases for the creation of the school districts are necessarily artificial--a fact which must also be borne in mind both in respect of the assumptions regarding discreteness of the districts and in any attempts at practical application.

The census, birth and enrollment data utilized in this study were obtained from official sources but must, as with similar data elsewhere be accepted as less than absolutely accurate.

\section{Organization of the Study}

Chapter I has presented the statement of the problem and the purposes of the study, together with its assumptions and delimitations. It has also commented on the economic-educational situation in Jamaica.

Chapter II reviews the relevant literature.

Chapter III describes the methodology.

Chapter IV analyzes the findings.

Chapter V recapitulates, lists the conclusions and makes recommendations for further work. 
CHAPTER II

REVIEW OF THE RELEVANT LITERATURE

The 1iterature relevant to this study will be reviewed under the following headings:

1. Literature dealing with enrollment projection techniques.

2. Literature dealing with Jamaican population features, including population projections.

\section{Literature on Enrollment Projection Techniques}

The focus of research was on techniques applicable at school district level. The list below is the result of a fairly comprehensive review of the literature. A discussion of the various techniques follows.

1. Census Class Projection Method

2. Bell Telephone Method

3. Ratio and Cohort Survival Techniques

4. Housing Projection Method

5. Regression Techniques

6. Structural Flow Models

7. Probabilistic Models

\section{Census Class Projection Method}

Streve11 (1952) shows that this method depends on a district maintaining a dependable school census from birth to 18 years. By 
posting this data for ten or more years and examining it for internal consistency, migration ratios will be established as well as ratios of the age levels enrolled. From these, projections may be made regarding enrollments in the successive age groups. He points to the necessity of evaluating migration trends (more than 2 per cent to be closely watched) and of identifying limiting factors which may exist in the situation, such as probable economic changes or residential dwelling saturation. He advised that judgment on the broadest array of objective evidence and a thorough inspection of trends in the wider market area must be exercised.

The assumptions of the model are said to be:

1. Private and parochial school attendance will vary with population changes.

2. Educational policy regarding pre-primary attendance will continue unchanged.

3. High school holding power and the like will continue. These assumptions should be tested by field investigation. The Table in Appendix A illustrates the model.

This model would not be applicable in the present study since no such censuses have been taken in the parish. It might be of interest for future work in educational planning.

\section{Be11 Telephone Method}

Holy and Whitehead (1950) describe the Bell Telephone Method as one based on careful studies of total population in small areas, known as house-count sections, within a community. Analyses of births, deaths and migration data are made. The estimates are then translated 
into estimated school enrollments. This method was sald to be particularly useful in determining the distribution of enrollment within the school district.

Like the census class method, this method might be of interest to the educational planner.

\section{Ratio and Cohort Survival Techniques}

This is by far the most common technique described and assessed in the 1iterature (Jaffe, 1969; Larson \& Streve11, 1952; Streve11, 1952; Waski, 1971; Webster, 1969; Zimmer, 1971 among others). Jaffe lists this method as a short tern technique. He shows that it may bear other labels such as Grade Persistence Ratios or be modified using a linear regression method. Residential birth and enrollment data are required, from which are computed ratios of cohort survival or retention from birth to kindergarten, to grade 1 and onwards. The ratios are then either averaged over five years and used, or the ratios used to construct a regression line. These statistics form the basis for predicting future enrollments from existing or estimated data on births and enrollments.

Webster (1969, p. 116), in examining the efficiency of seventeen different kinds of projection methodologies, found the Cohort Survival technique less efficient than his Transition Analysis, a regression technique. However, it proved adequate, according to his criteria, in three out of five of the school district categories in which the various methodologies were tried. Other opinions on the efficiency of this model vary. Joss (1969) did not consider it highly efficient, particularly over long term periods (more than three years). Zimmer (1971) felt, however, that this and the Markov model, 
considered later, offered flexibility in options for improving projections, through incrementing or decrementing probabilities, based on knowledge of systems changes.

It is proposed to test the efficiency of this method in this study.

\section{Housing Projection Method}

Strevel1 (1952) and Jaffe (1969, pp. 39 et seq.) provide significant information on this methodology. An inventory of housing in the community is necessary. Estimates of the average numbers of pupils yielded by each type of housing unit are also necessary. Specially constructed semi-transparent grid maps are used ard layered over the areas under study.

Among the modifying factors to be considered in the use of this technique are:

1. the state of present and future real estate activity in the area;

2. the type of housing and the likely age level and fertility patterns of purchasers; and

3. the occupation probabilities for the particular housing-for example, the question of whether families will move out within five to ten years or remain past child bearing age--and also whether the area's second or third series of occupants will be from middle or lower income groups.

Jaffe (1969) suggests that this method should be used in conjunction with the Cohort Survival method (already described), each a check on the other. 


\section{Regression Techniques}

These may be classified into simple or multiple linear regression models, depending on the number of predictor variables utilized. Webster (1969) examined twelve methods of regression analysis utilizing different combinations of predictor variables and concluded that one--Transition Analysis based on past tendencies of students to advance from one grade to the next--yielded an overall best estimate of enrollment in four out of five types of school districts categorized according to previous population growth. Appendix B1 lists both the ratio and regression models which he investigated and Appendix B2 displays the results.

The following observations taken from this study are worthy of note:

1. Tirear regression is a non-analytic tool in the sense that there is no underlying theory of human behavior which suggests such linear functions (p. 41);

2. due to high intercorrelations among the predictor variables regression equations utilizing more than two predictor variables seemed inapplicable (p. 124); and

3. since Transition Analysis, a regression approach, appeared to supply adequate projections of future school enrollment across all districts; it appeared that one could not go too far wrong in using Transition Analysis (p. 121).

Subsequent to his 1969 study, Webster in 1971 analyzed seven methodologies including Transition Analysis, Cohort Survival and Time Analysis. Time Analysis (past relationship between public school 
enrollment and time) showed considerable accuracy in one of the five district categories--Stratum D. Cohort Survival, the ratio method performed poorly in Stratum B. Transition Analysis and Cohort Survival, in keeping with the results of Webster's previous research, produced significantly more accurate projections than the others. He qualified his conclusions by saying that the results were still tentative and that the study should be replicated. He also felt that in districts with unusual growth characteristics, a local survey that considered a larger number of demographic variables was needed to obtain consistently adequate projections of future school enrollment. Taking account of the above and of Wasik's (1971) conclusion that models of the regression form were likely to provide the most efficient means for singular estimates of enrollment, it is proposed to test the efficiency of two regression methods, Transition Analysis and Time Analysis. This will be in addition to testing the Cohort Survival Method already described.

\section{Structural Flow Models}

Wasik (1971, p. 8) defined structural flows for the purpose of his paper, as models which quantify certain structural relationships among the various factors in the system. He notes that structural models have been widely used to study the flow of students through the system of graduate education in the United States; that the Organization for Economic Co-operation and Development has sponsored the development of models for education utilizing the structural state flow concept; that while the U.S. models were aimed at 
estimating flows through the higher education system, OECD planners have developed models of the primary and secondary educational system which include investigation of how the system relates to the economic and/or social subsystems of a particular country. Correa (1969, pp. 59, 68, 75-77) presents such a model. His premises take account of a situation where enrollment and/or attendance are not compulsory. He therefore presents two methods of prediction--one which considers only the influence of population growth on student flows, and a second which assumes a growth in student population arising from increased per capita income.

His basic equations are built on global figures for new entrants, re-entrants, repeaters from previous school year, graduates, and dropouts such that students at time $t=$

$$
\begin{aligned}
& s_{t}=n_{t}+r_{t}-b_{t}-d_{t}^{1} \\
& s_{t}=g_{t}+a_{t}+r_{t+1}+d_{t}^{2}
\end{aligned}
$$

Where $S=$ number of students at time $t$

$$
\begin{aligned}
& n_{t}=\text { number of new entrants } \\
& r_{t}=\text { number of repeaters from the previous school period } \\
& b_{t}=\text { dropouts before time } \\
& d_{t}=\text { deaths before time } \\
& g_{t}=\text { graduates } \\
& a_{t}=\text { dropouts after time } t \\
& r=\text { repeaters going to the same grade } \\
& t=1=\text { next school period } \\
& d_{t}^{2}=\text { deaths after time } t
\end{aligned}
$$


Projections will be based on ratios of:

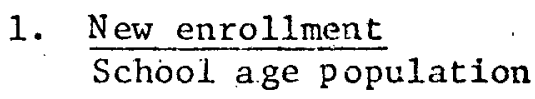

2. $\frac{\text { Repeaters }}{\text { Total inputs }}$

3. Dropouts before pivotal year

4. Deaths before pivotal year

5. Dropouts during and after pivotal year

6. Repeaters passing to next structure

7. Deaths during and after pivotal year

8. $\frac{\text { Graduates }}{\text { Enrollment pivotal year }}$

Equations are provided for estimations at High School and College levels but are similar to the equations cited above, taking into account the relevant ratios.

On the subject of projecting increases in enrollment where less than 100 per cent enter the school system, Correa (1969) assumes a onefactor influence on six-year old entrants, i.e. per capita income. He admits that there may be other influencing factors, however. He introduces the concept of elasticity, $e_{t}$, which is the ratio between increasing proportions of six-year olds entering school and increasing per capita incomes.

The equation is

$$
E_{t}=\frac{\frac{\Delta T_{h 6}}{T_{h 6}}}{\frac{\Delta Y 6}{Y 6}}
$$


Where $\Delta \mathrm{T}_{\mathrm{h} 6}=$ the change from year $\mathrm{h}$ to year $\mathrm{h}+1$ in the value of $t$ (proportions of new entrants) for 6 year olds i.e.

$$
\begin{gathered}
\Delta \mathrm{T}_{\mathrm{h}, 6}=\mathrm{T}_{\mathrm{h}}+1,6-\mathrm{T}_{\mathrm{h}, 6} \\
\mathrm{Y}_{\mathrm{h}}=\text { per capita income in year } \mathrm{h} \text { and } \\
\Delta \mathrm{Y}_{\mathrm{h}}=\text { the change from year } \mathrm{h} \text { to year } \mathrm{h}+1 \text { in the value } \\
\text { of per capita income i.e. } \\
\Delta \mathrm{Y}_{\mathrm{h}}=\mathrm{Y}_{\mathrm{h}}+1-\mathrm{y}_{\mathrm{h}}
\end{gathered}
$$

These elasticity figures for several pairs of years cannot be used uncritically, however. To obtain a figure for projecting new entrants, the average of the last two elasticities is used. The influence of per capita income increase is also calculated into the parameters for graduates, dropouts, repeaters and deaths in elementary school.

Projection of student flows at high school and college levels is also dealt with. Use of the method at college level is subject to modification since results might be unreasonable.

It should be noted that Correa presents a planning model. No comment on its efficiency is made, though reference is made to a computerized application described in a simulation model for educational planning in Puerto Rico.

The model has been described in this detail since it recognizes the potential fluctuations in enrollment arising from economic and other influences in developing countries like Jamaica. where compulsory education has not been enforced. It is not applicable for this study since it would be most applicable to global estimations of enrollment. 


\section{Probabilistic Models}

Markov Chain Models and Monte Carlo simulation models would fall under this heading. They are of particular interest as they offer flexibility for reflecting systems changes which affect enrollment. Mohrenweiser (1969) felt that the Markov model offered additional planning information and permitted simulations of outcomes which might occur from sudden changes in migration and policies. Zimmer (1971) investigated four projection methods against the Minnesota State College System and felt that the Markov chain and the Survival-growth simulation models were flexible in offering options whereby projection accuracy might be improved.

Wasik (1971, p. 10 et seq.) describes the classical Markov process. Subjects within the population of interest are distributed into a set of mutually exclusive "states." These states include various levels of the educational system as well as conditions outside the system. Examples of these states would be Elementary School, Junior High School and other states on through College, then out of school. Parameters of the model are estimated by the obtained transition proportion of the movement of individuals in state $i$ at the time $t$ who will be in the same or different state at time $t+1$. Time units may be defined as quarter, semester or single years.

Since the first application of the Markov Chain Theory to model flow through an educational system by Savage and Brown (1960), several applications have been tried. Studies have been done in Australia (Gani, 1963) to predict enrollment and number of degrees attained in Australian Universities; in Britain (Armitage and Smith, 1967); in the U.S. (Gani, 1965; Wong, 1970) and in Norway (Thonstad, 1967). 
Zabrowski (1967) and Wasik (1971) describe Dynamod II, a modified Markov model. It was used to calculate the numbers of individuals in 140 district population groups over selected periods of time. Separate transition matrices were estimated for each of four sex-race groups across 30 age-educational states with one state to represent deaths. The model equation states that the number of persons in a particular category in the year $t+1$ is equal to the number of persons in that category in year $t$ who remained, plus the number of persons who switch to that category, plus births in the appropriate instances. Zabrowski checked the model against ten year projections developed in the Office of Education and the Bureau of Census and concluded that Dynamod II gave a reasonable fit to these independent external estimates.

The Markov Model requires actual information on the movements of individual students through their educational careers. It therefore will not be used in this study.

Denham (1971) used multivariable techniques on such variables as migration, retention and transfers, producing probable estimates for each: a high, a low and a most likely. A Monte Carlo computer simulation program was written to combine these various estimates into probability distributions of enrollment predictions. It was felt that this method would help the users of enrollment projection information to gain insight into the degree of confidence which the forecaster has in the various estimates.

The method is new and additional tests are required to establish its overall applicability. It will not be used in this study. 
In conclusion, two other areas of observation on enrollment projections should be mentioned. First, Jaffe (1969, p. 19), in commenting on long and short-range projections in small school districts, advises that in districts with school populations below 6,000 , statistically reliable long-range projections are not feasible; that districts of less than 3,000 are definitely too small to make longrange projections; that even short-run projections are of questionable value. In view of this, alternative projections should be made and constantly updated. On the question of long-range projections, he suggests the making of projections of public school enrollments for the state and then working down to county and local school district level. He outlines a number of steps to be taken in developing these estimates but advises against mechanical applications. A knowledge of local conditions is necessary for proper estination. Second, Liu (1966), in considering global estimations of future school enrollments in developing countries, suggests the use of cohort survival and ratio methods, modified by knowledge of past trends, overall population estimates for future years, and new policies.

The question of overall estimates of future population leads to the consideration of the literature on Jamaican population features.

\section{Literature on Jamaican Population Trends}

In a recent study of population movement in Jamaica (Roberts, Powell, Sinclair, Boland \& Hewitt, 1974), pertinent information is provided on Jamaican population trends in general and on population features in the various parishes. Significant features are:

1. continued high fertility rates and low mortality rates; 
2. high external migration rates;

3. the marked rises since 1943 in the population under age 15 as a proportion of the whole due to the factors mentioned above (see Figure 1); and

4. reported improvements in primary school enrollment in the 1970 census.

On the population features of Hanover, the following facts are of interest:

1. as Appendix $\mathrm{Cl}$ on the proportional distribution of population by parish 1921-1970 shows, Hanover slipped from penultimate place in 1921 and 1943 to last place in 1960 and 1970; and

2. according to the tables in Appendix $C 2$ showing parish population changes 1960-1970 Hanover shows a loss in 1970 of 9 per cent over the 1960 figures. In this she ranks eighth among the eleven parishes showing losses.

In the above study, Roberts et al. provide three population projections for the Island up to 1990, based on differing assumptions regarding fertility and migration rates. Details of these three projections broken down into five age groups are provided in Appendix C3.

Projection 1 assumes constant mortality and fertility rates as at 1969-1970 and no external migration. On this basis total population in 1980 would be 2.57 million and in 19903.56 million.

Projection II assumes mortality as at 1969-1970, no migration but appreciable reductions in fertility (crude birth rate down to 20 in 1990). The 1980 population would on these assumptions be 2.338 million and in $1990,2.712$ million. 


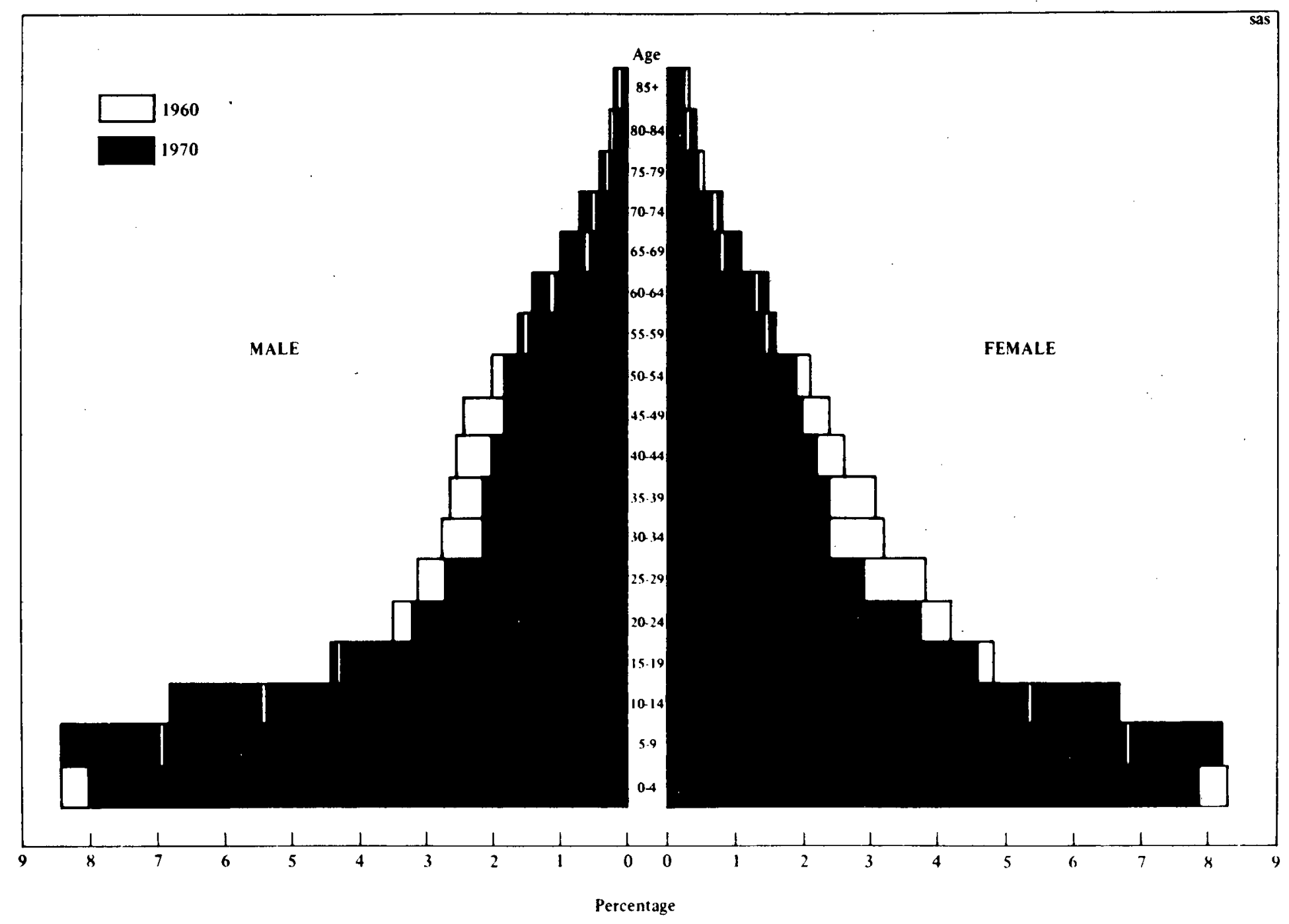

Fig. 1. Age pyramid of Jamaica, 1960 and 1970. 
Projection III assumes the fertility rates of Projection II but sizeable migration rates. The total 1980 population would then be 2.078 million persons and the 1990 population 2.122 million. Walsh (1970) extrapolated Jamaica's population at various rates of growth over a longer period and four fertility rates. These rates were (1) high or 1970 fertility rates, (2) medium or a decline of 1 per cent per annum to 2020 A.D., (3) low fertility rates or 2 per cent per annum reduction, (4) very low, 3 per cent per annum, zero growth at year 2020 A.D. Emigration is not included in his model. It will be noted from Appendix C4 setting out his projections, that his very low at 1990 resembles Robert's very high at 1990 A.D.

\section{Summary}

The statistical methods.surveyed showed that although there is no one best method, certain ratio and regression techniques may provide the best basis for estimating future enrollments in Hanover. The Markov chain system could be useful in providing efficient estimates of differential educational demand.

The information on Jamaican population features and the overall population projections have provided an overview of demographic trends affecting population changes and some overall projections of population. 


\section{CHAPTER III}

\section{METHODOLOGY}

The purpose of this study was to test the efficiency of three enrollment projection methodologies in artifically created school districts in the parish of Hanover, Jamaica.

Since the three methodologies tested were those identified as performing best or adequately in the Webster (1969) study and since his procedures were followed as far as possible, this study may be considered as replicating his study. It is more than a replication however. It is descriptive since it underscores certain special features of the Jamaican educational system. It is exploratory since it examines as a by-product the feasibility of segmenting a parish into school districts by 'natural' process in order to facilitate planning at micro levels. It is predictive since its intent is to provide the means of pupil projection for educational planning.

The topics discussed in this chapter are:

1. Rationale for District Divisions

2. District Categorization on the Basis of Webster's study

3. Selection of Districts for the Tests

4. Data and Sources

5. Assumptions 
6. Limitations

7. Enrollment Projection Methods

8. Calculation Methods

9. Summary

\section{Rationale for District Divisions}

Map 1 (see Figure 2) shows the relationship of the parish of Hanover to the island of Jamaica. Map 2 (see Figure 3) focuses on the parish of Hanover and identifies:

1. the 1970 census subdivisions together with the 1970 distribution of population, and

2. the fourteen "created" school districts listed below.
a. Lucea
b. Green Island
c. Kenda1-Upper Rock Spring
d. Riverside-Dias
e. Askenish
f. Jericho
g. Cascade
h. Sandy Bay
i. Hopewel1
j. Gurney's Mount
k. Mount Peto
1. Flower Hill
m. Chester Castle
n. Friendship-Copse 


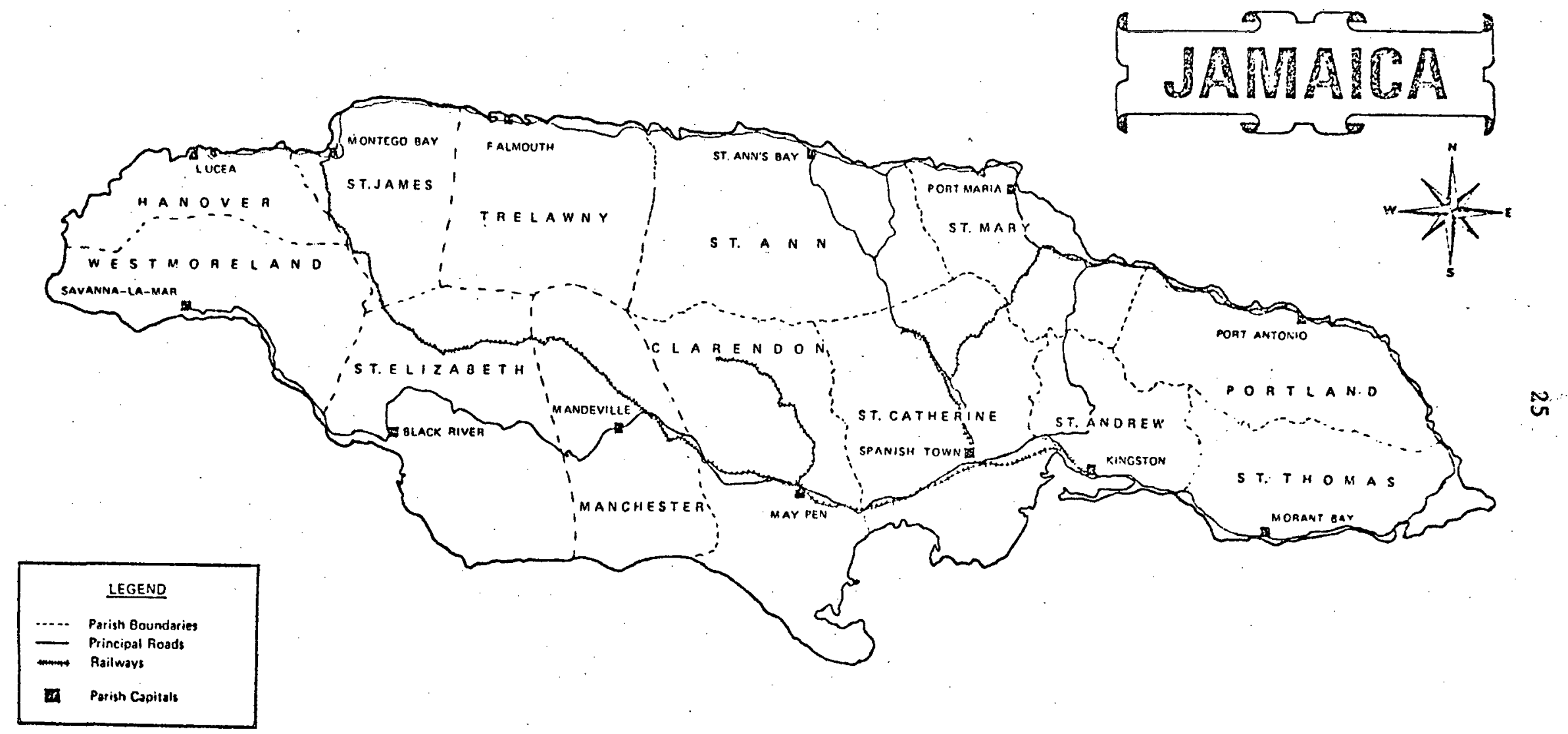

Fig. 2. Map of Jamaica 


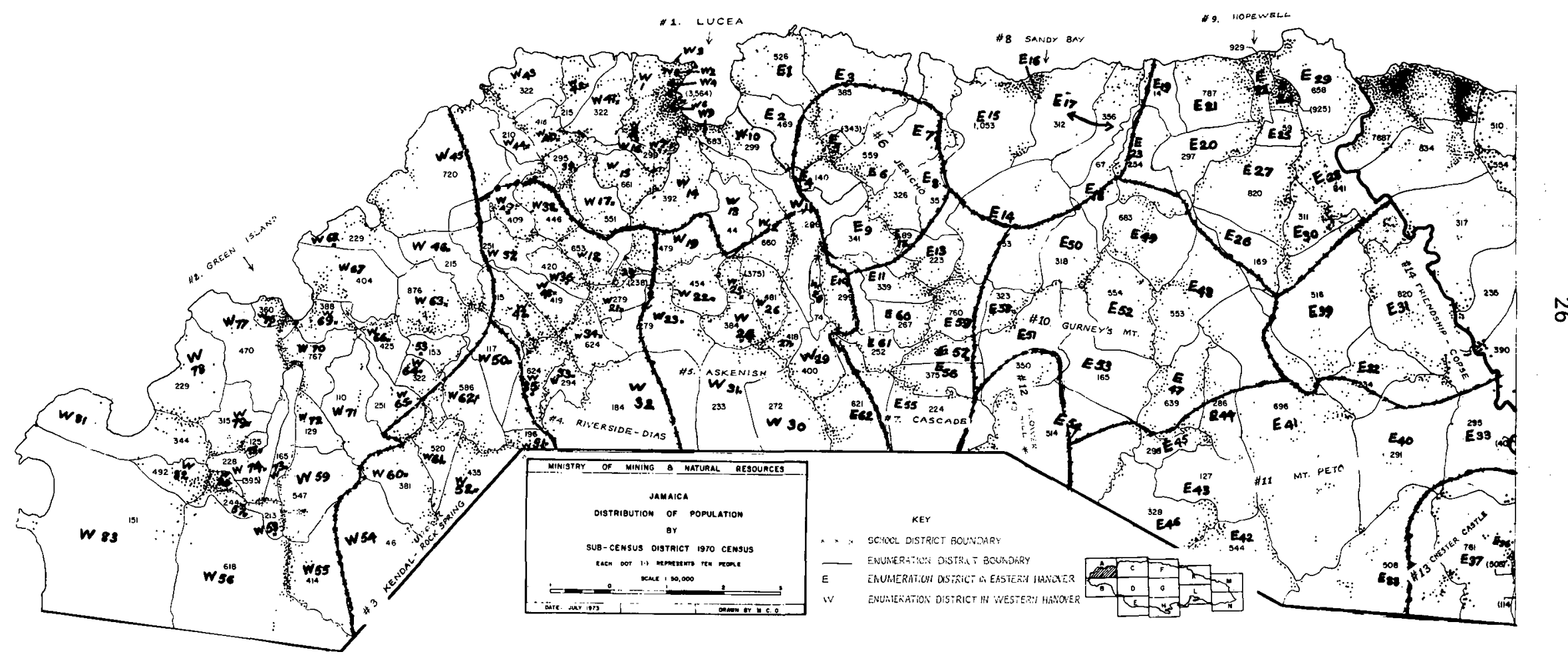

Fig. 3. Map of Hanover Showing Census Districts and School Districts 
A detailed description of the School Districts or zones is provided in Appendix D. This outlines the physiographic, and socioeconomic features of the various districts, shows the assumed school age population in 1961, and 1971, derived from data on the 5-14 age group in the 1960 and 1970 censuses and the September enrollments for 1961,1971 , and 1974.

The natural barriers such as rivers, mountains and uncultivable or uncultivated areas which divide the districts, lend themselves, it is felt, to discreteness. In addition, the recognizable ease of communication within districts lends itself to district homogeneity. These are the primary bases for the particular divisions.

\section{District Categorization on the Basis of the Webster Study}

Webster classified his school districts into five strata "on the basis of certain decision rules involving the population growth variables of the urban place with which the major portion of each district is coterminous" (Webster, 1969, p. 49).

The following were his population growth rules:

Stratum A - increase by more than $100 \%$ in general population and the number of households (these communities called "Exploding Communities");

Stratum B - increase by 50 percent or more in either or both of the classification variables but 100 percent or less in one of these (these communities labeled "Rapidly Growing Communities"); 
Stratum C - increase of 10 percent or more but less than 50 percent in both the number of inhabitants and number of households in the decade (these communities called "O1der but still Growing Communities");

Stratum D - increase of less than 10 percent but equal to or greater than 0 percent in both the number of households and the number of inhabitants or an increase of 10 percent but equal to or greater than 0 percent in one of the classification variables together with an increase of 10 pexcent or more in the other (such communities called "Stable, Well-Established Communities");

Stratum E - decrease in either or both general population and the number of households during the decade (such communities classified as "Declining Conmunities").

The map of population growth (see Figure 4) shows that population change in Jamaica in the decade 1960-1970 has been segmented into seven divisions, i.e.:

$$
\begin{array}{rrr}
-50 & \text { to } & -25 \% \\
-24 & \text { to } & -10 \% \\
-9 & \text { to } & 0 \% \\
0 & \text { to } & 9 \% \\
10 & \text { to } & 24 \% \\
25 & \text { to } & 49 \% \\
50 & \text { and over }
\end{array}
$$

decrease

increase 


\section{J A M A I C A \\ MAP OF POPULATION GROWTH}

Percentage Increase or Decrease In Population Between 1960 and 1970

Sheet A : Western Jamaica

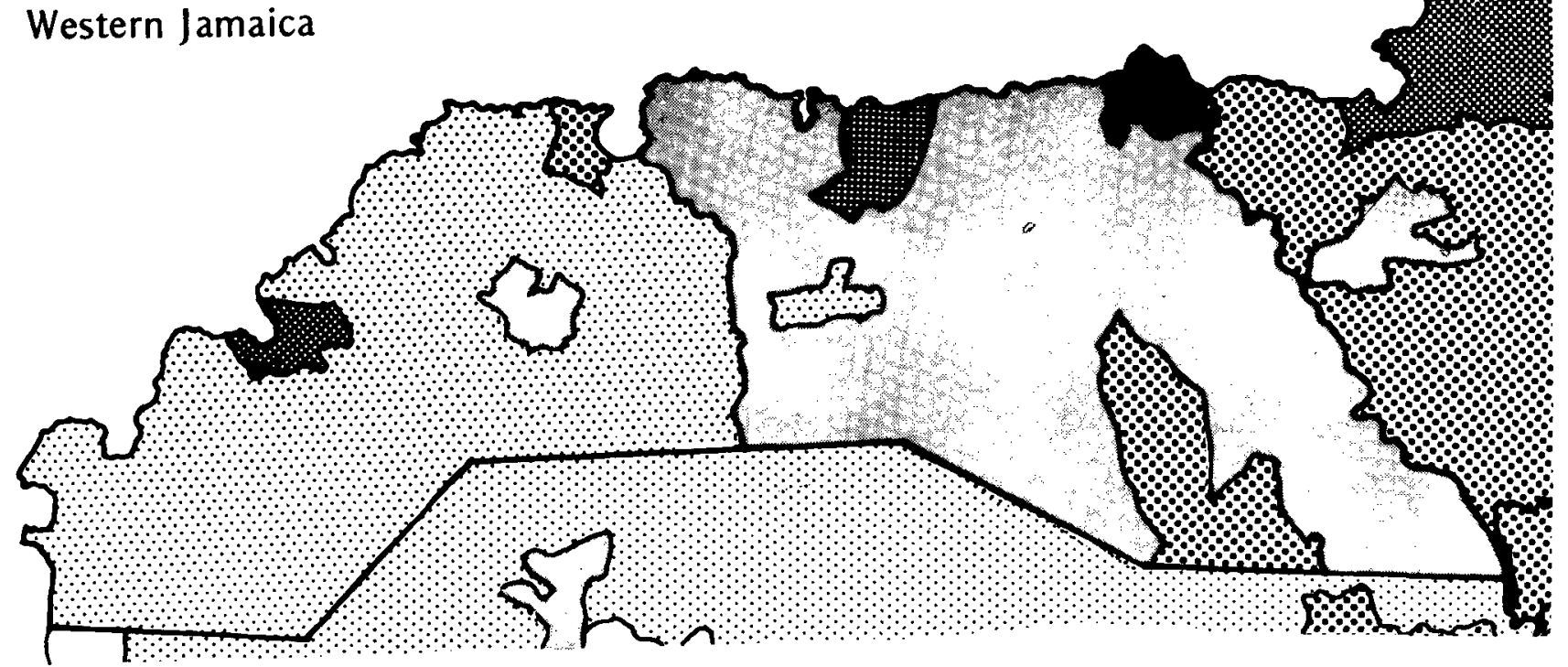

S C A L E $1: 250,000$

\begin{tabular}{l}
$0 \quad 5 \quad 10$ \\
\hline
\end{tabular}

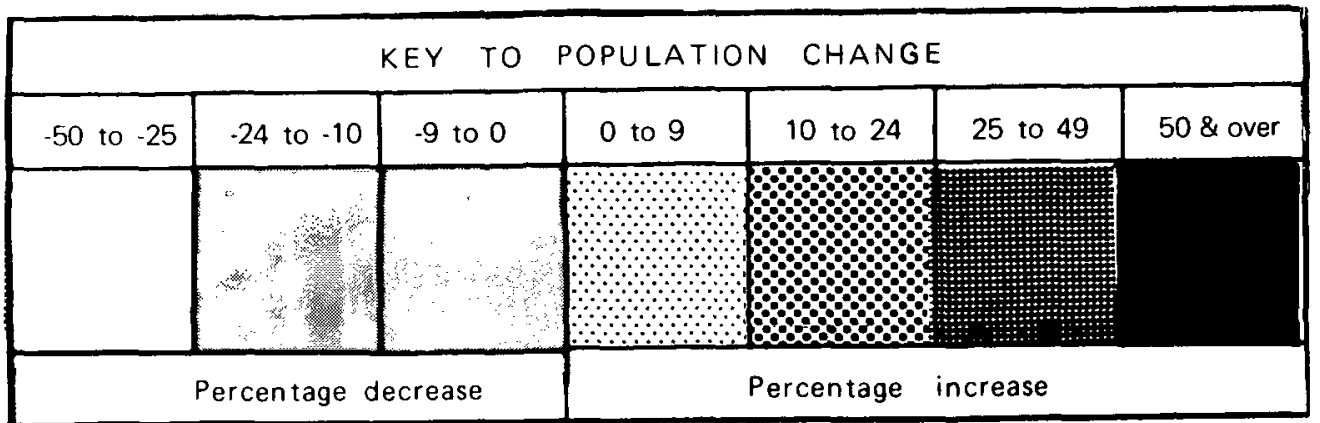

Fig. 4. Jamaica: Map of Population Growth

Parish of Hanover 
According to the map (Figure 4), Hanover shows five rates of population change in the decade but only four on the Webster Classifications since there is no category on the map for growth of over 100 percent. Also, increases of 10-24 percent and 25-49 percent had to be taken together if the Webster study was to be followed. Percentages of household increase were not available for use in this study.

As will readily be seen, Hanover is sharply divided into two categories of population change. The major portion of East Hanover shows a population decline of -9 to 0 percent over the $1960-1970$ decade while the major portion of West Hanover shows a population increase of $0-9$ percent.

\section{Selection of Districts for Tests}

of the fourteen district divisions in Hanover, seven were selected for tests of the methodologies. The seven and their strata assignment here, based on the Webster (1969) study, were as follows:
(cf. Stratum 1 . where population has shown an increase of 50 Webster) percent and above during the decade 1960-70;
District: Hopewell
(cf. Stratum II. where population has shown an increase of 10-24 Webster) or 25-49 percent over the decade 1960-70;
District: Sandy Bay
Stratum III. where population has shown an increase of up to (cf. Stratum $D$,
Webster) 10 percent over the decade 1960-70;
District: Kendal-Upper Rock Spring


Stratum IV. areas where a population decline of $-9-0$ per(cf. Stratum $\mathrm{E}$, Webster) cent took place over the decade 1960-70.

District: Jericho

In addition the following mixed areas were examined:

1. Lucea - where three rates of population change occurred, i.e., a decrease -9 to 0 percent; a small increase $0-10$ percent, and a moderate increase $10-24$ percent.

2. Green Island - which showed increases of 0-9 percent and 25-49 percent.

3. Riverside-Dias which showed a decrease of $-9-0$ percent as well as an increase of 0-9 percent.

\section{Data and Sources}

The data necessary for use in the three methodologies fell into the following categories:

1. residential births for the various districts 1958 through 1967, and

2. enrollment data for students in the 6-15 age group in government owned or operated elementary, junior secondary and secondary schools, since the study is confined to this age group.

To afford comparisons of the numbers of the school-age population and the number of school enrollees, census data and data on enrollments in private schools were also necessary.

Census data and statistics, including tables, maps and books were secured from the Department of Statistics, Jamaica. Data on births for all seventeen registration offices in the parish which 
are related to the districts were personally extracted from the records in the Island Record Office, Spanish Town, Jamaica. These were broken down into quarterly totals but were secured on this basis for only 1961 through 1966 and 1969 through 1974. Yearly totals for 1960 through 1974 were, however, obtained from the Department of Statistics and used for comparison and district allocation. For the missing years 1958 and 1959, totals of births for the parish were estimated by extrapolation. District allocations for these years and for 1960 (for which only the total was available), were effected by using an average of the percentage of total parish births which each district had in the years 1961 and 1962. Likewise, the total of births for 1967 and 1968, obtained from the Department of Statistics, was broken down by district on the basis of similar averages for 1965 and 1966.

The Ministry of Education maintains detailed enrollment and attendance data for the public elementary schools. Data on the following were secured by xeroxing from the Ministry's records:

1. average monthly enrollment and attendance for the primary and all age schools 1966-1967 to 1973-1974 (1969-1970 missing);

2. yearly average enrollment and attendance for the schools for the years 1966-1967 to 1973-1974 (1969-1970 missing);

3. end of year enrollment by grade for the primary, and all age, schools 1965-1966 through 1973-1974; and

4. enrollment by grade 1969-1970 to 1973-1974 for one Junior Secondary School and for only the years 1972-1973 and 1973-1974 for another. Enrollment data for grades 7-9 of the High School were secured for 1971-1972 through 1973-1974 only. Interpolations were, therefore, 
made for missing data.

5. Enrollment data for the private schools was available for the 8-19 age group and for the year 1973-1974 only.

\section{Assumptions}

1. It is assumed that there was no serious under- or overcount in the 1960 and 1970 Censuses in Jamaica.

2. It is assumed that the data on births secured from the Island Record Office are, by and large, accurate.

3. It is assumed that the data and statistics on school enrollment and attendance secured from the Ministry of Education are for the most part reliable.

4. A map similar to Map 2 (see Figure 3) showing the 1960 population clusters was not available. A clustering similar to 1970 was therefore assumed in allocating 1960 census population to divided census/school districts.

\section{Limitations}

\section{Birth Data}

It may safely be assumed that mothers from various locations in the parish have hospital births in the parish capital, Lucea, and register such births there. The total births recorded for the various districts will not be entirely representative of births in those districts, therefore.

2. Allocations of School Age Population

In dividing the various census districts into school districts, population was prorated by using the dots, each of which was said to represent 10 persons. In some cases the number of dots on the map 
bore no relationship to elther the 1960 or 1970 total population for the district nor to the figures appearing on the map. A commonsense approach was consequently adopted. Flowing from the assumption of similar population clustering in 1960 as in 1970, the population allocations to the school districts in 1960 will not be entirely representative of the actual situation. A degree of inaccuracy will also exist in regard to the 1970 allocations.

3. Enrollment data

Available average yearly and September enrollment data were not broken down by grade and did not, therefore, allow for tests of two of the methodologies--Transition Analysis and Cohort Survival on these enrollments. The end-of-school year figures which were broken down in the desired form proved to be very erratic; for example, a peculiar drop exists between grades 5 and 6 in several schools between 1966-1967 and 1967-1968, due apparently to the introduction of a new grade, grade 9, in 1967-1968. This produced irrational allocations between grades 6 through 9 as well.

Finally, and perhaps the most discouraging feature of all, was that some schools snowed the same figures for the individual grades for two and three successive years. The following districts were those most affected:

1. Askenish

2. Cascade

3. Gurneys Mount

4. Hopewel1

5. Mount Peto 
Except for one such district--Hopewell--districts with this drawback were not used in the tests.

In addition, where figures for individual grades were missing or quite irrational, interpolations were made utilizing the least squares equation.

\section{Enrollment Projection Methods}

The enrollment projection methods utilized in this study are described below in terns of type, predictor variables and relationships.

Name

1. Cohort Survival

2. Transition Regression Analysis

3. Time
Analysis

Type

Ratio

.
Regression Time

Births by District; Past district enrollment by grade.

Births by district; Past district enrollment by grade.

\section{Relationship}

Past tendencies of students to advance from one grade to the next shown as a ratio of subsequent grade numbers upon previous ones.

Past tendencies of students to advance from birth to grade one and onwards through each grade.

Past relationship between public school enrollment and time.

Details of the methodologies are shown in Schedules I - III.

The performance of these methods was examined in the seven

districts 1isted on pages 30-31. Goodness-of-fit was then determined, as in the Webster study, by examination of the actual enrollment for predicted year 1973-1974 against the projected enrollment, so that 
Projected district enrollment-Actual enrollment was calculated for Actual Enroliment

each district and the results ranked. The smaller the resulting coefficient, the better the projection. Another measure of efficiency was made by inspecting the methodology which came closest to predicting the actual enrollment figures in the various districts.

\section{Schedule I}

Procedures for Obtaining Projections from the Cohort Survival Ratio Method

1. List actual births by place of residence (district) by year for the period 1958-1959 through 1966-1967 (March to March of each period).

2. List actual enrollment figures by grade 1965-1966 through 1970-1971.

3. Compute average percentage of survivorship between births and Grade 1 enrollment (at $6+-7+$ years) i.e., Grade 1 for five year period as in (1) so that $A(B-1)=$

$\frac{\mathrm{G} 11965 / 66}{\mathrm{~B} 1958-59}+\frac{\mathrm{G} 11966 / 67}{\mathrm{~B} 1959-60}+\frac{\mathrm{G} 11967 / 68}{\mathrm{~B} 1960-61}+\frac{\mathrm{G} 11968 / 69}{\mathrm{~B} 1961-62}+\frac{\mathrm{G} 11969 / 70}{\mathrm{~B} 1962-63}+\frac{\mathrm{Gl} 1970 / 71}{\mathrm{~B} 1963-64}$ N

4. Similarly compute average percentage of survivorship between successive grades so that

$A_{1}-2=\frac{G 21966-67}{G 11965-66}+\frac{G 21967-68}{G 11966-67}+\frac{G 21968-69}{G 11967-68}+\frac{G 21969-70}{G 11968-69}+\frac{G 21970-71}{G 11969-70}$

$\mathrm{N}$

5. Secure, accordingly, the following averages

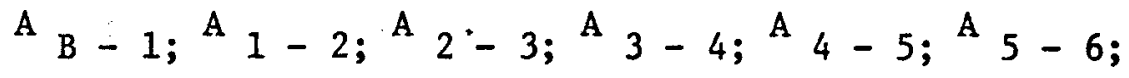

for primary schools and 
${ }^{A} 6-7 ;^{A} 7-8 ;^{A} 8-9$; for All Age Schools and Junior Secondary

School levels.

6. Determine mean births (EB) of students in school in the year of interest (1973-74) i.e. mean births for 1958-69 through 1966-67 for all grades.

7. Calculate enrollment $1973 / 74$ as follows:

1. ${ }^{\mathrm{E}}{ }_{1973-74} \times \mathrm{A}_{\mathrm{B}-1}=\mathrm{G} \mathrm{1}_{1973-74}$

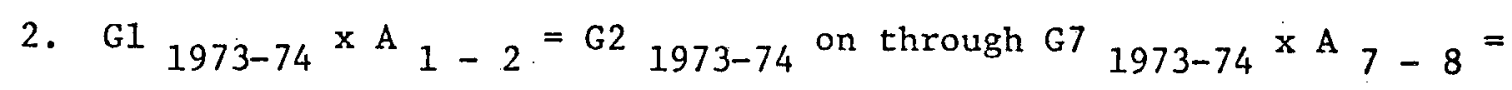
G8 $1973-74$

Sub total for projected total of Grades 1-6 enrollments.

Sub total for projected total of Grades 7-9 enrollments. Make Grand Total.

\section{Schedule II}

Procedures for Obtaining Projections from the Transition Analysis

1. List actual births by place of residence (district), by year, for the period 1958-1959 through 1966-1967.

2. List actual enrollment figures by grade, 1965-1966 through 1970-1971.

3. Utilize the least squares criterion to compute the following nine regression equations with predictor and criterion variables as specified:

\section{Predictor (enrollment)}

a. Births $1958 / 59-1963 / 64$

b. Grade 1 1965/66 - 1969/70

c. Grade 2 1965/66 - 1969/70

d. Grade 3 1965/66 - 1969/70

e. Grade $41965 / 66$ - $1969 / 70$
Criterion (enrollment)

Grade 1 1965/66 - 1970/71

Grade $21966 / 67$ - 1970/71

Grade $3 \quad 1966 / 67$ - 1970/71

Grade $41966 / 67-1970 / 71$

Grade 5 1966/67 - 1970/71 
Predictor (enrollment)

f. Grade 5 1965/66-1969/70

g. Grade 6 1965/66 - 1969/70

h. Grade $71965 / 66$ - 1969/70

i. Grade 8 1965/66 - 1969/70
Criterion (enrollment)

Grade $61966 / 67-1970 / 71$

Grade 7 1966/67 - 1970/71

Grade 8 1966/67 - 1970/71

Grade 9 1966/67 - 1970/71

4. Each of the above is an equation in the form $Y-a+b x$. For example, transition from birth to kindergarten is defined as

$Y=$ projected kindergarten in a given year

$\mathrm{a}=$ the $\mathrm{Y}$ intercept of the line defined by the relationship between births for the years $1958 / 59$ - 1963/64 and Grade 1 enrollment $1966 / 67-1970 / 71$.

$\mathrm{b}=$ the slope of the line defined by the relationship between births for the years 1958/59 - 1963/64 and Grade 1 enrollment for the years $1965 / 66$ - 1970/71.

$x=$ the value of the predictor variable, in this case births during a given period.

5. Determine the mean births for pupils in Grades 1 - 9 in 1973/74; i.e., mean of births for 1961/62 - 1966/67 for Grades 1 - 6 and for $1958 / 59$ - 1960/61 for Grades 7 - 9 .

$(E B=$ mean births by place of residence)

6. Define $\mathrm{T}=\mathrm{a}+\mathrm{b}$ for each of the nine cases in the following manner 1. $\mathrm{T}_{\mathrm{B}-1}(\mathrm{~EB} \operatorname{1973-74})=\mathrm{G} 1$ 1973-74

2. $\mathrm{T}_{1-2}\left(\mathrm{G} 1_{1973-74}\right)=\mathrm{G} 2 \cdot 1973-74$ etc;

so that in $\mathrm{T}_{1-2}\left(\mathrm{Gl}_{1973-74}\right)=\mathrm{G} 2$ 1973-74,

$\mathrm{T}_{1-2}=\mathrm{a}+\mathrm{b}$ where 
$\mathrm{a}=$ the $\mathrm{Y}$ intercept of the line defined by the relationship between Grade 1 enrollment in the years 1965/66 1969/70 and Grade 2 enrollment 1966/67 - 1970/71.

$b=$ the slope of the line defined by the relationship between Grade 1 enrollment in the years 1965/66 1969/70 and Grade 2 enrollment 1966/67 - 1970/71.

GI (1973-74) = Projected Grade 1 enrollment in 1973-74

G2 $(1973-74)=$ Projected Grade 2 enrollment in 1973-74

7. Sum all the estimates to obtain the total projection. (N. B. Webster's steps for Grades 9-11 will not be applied--only one school in the parish has these levels.)

\section{Schedule III}

Procedures Used in Obtaining Projections from Time Analysis I

1. List total district enrollments for the years $1965 / 66$ through $1970 / 71$.

2. Utilize the values $1965 / 66$ through $1970 / 71$ as values of the predictor variable in establishing the required regression equation.

3. Utilizing the least squares criterion compute the equation $\mathrm{Y}=\mathrm{a}+\mathrm{bx}$ to predict enrollment so that

$$
\text { TE }{ }_{1974}=a+b(1974)
$$

where

$\mathrm{TE}=$ Total estimated enrollment

$a=$ the $\mathrm{Y}$ intercept of the line defined by the relationship between time and elementary enrollment for the years 1965/66 through $1970 / 71$.

$b=$ the slope of the line defined by the relationship between time and elementary enrollment for the years 1965/66 through $1970 / 71$ 
1974 = the value of the predictor variable as defined by the year to which. It is desired to project enrollment.

\section{Calculation Methods}

The grade-to-grade ratios required for the Cohort Survival Method were calculated on a desk calculator. The Regression Equations used in the Transition Analysis and the Time Analysis were calculated by the Xerox Sigma 6 computer of Andrews University, use being made of the Simple Linear Regression Program - REG 2 from the Conversational Programs in Statistics in the Computer Library of the University. The Program, described as Simple Linear Regression with residuals, provides information on, among other things:

1. the mean and standard deviation,

2. the correlation and the determination, and

3. the intercept $A$ and the slope $B$.

It also makes 95 percent confidence interval predictions, providing one predicted figure together with upper and lower limits.

\section{Summary}

In this chapter and the related appendices, the fourteen districts delineated for the study were listed and the bases for the divisions described and discussed. The data and their sources were listed and modifications, extrapolations and estimates were described. The assumptions and limitations contingent on the availability, reliability and usefulness of the data were also discussed. The districts selected for testing the methodologies were 1isted, and the methodologies used described in deta11. Departures from the Webster (1969) study 
were also noted.

The flndings arising from these tests are analyzed and dis- cussed in Chapter IV. 


\section{CHAPTER IV}

\section{RESULTS AND ASSESSMENT}

This chapter comments on the apparent discreteness of the fourteen delineated school districts in the parish of Hanover, Jamaica and examines the performance of the three statistical methodologies tested in seven of the fourteen districts.

\section{Discreteness of the Districts}

Reference to Appendix $D$ shows that in ten of the fourteen districts, available September enrollments in 1961 and 1971 were less than the assumed school age populations, derived from data on the 5-14 age group in the 1960 and 1970 censuses respectively.

Two districts, 非 Riverside-Dias and 非 7 Cascade, showed excesses of enrollment over the assumed school age population in 1961 and 1971 respectively. The excess for Riverside (1961), was only 1 percent. For 1971, enrollment was well below the estimated school age population.

The excess at Cascade (1971 only) amounted to 20 percent. Since both enrollment and school age population figures were close in 1961, it appears that this district is drawing pupils from the adjoining ones, particularly Askenish. On-the-spot investigation would show how feasible it is to separate these two districts. Neither Cascade nor Askenish was used in the test. The two other districts showing excesses--Chester 
Castle and Flower H111--1ie on the parish border and are no doubt drawing pupils from the adjoining parishes.

All things considered, the case for discreteness seems to be upheld and, for this reason, the projections and analyses of the study may be related to the districts as partitioned.

\section{Tests of the Methodologies}

The three methodologies tested were:

1. The Cohort Survival Method, the most comonly used method in America which is based on average ratios of survivorship from birth to grade 1 , and from grade 1 to the next succeeding grade--grade 2 and onwards.

2. Transition Analysis also based on the survivorships mentioned above but utilizing the regression equation $\mathrm{Y}=\mathrm{a}+\mathrm{bx}$ to determine a trend line to be used in predictions.

3. Time Analysis--also a regression method which utilizes time and past enrollments as predictors to establish a trend line.

Examination of the results goes beyond the critical assessment of the total enrollment predicted, to evaluation of the behavior of the methodologies in relationship to the particular types of data utilized in the study. The strengths and weaknesses of the three methods are also considered as a basis for decisions on their utilization.

Table 1. presents the results, setting out actual enrollments for the year of interest 1973-1974 and the projections yielded by the three methods, the $W$ (Webster, 1969) coefficient calculated by the following formula, 


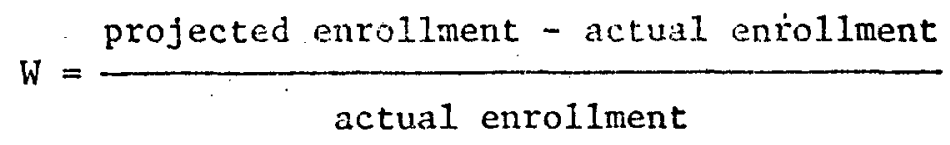

and the rank of the projection among the projections from the three methods.

The projections are ranked from the lowest to the highest $W$ coefficient, the lowest coefficient representing the closest projection.

It will readily be seen that for the seven districts involved in the test and on the basis of one year's projection, Time Analysis performed best of the four methods, in four districts representing Strata II, III, IV; and a district, Riverside, showing mixed moderate population growth. The Cohort Survival Method performed best in two of the districts which, by and large, have more rapid population growth--that is in the Green Island and Lucea districts.

It seemed necessary to check these results against at least a second projection as had been done in the Webster (1969) study. Unavailability of data prior to $1965-1966$ prevented this being done for a year previous to $1973-74$. It was therefore decided to test the methods on a longer trend line--that is, by utilizing data for the period 1965-1966 through 1972-1973, projecting for 1973-1974 and comparing the results with actual enrollment for 1973-1974. Table 2 presents the results of projections on this basis. Time Analysis emerges as best in two of the seven districts, Transition Analysis in two, but Cohort Survival in three.

Overa11, Time Analysis performs best six times, Cohort Survival 
TABLE 1

COMPARISON OF DISTRICT PROJECTIONS BY THE THREE METHODOLOGIES BASED ON 6 - 5 YEAR TREND 1965/1966 - 1970/1971

\begin{tabular}{|c|c|c|c|c|c|c|c|c|c|c|c|}
\hline Stratum* & District & $\begin{array}{l}\text { Actual Enrollment } \\
1973 / 74-\text { Year End }\end{array}$ & $\begin{array}{c}\text { Projections: } \\
\text { Transition } \\
\text { Analysis }\end{array}$ & $\mathrm{W}$ & $\mathrm{R}$ & $\begin{array}{l}\text { Projectic } \\
\text { Cohort } \\
\text { Survival } \\
\end{array}$ & as: & $\mathrm{R}$ & $\begin{array}{c}\text { Projecti } \\
\text { Time } \\
\text { Analysis } \\
\end{array}$ & as: & $\mathrm{R}$ \\
\hline II & Hopewe11 & 1979 & 1656 & -.1632 & 3 & 1868 & -.0560 & 2 & 2003 & +.0121 & 1 \\
\hline III & Sandy Bay & 786 & 622 & -.2086 & 3 & 856 & +.0890 & 2 & 798 & +.0153 & 1 \\
\hline IV & $\begin{array}{l}\text { Kenda1 - Upper } \\
\text { Rock Spring }\end{array}$ & 764 & 778 & +.0183 & 2 & 791 & +.0353 & 3 & 767 & +.0039 & 1 \\
\hline V & Jericho & 687 & 690 & +.0058 & 1 & 723 & +.0524 & 2 & 733 & +.0670 & 3 \\
\hline Mixed & Lucea & 2177 & 1540 & -.2926 & 3 & 1758 & -.1925 & 1 & 1592 & -.2687 & 2 \\
\hline Mixed & Green Island & 2866 & 2505 & -.1259 & 2 & 2768 & -.0341 & 1 & 3248 & +.1333 & 3 \\
\hline Mixed & $\begin{array}{l}\text { Riverside- } \\
\text { Dias }\end{array}$ & 1699 & 1808 & +.0642 & 2 & 1940 & +.1418 & 3 & 1700 & .0005 & 1 \\
\hline
\end{tabular}

$W=$ Webster (1969) co-efficient $=\frac{\text { projected enrollment }- \text { actual enrollment }}{\text { actual enrollment }}$

$R=$ Rank among the three methods

*Criteria for strata based on Webster (1969) study. 
TABLE 2

COMPARISON OF DISTRICT PROJECTIONS BY THE THREE METHODOLOGIES BASED ON 8 - 7 YEAR TREND 1965/1966 - 1972/1973

\begin{tabular}{|c|c|c|c|c|c|c|c|c|c|c|c|}
\hline Stratum* & District & $\begin{array}{l}\text { Actual Enrollment } \\
1973 / 74 \text { Year End }\end{array}$ & $\begin{array}{l}\text { Projections: } \\
\text { Transition } \\
\text { Analysis }\end{array}$ & $\mathrm{W}$ & $\mathrm{R}$ & $\begin{array}{l}\text { Projectio } \\
\text { Cohort } \\
\text { Survival } \\
\end{array}$ & $\overline{\text { ns: }}$ & $\mathrm{R}$ & $\begin{array}{l}\text { Projectio } \\
\text { Time } \\
\text { Analysis } \\
\end{array}$ & Is: & $\underline{\mathbf{R}}$ \\
\hline II & Hopewell & 1979 & 1714 & -.1339 & 3 & 1976 & -.0015 & 1 & 1932 & -.0237 & 2 \\
\hline III & Sandy Bay. & 786 & 666 & -.1527 & 3 & 853 & +.0852 & 2 & 783 & -.0038 & 1 \\
\hline IV & $\begin{array}{l}\text { Kendal - Upper } \\
\text { Rock Spring }\end{array}$ & 764 & 754 & -.0131 & 1 & 792 & +.0366 & 2 & 735 & -.0380 & 3 \\
\hline V & Jericho & 687 & 686 & -.0015 & 1 & 826 & +.2023 & 3 & 724 & +.0539 & 2 \\
\hline Mixed & Lucea & 2177 & 1603 & -.2637 & 3 & 1961 & -.0992 & 1 & 1898 & -.1282 & 2 \\
\hline Mixed & Green Island & 2866 & 2547 & -.1113 & 3 & 3051 & +.0645 & 1 & 3136 & +.0942 & 2 \\
\hline Mixed & Riverside & 1699 & 1780 & +.0476 & 2 & 1887 & +.1107 & 3 & 1687 & -.0071 & 1 \\
\hline
\end{tabular}

$\mathrm{W}=$ Webster $(1969)$ co-efficient $=$ projected enrollment - actual enrollment actual enrollment

$\mathrm{R}=$ Rank among the three methods

*Criteria for strata based on Webster (1969) study. 
five times. If efficiency is assessed on the basis of number of projections falling below 10 percent of over- or under-estimation of the actual figures, it will be seen that Time Analysis and Cohort Survival ranked almost equally with eleven and ten respectively out of fourteen projections within the 10 percent limit in Tables 1 and 2 taken together. Transition Analysis ranked third with only six out of fourteen projections falling below the 10 percent limit.

On the basis of Table 1, Time Analysis would be assessed as performing better than the other two methods. On the basis of Tables $I$ and 2 taken together, a clear differentiation is difficult to make. It may be observed, however, that Time Analysis has the slight edge over the Cohort Survival Method while Transition Analysis is not as efficient.

\section{Performance of Transition Analysis}

The question arises as to why Transition Analysis performed so poorly in contrast to its performance in the Webster (1969) study. It was hypothesized that the regression equation was especially sensitive to variability in the erratic grade-to-grade figures; that correlations would be lower in Transition Analysis than for Time Analysis. Details of the correlations and determinations are shown in Tables 3 and 4. These contain:

1. the correlations and determinations for Time Analysis and for the nine equations used for each of the districts' Transitional Analyses; and

2. the percentage of events in transition analysis with correlations $\geq .50$ 
TABLE 3

TABLE SHOWING PEARSON $\mathrm{r}$ CORRELATION CO-EFFICIENTS AND DETERMINATIONS FOR TIME ANALYSIS AND TRANSITION ANALYSIS TESTS ON WHICH THE RESULTS IN TABLE 1 ARE BASED

\begin{tabular}{|c|c|c|c|c|c|c|c|c|c|c|c|c|c|}
\hline Stratum & District & & $\begin{array}{l}\text { Time } \\
\text { Inalysis }\end{array}$ & $B-1$ & $1-2$ & $2-3$ & $\begin{array}{l}\text { Trans } \\
3-4\end{array}$ & $\underset{4-5}{\operatorname{ition} \mathrm{An}}$ & $\begin{array}{r}\text { alysis } \\
5-6\end{array}$ & $6-7$ & $7-8$ & $8-9$ & $\begin{array}{c}\% \text { of Events in } \\
\text { Transition } \\
\text { Analysis with } \\
\text { Correlation } \geqslant .50\end{array}$ \\
\hline II & Hopewe11 & $\begin{array}{l}\mathrm{C} \\
\mathrm{D}\end{array}$ & $\begin{array}{l}.7858 \\
.6176\end{array}$ & $\begin{array}{r}-.1699 \\
.0287\end{array}$ & $\begin{array}{l}.9224 \\
.8509\end{array}$ & $\begin{array}{l}.9539 \\
.9099\end{array}$ & $\begin{array}{l}.3938 \\
.1550\end{array}$ & $\begin{array}{l}.5496 \\
.3021\end{array}$ & $\begin{array}{r}-.8576 \\
.7355\end{array}$ & $\begin{array}{r}-.4924 \\
.2442\end{array}$ & $\begin{array}{r}-.2054 \\
.0422\end{array}$ & $\begin{array}{l}.4666 \\
.2177\end{array}$ & 44 \\
\hline III & Sandy Bay & $\begin{array}{l}\mathrm{C} \\
\mathrm{D}\end{array}$ & $\begin{array}{l}.8975 \\
.8054\end{array}$ & $\begin{array}{r}-.5204 \\
.2708\end{array}$ & $\begin{array}{l}.8241 \\
.6791\end{array}$ & $\begin{array}{l}.8621 \\
.7433\end{array}$ & $\begin{array}{l}.0022 \\
.0000\end{array}$ & $\begin{array}{l}.3627 \\
.1315\end{array}$ & $\begin{array}{r}-.2233 \\
.0499\end{array}$ & $\begin{array}{r}-.2243 \\
.0530\end{array}$ & $\begin{array}{l}.1084 \\
.0118\end{array}$ & $\begin{array}{l}.7712 \\
.5948\end{array}$ & 44 \\
\hline IV & $\begin{array}{l}\text { Kendal-Upper } \\
\text { Rock Spring }\end{array}$ & $\begin{array}{l}\text { C } \\
\text { D }\end{array}$ & $\begin{array}{r}-.0098 \\
.0000\end{array}$ & $\begin{array}{l}.1287 \\
.0166\end{array}$ & $\begin{array}{l}.7943 \\
.6310\end{array}$ & $\begin{array}{l}.9032 \\
.8157\end{array}$ & $\begin{array}{l}.9233 \\
.8524\end{array}$ & $\begin{array}{l}.7569 \\
.5730\end{array}$ & $\begin{array}{r}-.5071 \\
.2572\end{array}$ & $\begin{array}{l}.8515 \\
.7251\end{array}$ & $\begin{array}{l}.0853 \\
.0073\end{array}$ & $\begin{array}{l}.4689 \\
.2198\end{array}$ & 67 \\
\hline V & Jericho & $\begin{array}{l}\text { C } \\
\text { D }\end{array}$ & $\begin{array}{l}.5862 \\
.3436\end{array}$ & $\begin{array}{l}.3704 \\
.1372\end{array}$ & $\begin{array}{l}.2657 \\
.0706\end{array}$ & $\begin{array}{l}.9248 \\
.8553\end{array}$ & $\begin{array}{l}.6341 \\
.4021\end{array}$ & $\begin{array}{l}.1871 \\
.0350\end{array}$ & $\begin{array}{l}.2612 \\
.0682\end{array}$ & $\begin{array}{r}-.7851 \\
.6164\end{array}$ & $\begin{array}{l}.0904 \\
.0082\end{array}$ & $\begin{array}{r}-.2199 \\
.0484\end{array}$ & 33 \\
\hline Mixed & Lucea & $\begin{array}{l}\text { C } \\
\text { D }\end{array}$ & $\begin{array}{l}.5639 \\
.3180\end{array}$ & $\begin{array}{r}-.0841 \\
.0071\end{array}$ & $\begin{array}{l}.1412 \\
.0199\end{array}$ & $\begin{array}{l}.0586 \\
.0034\end{array}$ & $\begin{array}{l}.1695 \\
.0287\end{array}$ & $\begin{array}{l}.3736 \\
.1396\end{array}$ & $\begin{array}{r}-.2126 \\
.0452\end{array}$ & $\begin{array}{l}.3939 \\
.1552\end{array}$ & $\begin{array}{l}.9033 \\
.8160\end{array}$ & $\begin{array}{r}-.0200 \\
.0004\end{array}$ & 11 \\
\hline Mixed & $\begin{array}{l}\text { Green } \\
\text { Island }\end{array}$ & $\begin{array}{l}\mathrm{C} \\
\mathrm{D}\end{array}$ & $\begin{array}{l}.9695 \\
.9399\end{array}$ & $\begin{array}{r}-.1450 \\
.0210\end{array}$ & $\begin{array}{l}.6457 \\
.4170\end{array}$ & $\begin{array}{l}.8191 \\
.6710\end{array}$ & $\begin{array}{l}.9903 \\
.9806\end{array}$ & $\begin{array}{l}.2490 \\
.0620\end{array}$ & $\begin{array}{r}-.4109 \\
.1689\end{array}$ & $\begin{array}{r}-.5377 \\
.2891\end{array}$ & $\begin{array}{l}.8072 \\
.6516\end{array}$ & $\begin{array}{l}.7927 \\
.6284\end{array}$ & 67 \\
\hline Mixed & Riverside & $\begin{array}{l}C \\
D\end{array}$ & $\begin{array}{r}-.3038 \\
.0923\end{array}$ & $\begin{array}{l}.8413 \\
.7077\end{array}$ & $\begin{array}{r}-.2946 \\
.0868\end{array}$ & $\begin{array}{r}-.6539 \\
.4276\end{array}$ & $\begin{array}{l}.6349 \\
.3905\end{array}$ & $\begin{array}{r}-.2080 \\
.0433\end{array}$ & $\begin{array}{r}-.2464 \\
.0607\end{array}$ & $\begin{array}{r}-.8563 \\
.7333\end{array}$ & $\begin{array}{r}-.2708 \\
.0733\end{array}$ & $\begin{array}{l}.1459 \\
.0213\end{array}$ & 44 \\
\hline
\end{tabular}


TABLE 4

TABLE SHOWING PEARSON $\mathrm{r}$ CORRELATION CO-EFFICIENTS AND DETERMINATIONS

FOR THE TIME ANALYSIS AND TRANSITION ANALYSIS TESTS ON WHICH RESULTS IN TABLE 2 ARE BASED

\begin{tabular}{|c|c|c|c|c|c|c|c|c|c|c|c|c|c|c|}
\hline Stratum & District & & $\begin{array}{c}\text { Time } \\
\text { Analysis }\end{array}$ & $B-1$ & $1-2$ & $2-3$ & $\begin{array}{l}\text { Trans } \\
3-4\end{array}$ & $\underset{4-5}{\text { ition } A n}$ & $\begin{array}{r}\text { alysis } \\
5-6\end{array}$ & $6-7$ & $7-8$ & $8-9$ & $\begin{array}{c}\% \text { of Even } \\
\text { Transit } \\
\text { Analysis } \\
\text { Correlation }\end{array}$ & $\begin{array}{l}\text { its in } \\
\text { =ion } \\
\text { with } \\
=.50\end{array}$ \\
\hline I & Hopewe 11 & $\begin{array}{l}\mathrm{C} \\
\mathrm{D}\end{array}$ & $\begin{array}{l}.8493 \\
.7214\end{array}$ & $\begin{array}{r}-.1995 \\
.0398\end{array}$ & $\begin{array}{l}.7330 \\
.5373\end{array}$ & $\begin{array}{l}.9316 \\
.8678\end{array}$ & $\begin{array}{l}.5250 \\
.2757\end{array}$ & $\begin{array}{l}.5483 \\
.3006\end{array}$ & $\begin{array}{r}-.7580 \\
.5745\end{array}$ & $\begin{array}{r}-.1053 \\
.0111\end{array}$ & $\begin{array}{r}-.0224 \\
.0005\end{array}$ & $\begin{array}{l}.4276 \\
.1829\end{array}$ & 56 & \\
\hline II & Sandy Bay & $\begin{array}{l}\mathrm{C} \\
\mathrm{D}\end{array}$ & $\begin{array}{l}.9437 \\
.8905\end{array}$ & $\begin{array}{l}.0271 \\
.0007\end{array}$ & $\begin{array}{l}.8608 \\
.7409\end{array}$ & $\begin{array}{l}.9571 \\
.9161\end{array}$ & $\begin{array}{l}.4177 \\
.1745\end{array}$ & $\begin{array}{l}.4478 \\
.2006\end{array}$ & $\begin{array}{r}-.1272 \\
.0162\end{array}$ & $\begin{array}{r}-.5204 \\
.2708\end{array}$ & $\begin{array}{r}-.2017 \\
.0407\end{array}$ & $\begin{array}{l}.5923 \\
.3508\end{array}$ & 22 & \\
\hline III & $\begin{array}{l}\text { Kendal-Upper } \\
\text { Rock Spring }\end{array}$ & $\begin{array}{l}\mathrm{C} \\
\mathrm{D}\end{array}$ & $\begin{array}{r}-.2221 \\
.0493\end{array}$ & $\begin{array}{l}.3914 \\
.1532\end{array}$ & $\begin{array}{l}.7350 \\
.5403\end{array}$ & $\begin{array}{l}.8182 \\
.6694\end{array}$ & $\begin{array}{l}.8913 \\
.7944\end{array}$ & $\begin{array}{l}.7191 \\
.5171\end{array}$ & $\begin{array}{r}-.3810 \\
.1452\end{array}$ & $\begin{array}{l}.8646 \\
.7475\end{array}$ & $\begin{array}{l}.3466 \\
.1202\end{array}$ & $\begin{array}{l}.6007 \\
.3609\end{array}$ & 56 & \\
\hline IV & Jericho & $\begin{array}{l}\text { C } \\
\text { D }\end{array}$ & $\begin{array}{l}.6953 \\
.4834\end{array}$ & $\begin{array}{r}-.0671 \\
.0045\end{array}$ & $\begin{array}{l}.5240 \\
.2746\end{array}$ & $\begin{array}{l}.7159 \\
.5124\end{array}$ & $\begin{array}{l}.4907 \\
.2408\end{array}$ & $\begin{array}{r}-.0078 \\
.0001\end{array}$ & $\begin{array}{l}.4825 \\
.2328\end{array}$ & $\begin{array}{r}-.6673 \\
. .4452\end{array}$ & $\begin{array}{r}-.1795 \\
.0322\end{array}$ & $\begin{array}{l}.1783 \\
.0318\end{array}$ & 33 & 8 \\
\hline Mixed & Lucea & $\begin{array}{l}\mathrm{C} \\
\mathrm{D}\end{array}$ & $\begin{array}{l}.7435 \\
.5528\end{array}$ & $\begin{array}{r}-.6435 \\
.4141\end{array}$ & $\begin{array}{l}.2742 \\
.0752\end{array}$ & $\begin{array}{l}.2377 \\
.0565\end{array}$ & $\begin{array}{r}.2950 \\
-.0870\end{array}$ & $\begin{array}{l}.6687 \\
.4471\end{array}$ & $\begin{array}{l}-.2127 \\
-.0452\end{array}$ & $\begin{array}{r}.2653 \\
-.0704\end{array}$ & $\begin{array}{l}.3447 \\
.1188\end{array}$ & $\begin{array}{r}-.7089 \\
.5025\end{array}$ & 33 & \\
\hline Mixed & $\begin{array}{l}\text { Green } \\
\text { Island }\end{array}$ & $\begin{array}{l}\mathrm{C} \\
\mathrm{D}\end{array}$ & $\begin{array}{l}.9763 \\
.9531\end{array}$ & $\begin{array}{l}-.1900 \\
-.0361\end{array}$ & $\begin{array}{l}.6344 \\
.4025\end{array}$ & $\begin{array}{l}.8941 \\
.7994\end{array}$ & $\begin{array}{l}.9676 \\
.9362\end{array}$ & $\begin{array}{l}.8001 \\
.6402\end{array}$ & $\begin{array}{l}.5015 \\
.2515\end{array}$ & $\begin{array}{r}-.5484 \\
.3007\end{array}$ & $\begin{array}{l}.8383 \\
.7028\end{array}$ & $\begin{array}{l}.8904 \\
.7928\end{array}$ & 89 & \\
\hline Mixed & Riverside & $\begin{array}{l}\text { C } \\
\text { D }\end{array}$ & $\begin{array}{r}-.4275 \\
.1828\end{array}$ & $\begin{array}{l}.7616 \\
.5801\end{array}$ & $\begin{array}{r}-.0393 \\
.0015\end{array}$ & $\begin{array}{r}-.5322 \\
.2832\end{array}$ & $\begin{array}{l}.5157 \\
.2659\end{array}$ & $\begin{array}{r}-.1608 \\
.0259\end{array}$ & $\begin{array}{l}-.3087 \\
-.0953\end{array}$ & $\begin{array}{r}-.6892 \\
.4750\end{array}$ & $\begin{array}{l}-.0491 \\
-.0024\end{array}$ & $\begin{array}{l}.4674 \\
.2185\end{array}$ & 44 & \\
\hline
\end{tabular}


It was also questioned whether the smallness of the district figures in addition their greater variations contributed to the poor performance of this method. To assess the performance of the three methodologies on larger figures, the methods were tested on the parish totals. Table 5 sets out the results. There, Transition Analysis performed best of the three methodologies, underestimating the enrollment by small percentages. Cohort Survival and Time Analysis performed less efficiently and at about equal levels. Table 6 shows that here higher correlations were achieved by both regression methods.

\section{Direction of the Projections}

An interesting observation was made on the direction of the projections. Transition Analysis underestimated the enrollment in the year of interest more (twelve out of sixteen times) than did either Cohort Survival or Time Analysis (six times respectively). This prompted a comparison with the Webster study. Table 7 summarizes the rate of the underestimations in the Webster study. It will be seen that, over a11, Transition Analysis tends to underestimate while Time Analysis tends to overestimate. The underestimations were more pronounced at secondary levels, the overestimations at primary level.

\section{Correlations and Projections}

It will be observed that, overall, Time Analysis achieved higher correlations than Transition Analysis; that in Transition Analysis birth to Grade 1 correlations were low in all districts. Table 3 shows that in only one district did correlations of .50 or more eventuate more than 50 percent of the time. Table 2 shows that this increased to three districts. 
TABLE 5

COMPARISON OF PROJECTIONS FOR PARISH BY THE THREE METHODOLOGIES

BASED ON 6 AND 5 YEAR TRENDS - 1965/1966 - 1970/1971

\begin{tabular}{|c|c|c|c|c|c|c|c|c|c|c|}
\hline Parish & $\begin{array}{c}\text { Actual } \\
\text { Enroliment } \\
1973-1974 \\
\end{array}$ & $\begin{array}{l}\text { Projections: } \\
\text { Transition } \\
\text { Analysis }\end{array}$ & $\mathrm{W}$ & $\mathrm{R}$ & $\begin{array}{l}\text { Projectio } \\
\text { Cohort } \\
\text { Survival } \\
\end{array}$ & $\mathrm{W}$ & $\mathrm{R}$ & $\begin{array}{l}\text { Projectic } \\
\text { Time } \\
\text { Analysis }\end{array}$ & W & $\mathrm{R}$ \\
\hline Hanover & 14,051 & 13,520 & -.0378 & 1 & 14,688 & -.0439 & 2 & 14,775 & +.0515 & 3 \\
\hline
\end{tabular}

COMPARISON OF PROJECTIONS FOR PARISH BY THE THREE METHODOLOGIES BASED ON 8 AND 7 YEAR TRENDS - 1965/1966 - 1972/1973

\begin{tabular}{lclllllllll}
\hline & $\begin{array}{c}\text { Actual } \\
\text { Enrollment } \\
1973-1974\end{array}$ & $\begin{array}{l}\text { Projections: } \\
\text { Transition } \\
\text { Analysis }\end{array}$ & W & R & $\begin{array}{l}\text { Cohort } \\
\text { Survival }\end{array}$ & W & R & $\begin{array}{l}\text { Pime Analysis } \\
\text { Parish }\end{array}$ \\
\hline Hanover & 14,051 & 13,828 & -.0159 & 1 & 14,790 & +.0526 & 3 & 14,475 & +.0302 & 2 \\
\hline
\end{tabular}

$\mathrm{W}=$ Webster's (1969) Co-efficient $=$ projected enrollment - actual enrollment actual enroliment

$\mathrm{R}=$ Rank among the methods 
TABLE 6

TABLE SHOWING PEARSON $\mathrm{r}$ CORRELATION CO-EFFICIENTS AND DETERMINATIONS FOR TIME ANALYSIS AND TRANSITION ANALYSIS TESTS ON WHICH THE RESULTS OF TABLE 5 ARE BASED

\begin{tabular}{|c|c|c|c|c|c|c|c|c|c|c|c|}
\hline Hanover & $\begin{array}{c}\text { Time } \\
\text { Analysis }\end{array}$ & $B-1$ & $1-2$ & $2-3$ & $3-4$ & $4-5$ & $5-6$ & $6-7$ & $7-8$ & $8-9$ & $\begin{array}{c}\% \text { of } \\
\text { Correlation } \\
\geq .50\end{array}$ \\
\hline $6-5$ Years & $\begin{array}{l}.8620 \\
.7430\end{array}$ & $\begin{array}{r}-.5639 \\
.3180\end{array}$ & $\begin{array}{r}.9572 \\
.9161\end{array}$ & $\begin{array}{l}.9943 \\
.9885\end{array}$ & $\begin{array}{l}.7830 \\
.6131\end{array}$ & $\begin{array}{l}.2759 \\
.0761\end{array}$ & $\begin{array}{r}-.8563 \\
.7333\end{array}$ & $\begin{array}{r}-.6453 \\
.4160\end{array}$ & $\begin{array}{r}-.4346 \\
.1888\end{array}$ & $\begin{array}{l}.7922 \\
.6276\end{array}$ & .77 \\
\hline 8-7 Years & $\begin{array}{l}.8990 \\
.8081\end{array}$ & $\begin{array}{r}-.5985 \\
.3582\end{array}$ & $\begin{array}{l}.9411 \\
.8857\end{array}$ & $\begin{array}{l}.9819 \\
.9641\end{array}$ & $\begin{array}{l}.9493 \\
.9012\end{array}$ & $\begin{array}{r}.8935 \\
.7983\end{array}$ & $\begin{array}{r}-.3927 \\
.1542\end{array}$ & $\begin{array}{r}-.5684 \\
.3231\end{array}$ & $\begin{array}{l}.1690 \\
.0285\end{array}$ & $\begin{array}{l}.6760 \\
.4569\end{array}$ & 77 \\
\hline
\end{tabular}


TABLE 7

RATIO OF UNDERESTIMATIONS TO OVERESTIMATIONS BY THE THREE METHODOLOGIES: TRANSITION ANALYSIS, COHORT SURVIVAL AND TIME ANALYSIS IN THE WEBSTER (1969) STUDY

\begin{tabular}{|c|c|c|c|c|}
\hline Methodologies & $\begin{array}{c}\text { Elementary } \\
\text { Enrollment } 1965\end{array}$ & $\begin{array}{c}\text { Secondary } \\
\text { Enrollment } 1965\end{array}$ & $\begin{array}{l}\text { Elementary } \\
\text { Enrollment } 1968\end{array}$ & $\begin{array}{c}\text { Secondary } \\
\text { Enrollment } 1968\end{array}$ \\
\hline Cohort Survival & $10: 14$ & $13: 12$ & $13: 12$ & $12: 13$ \\
\hline Transition Analysis & $10: 15$ & $15: 10$ & $12: 13$ & 17: 8 \\
\hline Time Analysis & 4: 21 & $18: 7$ & $2: 23$ & $14: 10$ \\
\hline
\end{tabular}

Source: William J. Webster, The Applicability of Selected Ratio and Least Squares Regression Analysis Techniques to the Prediction of Future Educational Attendance Patterns. 
The cases of Kenda1-Upper Rock Spring and Riverside-Dias are interesting. Both Time and Transition Analysis produced close estimates. Tables 1 and 3 show that Time Analysis had the closest estimate with almost 0 correlation. In the case of Kendal, Tables 2 and 4 show that both Time and Transition Analysis produced close estimates on low correlations. Two graphs of the data points for these districts help to explain these low correlations (see Figures 5 and 6).

\section{Selection of a Method}

It will be agreed that, logically, the method which takes into account as many of the variables as possible affecting enrollment would be best. Since Transition Analysis and Cohort Survival take the birth variable into account, either would consequently be preferred. As is seen from the correlations for Transition Analysis, a strong relationship between the independent variable, birth, and the dependent variable, Grade 1, was not established at the district level. Even for the parish level, correlations reached only -.5639 for the 6-5 year leve1 and -.5985 for the 8-7 year level. As already pointed out, low correlations throughout were due to the erratic nature of the data. Orderliness in the data, rather than its variable classification, contributes to efficiency in projections.

Time Analysis is based on the assumption that past trends will, by and large, continue unchanged. Herein lies its weaknesses (and that of a11 regression methods) since it is insensitive to changes affecting future enrollments arising from changed variables such as a change in the birth rate. For selection of a method, it is therefore suggested that Time Analysis be used at district-level and for short-term 


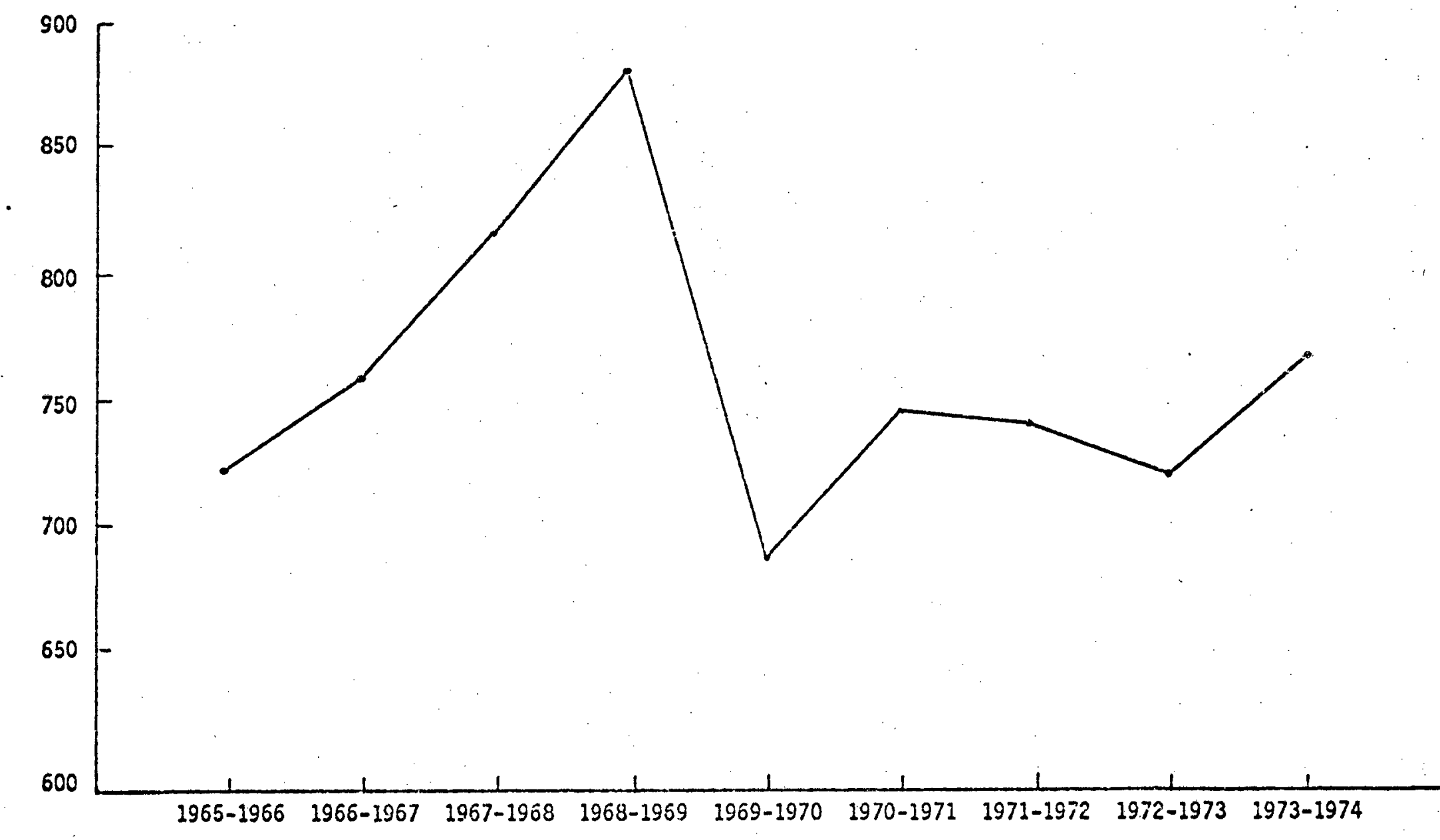

Fig. 5. Graph of Data Points, Time Analysis-Kendal-Upper Rock Spring District. 


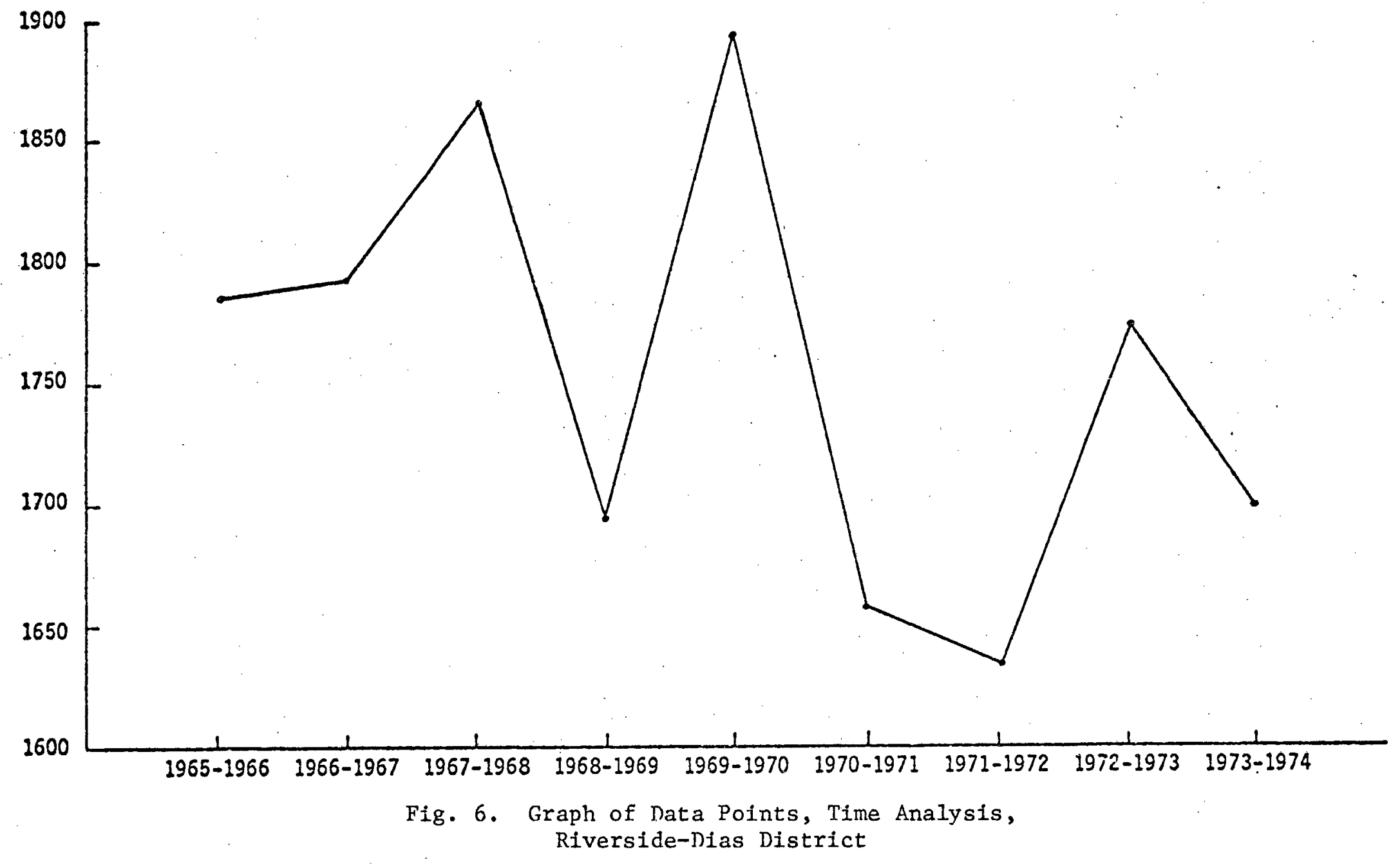


projections. Its results could be modified by estimates on the basis of Cohort Survival or, where more ordered data allows, Transition Analysis. The latter should, for the Jamaican situation, be tested and assessed on figures which show more order than those used in the present study.

\section{Projections}

It was felt that this chapter could usefully conclude with two further analyses:

1. the use of the upper and lower limits in the forecasts, and

2. some projections to 1980 which could be related to projections made by Roberts, et. al. (1974), Jamaican demographers.

Table 8 sets out the upper and lower Iimits for the Time Analysis methodology. The range could be very wide. In cases like Hopewell and Lucea where the data were unreliable or Green Island where there was the unusual growth feature of the opening of a Junior Secondary school, two or three years preceding the year of interest, the range is much wider than desirable for planning. This points to the need for more than statistical estimations to set the parameters in enrollment estimations. Survey methods such as the Bell Telephone or Housing Projection Methods (see Chapter II) or the Delphi Technique (see Appendix E) would prove useful here.

Table 9 sets out the projections to 1980 utilizing Time Ana1ysis and based on the September enrollments, details of which are provided in Appendix G. The data used leaves much to be desired but the projections can perhaps be used as a rough guide. The estimated total for 1981 would be in the region of 19,600 . If the lowest of 
TABLE 8

COMPARISON OF ACTUAL ENROLLMENTS AND 95 PER CENT CONFIDENCE INTERVAL PREDICTIONS FOR THE DISTRICTS BY TIME ANALYSIS BASED ON THE TWO ANTECEDENT PERIODS

\begin{tabular}{|c|c|c|c|c|c|c|c|c|}
\hline \multirow[b]{2}{*}{ Stratum } & \multirow[b]{2}{*}{ District } & \multirow{2}{*}{$\begin{array}{c}\text { Actual Enrollment } \\
1973-1974\end{array}$} & \multicolumn{3}{|c|}{$6-5$ Year Trend } & \multicolumn{3}{|c|}{$8-7$ Year Trend } \\
\hline & & & $\mathrm{LL}$ & $P$ & UL & $\overline{L L}$ & $P$ & UL \\
\hline I & Hopewell & 1979 & 1537 & 2003 & 2470 & 1736 & 1.932 & 2128 \\
\hline II & Sandy Bay & 786 & 651 & 798 & 946 & 721 & 783 & 845 \\
\hline \multirow[t]{2}{*}{ III } & Kendal - Rock & & & & & & & \\
\hline & Spring & 764 & 465 & 767 & 1070 & 609 & 735 & 860 \\
\hline IV & Jericho & 687 & 569 & 733 & 897 & 655 & 724 & 794 \\
\hline Mixed & Lucea & 2177 & 1440 & 1592 & 1744 & 1611 & 1898 & 2186 \\
\hline Mixed & Green Island & 2866 & 2861 & 3248 & 3636 & 2942 & 3136 & 3329 \\
\hline Mixed & Riverside & 1699 & 1331 & 1700 & 2068 & 1494 & 1658 & 1821 \\
\hline Total & Hanover & 14,051 & 13,406 & 14,775 & 16,144 & 13,879 & 14,475 & 15,072 \\
\hline
\end{tabular}

$$
\begin{aligned}
\text { LL } & =\text { Lower Limit } \\
\mathrm{P} & =\text { Predicted } \\
\mathrm{UL} & =\text { Upper Limit }
\end{aligned}
$$


TABLE 9

TIME ANALYSIS PROJECTIONS OF PUPIL POPULATIONS TO 1981 PARISH OF HANOVER, JAMAICA

\begin{tabular}{|c|c|c|c|c|c|c|}
\hline & District & $\begin{array}{c}\text { September Enrollment } \\
1973-1974\end{array}$ & $\begin{array}{l}\text { Projected } \\
1976-1977\end{array}$ & $\begin{array}{l}\text { Projected } \\
1978-1979\end{array}$ & $\begin{array}{l}\text { Projected } \\
1980-1981\end{array}$ & $\begin{array}{l}\text { Projected } \\
1981-1982\end{array}$ \\
\hline 1. & Lucea & 2782 & 2967 & 3258 & 3550 & 3696 \\
\hline 2. & Green Island & 2001 & 2126 & 2152 & 2177 & 2190 \\
\hline 3. & $\begin{array}{l}\text { Kendal - Upper } \\
\text { Rock Spring }\end{array}$ & 756 & 724 & 714 & 704 & 698 \\
\hline 4. & Riverside Dias & 1751 & 1638 & 1595 & 1551 & 1530 \\
\hline 5. & Askenish & 1290 & 1326 & 1419 & 1512 & 1558 \\
\hline 6. & Jericho & 711 & 754 & 775 & 795 & 806 \\
\hline 7. & Cascade & 1203 & 1233 & 1258 & 1283 & 1294 \\
\hline 8. & Sandy Bay & 786 & 933 & 1016 & 1099 & 1141 \\
\hline 9. & Hopewelr & 2031 & 2250 & 2407 & 2565 & 2643 \\
\hline 10. & Gurney's Mt. & 1113 & 1166 & 1208 & 1251 & 1273 \\
\hline 11. & Mt. Peto & 792 & 813 & 823 & 833 & 838 \\
\hline 12. & Flower Hill & 291 & $\mathrm{NF}$ & $\mathrm{NF}$ & $\mathrm{NF}$ & $\mathrm{NF} *$ \\
\hline 13. & Chester Castle & 791 & 919 & 1012 & 1106 & 1153 \\
\hline 14 & Friendship Copse & 432 & 555 & 645 & 735 & 780 \\
\hline & Totals & 16,730 & 17,404 & 18,282 & 19,161 & 19,600 \\
\hline
\end{tabular}

*NF - Not Feasible 
the projections made by Roberts, et. a1. (1974) is used as a gauge and the proportion distribution per parish is assumed as remaining at the 1970 leve1, i.e., 3.15 percent for Hanover, then, by that measure the age group 5-14 at 1980 becoming the 6-15 age group in 1981 might: be in the region of 18,500 .

Whatever projections are made, however, can usefully be checked by the other methods given above. Besides, for planning a year or two in advance, either Cohort Survival or Time Analysis will easily provide useful forecasts provided data, representative of the actual situation, are available and used. 


\section{CHAPTER V}

SUMMARY, CONCLUSIONS AND RECOMMENDATIONS

\section{Summary}

The purpose of this study was to test the efficiency of pupil population projection techniques at micro levels as a basis for educational planning and administration at such levels. The need for such a study and projections was perceived from the Jamaican situation where many schools are cvercrowded, and faced at the beginning of the school year with pressures on accommodation from would-be enrollees. On the other hand, schools in other areas; primarily rural, are underutilized and it has not been determined whether this is due to underpopulation or underattendance.

On the assumption that statistical methods of estimating enrollments are feasible, the relevant literature was reviewed. Several methodologies were identified as being commonly used or in the process of being tried. These all derived from the following statistical techniques:

1. use of ratios (Cohort Surviva1)

2. use of the regression equation (Transition Analysis)

3. use of probabilities and matrix theory (Markov Chain)

4. use of iterative methods (Monte Carlo methods)

A number of methodologies utilizing various factors influencing enrollment or previous features of enrollment have been developed. A11, it 
should be noted, depend on previous trends as probability bases. Three such methods identified as good performers, in a study by Webster (1969), were assessed. These methods and the reasons for their use are listed below:

1. Transition Analysis, a regression method found to perform adequately in one and best in four out of five district types, categorized according to previous population and household growth;

2. Cohort Survival, which performed adequately in three out of the five district types; and

3. Time Analysis, which performed best in one of the district types.

The literature dealing with Jamaican population trends was also reviewed. High birth rates which seem to be declining under campaigns of family planning, low mortality rates, and significant migration rates were among the features described. Population projections based on differing assumptions were also cited.

\section{Conclusions}

Tests of the methodologies were made in seven out of fourteen school districts created in the parish on the basis of physiographic and socioeconomic features lending themselves to discreteness and homogeneity. One year only, 1973-1974, was used as the criterion but two trend periods--a 6-5 year and an 8-7 year antecedent period were used. The methods were also tested on the total figures for the parish.

The data base was weak for projection purposes since end-ofyear figures had to be used. The grade to grade progressions showed up as being unusually erratic; there was missing data in a few cases, 
and at some schools, enrollment data for certain years were simply repetitions of a previous year's data. These data weaknesses may have been due to (1) faulty preparation of, or lack of enrollment returns, (2) irregularity in school attendance and enrollment flowing from lack of enforcement of compulsory education regulations, and/or (3) other social and economic factors contributing to irregular attendance and enrollment.

Against this background, Time Analysis seemed to be the best performer of the three methods at district level in terms of numbers of closest projections overall and projections within a 10 percent range of the actual enrollment figures. Cohort Survival was such a close second that a clear differentiation seemed inadvisable. Transition Analysis seemed to show a tendency to underestimations. Time Analysis tended to overestimate. Cohort Survival seemed responsive to grade by grade fluctuations. All projection methods performed better in districts with few data problems than in districts with considerable data problems. This could be taken to mean that on larger figures and data showing more ordered progressions through grades, Transition Analysis might prove to be more efficient than Time Analysis. This seemed to be proved when parish totals were used. Here, Transition Analysis performed best of the three methods.

As far as projections beyond 1974 were concerned, it was felt that these were risky given the unreliability of the data. One set of projections to 1980-1981 was attempted and assessed against an assumed total for the parish calculated from the 1980 projections made by 
Jamaican demographers and the previous ratio of total population held by the parish.

\section{Recommendations}

Based upon the finds of the researcher and the observations made during and after this study, the following recommendations are made:

1. This study should be replicated in other parishes in Jamaica to test both the performance of the statistical enrollment projection methods and the feasibility of allocating schools and pupil populations to school districts created by natural process.

2. Other statistical methods should be tried, especially those which would take account of the variables affecting enrollment and provide for probability weightings based on different assumptions. The Markov Chain Method seems to be one with very interesting possibilities.

3. A wealth of education data exists in the Jamaican situation. Greater preciseness and care in its preparation and maintenance should be exercised since it could be required at any time for analyses which would be significant to educational research and planning.

4. Enrollment studies of this kind are valuable not only for facilitating planning but also for assessing in which areas of the island compulsory education may feasibly be enforced, or how pupil populations may be distributed within an area where commuting between schools is possible. In addition, the study of enrollment data is useful in pupil accounting generally, as well as in checks on the stability 
of enrollment and attendance at individual schools and in the different grades. Consideration should therefore be given to revising the type of data returns required by the Ministry as well as to ensuring proper returns; for example, submission of beginning year enrollments by grade should be a requirement in addition to the end of year requirements. The former could be expected to represent the peak enrollments, the latter the lowest position.

5. A study of enrollment projections and, in particular, long range projections which are most useful in educational planning cannot depend on statistical forecasting alone. Knowledge of trends, of development potential, of deliberate planning in other than educational areas is necessary. Also necessary is knowledge of popular feeling on the growth directions of the area and people's reaction to this. To mathematical probabilities must be added probabilities based on informed opinion. Methods of securing such opinion to supplement statistical work include The Delphi Technique, a brief description of which is provided in Appendix E. This technique should prove very useful to planners particularly in urban areas.

6. Enrollment studies in Jamaica could usefully include isolation of districts with growth rates of more than 100 percent over a decade, such as was done in the Webster (1969) study. In such cases, local surveys like the Bell Telephone or Housing Projection methods mentioned in the chapter on the review of literature, which assess community growth patterns, might be very useful.

The managerial aspects of educational administration necessarily include estimation of pupil populations at local levels. While this 
might be a hazardous task, it is an important one since it moves toward reducing the unknowns in planning and administration. 
A P P E N D C E S 
APPENDIX A

CENSUS CEASS PROJECTION TABLE 


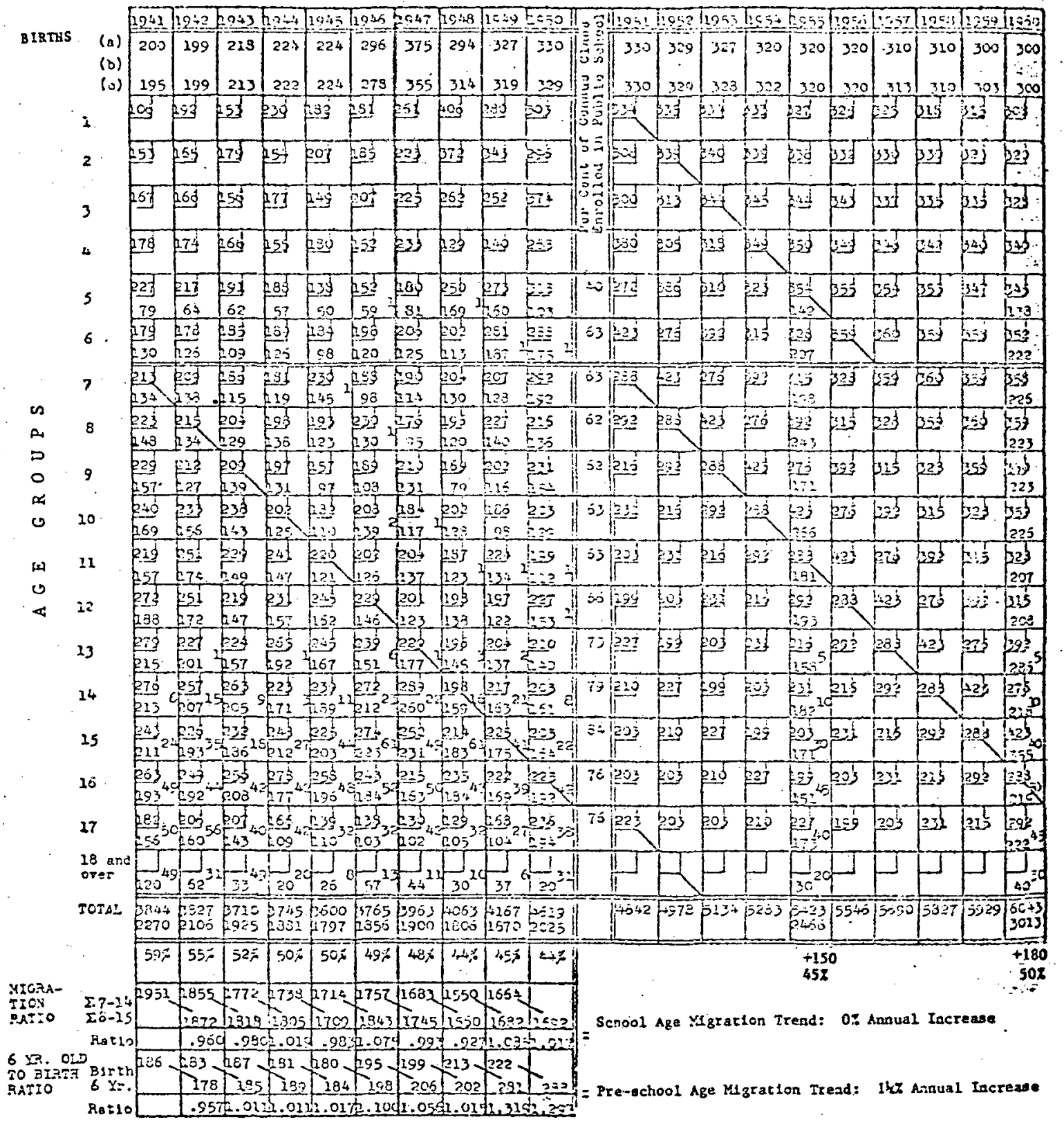

\section{$\checkmark$}

BIRTBS
LEGLND:

BIRTHS (a) - Resizent Births - Ctvil Divisioa (b) - Res1dent B1rths - Scheol Distrlct

(c) - Interpolated B1rths (to School Ceauss Year)

\begin{tabular}{|l|l|l|l}
\hline C & - Census Class (School Census) \\
\hline E & - Resident Enrollment in Publite School
\end{tabular} 8R - Non-zesident Errollment 
APPENDIX B

LIST OF RATIO AND REGRESSION METHODS:

RESULTS OF ANALYSIS

THE WEBSTER (1969) STUDY 
APPENDIX BI

LIST OF RATIO AND REGRESSION METHODS

THE WEBSTER (1969) STUDY 


\section{PROJECTION NETHODOLOGIES EXAMINED BY WEBSTER (1969)}

\section{Name}

1. Cohort-Survival

3. EnrollinentRatio Method I

4. EnrolimentRatio Method II

5. Pupil-Home Un1t

Ratio

6. Time Analysis I

7. Time Analysis II

8. Transition Analysis

9. Birth Analysis

10. Enrollment Regression Analysis I

11. Enrollment Regression Analysis II

Ratio

Rat1o

Ratio

Ratio
Type

Regression

Time

Time

1. Births by City

2. Past District Enrollment

Regression

Births by City

Regression

City School-Age Population

Regression

1. City SchoolAge Population

2. Total City Population

\section{Relationship}

Past tendencles of students to advance from one grade to the next.

Past trends in public school enrollment.

Past ratio of public school enrollment to total city population.

Past ratio of public school enrollment to total county population.

Past ratio of publis school enrollment to city occupied housing units.

Past relationship between public school enrollment and time.

Past relationship between the log of public scheol enrollment and time.

Past tendencles of students to advance from one grade to the next. (Also from birth to kindergarten)

Past relationship between births and public school enrollment.

Past relationship between city school-age population and pubiic school enrollment.

Past relationship between city school-age population, city total population, and puolic school enrollment. 
Name

12. Enrol1ment

Regression

Analysis III

13. Enrollinent

Regression

Analysis IV

14. Housing

Analysis

15. Multi-Variable Analysis I

16. Multi-Variable Analysis II

17. Multi-Variable Analysts III
Tree

Regression

Regression

Regression

Regression

Regression as in 15

Regression
City

2. City SchoolAge Popula-

tion

3. Total City

Population

4. Total County

Population

Same variables

1. City Schoo:Age Popula--

2. Total County Population

City SchoolPopula-

Extrapolated

county

Population

1. Housing

ized Val

tion

Logs of variables used in 15
Relationship

Past relationship between city school-age population, county total population, and public school enrollment.

Past relationship between extrapolated city schoolage population, extrapolated county school-age population, and public school enrollment.

Past relationship between occupied housing, SEV, and public school enrollment.

Past relationship between the predictor vartables and public school enrollments.

Past relationship between the predictor variables and the logs of public school enrollment.

Past relationship between the logs of the predictor variables and the logs of public school enrollment. 
APFENDIX B2

RESULTS OF ANALYSTS

THE WEBSTER (1969) STUDY 
EFFECTIVENESS OF PROJECTION METHODOLOGIES BY STRATA

\section{Projection Methodology}

1. Cohort-Survival Ratio

2. Grade Retention Ratio

3. Enrollment Ratio Method I

4. Enrollment Ratio Method II

5. Pupil-Home Unit (Ratio)

6. Time Analysis I (Regression)

7. Time Analysis II (Regression)

8. Transition Analysis (Regression)

9. Birth Analysis (Regression)

10. Enrollment Regression Analysis I

11. Enrollment Regression Analysis II

12. Enrollment Regression Analysis III

13. Enrollment Regression Analysis IV

14. Housing Analysis (Regression)

15. Multi-Variable Analysis I

16. Multi-Variable Analysis II

17. Multi-Variable Analysis III

\section{Stratum}

A $\quad \underline{C} \quad \underline{D} \quad \underline{E}$

$x \quad 0 \quad x \quad 0 \quad x$

$\begin{array}{lllll}0 & 0 & 0 & 0 & 0\end{array}$

$\begin{array}{lllll}0 & 0 & 0 & 0 & 0\end{array}$

$\begin{array}{lllll}0 & 0 & 0 & 0 & 0\end{array}$

$\begin{array}{lllll}0 & 0 & 0 & 0 & 0\end{array}$

$\begin{array}{lllll}0 & 0 & 0 & \mathrm{~b} & 0\end{array}$

$\begin{array}{lllll}0 & 0 & 0 & \times & 0\end{array}$

$\begin{array}{lllll}b & b & b & x & b\end{array}$

$\begin{array}{lllll}0 & 0 & 0 & 0 & x\end{array}$

$\begin{array}{lllll}0 & 0 & 0 & x & x\end{array}$

$\begin{array}{lllll}0 & 0 & 0 & 0 & 0\end{array}$

$\begin{array}{lllll}0 & \mathrm{x} & 0 & \mathrm{x} & 0\end{array}$

$\begin{array}{lllll}0 & \mathrm{x} & \mathrm{x} & \mathrm{x} & 0\end{array}$

$\begin{array}{ccccc}0 & 0 & 0 & \mathrm{x} & 0\end{array}$

$\begin{array}{lllll}0 & 0 & 0 & 0 & 0\end{array}$

$\begin{array}{lllll}0 & 0 & 0 & 0 & 0\end{array}$

$\underline{0} \quad \underline{0} \quad \underline{0} \quad \underline{0} \quad \underline{0}$

$\begin{array}{lllll}2 & 3 & 3 & 7 & 4\end{array}$

$\mathrm{x}=$ projection methodology is adequate as determined by previously outlined criteria.

$\mathrm{b}=$ projection methodology yields best estimates for Districts in this Strata.

$0=$ projection methodology is not adequate as determined by previously outlined criteria. 
APPENDIX C

POPULATION FEATURES AND PROJECTIONS--JAMAICA 
APPENDIX $\mathrm{Cl}$

PROPORTIONAL DISTRIBUTION OF POPULATION BY PARISH

1921-1970 
PROPORTIONAL DISTRIBUTION OF POPULATION BY PARISH $1921-1970$

\begin{tabular}{lrrrr}
\hline Parish & 1921 & 1943 & 1960 & 1970 \\
\hline Kingston & 7.42 & 8.90 & 7.67 & 5.94 \\
St. Andrew & 6.36 & 10.36 & 18.39 & 2281 \\
St. Thomas & 4.95 & 4.91 & 4.27 & 3.85 \\
Portland & 5.71 & 4.91 & 4.01 & 3.75 \\
St. Mary & 8.32 & 7.35 & 5.85 & 5.43 \\
Si. Ann & 8.27 & 7.77 & 7.10 & 6.63 \\
Trelauny & 4.03 & 3.84 & 3.48 & 3.35 \\
Si. James & 4.89 & 5.14 & 5.16 & 5.56 \\
Hanover & 4.46 & 4.18 & 3.35 & 3.15 \\
Hestmoteland & 8.02 & 7.28 & 6.81 & 6.26 \\
St. Elizatetin & 9.24 & 8.10 & 7.25 & 6.91 \\
Mtrichester & 7.45 & 7.50 & 6.94 & 6.65 \\
Citendon & 9.62 & 9.98 & 10.18 & 9.75 \\
St. Catherine & 11.26 & 9.78 & 9.54 & 9.96 \\
\hline
\end{tabular}

Source: Peicentiges derived from figures in Table 3.1 
APPENDIX C2

PARISH POPULATION CHANGES

$1960-1970$ 
MOVEMENTS IN PARISH POPULATION - 1960-1970

\begin{tabular}{|c|c|c|c|c|c|c|}
\hline \multirow[t]{2}{*}{ PARISH } & \multicolumn{2}{|c|}{ Population } & \multirow{2}{*}{$\begin{array}{l}\text { Intercensual } \\
\text { Increase }\end{array}$} & \multirow{2}{*}{$\begin{array}{l}\text { Natural } \\
\text { lncrease }\end{array}$} & \multirow{2}{*}{$\begin{array}{l}\text { Intcrial } \\
\text { Migrition }\end{array}$} & \multirow{2}{*}{$\begin{array}{r}\text { Extcrnal } \\
\text { Migration }\end{array}$} \\
\hline & 1960 & 1970 & & & & \\
\hline Kizigston & 123.400 & 109.800 & -13.600 & 50,200 & $-28,000$ & $-35,800$ \\
\hline St. Anürew & 296.000 & 421,700 & +125.700 & 132,200 & $+99,100$ & $-106,000$ \\
\hline St. Thomas & $6 \hat{3}, 700$ & 71,200 & $+\quad 2,500$ & 18,200 & $-3,200$ & $-12,500$ \\
\hline Foitland & $6 \div 500$ & 69.300 & $+\quad 4,800$ & 16,800 & $-4,500$ & $-\quad 7,500$ \\
\hline St. Miry & 94,200 & 100,400 & $+6,200$ & 25,500 & $-8,500$ & $-10,800$ \\
\hline S:. Ann & 114.100 & 122,700 & $+8,300$ & 32,300 & $-11,900$ & $-12,100$ \\
\hline Trelawny & 56,100 & 61,900 & $+\quad 5,800$ & 16,500 & $-4,300$ & $-6,400$ \\
\hline St. lames & 83,000 & 103,100 & +20.100 & 30,400 & $+1,200$ & $-11,500$ \\
\hline :Hanove: & 53,900 & 59.400 & $+\quad 5,500$ & 16,300 & $-5,700$ & $-5,200$ \\
\hline Westinorcland & 109,600 & 115,300 & $+6,200$ & 29,700 & $-12,100$ & $-11,400$ \\
\hline St. Elizateth & 116,700 & 127.800 & $+11,100$ & 32,700 & $-13,700$ & $-7,900$ \\
\hline Manchester & 111.800 & 122,900 & $+11,100$ & 35,300 & $-6,400$ & $-17,800$ \\
\hline Clarcrdon & 164,000 & 178,300 & $+11,300$ & 45,100 & $-5,900$ & $-24,800$ \\
\hline St. Catlerine & 153,500 & 184,200 & $+30,700$ & 49,600 & $+3,600$ & $-22,500$ \\
\hline TOTAL & $1,609,800$ & $1,848,500$ & 238.700 & 530.800 & - & $-292,100$ \\
\hline
\end{tabular}

(Totals rounded to neabest 100 )

ESTIMATES OF NET GAIN (+) OR LOSS (-) TO PARISHES AS A RESULT OF INTERNAL MIGRATION 1960-1970

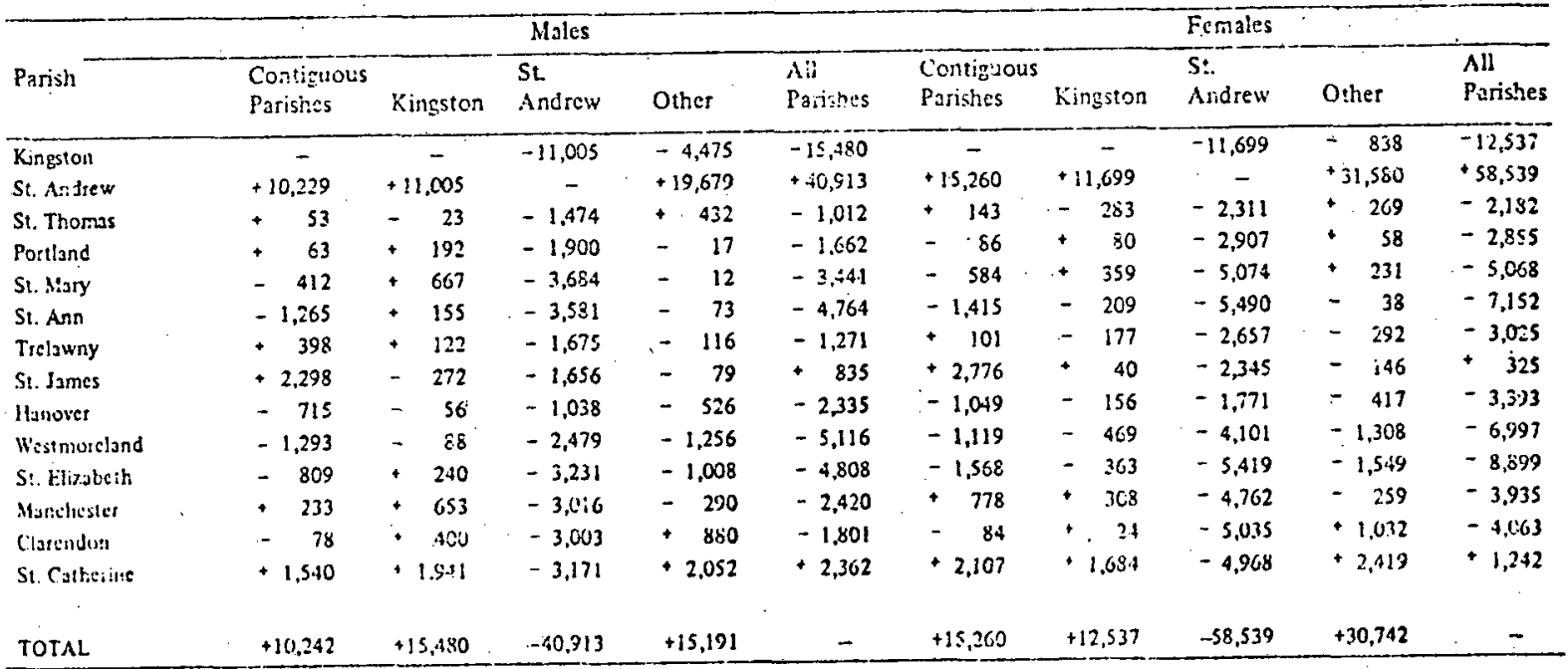


APPENDIX $\mathrm{C} 3$

POPULATION PROJECTIONS - JAMAICA

1975-1990

Roberts et al. (1974) 
SUMMARY OF ESTIMATED POPULATION MOVEMENTS IN JAMAICA, 1960 TO 1990, ACCORDING TO CENSUSES OF 1960 AND 1970, AND THREE PROJECTIONS FROM 1970 TO 1990 (IN OOO's)

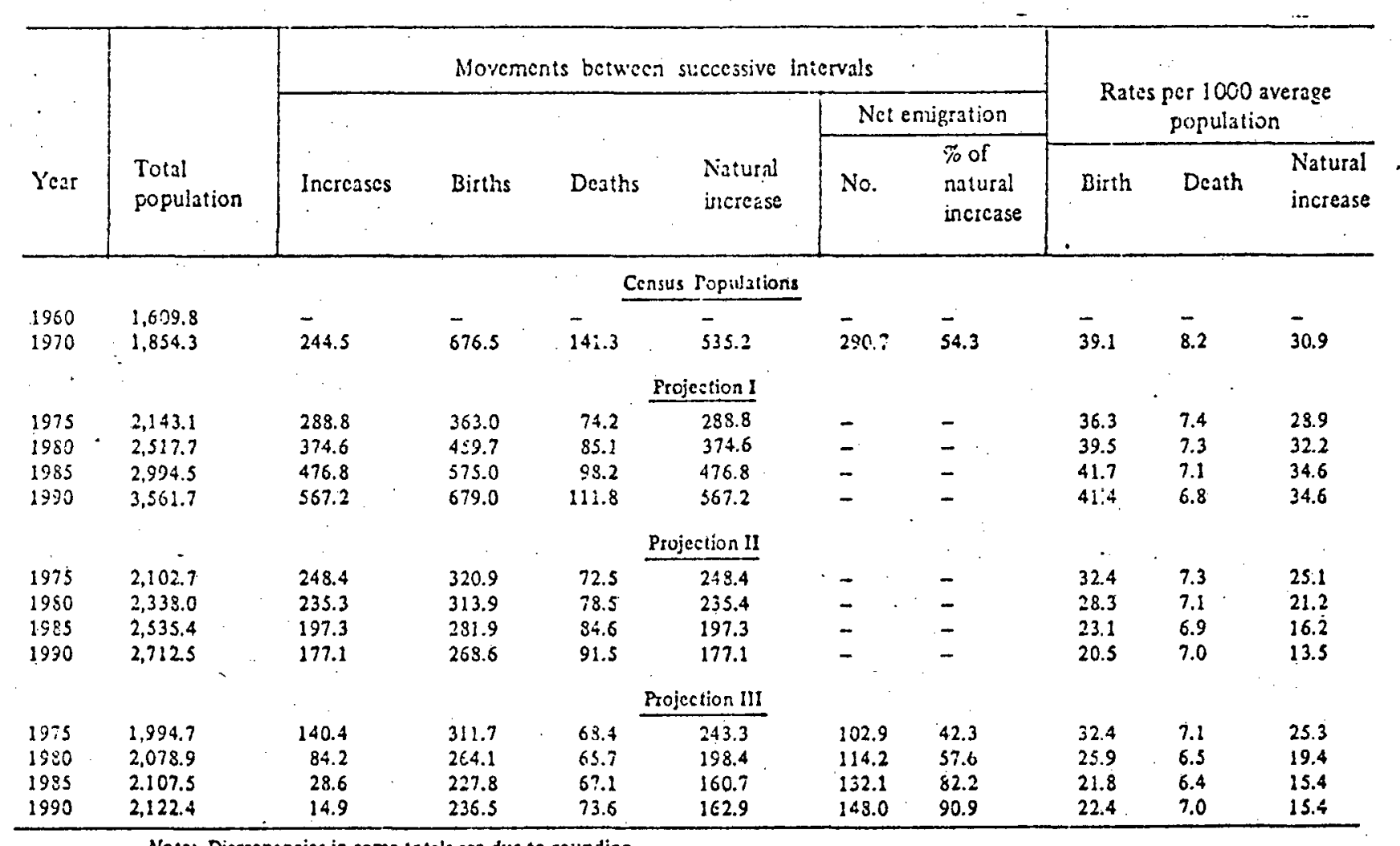


CHANGING AGE STRUCTURE OF THE POPULATION OF JAMAICA TO 1990, ACCORDING TO PROJECTION I (IN OOO's)

\begin{tabular}{|c|c|c|c|c|c|c|c|c|c|c|c|c|}
\hline \multirow{3}{*}{$\begin{array}{l}\text { Age } \\
\text { intervais }\end{array}$} & \multicolumn{6}{|c|}{ Populations } & \multicolumn{6}{|c|}{$\dddot{x}$ distributions } \\
\hline & \multicolumn{2}{|c|}{ Census } & \multicolumn{4}{|c|}{ Projections } & \multicolumn{2}{|c|}{ Cesisus } & \multicolumn{4}{|c|}{ Projections } \\
\hline & 1960 & 1970 & 1975 & 1980 & 1955 & 1990 & 1960 & 1970 & 1975 & 1980 & 1985 & 1990 \\
\hline \multicolumn{13}{|c|}{ Male } \\
\hline D. 4 & 135.0 & 149.0 & 176.3 & 223.3 & 279.3 & 329.8 & 17.5 & 16.5 & 16.8 & $18 . C$ & 18.9 & 18.7 \\
\hline$S-14$ & 197.7 & 280.6 & 3025 & 321.6 & 395.0 & 496.3 & 25.5 & 31.0 & 28.8 & 26.0 & 26.7 & 28.1 \\
\hline Is-44 & 298.6 & 305.9 & 390.0 & $50 ; 3.3$ & 607.2 & 727.1 & .8 .6 & 33.9 & 37.2 & 40.6 & 41.0 & 41.1 \\
\hline $45-54$ & 113.7 & 1.22 .7 & $: 27.2$ & 130.1 & $1: 2.5$ & 14.4 .4 & 14.7 & 13.6 & 12.1 & 10.5 & 8.9 & 8.2 \\
\hline $65+$ & 28.5 & 45.1. & 53.9 & 1 61.1 & 66.6 & 69.6 & 3.7 & 5.0 & 5.1 & 4.9 & 4.5 & 3.9 \\
\hline Tutal & 773.4 & 903.3 & $1,049.9$ & 1.239 .4 & 1,4806 & $i, 767.7$ & 100.0 & 100.0 & 100.0 & 100.0 & 100.0 & 100.0 \\
\hline \multicolumn{13}{|c|}{ Fenale } \\
\hline 04 & 132.9 & 146.8 & 171.6 & 217.3 & 271.8 & 321.0 & 15.9 & 15.4 & 15.7 & 17.0 & 17.9 & 17.9 \\
\hline $5-14$ & 197.0 & 278.0 & 299.0 & $\$ 15.1$ & $\$ 84.9$ & 484.1 & 23.5 & 29.2 & 27.3 & 24.6 & 25.4 & 27.0 \\
\hline $15-44$ & 347.7 & 338.5 & 418.1 & 524.7 & 6.23 .1 & 735.0 & 41.6 & 35.6 & 38.3 & 41.1 & 41.2 & 41.0 \\
\hline 45.64 & 117.6 & 130.7 & 138.5 & 147.5 & 151.5 & 165.4 & 14.1 & 13.8 & 12.7 & 11.5 & 10.0 & 9.2 \\
\hline $65+$ & 41.1 & 57.0 & 66.0 & 73.7 & 82.6 & 88.5 & 4.9 & 6.0 & 0.0 & 5.8 & 5.5 & 4.9 \\
\hline Total & 836.4 & 951.0 & $1,093.2$ & $1,278 .=$ & $1, \leqslant 1 j .9$ & $1,794.0$ & 100.0 & 100.0 & 100.0 & 100.0 & 100.0 & 100.0 \\
\hline
\end{tabular}

Note: This Projection assunnes constani morte'ity and fortility as of $1969-79$ and no external migration. Discrepancies in some totals are due to rounding

POPULATION OF JAMAICA ACCORDING TO 1970 CENSUS AND PROJECTION I (000's)

\begin{tabular}{|c|c|c|c|c|c|c|c|c|c|c|}
\hline \multirow{3}{*}{$\begin{array}{l}\text { Age } \\
\text { iriterval }\end{array}$} & \multicolumn{5}{|c|}{ Male } & \multicolumn{5}{|c|}{ Fomale } \\
\hline & \multirow{2}{*}{$\begin{array}{l}\text { Census } \\
1970\end{array}$} & \multicolumn{4}{|c|}{ Projections } & \multirow{2}{*}{$\begin{array}{l}\text { Census } \\
1970\end{array}$} & \multicolumn{4}{|c|}{ Projections } \\
\hline & & 1975 & 1950 & 1985 & 1900 & & 1975 & 1980 & 1985 & 1990 \\
\hline 0 & 149.0 & 176.3 & 223.3 & 279.3 & 329.8 & 146.8 & 171.6 & 217.3 & 271.8 & 321.0 \\
\hline $5-$ & 155.4 & 147.5 & $174 . .5$ & 221.0 & 276.4 & 154.0 & 145.4 & 170.0 & 215.3 & 269.3 \\
\hline $10-$ & J 25.2 & 155.0 & 147.1 & $.17 \div 0$ & 220.4 & 124.0 & 153.7 & 145.1 & 169.7 & 214.8 \\
\hline $15-$ & 80.7 & 124.7 & 154.3 & 146.5 & 173.3 & 86.0 & 123.6 & 153.2 & 144.7 & 169.1 \\
\hline $20-$ & 60.2 & 80.1 & 123.8 & 15.3 .3 & 145.5 & 68.5 & 85.7 & 123.1 & 152.5 & 144.0 \\
\hline $25-$ & 49.7 & 59.7 & 79.4 & 122.8 & 152.0 & 54.5 & 58.1 & 85.1 & 122.3 & 151.6 \\
\hline $30-$ & 39.3 & 49.2 & $\leq 9.1$ & 78.6 & 121.5 & 43.7 & 54.0 & 67.5 & 84.4 & 121.3 \\
\hline $35-$ & 38.2 & 38.8 & 48.6 & 58.3 & 77.6 & 44.3 & 43.2 & 53.3 & 66.7 & 83.4 \\
\hline 40 & 37.8 & 37.6 & 38.1 & 47.7 & 57.3 & 41.5 & 43.6 & 42.4 & 52.5 & 65.6 \\
\hline $45-$ & 34.0 & 36.8 & 36.6 & 37.1 & 46.4 & 36.7 & 40.6 & 42.5 & 41.4 & $\$ 1.2$ \\
\hline $50-$ & 33.0 & 32.6 & 35.2 & 35.1 & 35.5 & 35.9 & 35.6 & 39.3 & 41.2 & 40.1 \\
\hline s5- & 29.8 & 30.9 & 30.4 & 32.9 & 32.7 & 30.3 & 34.1 & 33.9 & 37.4 & 39.2 \\
\hline $60-$ & 25.8 & 27.0 & 27.9 & 27.5 & 29.7 & 27.8 & 28.2 & .31 .8 & 31.5 & 34.5 \\
\hline cs- & 19.2 & 222 & 23.2 & 24.1 & 23.7 & 20.6 & 25.0 & 25.3 & 28.6 & 28.3 \\
\hline $70 .$. & 12.4 & 15.3 & 17.7 & 18.5 & 19.2 & 14.5 & 17.4 & 21.1 & 21.4 & 24.2 \\
\hline $75+$ & 13.5 & 16.3 & 20.1 & 24.0 & 26.7 & 21.9 & 23.6 & 27.2 & 32.5 & 36.0 \\
\hline Toial & 903.3 & $1,049.9$ & $1,239.3$ & $1,420.6$ & $1,767.7$ & .951 .0 & $1,093.2$ & $1,278.3$ & 1.513 .9 & 1.794 .0 \\
\hline
\end{tabular}


POPULATION. OF JAMAICA ACCCRDING TO 1970 CENSUS AND PROJECTION II $\left(000^{\prime} s\right)$

\begin{tabular}{|c|c|c|c|c|c|c|c|c|c|c|}
\hline \multirow{3}{*}{$\begin{array}{l}\text { Age } \\
\text { interial }\end{array}$} & \multicolumn{5}{|c|}{ Male } & \multicolumn{5}{|c|}{ Female } \\
\hline & \multirow{2}{*}{$\begin{array}{l}\text { Census } \\
1970\end{array}$} & \multicolumn{4}{|c|}{ Projections } & \multirow{2}{*}{$\begin{array}{l}\text { Census } \\
1970\end{array}$} & \multicolumn{4}{|c|}{ Projections } \\
\hline & & 1975 & 1980 & 1985 & 1990 & & 1975 & 1980 & 1985 & 1990 \\
\hline $0-$ & 149.0 & 155.9 & 152.4 & 136.9 & 130.5 & $1+6.8$ & $1 \$ 1.7$ & 148.4 & 133.3 & 127.0 \\
\hline s-- & 55.4 & 147.5 & 154.3 & 150.9 & 135.5 & 154.0 & 145.4 & 150.3 & $1+7.0$ & 132.0 \\
\hline $10-$ & 125.2 & 155.0 & 147.1 & 153.5 & 150.5 & 124.0 & 153.7 & 145.1 & 150.0 & 146.7 \\
\hline 15-. & 80.7 & 124.7 & 154.3 & 146.5 & 153.3 & 86.0 & 123.6 & 153.2 & 144.7 & 149.5 \\
\hline $20-$ & 60.2 & 80.1 & 123.8 & 153.3 & 145.5 & 68.5 & 85.7 & 123.1 & 152.5 & 144.0 \\
\hline 25 & 49.7. & $\$ 9.7$ & .79 .4 & 122.8 & 152.0 & 54.5 & 68.1 & 85.1 & 122.3 & 151.6 \\
\hline 30- & 39.3 & 49.2 & 59.1 & 78.6 & 121.5 & 43.7 & 54.0 & 67.5 & 84.4 & 121.9 \\
\hline $35-$ & 33.2 & 38.8 & 48.6 & 58.3 & $7 \pi 0$ & 44.3 & 43.2 & 53.3 & 66.7 & 83.4 \\
\hline $40-$ & 37.8 & 37.6 & 38.1 & 47.7 & 57.3 & 41.5 & 43.6 & 42.4 & 52.5 & 65.6 \\
\hline $45 \ldots$ & 34.0 & 36.8 & 36.6 & 37.1 & 46.4 & 36.7 & 40.6 & 42.5 & 41.4 & $\$ 1.2$ \\
\hline 50- & 33.0 & 32.6 & 35.2 & 35.1 & 35.5 & 35.9 & 35.6 & 39.3 & 41.2 & 40.1 \\
\hline $5 s-$ & 29.8 & 30.9 & 30.4 & 32.9 & 32.7 & 30.3 & 34.1 & 33.9 & 37.4 & 39.2 \\
\hline $60-$ & 25.8 & 27.0 & 27.9 & 27.5 & 29.7 & 27.8 & 28.2 & 31.8 & 31.5 & 34.8 \\
\hline $65-$ & 9.2 & 22.2 & 23.2 & 24.1 & 23.7 & 20.6 & 25.0 & 25.3 & 28.6 & 28.3 \\
\hline $70-$ & 24 & 15.3 & 17.7 & 18.5 & 19.2 & 14.5 & 17.4 & 21.1 & 21.4 & 24.2 \\
\hline $75+$ & 13.5 & 15.3 & 20.1 & 24.0 & 26.7 & 21.9 & 23.6 & 27.2 & 32.5 & 35.0 \\
\hline Total & 903.3 & $1,029.5$ & $1,148.3$ & $1,248.0$ & 1.337 .5 & 951.0 & $1,073.4$ & $1,189.7$ & $1,287.4$ & $1,375.0$ \\
\hline
\end{tabular}

Victe: Discepancies in some totals are due to rounding.

CHANGING AGE STRUCTURE OF THE POPULATION OF JAMAICA TO 1990, ACCORDING TO PROJECTION II (IN 000's)

\begin{tabular}{|c|c|c|c|c|c|c|c|c|c|c|}
\hline \multirow{3}{*}{$\begin{array}{l}\text { Age } \\
\text { intervals }\end{array}$} & \multicolumn{5}{|c|}{ Populations } & \multicolumn{5}{|c|}{$\%$ distributions } \\
\hline & \multirow{2}{*}{$\begin{array}{l}1970 \\
\text { Census }\end{array}$} & \multicolumn{4}{|c|}{ Projections } & \multirow{2}{*}{$\begin{array}{l}1970 \\
\text { Census }\end{array}$} & \multicolumn{4}{|c|}{ Projections } \\
\hline & & 1975 & 1980 & 1985 & 1990 & & 1975 & 1980 & 1985 & 1900 \\
\hline \multicolumn{11}{|c|}{ Male } \\
\hline 0.4 & 149.0 & 155.9 & 152.4 & 136.9 & 130.5 & 16.5 & 15.1 & 13.3 & 11.0 & 9.8 \\
\hline $5-14$ & 280.6 & 302.5 & 301.4 & 304.8 & 286.0 & 31.0 & 29.4 & 26.3 & 24.4 & 21.4 \\
\hline $15-44$ & 305.9 & 390.0 & 503.3 & 607.2 & 707.0 & 33.9 & 37.9 & 43.8 & 48.7 & 52.8 \\
\hline 45.64 & 122.7 & 127.2 & 130.1 & 132.5 & 144.4 & 13.6 & 12.4 & 11.3 & 10.6 & 10.8 \\
\hline $65+$ & 45.1 & $\leq 3.9$ & 01.1 & 66.6 & 69.6 & 5.0 & 5.2 & 5.3 & 5.3 & 5.2 \\
\hline Total & 903.3 & $1,029.5$ & $1,148.3$ & $1,248.0$ & $1,337.5$ & 100.0 & 100.0 & 100.0 & 160.0 & 100.0 \\
\hline
\end{tabular}

\begin{tabular}{rrrrrrrrrrr}
0.4 & 146.8 & 151.7 & 148.4 & 133.3 & 127.0 & 15.4 & 14.1 & 12.5 & 10.4 & 9.2 \\
5.14 & 278.0 & 299.1 & 295.4 & 297.0 & 273.7 & 29.2 & 27.9 & 24.8 & 23.1 & 20.3 \\
$15-14$ & 338.5 & 418.1 & 524.7 & 623.1 & 715.4 & 35.6 & 39.0 & 44.1 & 18.3 & 52.0 \\
$45-64$ & 130.7 & 138.5 & 137.5 & 151.5 & 165.3 & 13.8 & 12.9 & 12.4 & 11.8 & 12.0 \\
654 & 57.0 & 66.0 & 73.7 & 82.5 & 38.5 & 6.0 & 6.1 & 6.2 & 6.4 & 6.5 \\
Total & 951.0 & $1,073.4$ & $1,189.7$ & $1,287.4$ & $1,375.0$ & 100.0 & 100.0 & 100.0 & 100.0 & 100.0 \\
\hline
\end{tabular}

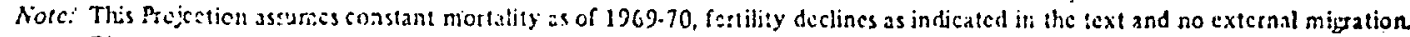
Disercpincies in surne totats are duc to rounding. 
CHANGING AGE STRUCTURE OF THE POPULATTON OF JAMAICA TO 1990, ACCORDING TO PROJECTION III (IN O00's)

\begin{tabular}{|c|c|c|c|c|c|c|c|c|c|c|}
\hline \multirow{3}{*}{$\begin{array}{l}\text { Age } \\
\text { intervals }\end{array}$} & \multicolumn{5}{|c|}{ Populations } & \multicolumn{5}{|c|}{ \%distributions } \\
\hline & \multirow{2}{*}{$\begin{array}{l}1970 \\
\text { Census }\end{array}$} & \multicolumn{4}{|c|}{ Projections } & \multirow{2}{*}{$\begin{array}{l}1970 \\
\text { Cencus }\end{array}$} & \multicolumn{4}{|c|}{ Piojections } \\
\hline & & 1975 & 1980 & 1985 & 1990 & & 1975 & 1080 & 1985 & 1990 \\
\hline \multicolumn{11}{|c|}{ Maic } \\
\hline 0.4 & 149.0 & 151.4 & 128.3 & 110.6 & 115.0 & 16.5 & 15.9 & 13.2 & 11.5 & 12.1 \\
\hline $5-14$ & 280.6 & 301.9 & 296.4 & 275.8 & 235.6 & 31.0 & 31.7 & 30.4 & 28.6 & 24.9 \\
\hline $15-44$ & 305.9 & 328.6 & 384.5 & 427.2 & 460.7 & 33.9 & 34.5 & 39.5 & 44.3 & 48.6 \\
\hline $45-64$ & 122.7 & 116.3 & 103.8 & 86.9 & 72.2 & 13.6 & 12.2 & 10.7 & 9.0 & 7.5 \\
\hline $65+$ & 45.1 & 53.9 & 60.6 & 64.5 & 64.0 & 5.0 & 5.7 & 6.2 & 6.7 & 6.8 \\
\hline Total & 903.3 & 952.1 & 973.6 & 965.0 & 947.5 & 100.0 & 100.0 & 100.0 & 100.0 & 100.0 \\
\hline \multicolumn{11}{|c|}{ Fenaie } \\
\hline 0.4 & 146.8 & 147.4 & 124.8 & 107.7 & 111.9 & 15.4 & 14.1 & 11.3 & 9.4 & 9.5 \\
\hline $5-14$ & 278.0 & 298.7 & 290.7 & 269.0 & 229.8 & 29.2 & 28.7 & 26.3 & 23.5 & 19.6 \\
\hline 15.44 & 338.5 & 395.7 & 478.4 & 550.2 & $6 ! 1.6$ & 35.6 & 38.0 & 43.3 & 48.2 & 52.1 \\
\hline 45.64 & 130.7 & 134.7 & 137.8 & 134.0 & 135.5 & 13.8 & 12.9 & 12.5 & 11.7 & 11.5 \\
\hline $65+$ & 57.0 & 66.1 & 73.6 & 81.6 & 86.1 & 6.0 & 6.3 & 6.7 & 7.2 & 7.3 \\
\hline Total & 951.0 & $1,042.6$ & $1,105.3$ & 1.142 .5 & $1,174.9$ & 100.0 & 100.0 & 100.0 & 100.0 & 100.0 \\
\hline
\end{tabular}

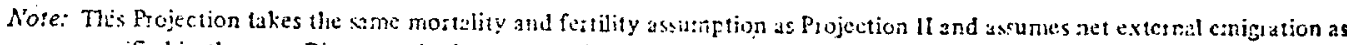
specified in the text. Discreprencies in sore iotils ate uste to rounding.

POPULATION OF JAMAICA ACCORDING TO 1970 CENSUS AND PROJECTION III (000's)

\begin{tabular}{|c|c|c|c|c|c|c|c|c|c|c|}
\hline \multirow{3}{*}{$\begin{array}{l}\text { Age } \\
\text { In:erval }\end{array}$} & \multicolumn{5}{|c|}{ Male } & \multicolumn{5}{|c|}{ Female } \\
\hline & \multirow{2}{*}{$\begin{array}{l}\text { Census } \\
1970\end{array}$} & \multicolumn{4}{|c|}{ Projection } & \multirow{2}{*}{$\begin{array}{l}\text { Census } \\
1970\end{array}$} & \multicolumn{4}{|c|}{ Projection } \\
\hline & & 1975 & 1980 & 1985 & 1999 & & 1975 & 1980 & 1985 & 1990 \\
\hline $\begin{array}{l}0_{-} \\
S_{-}\end{array}$ & $\begin{array}{l}149.0 \\
155.4\end{array}$ & $\begin{array}{l}151.4 \\
147.5\end{array}$ & 128.3 & 110.6 & 115.0 & 146.8 & 147.4 & 124.8 & 107.7 & $1 ! 2.0$ \\
\hline $\begin{array}{r}5- \\
10-\end{array}$ & $\begin{array}{l}155.4 \\
125.2\end{array}$ & $\begin{array}{l}147.5 \\
154.4\end{array}$ & $\begin{array}{l}149.9 \\
146.5\end{array}$ & 127.0 & 109.5 & 154.0 & 145.4 & 146.0 & 123.7 & 106.7 \\
\hline 15 & $\begin{array}{r}125.2 \\
80.7\end{array}$ & $\begin{array}{l}154.4 \\
121.6\end{array}$ & $\begin{array}{l}146.5 \\
149.9\end{array}$ & 143.9 & 126.1 & 124.0 & 153.3 & $144 . ?$ & 145.3 & 123.1 \\
\hline $20-$ & 60.2 & $\begin{array}{r}12.0 \\
69.9\end{array}$ & $\begin{array}{l}149.9 \\
105.3\end{array}$ & 142.3 & $1 \div 4.6$ & 86.0 & 122.1 & 150.9 & 142.5 & 143.1 \\
\hline $25 \ldots$ & 49.7 & 4.4 .5 & $\begin{array}{r}105.3 \\
51.7\end{array}$ & $\begin{array}{r}129.8 \\
778\end{array}$ & 123.2 & 68.5 & 81.7 & 116.0 & 14.3 .4 & 135.4 \\
\hline $30-$ & 39.3 & $\$ 4.4$ & $\begin{array}{l}51.7 \\
30.8\end{array}$ & 77.8 & 96.0 & $\leq 4.5$ & 62.4 & 74.5 & 105.6 & 130.6 \\
\hline 35- & 38.2 & 28.2 & $\begin{array}{l}30.8 \\
24.7\end{array}$ & 35.7 & 53.9 & 43.7 & 48.9 & 56.0 & 66.8 & 94.8 \\
\hline 40-. & 37.8 & 30.0 & $\begin{array}{l}24.7 \\
22.1\end{array}$ & 22.1 & 25.7 & 44.3 & 39.7 & 44.4 & 50.8 & 60.7 \\
\hline $45-$ & 34.0 & 31.3 & $\begin{array}{l}22.1 \\
24.8\end{array}$ & 19.4 & 17.4 & 41.5 & 41.0 & 36.7 & 41.0 & 47.0 \\
\hline so- & 33.0 & 29.5 & 27.2 & 18.3 & 16.1 & 36.7 & 38.8 & 38.2 & 34.3 & 38.3 \\
\hline $55-$ & 29.8 & 29.2 & 26.1 & 21.6 & 15.9 & 35.9 & 34.6 & 36.5 & 36.0 & 32.3 \\
\hline $60-$. & 25.8 & 26.3 & 25.7 & 24.0 & 19.0 & 30.3 & 33.5 & 32.3 & 34.1 & 3.36 \\
\hline 65 - & 19.2 & 22.2 & 226 & 23.0 & 21.2 & 27.8 & 27.8 & 30.8 & 29.7 & 31.3 \\
\hline 70 & 12.4 & 15.4 & 17.8 & 22.2 & 19.8 & 20.6 & 25.0 & 25.0 & 27.7 & 26.7 \\
\hline $75+$ & .13 .5 & 16.3 & 20.2 & 18.1 & 17.7 & 14.5 & 17.4 & 21.1 & 21.2 & 23.4 \\
\hline Total & $90 \hat{3} .3$ & 952.1 & 973.6 & $\begin{array}{r}24.2 \\
23650\end{array}$ & 26.5 & 21.9 & 23.9 & 27.5 & 32.8 & 36.1 \\
\hline & & & & 965.0 & 947.5 & 951.0 & 1.042 .6 & $1,105.3$ & $1,142.5$ & $1,174.9$ \\
\hline
\end{tabular}


APPENDIX C4

POPULATION PROJECTIONS - JAMAICA

$1970-2020 \mathrm{AD}$

Walsh (1970) 
DEMOGRAPHIC PROJECTIONS OF JAMAICA, 1970-2020, UNDER VARIOUS FERTILITY ASSUMPTIONS (IN MILLIONS)

$\begin{array}{lllll}\text { Year } & \text { High } & \text { Medium } & \text { Low } & \text { Very Low } \\ 1970 & 2.093 & 2.093 & 2.093 & 2.093 \\ 1980 & 2.995 & 2.942 & 2.888 & 2.834 \\ 1990 & 4.361 & 4.069 & 3.794 & 3.512 \\ 2000 & 6.394 & 5.503 & 4.719 & 3.849 \\ 2010 & 9.992 & 7.190 & 5.818 & 4.055 \\ 2020 & 13.936 & 8.985 & 7.004 & 4.136\end{array}$


APPENDIX D

DESCRIPTION OF SCHOOL DISTRICTS 


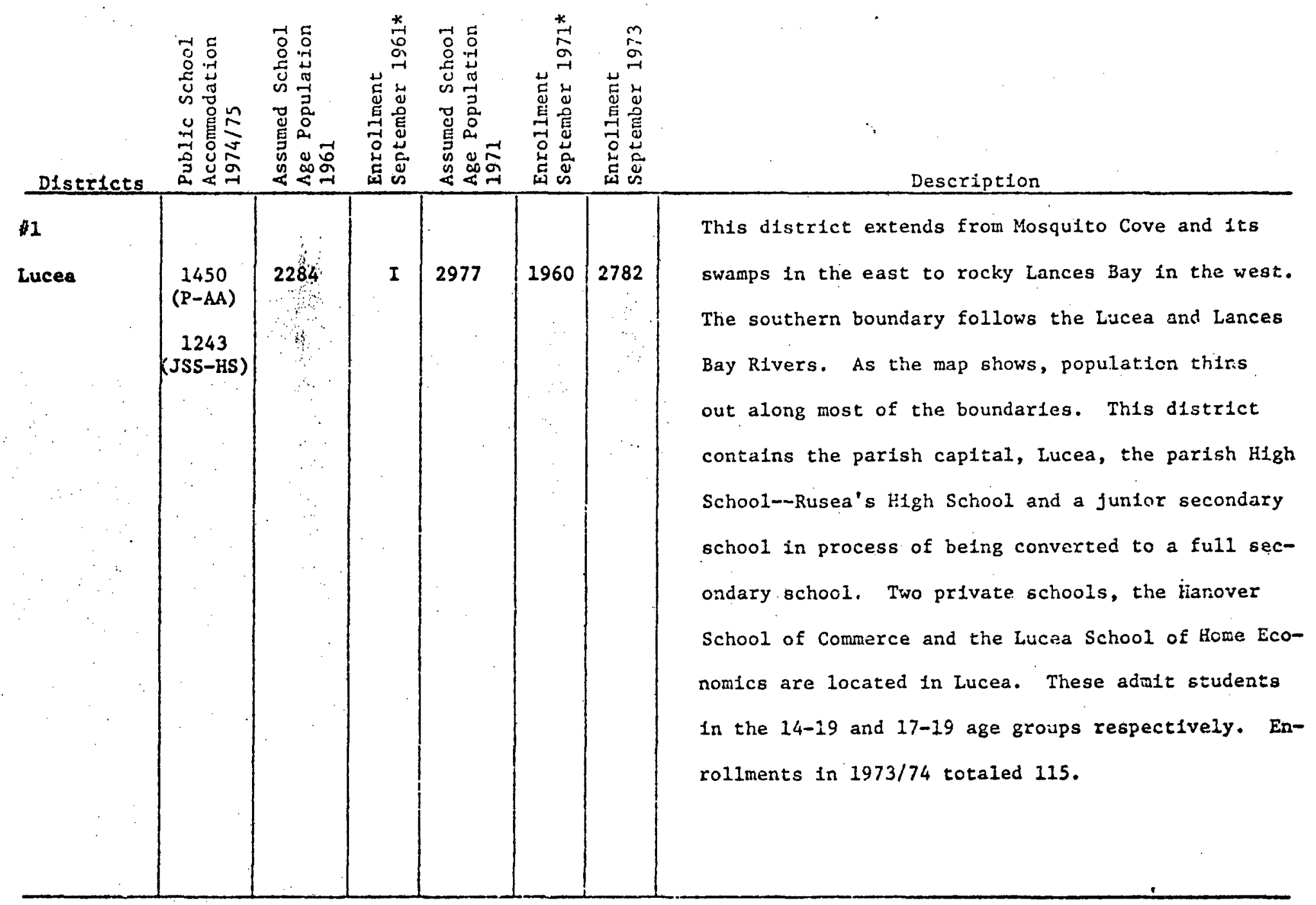

*Assuned school age population 1961, related to 5-14 age group in 1960 Census *Assumed school age population 1971, related to 5-14 age group in 1970 Censtis

I - Incomplete-data missing

JSS - Junfor Secondary School; HS - High School; P - Prinlary; AA - All Age 


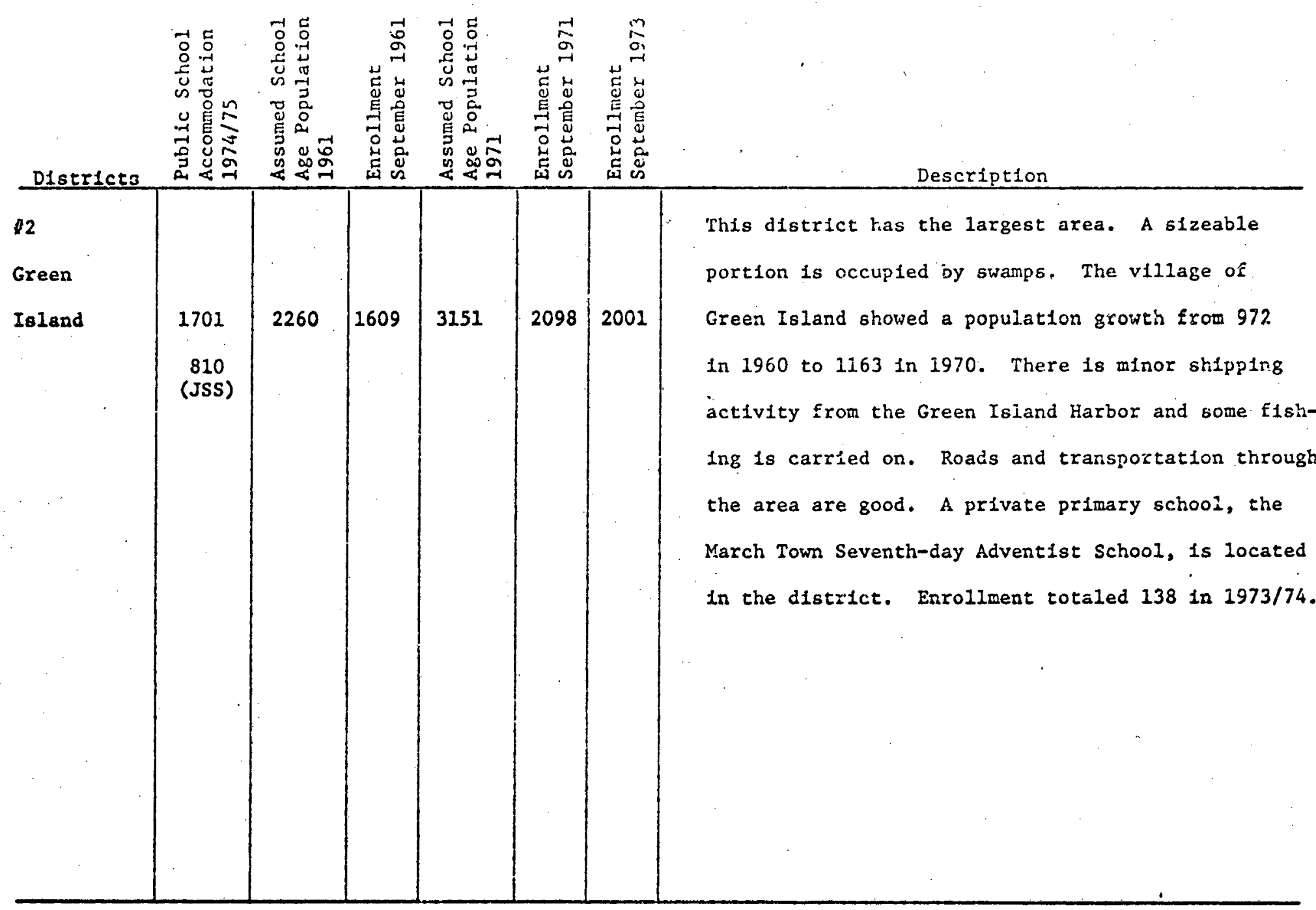




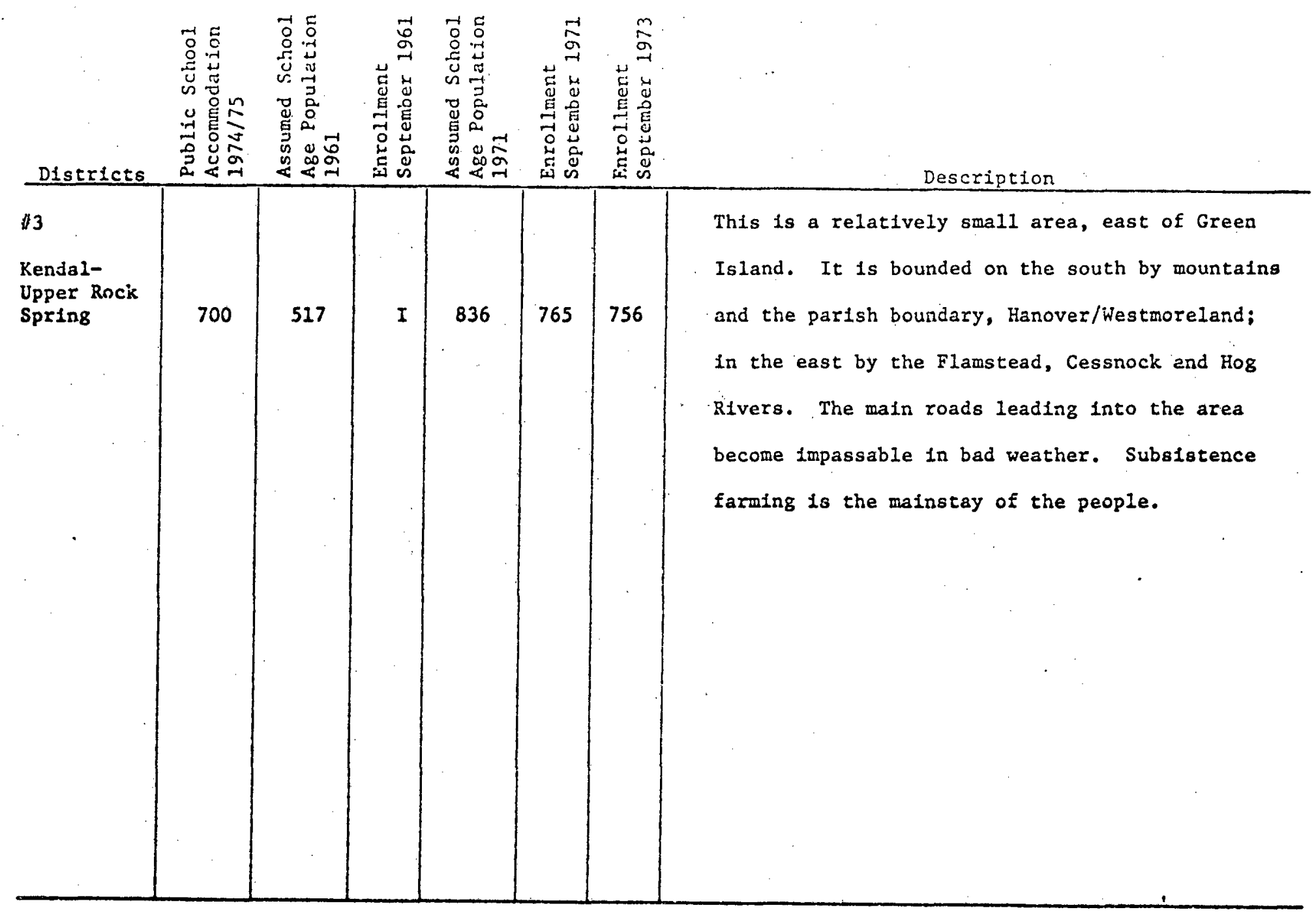




Riverside-




Chambers Pen




Cascade




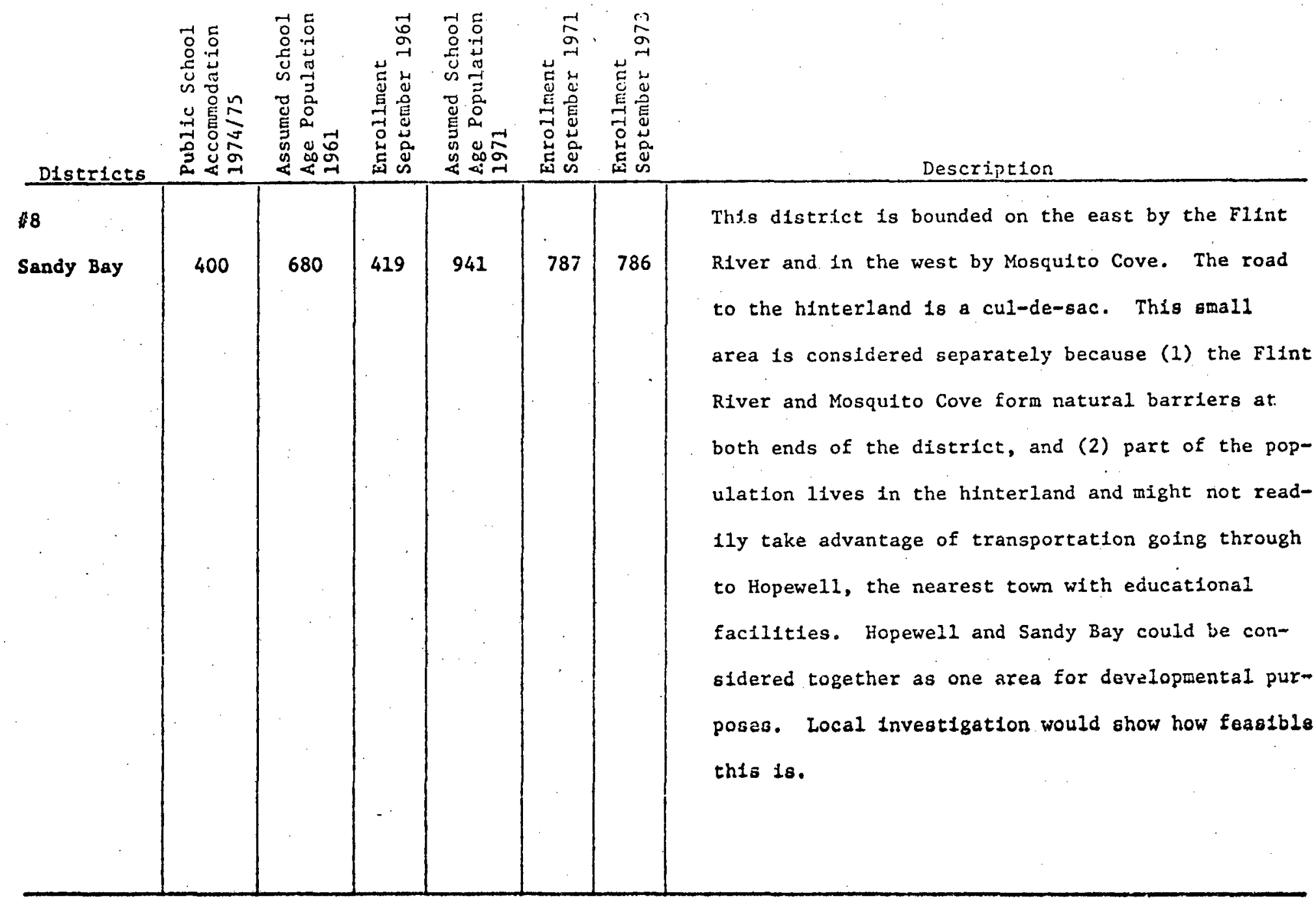




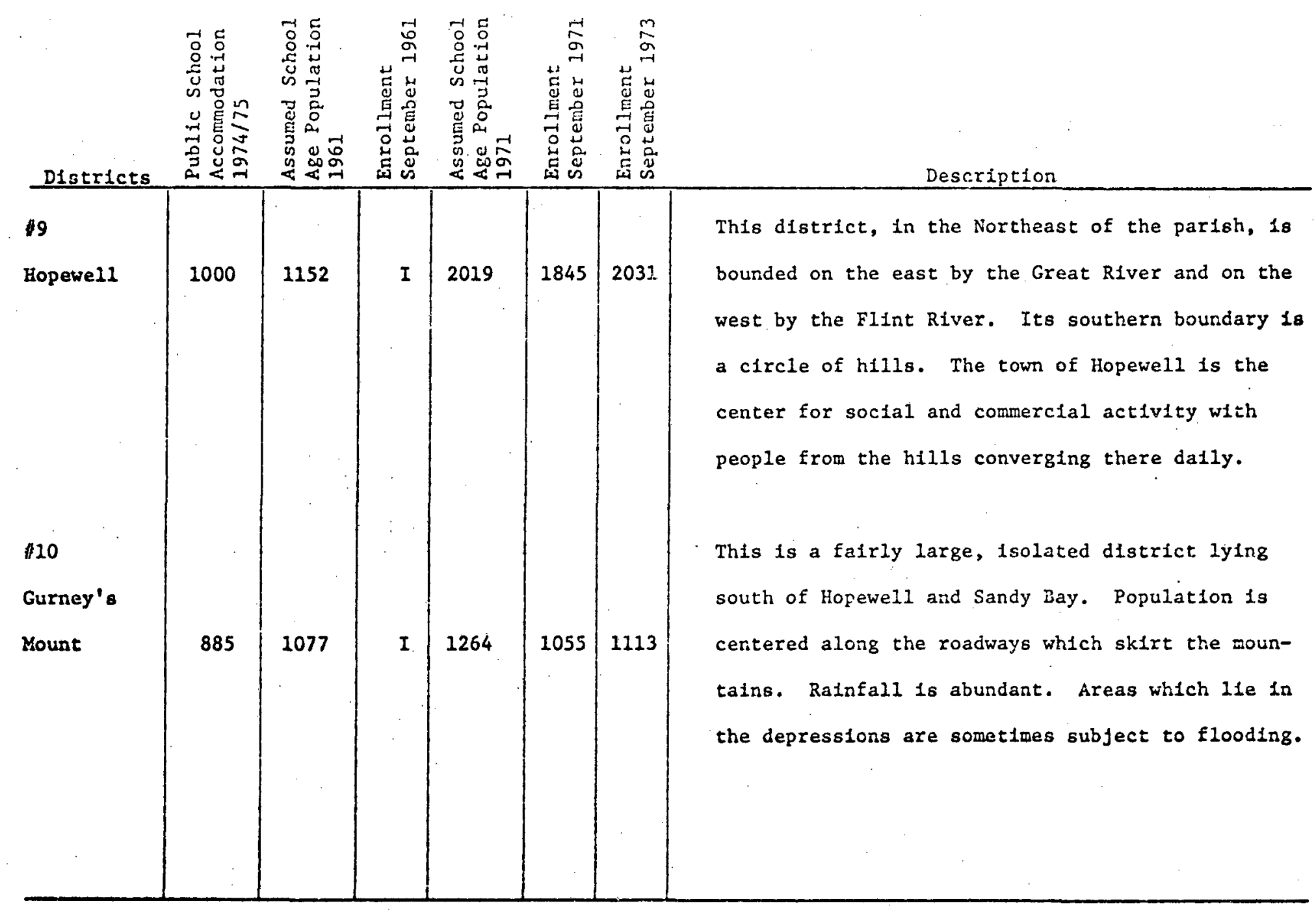




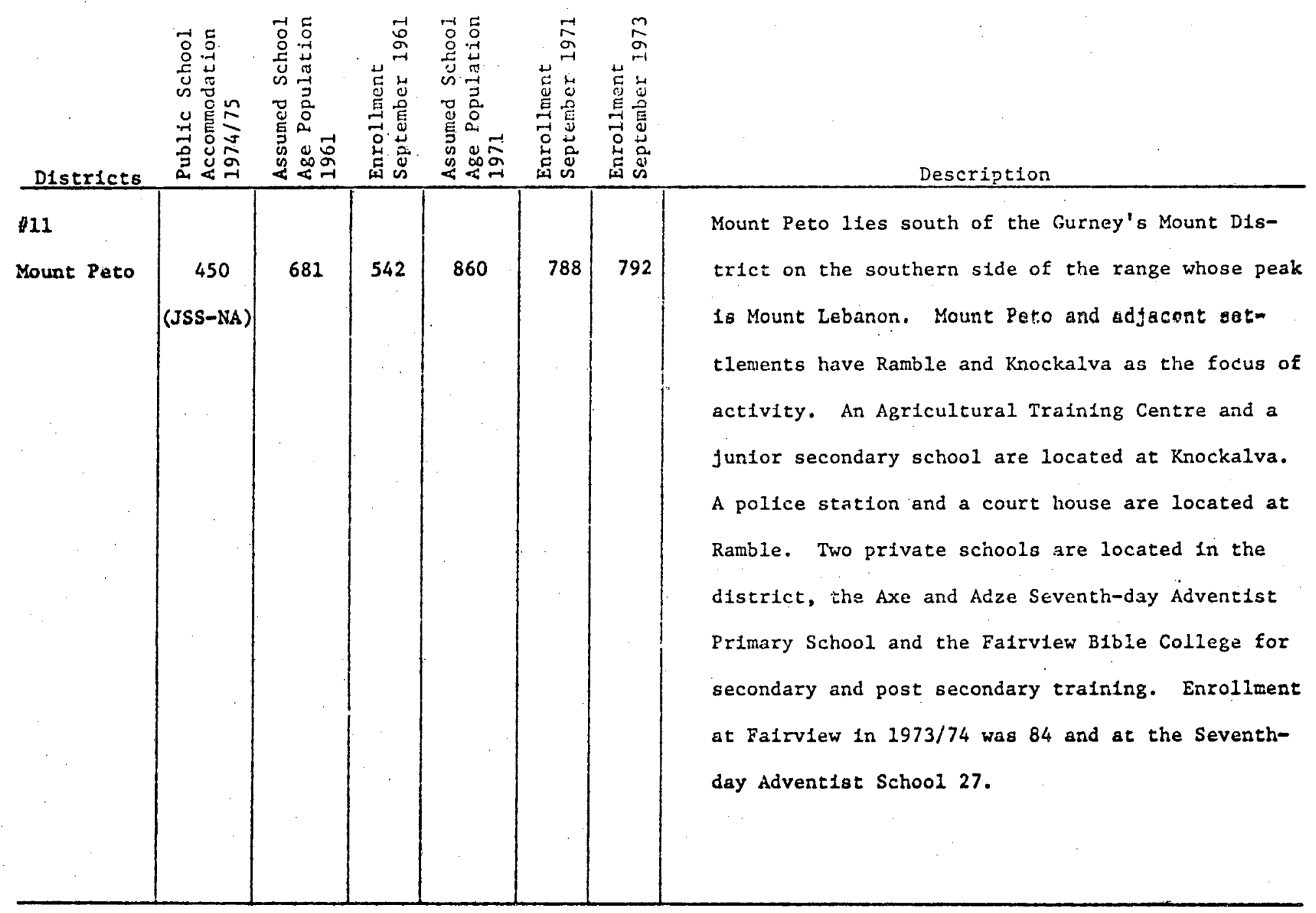

NA - Not Ava1lable

JSS = Jun1or Secondary School 


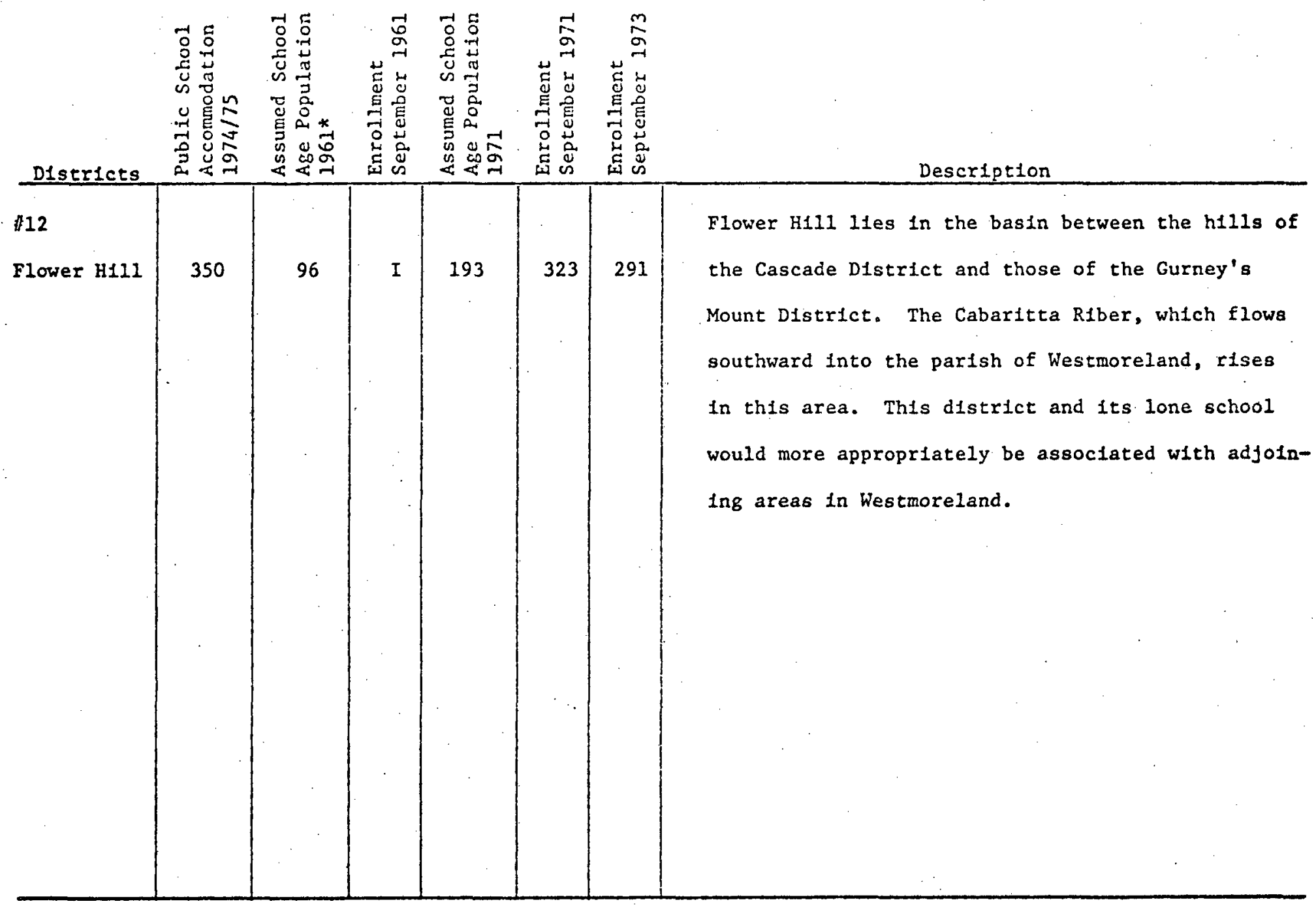




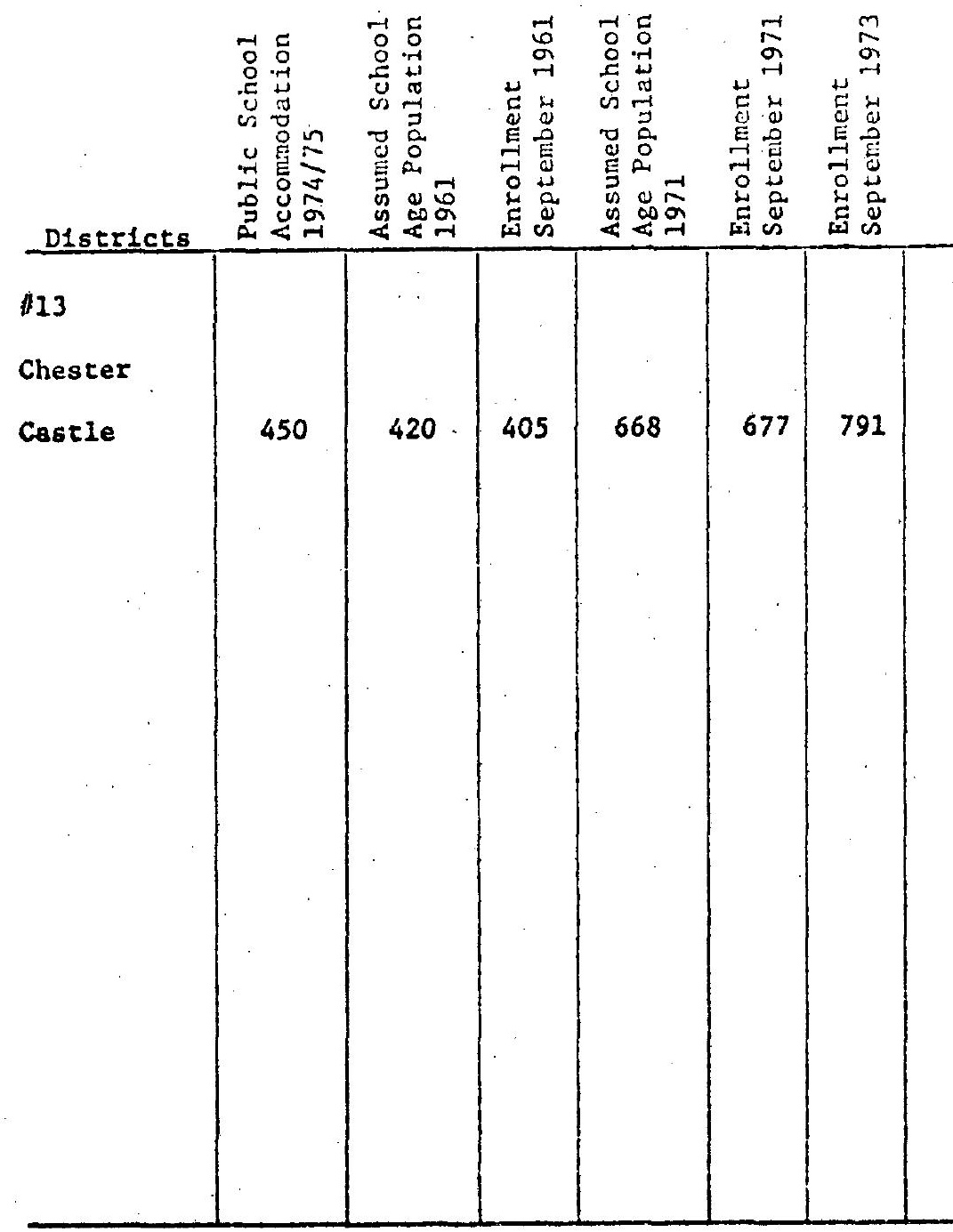


Chester Castle, although apparently contiguous to the Mount Peto D1strict, is separated from it by a large property. One of 1ts two schools, the Senior School, is compietely 1solated and its development a question of some difficulty. Oniy a river with no bridge separates it from the areas in the adjoining parlsh with which it would be better assoclated. 


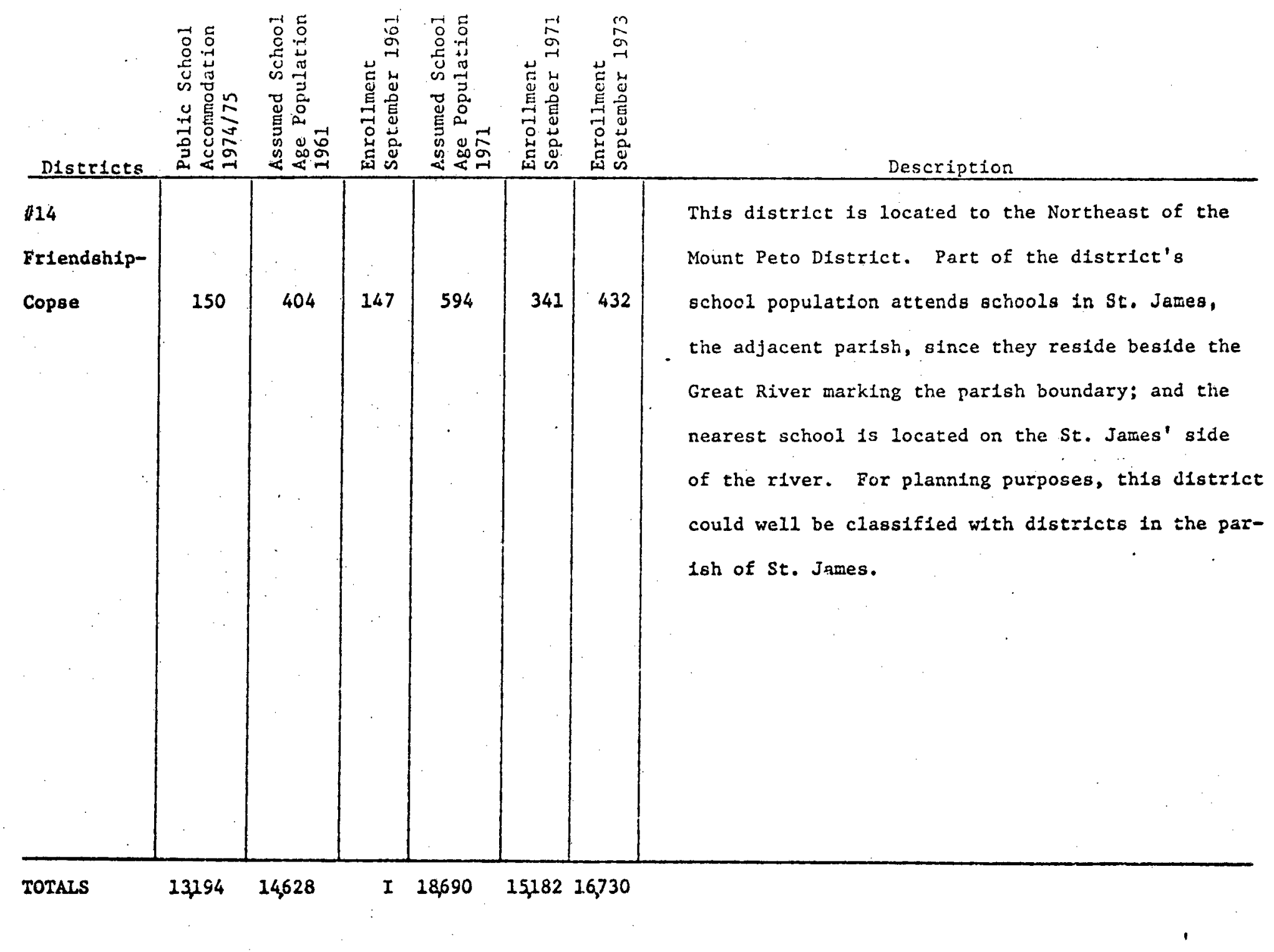


APPENDIX E

THE DELPHI TECHNIQUE 
The Delphi is a relatively recently developed technique belonging to the family of think tank procedures. It was popularized by the RAND Corporation (circa 1964) which used it in a long range forecasting study of technological breakthroughs. It is aimed at identifying the probability of events occuring in the future and at how to incorporate variables such as technological innovations and social values into planning and organization.

The Delphi relies on group judgments on factual matters, in the absence of precise information and seeks to correct this information objectively before it is finally used. It may be a tool for decision making or information building.

It is distinguished from other group consultation mechanisms by

1. its anonymity

2. its iterative approach

3. its advantages over other mthods of group consultation.

Its anonymity arises from the method of securing informationthat is, through written correspondence.

It is iterative in nature because first round information is relayed to participants, for second opinions, in the light of group views. A third or fourth consultation may take place.

Its advantages over other methods of group consultation include the following:

1. variable numbers of people with varied skills may be consuited;

2. the lack of group pressures as in a face to face situation; 
102

3. a variable number of questions or issues may be posed at the same time;

4. Its flexibility. Delphi may be conducted in a group session or on a personal interview basis. If more than 15 members are involved, questionaires may be mailed to participants with prepaid envelopes for returns;

5. computerized approaches may be used when time is important;

6. the method fosters group consensus;

7. through its anonymity it may bring greater rationality to the decision making process.

Its major limitation may be the time that is involved. A three round Delphi may take up to three months. This points to the necessity for quick follow-up and correct estimate of manpower requirements to conduct the particular Delphi.

Skutsch and Hall (1973) ${ }^{1}$ regarded the Delphi as most useful in the following areas of educational planning:

1. human relations

2. provisions of facilities and services

3. curriculum plannirg

4. evaluation

5. business operations

Its potential usefulness in such areas as population projections, planning, designing and location of school buildings is stressed. They also point out that present methods of securing community participation,

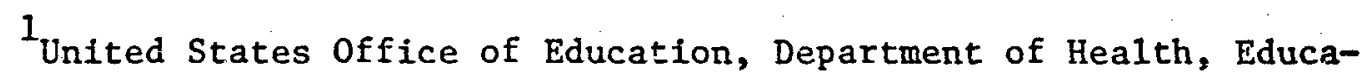
tion and Welfare. Delphi: Potential Uses in Educational Planning by Margaret Skutsch and Diana Hall (July, 1973). 
for example, citizens commttees, public opinion polls or surveys, utlization of administrators, sensitive to people's wishes, may be augmented by the use of the Delphi. 
APPENDIX $\mathrm{F}$

COMPUTER PRINTOUTS OF STATISTICS

USED IN THE STUDY 
APPENDIX F1

TIME ANALYSIS

EIGHT YEAR TREND

SEVEN DISTRICTS 


\section{TIME ANALYSTS : 8 YEAR TREND \\ DISTRICT \#9, HOPEWELL \\ (STRATUM \#I)}

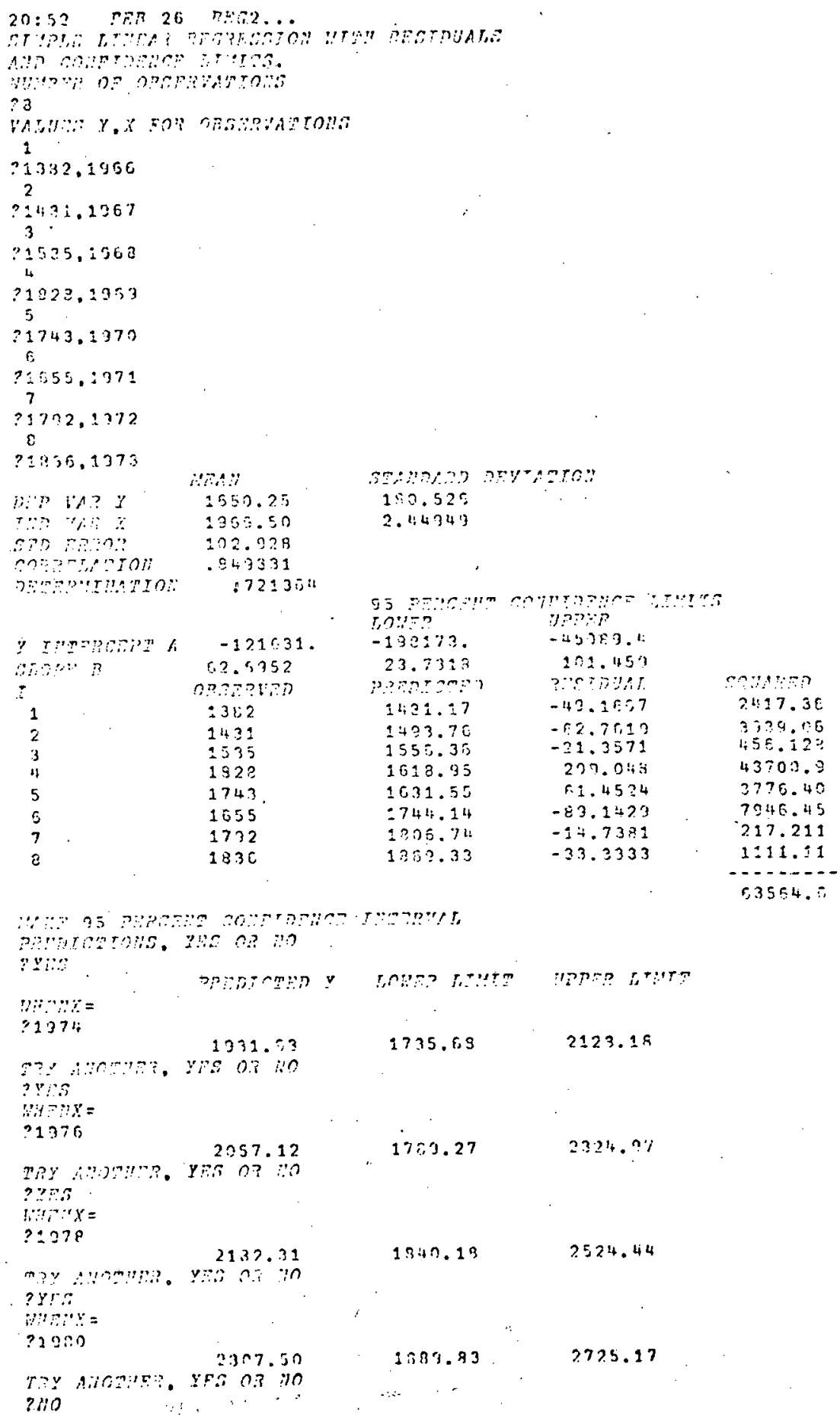


TIME ANALYSIS: 8 YEAR TREND

DISTRICT \# 8 , SANDY BAY

(STRATUM 非I)

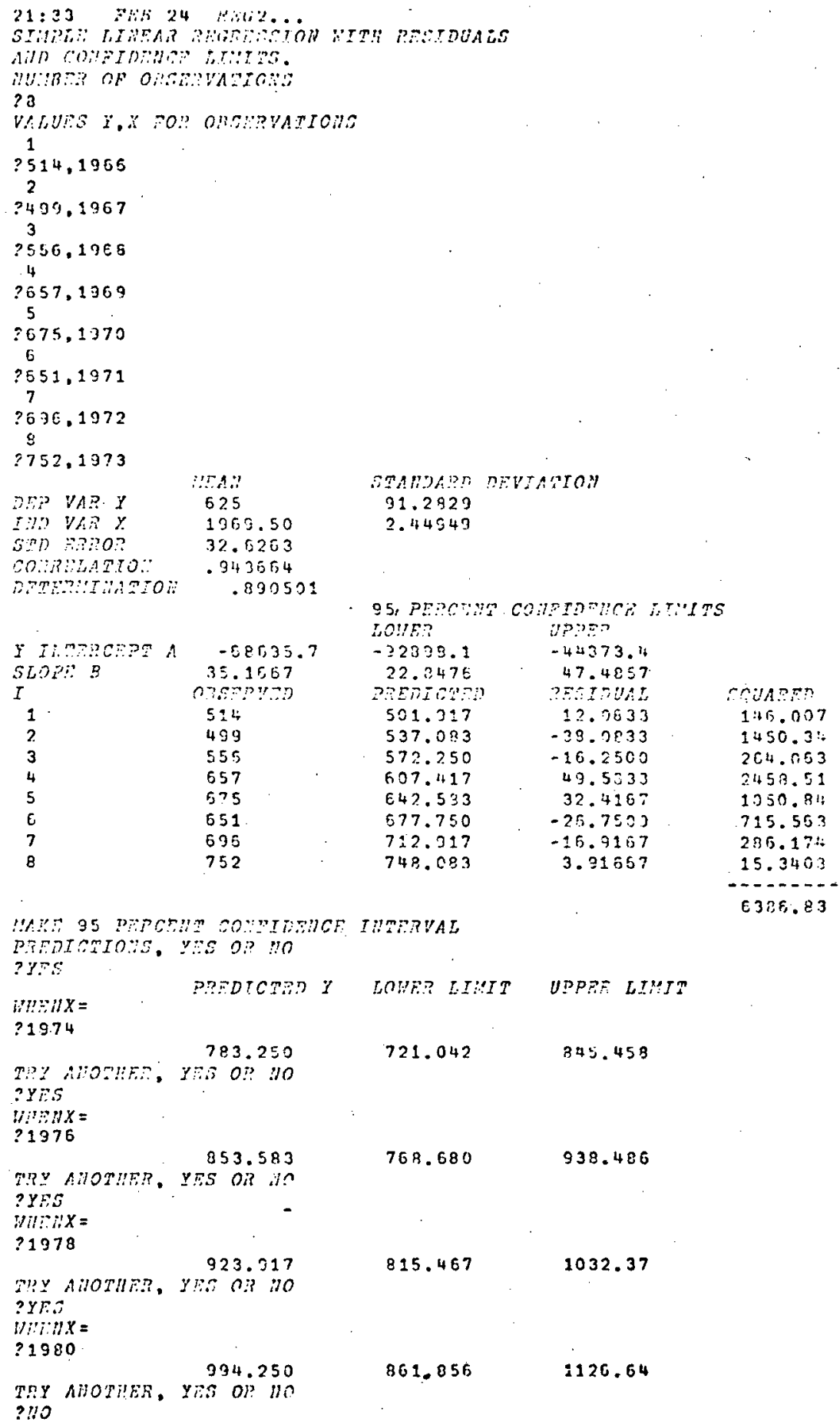


TIME ANALYSIS: 8 YEAR TREND DISTRICI \#3, KENDAL-UPPER ROCK SPRING (STRATUM 非 III)

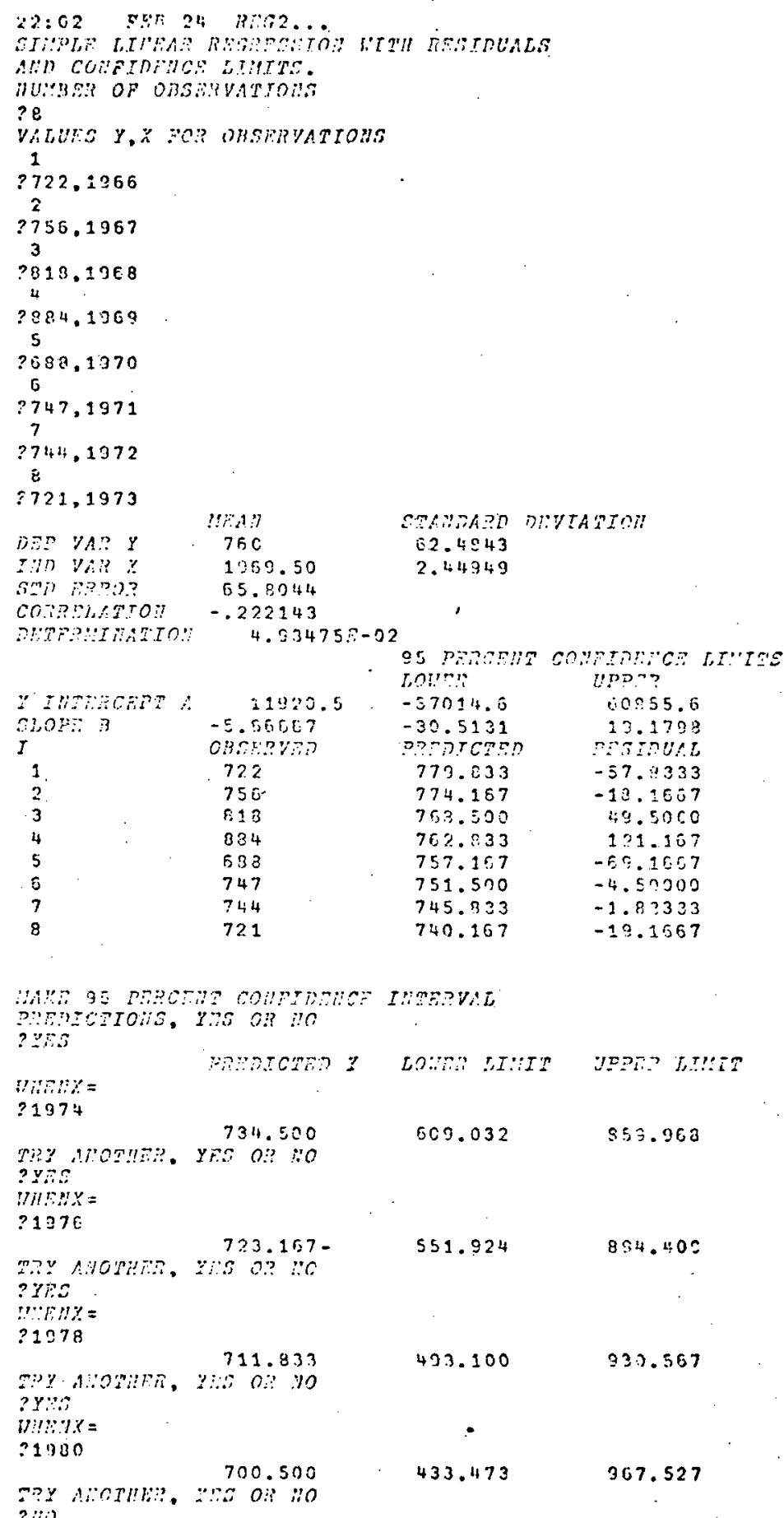

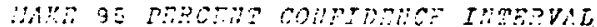

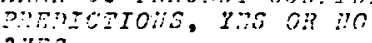

295

$\operatorname{mans}=$

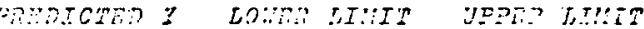

21974

734.500

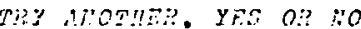

553.963

?Y:S

IIIT:Le

$? 127 \varepsilon$

$723.157-$

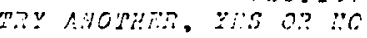

?TRE

".:11:1\%=

$? 1278$

ay dion?

? $y 20$

in:m:x =

$: 1300$

-7y

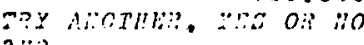

854.400 


\section{TIME ANALYSIS : 8 YEAR TREND DISTRICT 非, JERICHO \\ (STRATUM \#IV)}

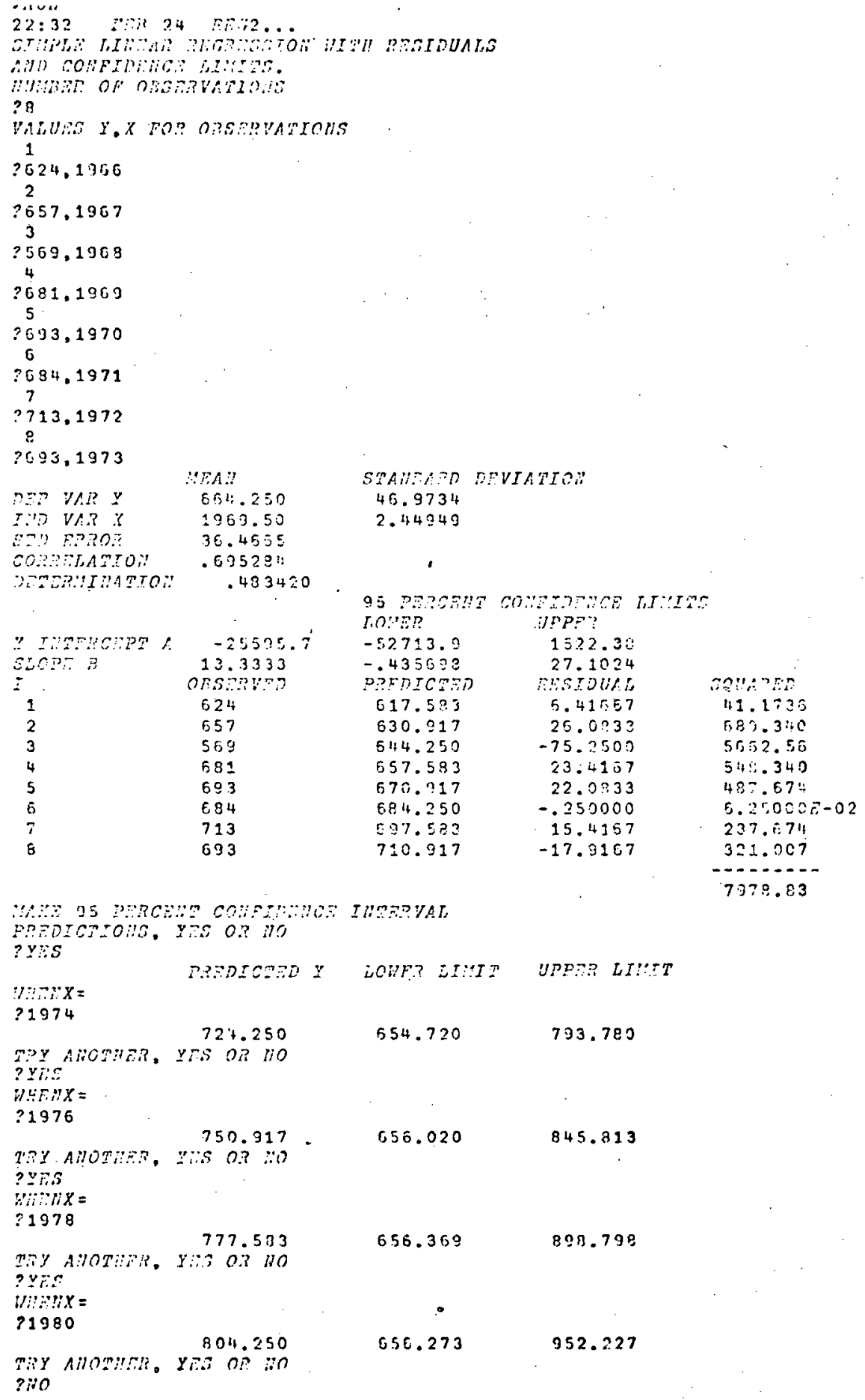


TIME ANAIYSIS: 8 YEAR TREND

DISTRICT '\# 1 , LUCEA

(MIXED STRATUM)

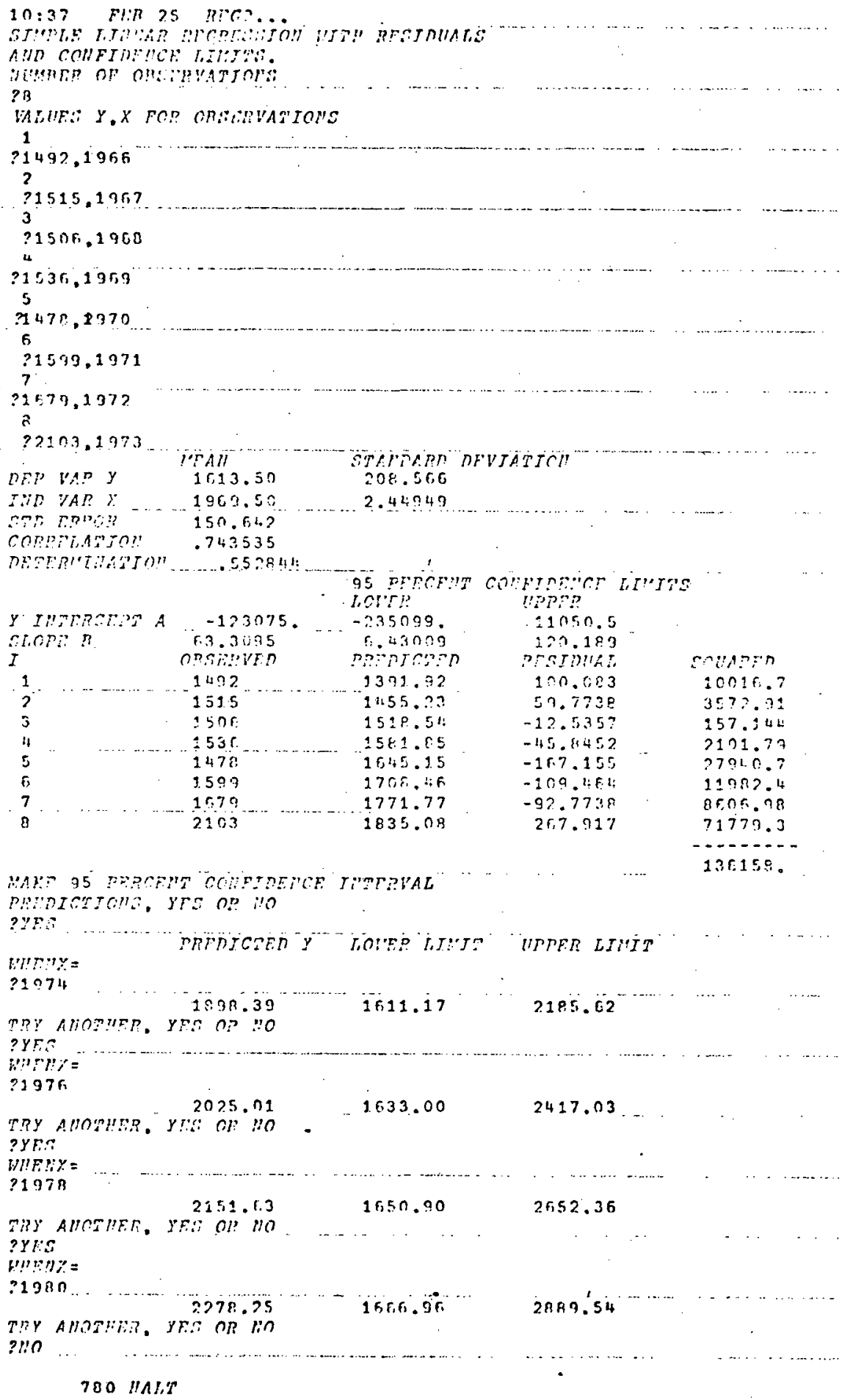


TIME ANALYSIS : 8 YEAR TREND

DISTRICT 非, GREEN J.SLAND

(MIXED STRATUM)

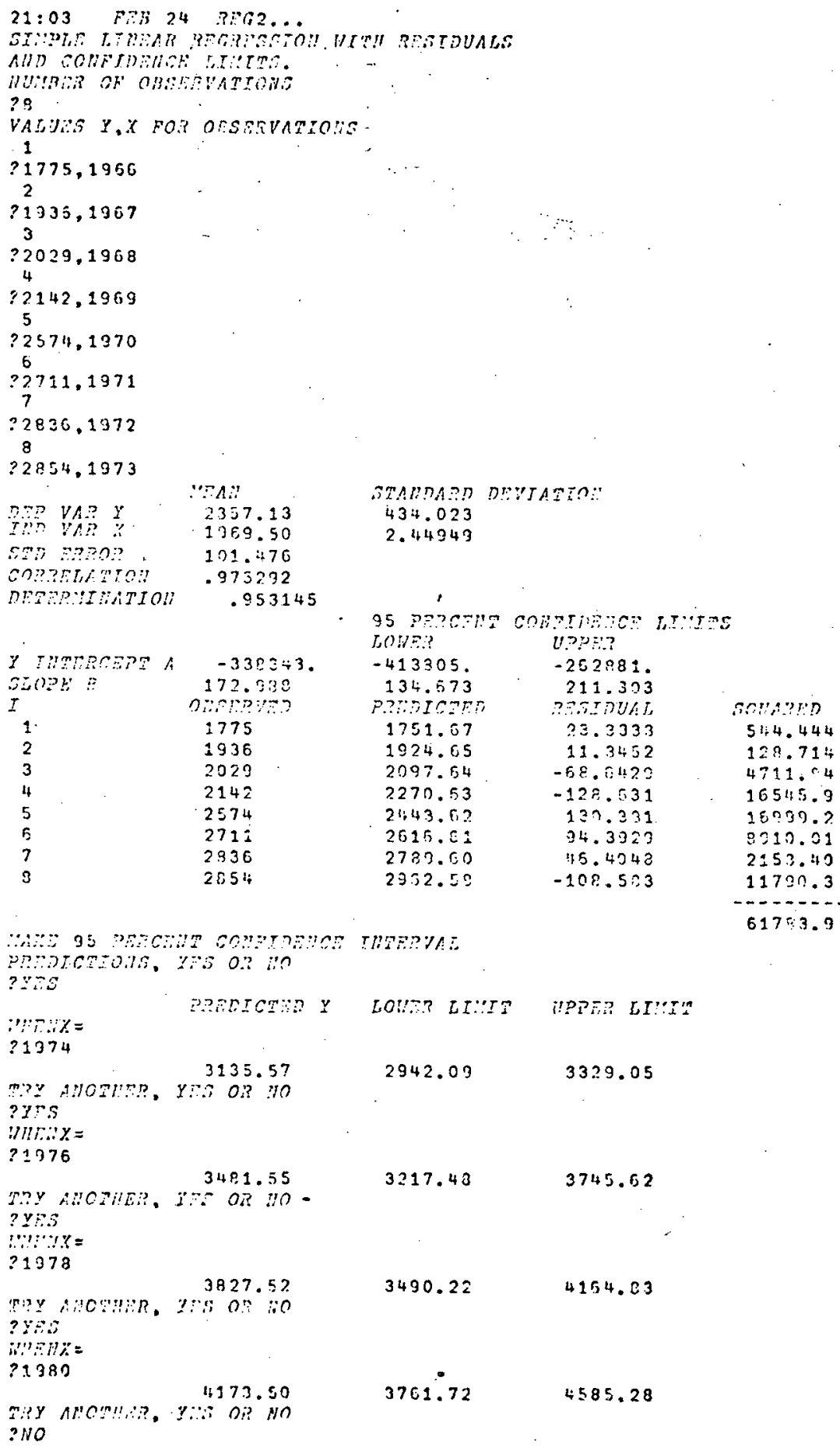

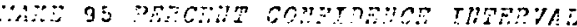

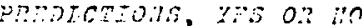

$? \because 25$

$\because \pi: x=$

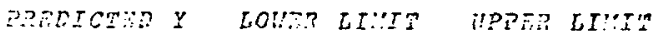

$? 1374$

3135.57

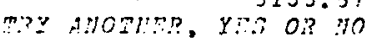

2942.09

3329.05

375

ill:.:.: $y=$

$? 1976$

$348,1.55$

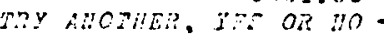

? $Y$ :SS

$\because ? \cdot \% \mathrm{Y}=$

$? 1378$

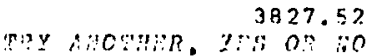

?Yiz

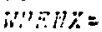

$?+380$

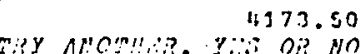

?NO

3701.72

4585.28 
TIME ANALYSIS: 8 YEAR TREND

DISTRICT \#4, RIVERSIDE-DIAS

(MTXED STRATUM)

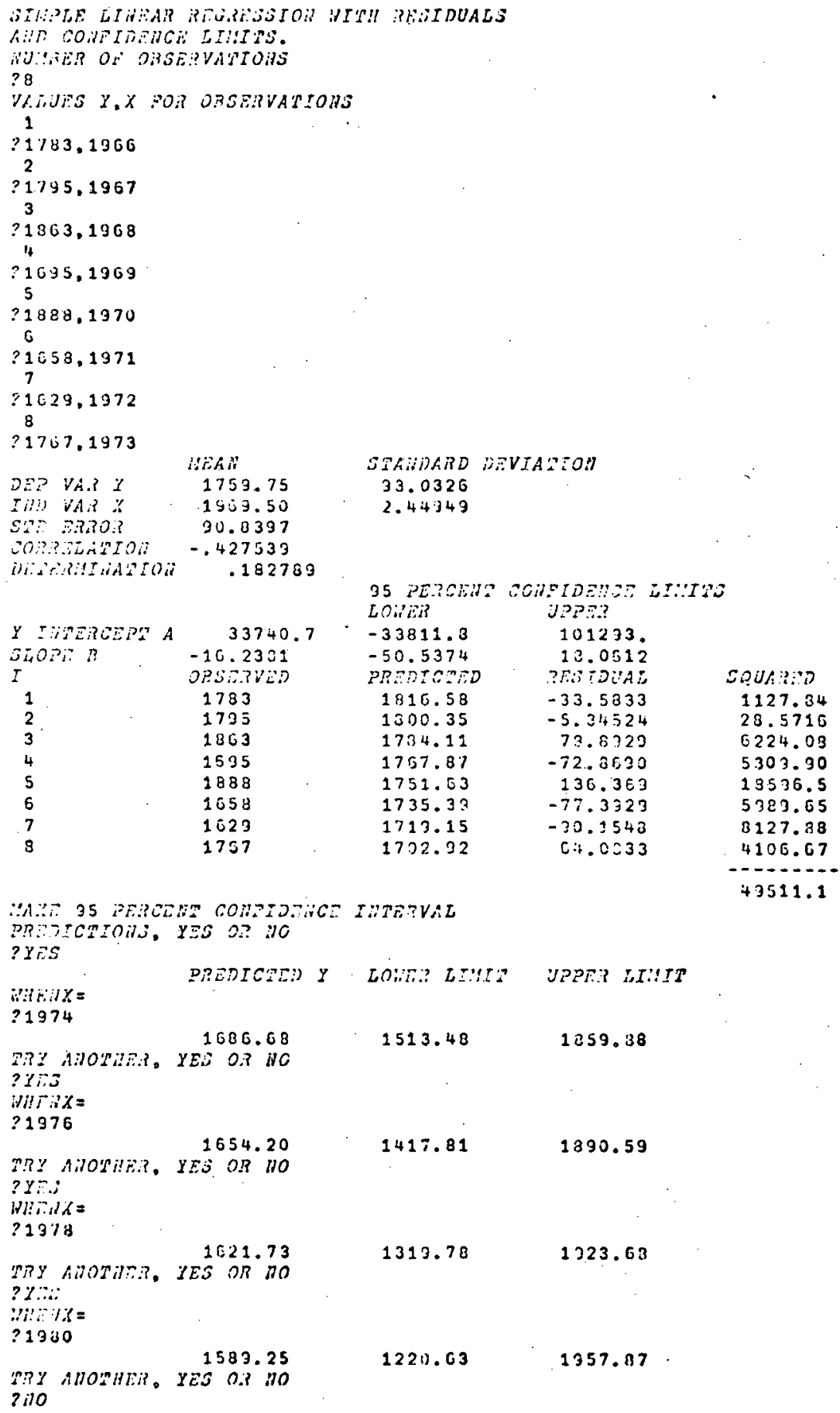


APPENDIX F2

TIME ANALYSIS

SIX YEAR TREND

SEVEN DISTRICTS 
TIME ANALYSIS: 6 YEAR TREND

DISTRICT \#9, HOPEWELL

(STRATUM \#I)

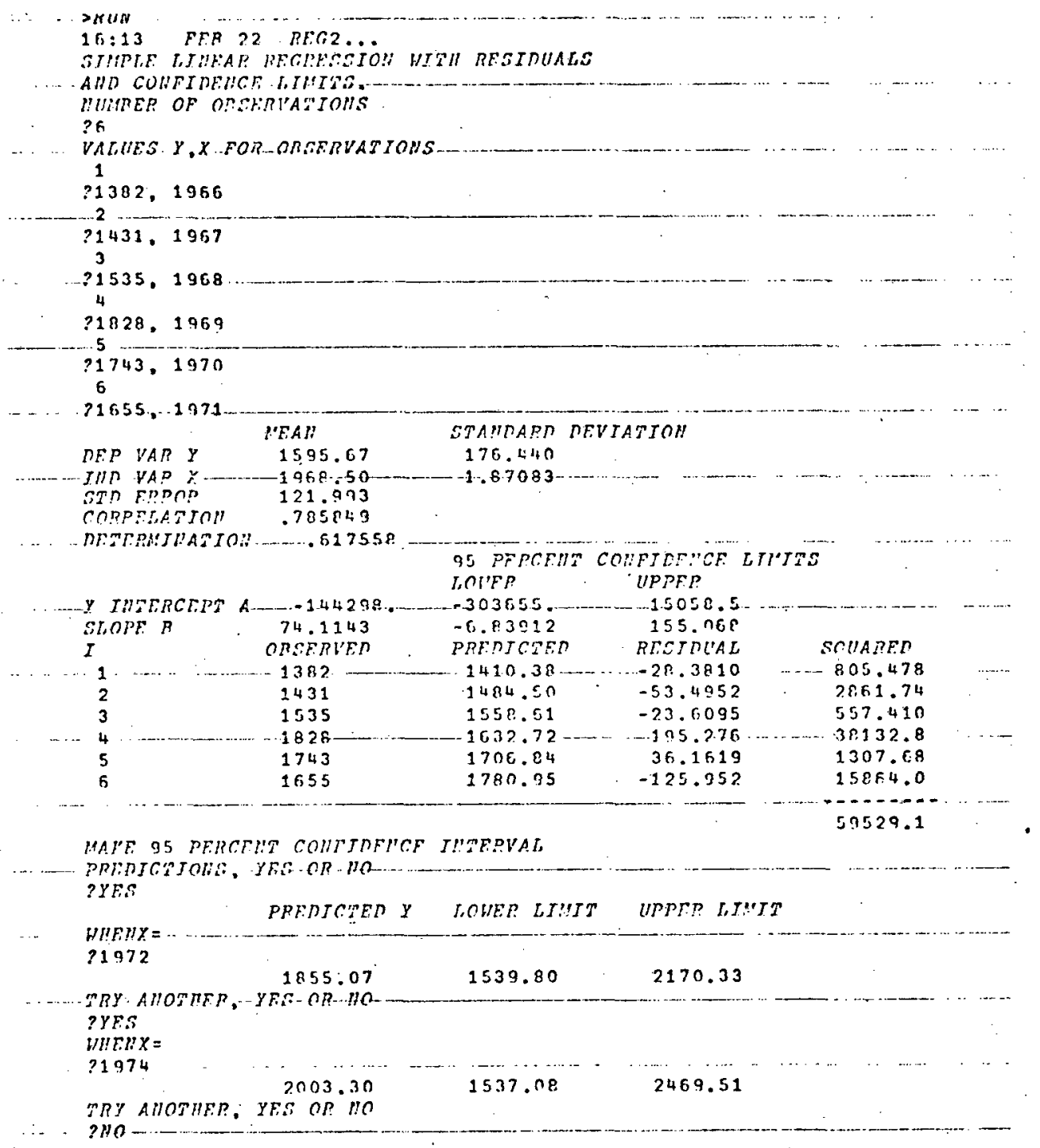


TIME ANALYSIS: 6 YEAR TREND DISTRICT \#8, SANDY BAY

(STRATUM \#II)

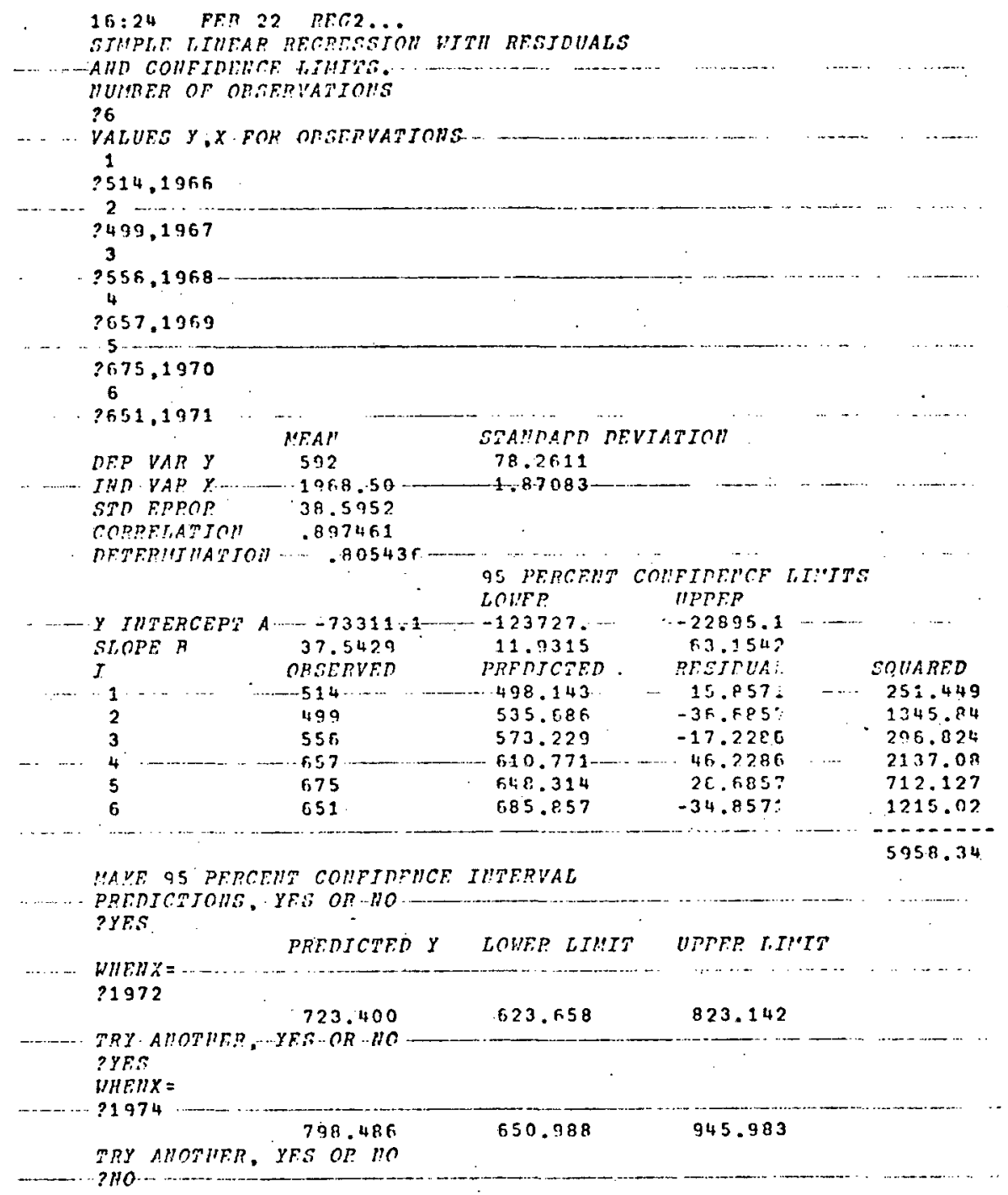


TIME ANALYSIS : 6 YEAR TREND

DISTRICT \#3, KENDAL-UPPER ROCK SPRING

(STRATUM \#III)

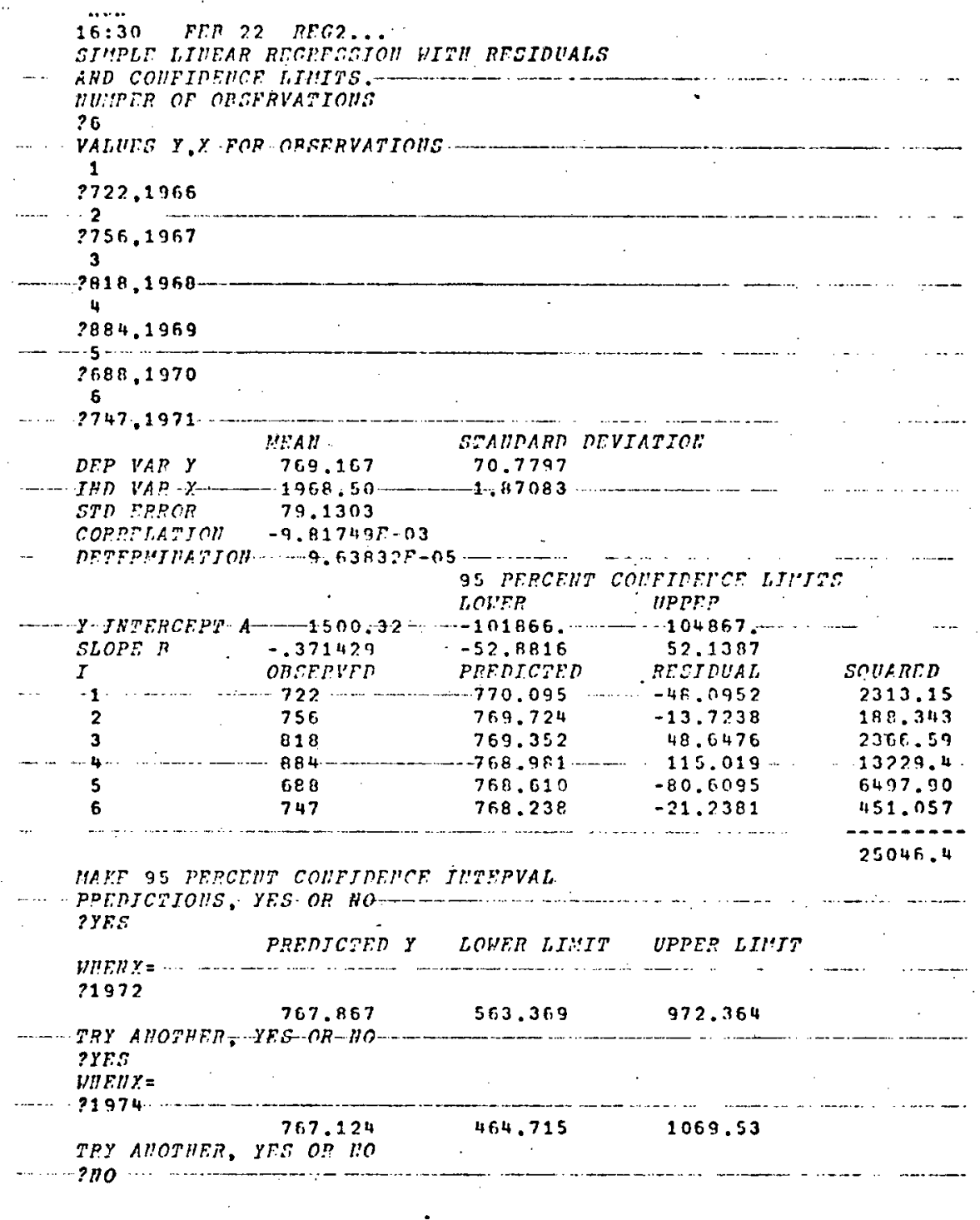


TIME ANALYSIS: 6 YEAR TREND

DISTRICT $\# 6$, JERICHO

(STRATUM \#IV)

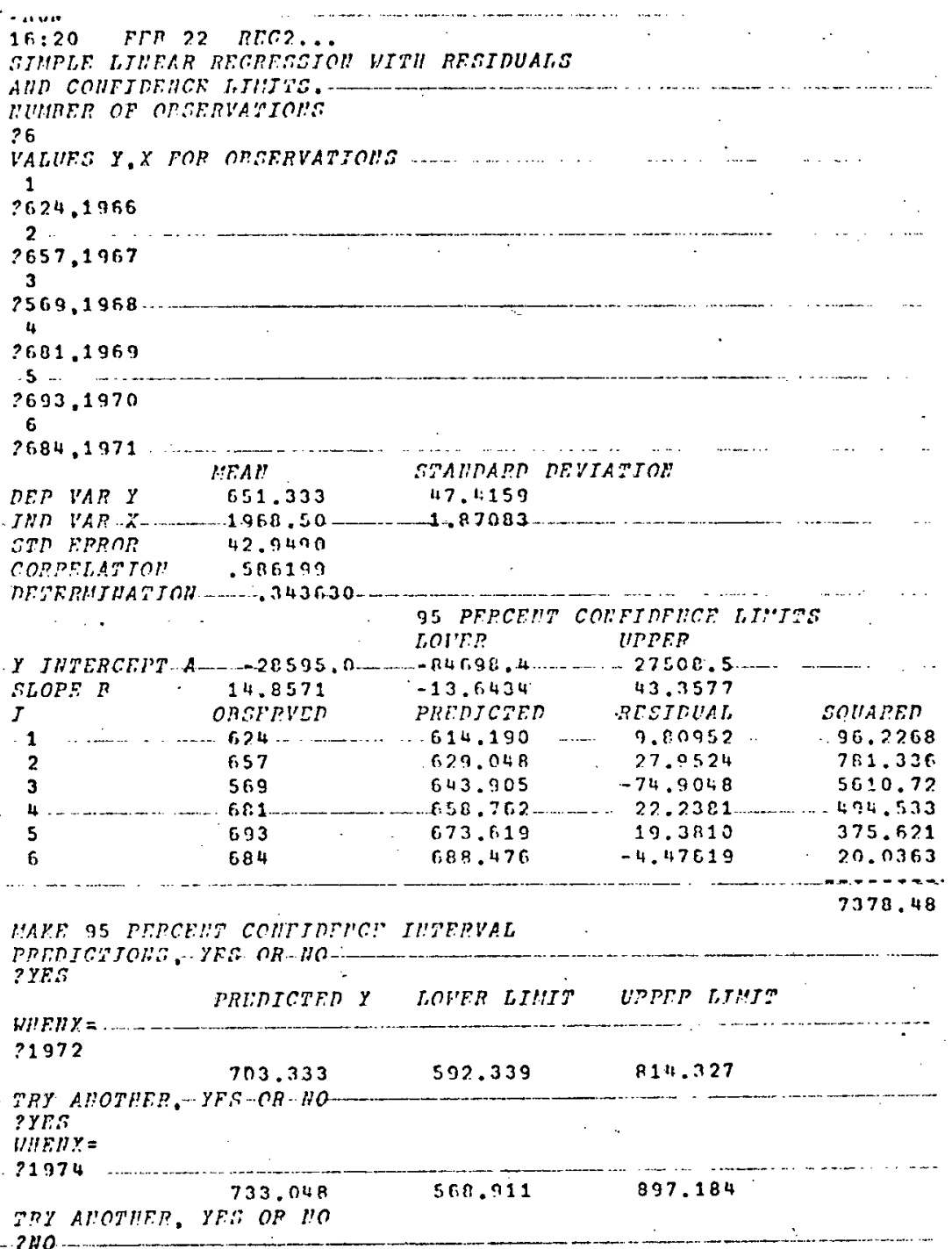


TIME ANALYSIS : 6 YEAR TREND

DISTRICT \#1, IUUCEA

(MIXED STRATUM)

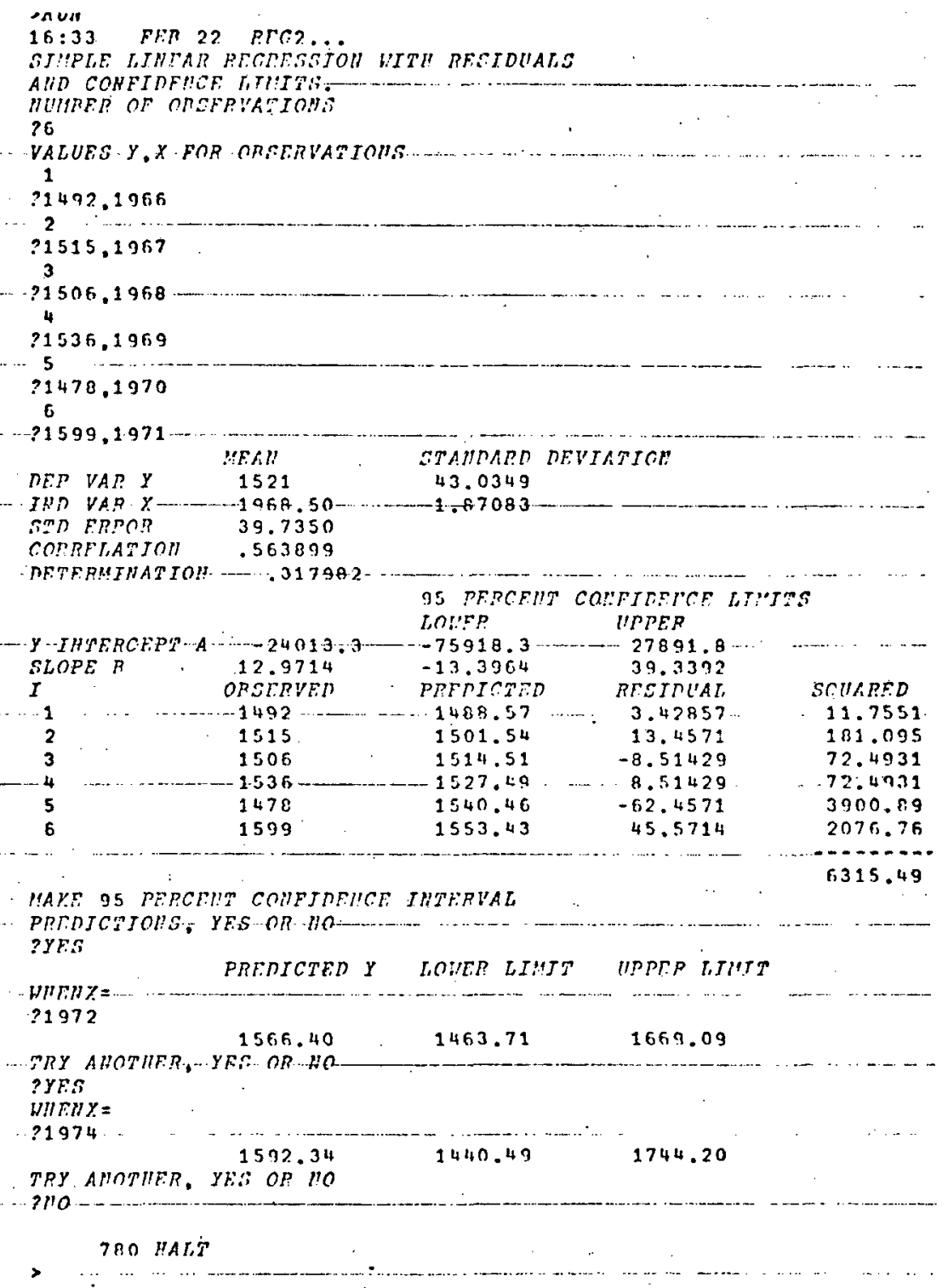


TIME ANALYSIS: 6 YEAR TREND

DISTRICT 非, GREEN ISLAND

(MIXED STRATUM)

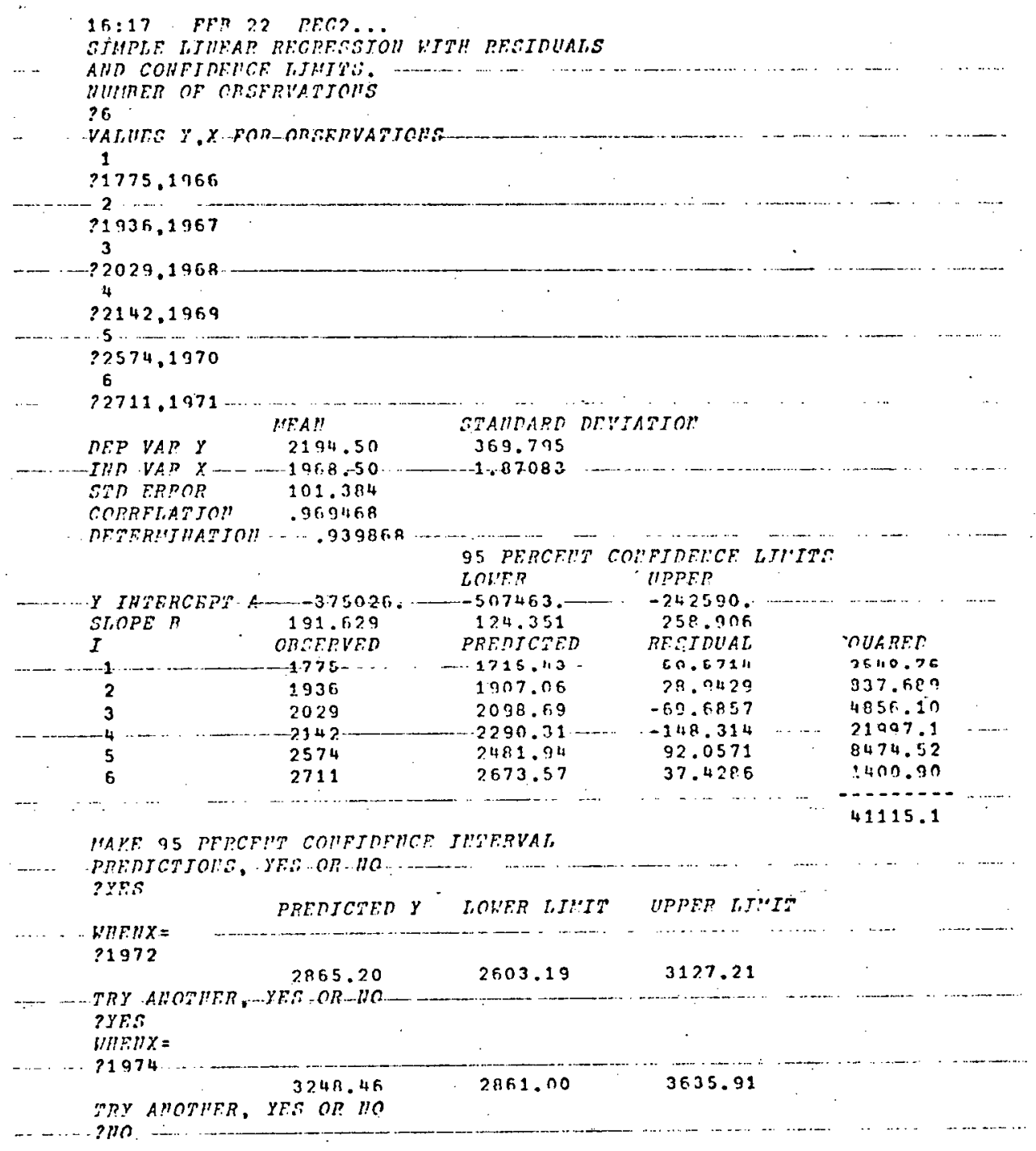


TIME ANAI,YSIS: 6 YEAR TREND

DISTRICT \#4, RIVERSIDE-DIAS

(MIXED STRATUM)

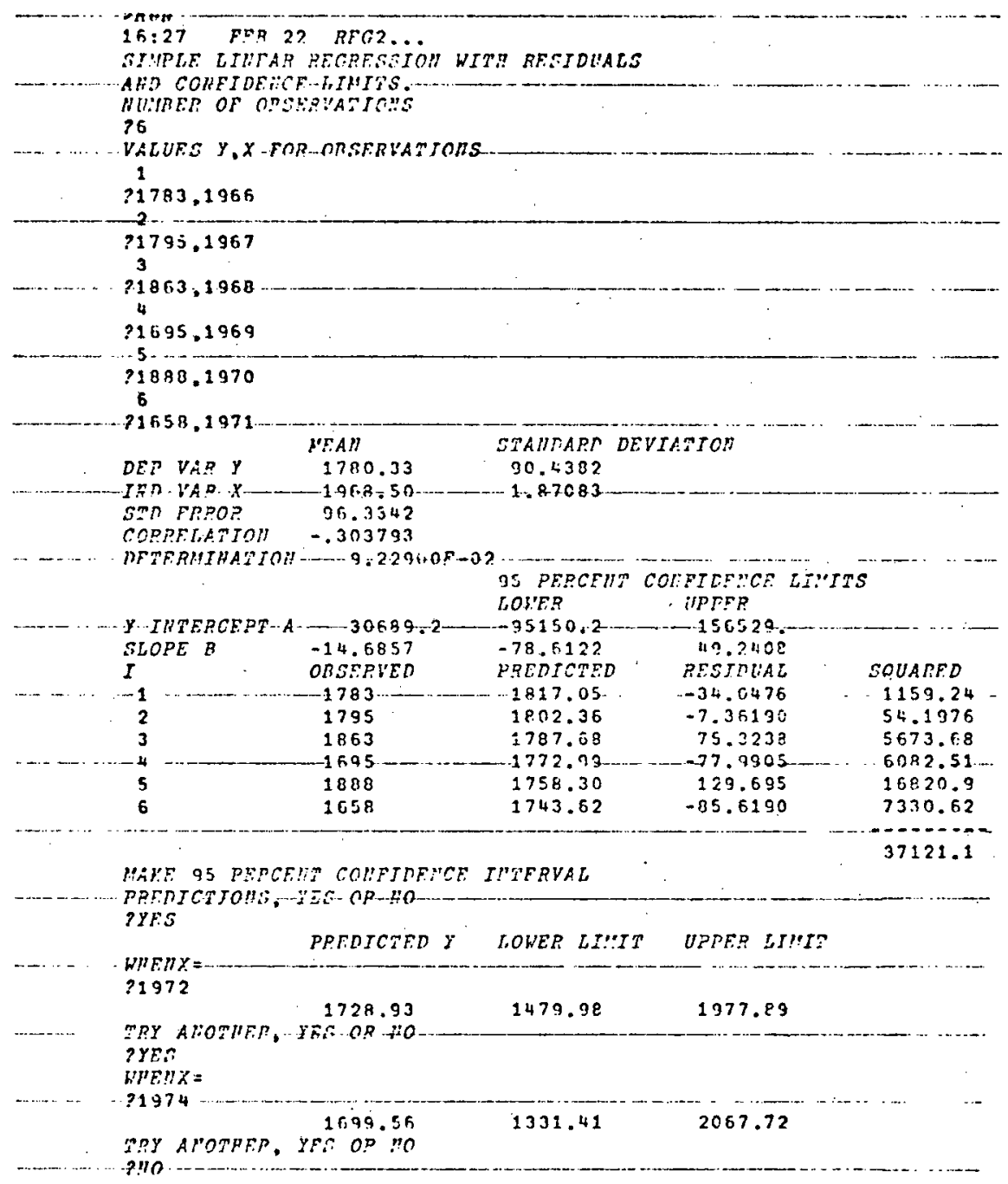


APPENDIX F3

TIME ANALYSIS

SIX YEAR AND EIGHT YEAR TREND

PARISH OF HANOVER 
TIME ANALYSIS : 8 YEAR TREND

PARISH OF HANOVER

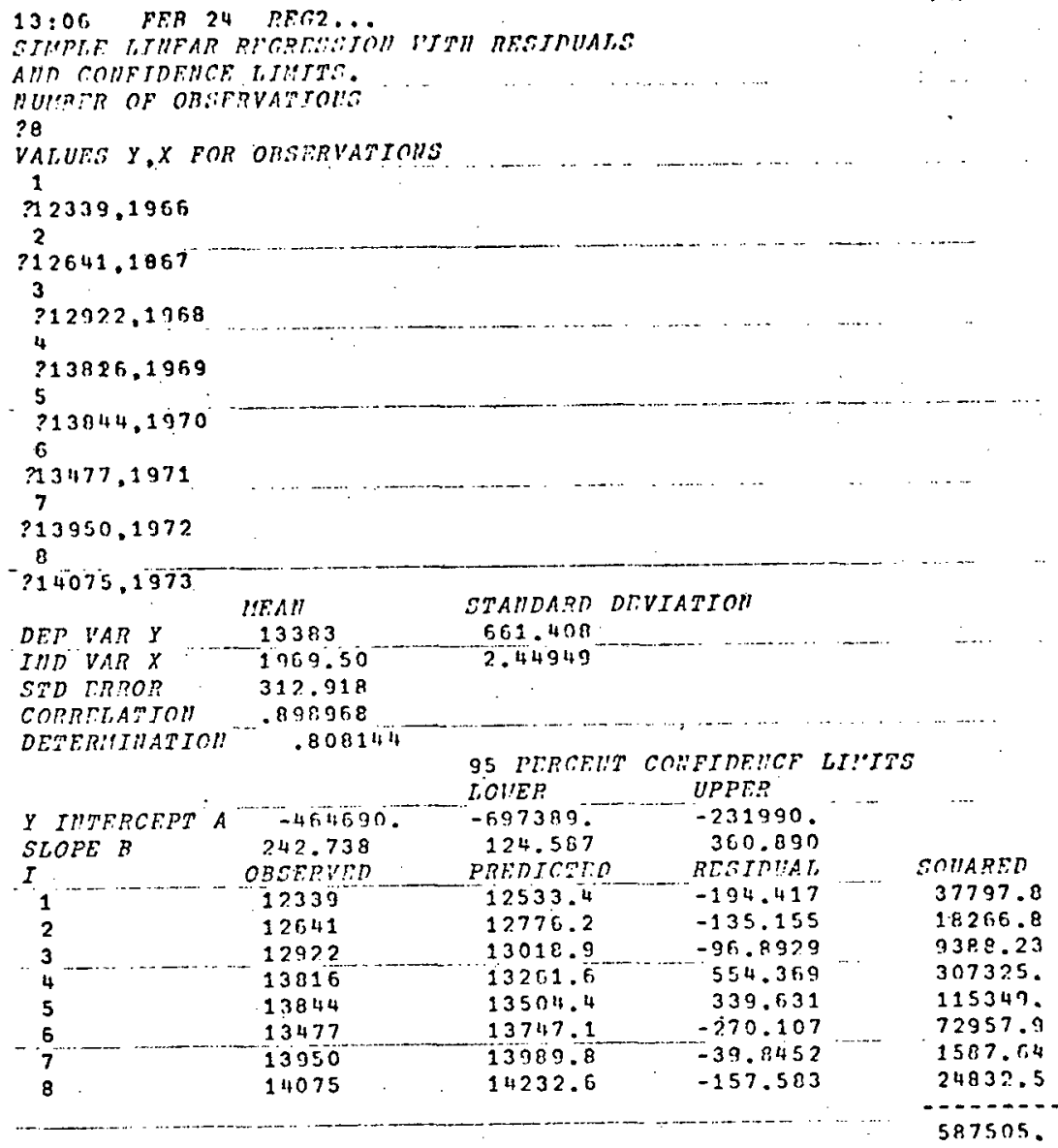

MAKF 35 PERCEIT COIFIDEICT IUTFRVAL

PREDICTIOIS, YTS OR 110

PYES

PREDTCIED Y LOIERR LILITT UPPER LIIIIT

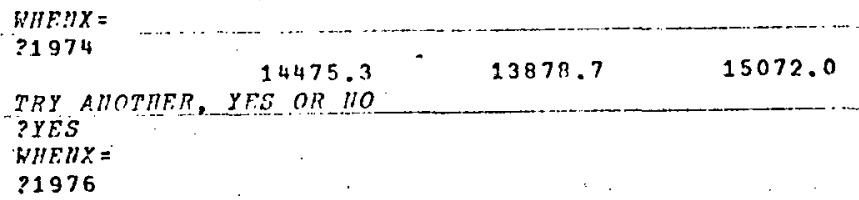




\section{TIME ANALYSIS: 6 YEAR TREND \\ PARISH OF HANOVER}

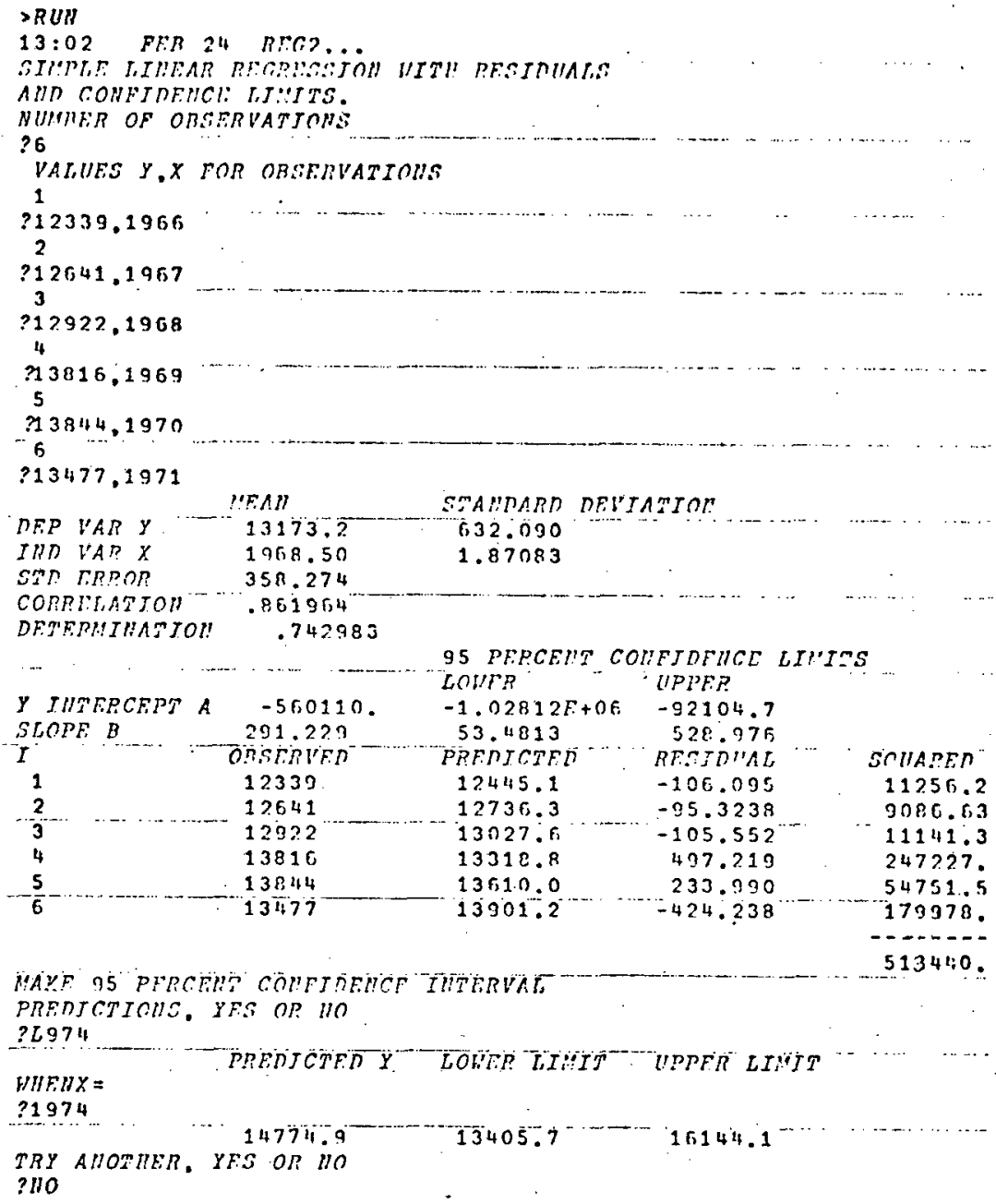




\author{
APPENDIX F4 \\ TRANS.ITION ANALYSIS \\ EIGHT - SEVEN YEAR TREND \\ SEVEN DISTRICTS
}


TRANSITION ANALYSIS: 8-7 YEAR TREND

DISTRICT 非, HOPEWELL (STRATUM 非I)

BIRTH-GRADE 1; GRADES 1-2

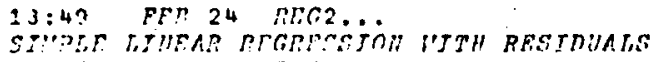

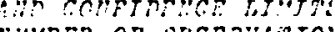

?:

1

$? 213.211$

5

2287,209

3

2307,209

212,227

5

7286,256

6

$? 253.252$

$? 299.196$

$? 273,231$

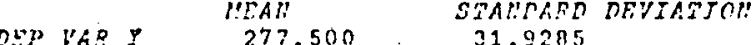

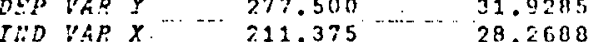
CmD EREOR 33.7934
Consträroll $\quad-.199507$

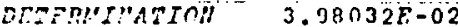

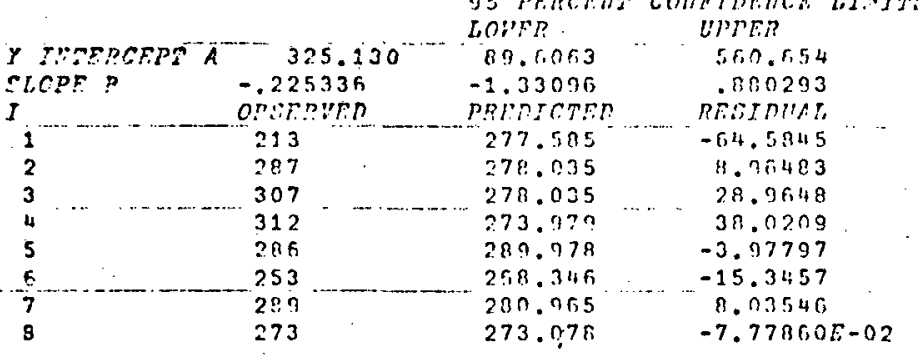

YAP O5 PERCEUT COMFIDFUCF IUTTPIAA

FPETICAIOUIS, YFS OR NQ

?QRS

PRTISCTEN Y LOWER LINIT

IPPTP LIHIT

$\because 72 \pi x=$

$276.908 \quad 247.529 \quad 306.288$

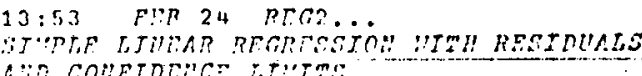

Aumere or oncenam

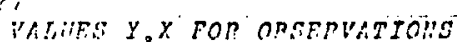

I: $: 5,213$

$? 225,287$

301,307

$2,301,307$

202,312

2244,296

2384.253

$? ? 234.253$

2277.289

DEP VAR $y$

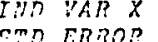

CODPRLATTOH

Domprimation

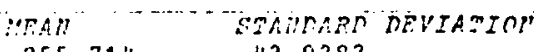

43.9383

$278.343 \quad 34.4307$

32.7415

Y TMTERCRPT A

.732306

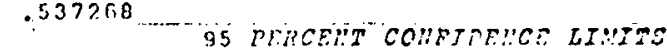

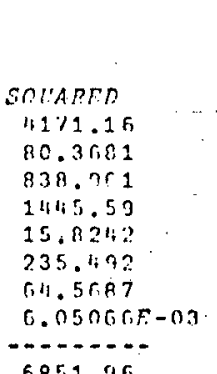

6859.96

1

2

4

?

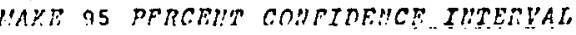

LOURP

$-11.45738$

$-283.692 \quad 274,77$

$-6.272175-02$ 1.

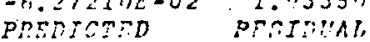

orstriven

$\begin{array}{llll}175 & 194.780 & -99.7804 & 391.254 .\end{array}$

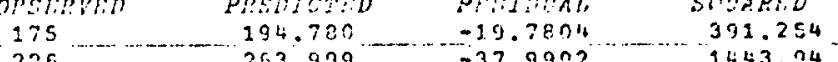

$263.399-37.0902$

SCEARED

301

301 4. 30387 1443.74
$3314,8: 36$

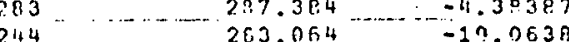
19.218 .3

263.064036327

$\begin{array}{llll}284 & 232.196 & 11.041 & 258.0 .67 \\ & 255.870 & 11.1301 & 123.879\end{array}$

5260.02

PREDSCTIOH: YES OR 110

?YRS

PRIDICTRR Y LOUER LIUTT _. UPPER LTUY?

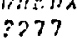

TIYY AHOTHER. YE:? OR. MO

C... 222.800

285.482

?tno 
TRANSITION ANALYSIS: 8-7 YEAR TREND

DISTRICT 非9, HOPEWELL (STRATUM 非I)

GRADES $2-3 ; 3-4$

$13: 55$ PER $24 \quad R F C 2$.

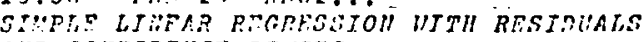

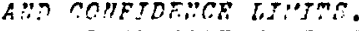

A

ALER Y,X FOR ORSTRVATIOHS

220.205

$? 210.175$

$? 214.226$

$? ?, 2,30:$

553.283

$? 245.244$

2268.284

bre $\operatorname{lin} x$

rim $\because \pi x y$

$\sin 1000,9$

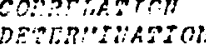

MEA 230.057 STAMDART DEVIATIOH

$233.057 \ldots 23.9056$

$21,5.1127$

$1 ; 5, .1 ; 783$

3.52065

.867823

95 neromy CONFInEMCE LIMITS LOV!?'? - UTPR

\begin{tabular}{|c|c|c|c|}
\hline \multirow{3}{*}{$\begin{array}{l}\text { Y IMATPCEPT } \\
S L O P C B \\
I\end{array}$} & & & \\
\hline & 121.263 & 67.0 .914 & 174.835 \\
\hline & $\begin{array}{r}479135 \\
\text { OREFRER }\end{array}$ & 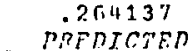 & .694136 \\
\hline 1 & 220 & 219.186 & $.513 r, 55$ \\
\hline 2 & 210 & 205.112 & 4.88775 \\
\hline 3 & 211 & 229.548 & -15.5482 \\
\hline 4 & 252 & $26,5 . t, 83$ & -3.483113 \\
\hline 5 & 253 & 256.853 & -3.05898 \\
\hline 6 & 245 & 238.173 & 6.82734 \\
\hline 7 & 268 & $257.33 R$ & 10.6619 \\
\hline
\end{tabular}

NARE 95 PERCENT COMFTDERCE IURRRVAR

PPEATCTICHS, YE: OR NO

$$
\text { PYE. }
$$

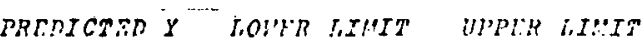

:yry $=$

$2255-243.443$

233.965

252.921

TRY ANOTHEP. YTS OP 10 ?310

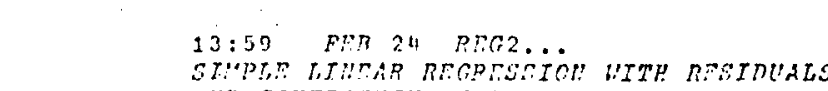

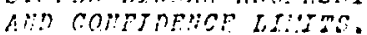

minor of oscirvaryons

$? 158.196$

2

214.220

$? 201.210$

$? 223.224$

??32.26?

6

2142,253

$? 255,245$

DIP VAR $Y$

IPD I'AR K

ITD RRPOR

DE'TERTHATOU

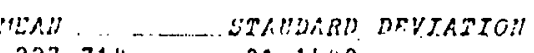

$227.714 \quad 31.1540$ 211.7212

29.3151

5350141

$.2756 r_{18}$

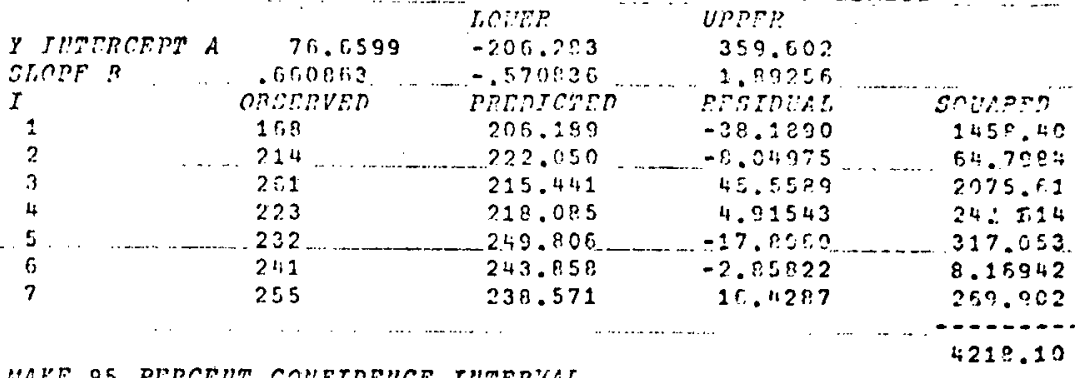

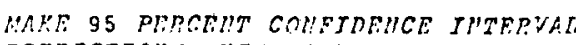

BRFDSCITCLI: YTS OR :O

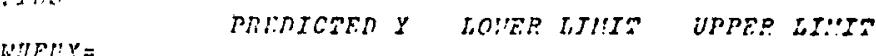

$? 243$

237.250
MRY ANOTHER, YEE OR 110

203.830

270.603

$\therefore 0$ 
TRANSITION ANALYSIS: 8-7 YEAR TREND

DISTRICT 非, HOPEWELL (STRATUM 非I)

GRADES $4-5 ; 5-6$

14:02 FER 24 RTC2,

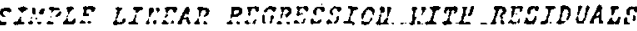

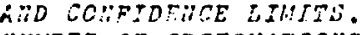

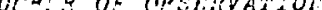

??

LUFS Y,X FOR ORPERVATIONS

$2.257,243$

2

$?: 51,158$

211.214

$? 191.261$

$? 179.223$

?194:.232

7

$? 211.241$

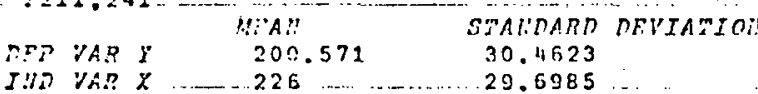

STD ERROR 27.9075

COPPELATIOI .54,825

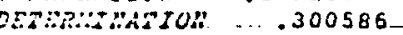

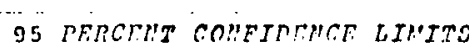
LOUIR R UPRTR

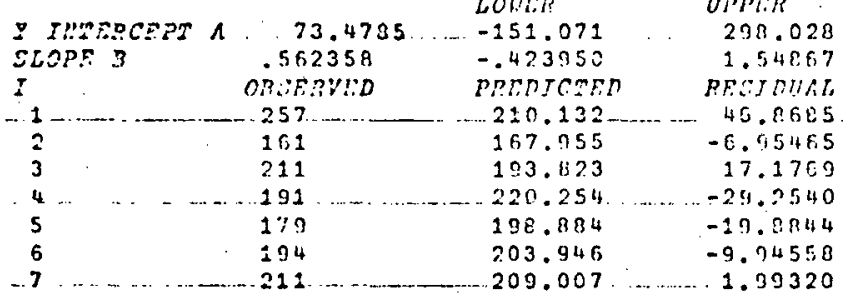

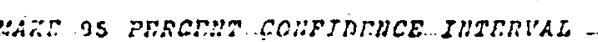

TENS OR 110

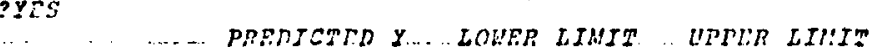

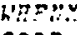

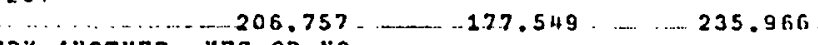

MPX AHOTHER, YES OR VOO

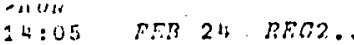

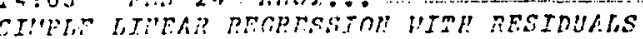

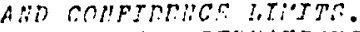

$? 7$ THES Y Y POR CRSERVAMTOHS

$? 117,130$

$? 36,257$

$? 130.161$

$? 1014,211$

$? 103,131$

$\because 133,179$

$? 113,194$

DER VAR Y

IHID VAR $X$

Sim GRPOR

CONDEEATISH

DE'SER:'IIIATJOL

UTAl STANDAPD DFVTATIOH

26.3281 
TRANSITION ANALYSIS: 8-7 YEAR TREND

DISTRICT \#9, HOPEWELL (STRATUM \#I)

GRADES $6-7 ; 7-8$

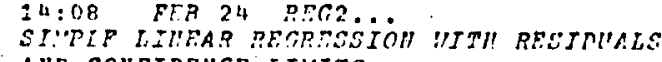

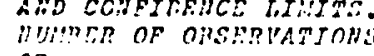

?7

"HEUES $Y, X$ for OHSERVATIOHE

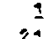

1219.12

$? 2$

3

4

$\because 254,130$

5

$? 164.104$

7

?.

$? 297.133$

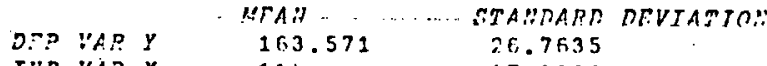

Jin VAR Y $Y$ 114

ST) TP?OR

CONPRLATINI $\quad-.105320$

OETER!'THATIOI $\quad 1.10924 E-02$

\section{C... 95 PRPCEWT COHFIDFHCE BIIITS}

YITSRCEPT A $182.376 \quad-73.7312$

SLOPR 3 .....

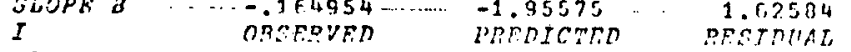

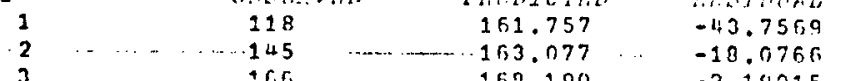

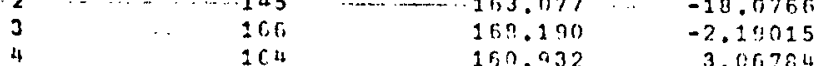

$\begin{array}{rrrr}164 & 150.932 & 3.06784 \\ 5 & 164 & 165.221 & -1.22097 \\ 191 & 165.326 & 25.6149\end{array}$

$\begin{array}{llll}6 & 191 & 165.386 & 25.6141 \\ 7 & 197 & 160.437 & 36.5627\end{array}$

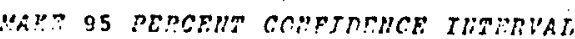

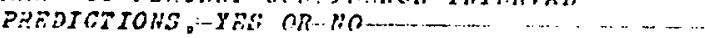

?.F?

WREHA

PREDICTED Y . LONER LIIITT UPPLR LIS:IT

$2: 07$.

SPY AMOFHER, YFS OR 16,726 $\begin{array}{ccc}164.726 & 133.746 & 195.707\end{array}$

$\begin{array}{ccc}164.726 & 133.746 & 195.707\end{array}$

(1)..................

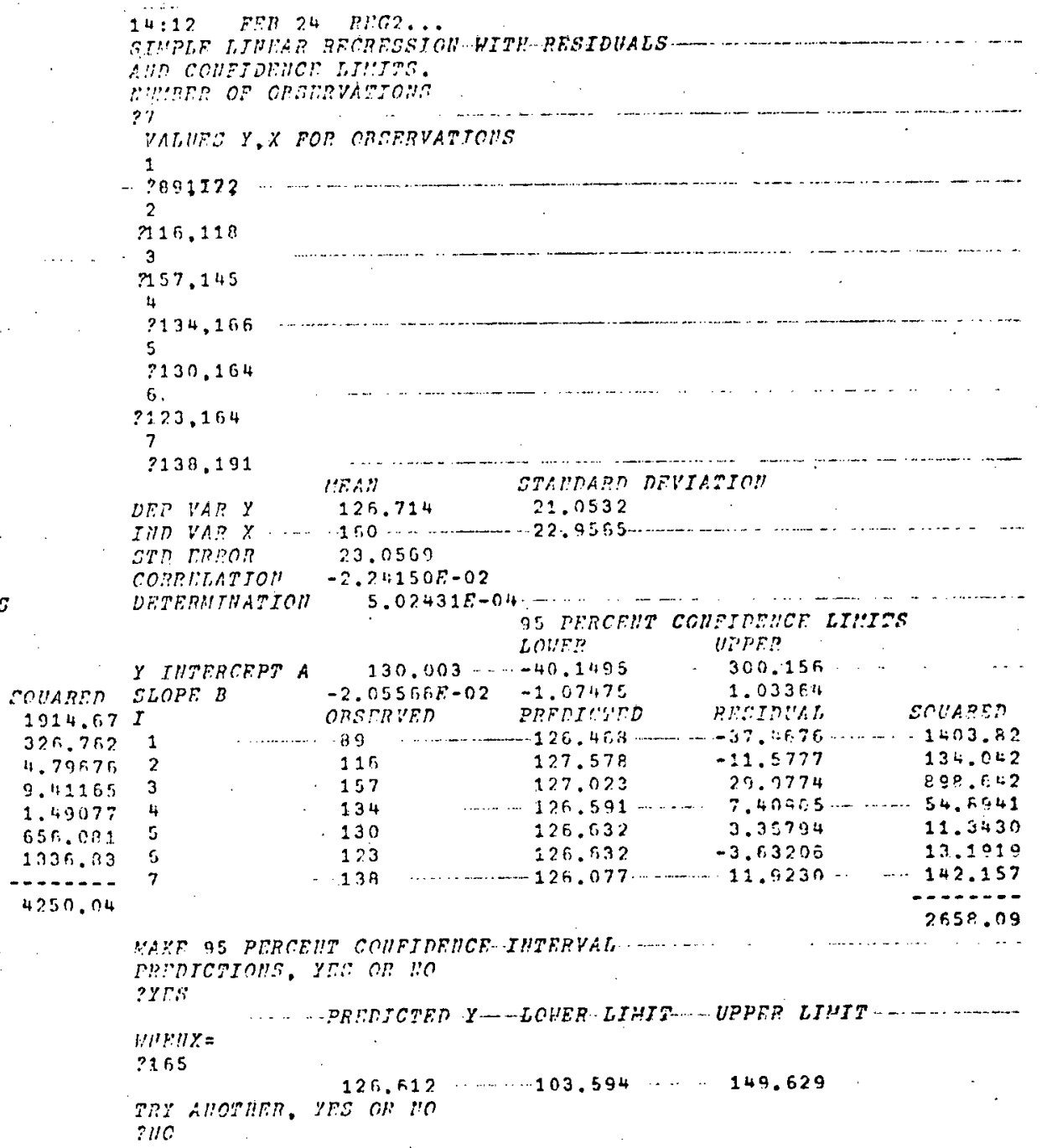

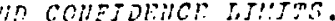

1

715.118

2157,145

$? 134,166$

?130.164

$? 223,164$

7

UTA

126.714

STAVARA REVIATIO!

TRY AlOAHER, YRS OR IIO

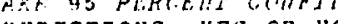


TRANSITION ANALYSIS: 8-7 YEAR TREND

DISTRICT \#作, HOPEWELL (STRATUM 非I)

GRADES 8-9

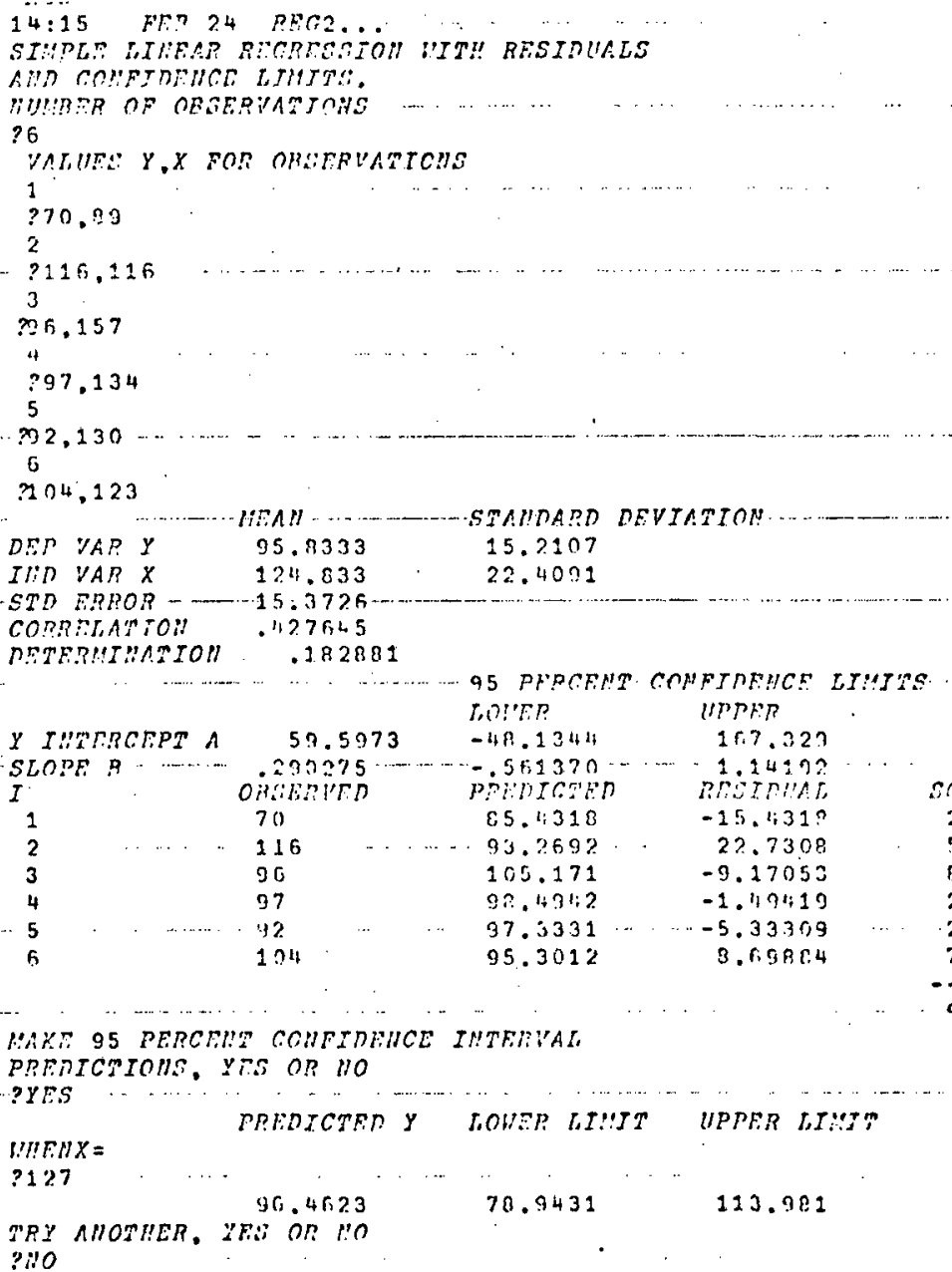


TRANSITION ANALYSIS: 8-7 YEAR TREND

DISTRICT \#8, SANDY BAY (STRATUM 非II)

BIRTH-GRADE 1; GRADES 1-2

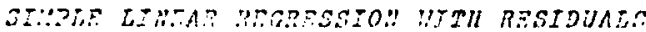

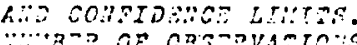

?

ILES $\%$ Y FOR ORSE?MATIOHS

$? 24,108$

2

? 614.105

3

2108.113

$? \begin{aligned} & 4 \\ & : 126,34\end{aligned}$

$? 122,91$

$\because 202,83$

792,8

2120,122

\begin{tabular}{|c|c|}
\hline & \\
\hline$\therefore \triangle P$ VAR Y & 107 \\
\hline 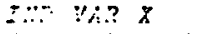 & 105 \\
\hline$\because \quad \because 20 \Omega$ & 24.7834 \\
\hline 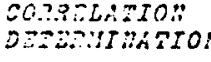 & $\begin{aligned} 2 . & ? 0574 \pi-02 \\
& \quad .32104 F-0\end{aligned}$ \\
\hline IUTRSCEP & 102.750 \\
\hline $02: 3$ & $\begin{array}{l}5.00000 \pi-02 \\
0 \text { क.REYRD }\end{array}$ \\
\hline & 84 \\
\hline 2 & 64 \\
\hline & 108 \\
\hline & 226 \\
\hline & 122 \\
\hline & 102 \\
\hline & 130 \\
\hline & 120 \\
\hline
\end{tabular}

STANDSPD DRVTATION

22.9534

12. i 212

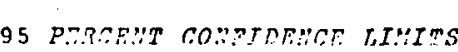

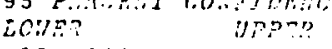

$\begin{array}{ll}-93.1963 & 296.696\end{array}$

$-1.79537$

prentCren

107.150

107.250

107.400

$106.1: 50$

106.300

136.200

107.0120

137.850

RE.TRY:AL

$-23.9500$

$-43.19500$

.600000

19.5500

15.7000

$-4.20000$

12.1500

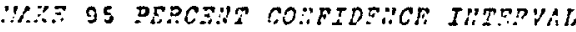
PSEDTCRTONS. YES O? 20

$3=2=3$

PREDTCTRD Y EOHER LTUTT UPPFR LITITT $: \because: 3 x=$

$107 \quad 85.5588 \quad 128.441$

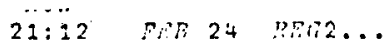

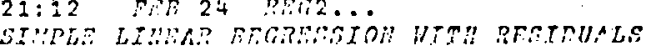

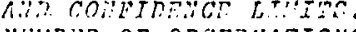

$? 7$

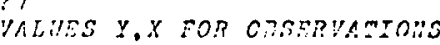

237,84

2

271.64

3

$? 20.108$

$: 105.126$

3

$? 142,122$

$? 97,102$

$? 126,130$

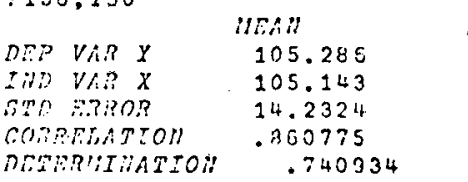

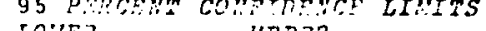

$\begin{array}{llll}y \text { INTRERP A } & 9.56377 & -50.3699 & 75.0965\end{array}$

$\begin{array}{lrlll}3 L O P E & 0910393 & .231437 & 1.52935\end{array}$

RFITINAL

$36.0373 \quad 957721$

8.8 .2 .3

107.837

$12 \div .274$

-10.2740
21.3676

-5.424 ir

8.03437

240.490

$50 \AA .760$

$24 \% .622$

3605 30 :AME 95 PERCWM CONRIDEMCR IUTERYAL

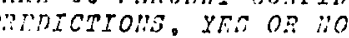

?y:

$\ln \Gamma: x=$

BRINTER $y$

LOWH? LII:T

UPDET LI:UTT

$? 107$

a?Y AMOMER, YRE 9897

93.0985

120.854
5014:50=

$0.02583:$

10.0333

73.3762

$37: .428$

458.573

23.4248

65.3570

1012.80 
TRANSITION ANALYSIS: 8-7 YEAR TREND

DISTRICT 非, SANDY BAY (STRATUM 非I)

GRADES 2-3; 3-4

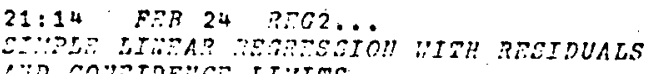

$21: 17 \quad P R 2 \quad 24 \quad \operatorname{Rrg} 2 \ldots$

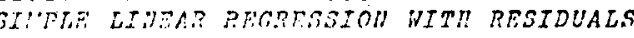

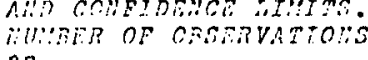

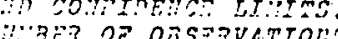

27

YhLUES Y, $X$ PO? ORSERVATIOUS

$$
\begin{aligned}
& 1 \\
& ? 96.69 \\
& 2 \\
& ? 99,87 \\
& 3 \\
& ? 77,71 \\
& 4 \\
& ? 93,93 \\
& 5 \\
& ?: 21,105 \\
& 5 \\
& ? 133,142 \\
& 7 \\
& ? 50.97
\end{aligned}
$$

\begin{tabular}{|c|c|}
\hline Y.ZTRPCEPT & 25.4727 \\
\hline 210032 & .724912 \\
\hline & $0 \operatorname{sen} 48 \pi$ \\
\hline 1 & 96 \\
\hline 2 & 89 \\
\hline 3 & 77 \\
\hline 4 & 93 \\
\hline 5 & 111 \\
\hline 6 & 133 \\
\hline & 35 \\
\hline
\end{tabular}

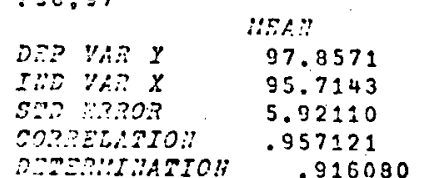

STAEDABD DEVIATTOH

18.6596

$24.5,354$

CORDELTYOH .957121

95 PERCENT CONPIDPHCR LIUTTE LOWE? uppe?

$3.65064 \quad 5.3 .2948$

$1172640 \quad 977404$

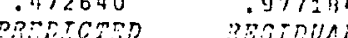

$78.4916 \quad 7.50936$

$31.5401-2.54005$

$79.9415--2.94147$

$100.239-7.23000$

104.588

$131.413 \quad 6.53930$

38.7832

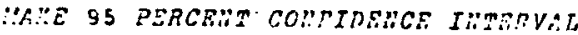

PDEDECTIOHS, YRE OR HO

? $2: 25$

PRENTCTED Y LOWRR LIIITT UPPER RIITT

$n 112 \pi x=$
$? 107$

106.038

m?Z AMONHER, YRS 0.? 110

99.6186

112.458

?.10

9.61060

?79.134

255,

$?: \pm 8,89$

273.77

275.93

6

$? 101,111$

$? 122.133$

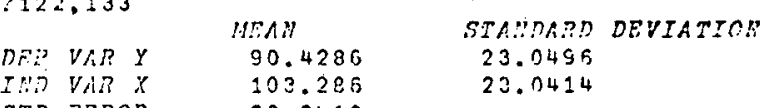

22.2410

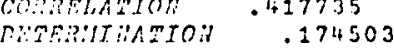

95 PERCRHT CONIDENCE LIUISS LClip?

$-1,2.3482 \quad 157.452$

$-527150 \quad 1.46292$

PSTRTCED
103.264

$\begin{array}{ll}102.264 & -24.2635 \\ 53.2051 & -18.2051\end{array}$

S3.2051 $\quad-18.2051$

73.4442

90.1303

93.6523

3.3 .5412
-5.4 .1417

6.45183

8.6522 .3
52.4031

41.1073

2.52746

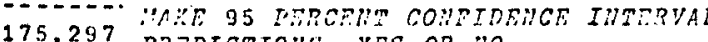

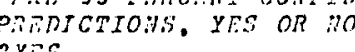

$?$ \&is

$-11.1303$

7.34774
19.1543

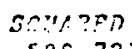

505.723

$312.22 ?$

1125.51

$4: 25273$

53.9893

366.885

365.886
-

2531.45

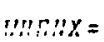

2100

206
$? \because \because 0$

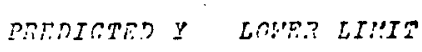

".pos? 2.T.T2

63.0902

114.035 
TRANSITION ANALYSIS: 8-7 YEAR TREND

DISTRICT 非, SANDY BAY (STRATUM 非I)

\section{GRADES $4-5 ; 5-6$}

$24: 20$ FEB 24 Praz

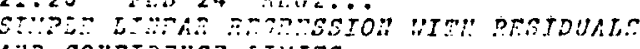

4.2.

? 7

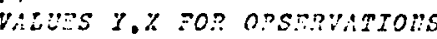

$? 56.57$

$? 35.79$

$? 31.55$

$\stackrel{4}{? 72,1 \pm 8}$

$? 72,118$

245.73

$? 73.75$

$? 82,101$

\begin{tabular}{|c|c|}
\hline & $\because \pi A i$ \\
\hline$\cap E S$ VAS Y & 67.8571 \\
\hline 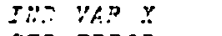 & 31.1429 \\
\hline STS PRTOA & 14.3605 \\
\hline $0 \Omega 8=28: 00 \%$ & .1147346 \\
\hline ENERUTISATOOH & .200560 \\
\hline IUTEPCRRT A & 41.8738 \\
\hline $\begin{array}{l}S L O F E B \\
I\end{array}$ & .320218 \\
\hline & 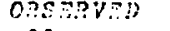 \\
\hline 1 & 65 \\
\hline & 25 \\
\hline 3 & 51 \\
\hline & 72 \\
\hline & 45 \\
\hline & 73 \\
\hline & 82 \\
\hline
\end{tabular}

STAHDARD DEVIATION

15.1814

21.232

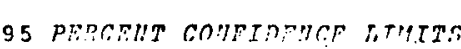

LOU:?

$-13.4306$

$-.414946$

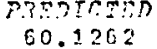

60.1262

67.1710

62.0 .873

3.6594

65.2479

05.8901
74.2157

y?p?:?

103.244

1. 25528

$\operatorname{sen}$
5.0739

13.8290

$-11.6279$

$-7.05345$

$-20.2 .497$

7.10991

7.78425

MY 95 PORCEIS COUFIDFHCE IUTERVAL

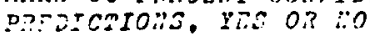

?YES

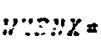

PAPDCTER $y$

LOMT? LIHIT

UPtiR LT:IIT

$? 92$

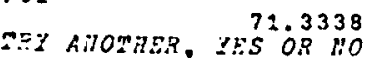

54.8270

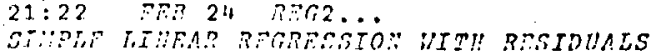

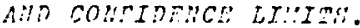

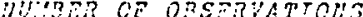

$? 7$

VALIUTS Y, X FOR ORSRPVATIOUS

? 52.28

20

$26: .05$

$? 41,80$

433,51

5

? 36, 72

6
$? 29,45$

$? 29,45$

$? 30,73$

OPR VIR

IIEAL:

TTIITART DEVIATIO:

smb

40.2857

12.0515

60.1429

15.9110

CORs?LATIOA -.127240

DESRR:THATON $1.61323 \mathrm{~F}-02$

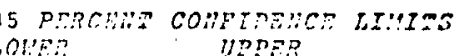

Y TITERCEDT A

95

SLOP!: $: \quad \begin{array}{ll}44.3413 \\ \text { I }\end{array}$

$\therefore .32378$

$\begin{array}{ll} & -.731973\end{array}$ PDENIC:R.

42.7738

33.8323

33.2942

$34.501 \%$

354.533.

136.607

58.6572

410.049

$50.550 \%$

60.5946

1105.5

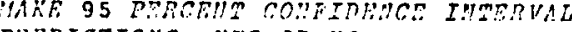

33.3570

41.4579

33.2905

89,5628

98.5628
.515345

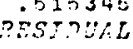

$3.225: 5$

21,1577

$-7.09345$

$-3.36737$

$-12.4573$

$-9.29046$

Gล:

85.1220

440.771

53.895

11.3425

155.199

86.3127

857.318

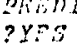

Wilitir $X=$

$? 71$

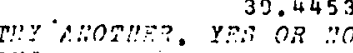

?:10

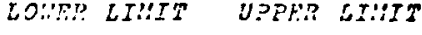

24.6590

54.2319 
TRANSITION ANALYSIS: 8-7 YEAR TREND

DISTRICT 非, SANDY BAY (STRATUM \#II)

GRADES $6-7 ; 7-8$

$21: 25 \quad \operatorname{sen} 24 \quad \operatorname{Dec} 2 \ldots$

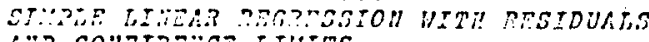

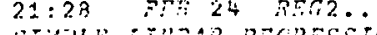

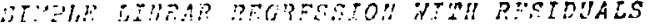

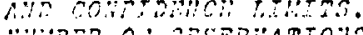

?

a.

.7

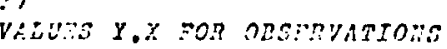

1

31.47

$? 50,52$

$? 50,52$

$? 63,61$

$? 83.41$

$? 83.41$

$? 56.33$

$? 70.35$

$.7 \%$

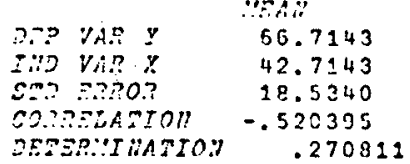

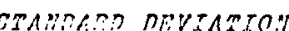

19.8134

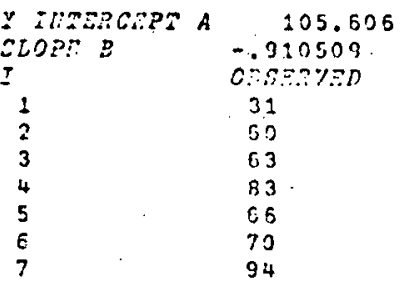

95 PERCRHA CONFIRANCE LRUITS

$30.0508 \quad 131.101$

$-2.522037 \quad .307352$

02.3121

$52.3121 \quad-31.8121$

$\begin{array}{ll}50.2595 & 1.74744 \\ 50.0650 & 12.9350\end{array}$

$68.2752 \quad 14.7240$

$75.5532 \quad-3.55923$

$72.8277 \quad-2.82770$

$79.2013 \quad 14.7987$

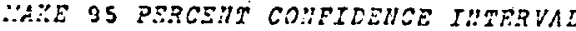
PRADICTIOAS. YES OR UO

?Xes

PREDICEES Y LOIRR LIUIT UPRER LIUIT $\because \because: \therefore i \pi \ddot{i}=$

70.0962

T?: AHOTHER, YFS OP 70

50.9889

89.2034

\begin{tabular}{|c|c|c|}
\hline & $\begin{array}{l}\text { Y IITERCESZ A } \\
\text { SLOLE } R \\
I\end{array}$ & $\begin{array}{l}47.0134 \\
-.101364 \\
0 D S \operatorname{IDVSD}\end{array}$ \\
\hline & 1 & 34 \\
\hline RUYART? & 2 & 47 \\
\hline 10.22 .01 & 3 & $5 ?$ \\
\hline 3.02913 & 4 & 36 \\
\hline 167.315 & 5 & 47 \\
\hline 216.821 & $b_{5}$ & 31 \\
\hline 34.3783 & 7 & 38. \\
\hline
\end{tabular}

GTAMDAR DEVIATOR

$? 34.62$

2

$? 47,31$

$? 52.60$

$? 36.63$

$?+4.83$

? 31.66

7

$? 38,70$

DUi VAR

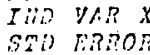

:ran

7.91021
15.7420

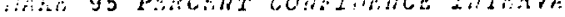
95 PERCEIIT CONPERENCE LI:ITES DERTZZTHATEOH $4.00323 E-02$

10.2337

10.2337
-.067245

PinnICAT

40.7288

43.3711
40.9315

40.9315
40.5274

40.6274

38.6001

40.3233

39.9179 UPPE:
83.1330
. 454517
RECrTis:
$-5.7387$

3.12894

11.0685

$-4.62710$

8.39088

-9.32331
-2.31785

SEva?t:

45.2753

3.73025

222.512

21.4120

$70.558:$
$85.924:$

$85.924:$

$3.678: 5$

$3 \in 0.252$

?YFS

IIII $=$

270

39.9179
TRY.AHOTHR, YRS OR :10
? WO

LQUT: LI:!IT

JPPER LI:AT

30.5484

49.2873 
TRANSITION ANALYSIS: 8-7 YEAR TREND

DISTRICT \#8, SANDY BAY (STRATUM \#II)

GRADES 8-9

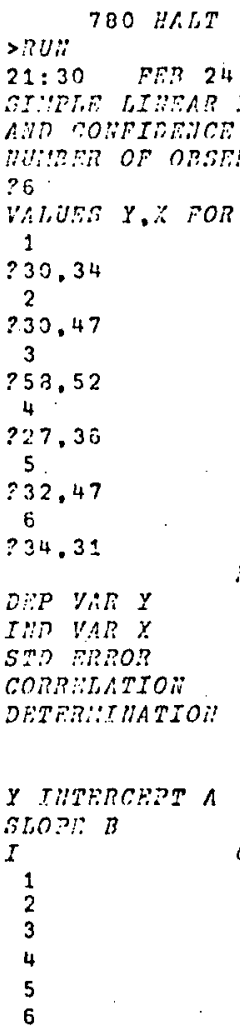

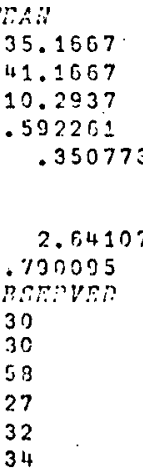

STABZAMT DEVISIO

11.4206
8.505113

35 PSRCEHT COHETDRMCE LIMITS

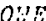

$-53.8 .757$

.701856

23.5043

33.7750

43.7260

31.0845

33.7755

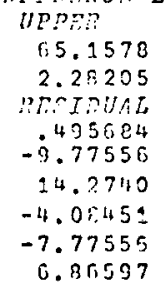

SOYURE.

2145702
35.5615

203.746

15.0532

50.4503

47.1115

$-2.115$

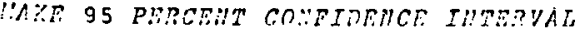

PPEOTCTIOH, YTS OP nO
?:men =
naveren $:$
wow? trat?

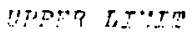

40

34.2449
$3 Y$ ANOTHR, YOE $0: \because 00$

?:O
22.450 .0

46.0333 
TRANSITION ANALYSIS: 8-7 YEAR TREND

DISTRICT 非3, KENDAL-UPPER ROCK SPRING (STRATUM 非III)

BIRTH-GRADE 1; GRADES 1-2

$21: 37$ FER $24 \quad$ DR:C2

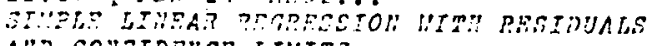
A.t.

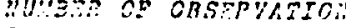

$? 8$

VISUES Y.X FOR ORSARYATOUS

1 (1)

$? 35.93$

$? 122,32$

$? 255,35$

4.2020

$?: 77,90$

$? 207.73$

$\therefore 32,59$

$?: 04,55$

?104,65

chopication

$y \operatorname{InTRCAPTA}$ ar:

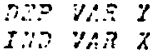

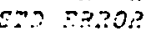

II:A:

i23.875

77.2750

23.0103

.153163

\subsection{0}

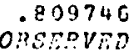

$I$

1

2

4
5
6

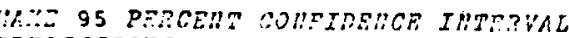

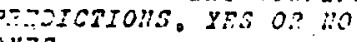

$\operatorname{sen}$

95
122
155
177

177

102

132
104

104

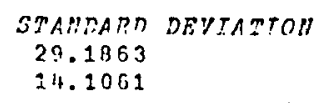

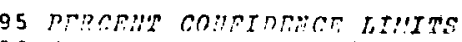
toneren

-83.4201
-1.032316

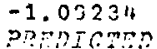
135.122 135.313

129.044

114.259 UPPR?

219.052

2.71193

$3 . C T D U A L$
-41.1224

$-13.3127$

25.3550

43.3068

$-17.9275$

15.3115

$-1.3520$

$-10.2593$

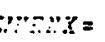

275

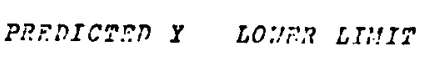

121.547

95.8600

UTPE? LI:'TS

147.234
$>$ ?: ii:

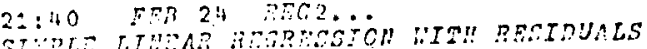

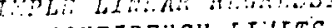

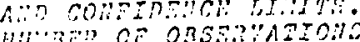

$? 7$

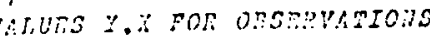

$? 80.95$

20ิ

34,122

$? 134.155$

$?: 43,177$

$? 109,102$

$? 123,132$

$? 106,104$

NEP VAR

It:? VA? $x$

ars: Baror

CORBETATIO!

ays?

108.423
125.714

15.0078

.735041

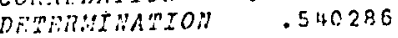

STARARD DEVTATTOE:

20.2061

y TITRRCER A 46.3327

SLOPEB B OBSTEOVED

COIII?E?
1631.05

17.7 .227

642.904

1075.48

321.335

234.442

1.22807

105.253

5043.59

MAKD 95 PRRCEH'M COHFIDEUCE IUTERVAL

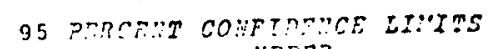

Lolis

$-21.1213 \quad 113.787$

$-2.069392-02 \quad 1.03379$

1......

ก2. $\operatorname{ses} 71$

$-12.8071$

$-12.2184$

-1. 2300

12.6320

190812

450.3174

8.70249

97.2975

sayesra

$156.0 ? 7$

245.954
334.520

98.5367

160.2188
113.549

75.7333

1226.17

PnEDTCTIOHS. YRS OR :10

?

PREDICTRD $y$

UPSER LI:II?

WI:: $\mathrm{x}=$

$? 122$

106.118
55 क 210

91.3302

120.907 
TRANSITION ANALYSIS: 8-7 YEAR TREND

DISTRICT 非, KENDAL-UPPER ROCK SPRING (STRATUM 非III)

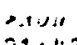

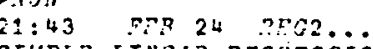

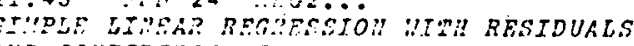
A.

$? 7$

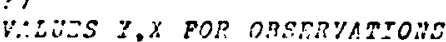

$? \frac{1}{20.35}$

5

$\because 25,80$

$?: 11: .94$

4

93.104

$? 150,143$

$? 2.142,109$

$?: 22 .: 23$

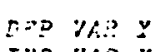

I:PA:

112.857

106.857

15.7179

Ses Fuser

cons-int

81.1620

STAMARE REYIATION

26.5420

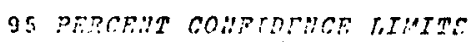

Y IVก:?CEPT A

\subsection{0}

25

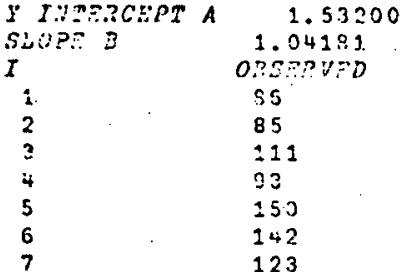

$-20.8739$

.199391

100.504

84.8770

100.081

150.581

115.000

125.090

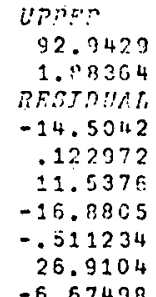

MHE 95 PERCEUT COMTINTHCA IVTSRUAL

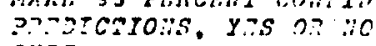

$$
\text { ?บ:3 }
$$

ans.: $3=$

PRANICTED $y$

iCHR LTIII

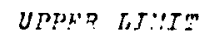

3106

ZR: K:ORIIR, y 112.954

95.7020

128.220
GRADES $2-3 ; 3-4$

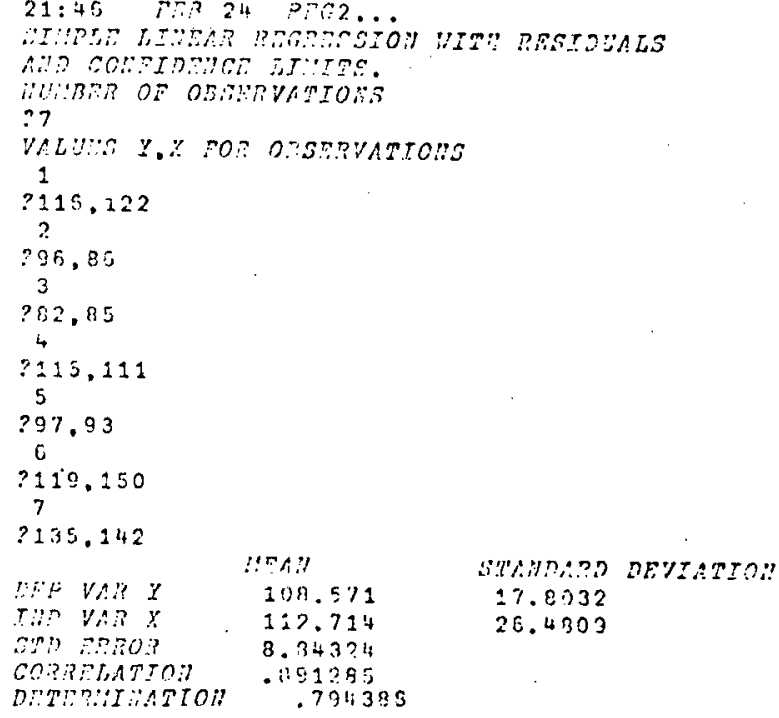

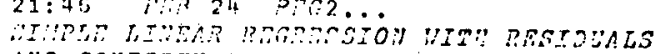

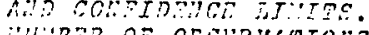

27

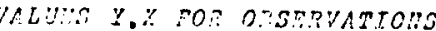

$? 115.122$

$? 2 \frac{3}{3}, 85$

? 2,85

$? 115,111$

$? 97.93$

0

$? 119.150$

$? 135.142$

Tin
VAR

!!กA."

$\lim _{\operatorname{lin} x} x$

$108.5 \% 1$

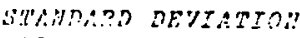

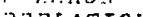

8.3437 .4

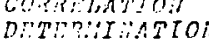

. i 91285

26.4303

IilTRICEPA A

SLOPE $B$

$I_{1}$

SCIIAREA

210,372

133.116

2014.952

2.24 .352
.261 .360

721.170

44.5553

1397.44

41.0310
.509212
On?? 1100
116
36
82
115
97
119
135

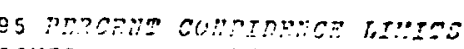
EOl:?

$243630 \quad 81.0633$

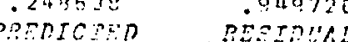

$114.130 \quad 1.86440$

32.5639

3.43610

$-9.35459$

$107.544 \quad 7.45579$

$96.7534 \quad .241613$

$130.913 \quad-11.9135$

$3,4=200$

11.8953

25.505

$5.93771=-02$

141.231

73.8592

331.215

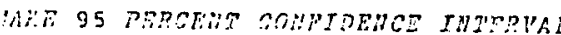

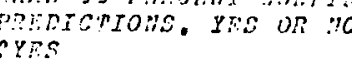

$\operatorname{mon}: 1 x=$

PAFITCRT

LOHE:S LIIIT

UPPS? II::T

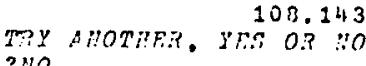

29.5464

125.740 
TRANSITION ALALYSIS: 8-7 YEAR TREND

DISTRICT 非3, KENDAL-UPPER ROCK SPRING (STRATUM 非III)

$>p: \cdots$

$21: 49$ Prn 24 RrGi.

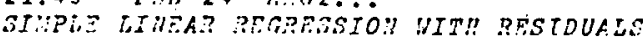

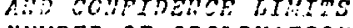

$? 7$

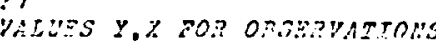

$? 104,95$

$2158,: 16$

3
$?: 21.95$

$? 117.82$

? 132,115

6

$? 109.97$

2132,119

\begin{tabular}{|c|c|}
\hline & $\therefore F A B$ \\
\hline$D E P \quad Z A R Y$ & 124.714 \\
\hline IHD VAR $x$ & 102.357 \\
\hline$s=7$ : & $13.770 \mathrm{~d}$ \\
\hline 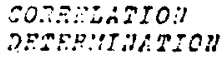 & $\begin{array}{r}.719196 \\
.517113\end{array}$ \\
\hline $\begin{array}{l}Y \\
Y\end{array}$ & 28.4373 \\
\hline$S 5 O P E \quad B$ & .936021 \\
\hline$I$ & OFS:M:PD \\
\hline 1 & 304 \\
\hline 2 & 138 \\
\hline 3 & 22: \\
\hline 4 & 117 \\
\hline 5 & 132 \\
\hline 6 & 123 \\
\hline 7 & 132 \\
\hline
\end{tabular}

STANIARE DEVTATION

18.0897

13.8976

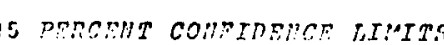

$$
\text { ines }
$$

$-79.3670$

$-.103977$

P.intertint

117.350

137.010

118.296

105.192

$\$ 35.080$

119.232

139.824

UTh?

136.243

1.97602

DESTRIAL

-13.3598
20.9337

2.713414

11.8034

$-4.0 .025$

$-10.2319$

$-7.8 \geqslant 433$

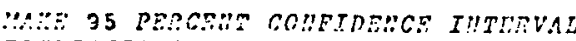

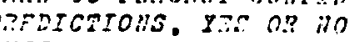

pirs

$\because x: x=$

PEADECTED Y LOMUR BREIT

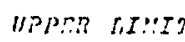
9108

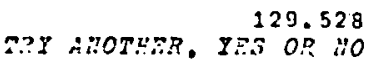

115.117

143.939 3.10
GRADES $4-5 ; 5-6$

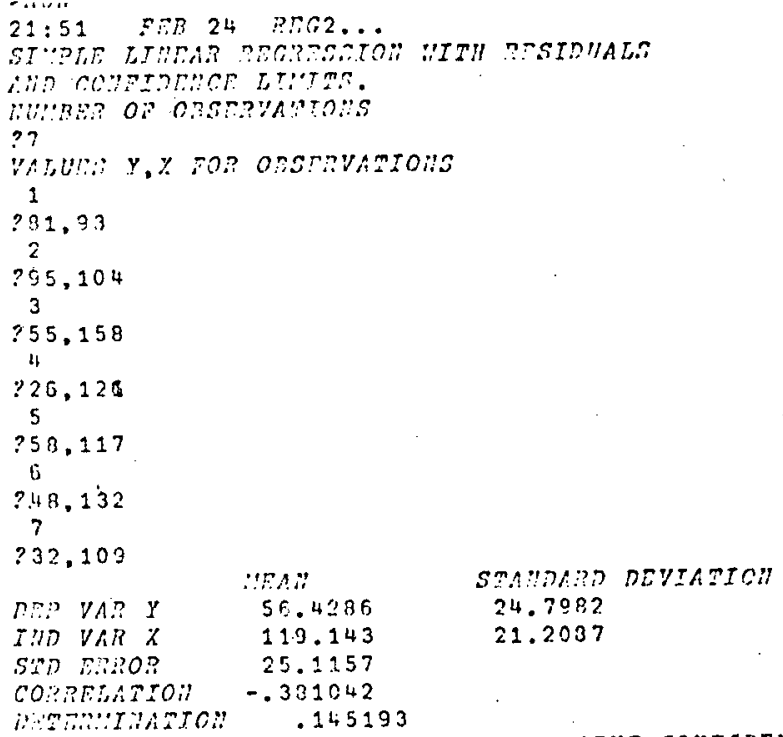
.145193

95 PEREEHT COUFINPNCT ETIITS Lo:ving?

Y THRPCOPA A

\begin{tabular}{|c|c|c|}
\hline & $\begin{array}{l}\text { SLOPE } B \\
I\end{array}$ & $\begin{array}{l}-.445533 \\
\text { onSE?VQD }\end{array}$ \\
\hline & 1 & 81 \\
\hline ก:A:STR & ? & 35 \\
\hline 179.485 & 3 & 55 \\
\hline 440.337 & $"$ & 26 \\
\hline $7.31: 33$ & 5 & 58 \\
\hline 99.439 & 5 & 48 \\
\hline 6.6435 & 7 & 32 \\
\hline
\end{tabular}

$-40.5790$

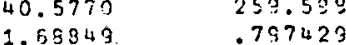

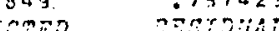

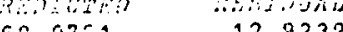
62.0751 12.9233 63.1752

33.1165

55.5012

57.3833

50.7503

60.3475

15.0035

15.0535
-20.5012

-29.5012
-516716

$-2.70030$

$-28.9475$

104.691

61.2202

348.114 PREICTIOHS, YES OR ".O

?'ss

Pnsteres

LO:IE: LI:IT

UPPR? LI:IT

$\ln \pi n x=$
$?: 30$

MRY AHOTHET, YTS OR 20

23.7928

79.4799 sctig 167.253 $2 \times 2.287$ 075.220 0 3.0 .30 $7: 29.60$

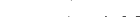

$3: 53.99$ 
TRANSITION ANALYSIS: 8-7 YEAR TREND

DISTRICT 非, KENDAL-UPPER ROCK SPRING (STRATUM 非II)

\section{GRADES $6-7 ; 7-8$}

$>\rightarrow \because \because:$

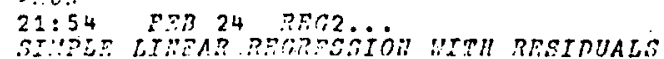

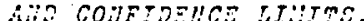

......

:?

? 2.71

5

$? 5 ? .8$

$\because 13.95$

$? 41,55$

5

$? 37,26$

6
$: 55.58$

7.58

.42 .48

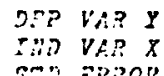

ca" zos?ort

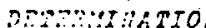

$$
\begin{aligned}
& \text { IEAM } \\
& 63.8571 \\
& 52 \\
& 15.3168 \\
& .864584
\end{aligned}
$$

.747500

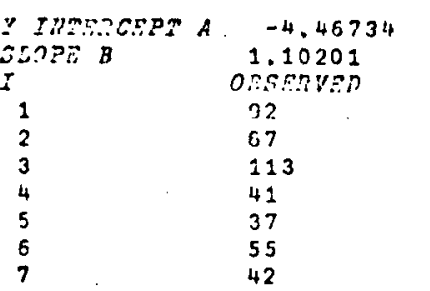

\section{STAHDARD DEVIATION \\ 28.9161
22.6863}

95 PEROEUT CONFIORHCR LTHYTS

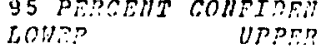

$\begin{array}{ll}-52.0735 & 43.7388\end{array}$

-52.0735
-395598

piिएक

73.7752
84.7953

100.223

56.1431

24.1849

53.4491

48.4250

43.7358
1.83842

TECIRIIAL

18.2248

$-17.7953$

$-15.9431$

12.8151

$-4.44911$

$-4.421,911$
-6.42903

MAR 95 PRRCEIT COHETDEHCE IUEAVHL P.PDTCTIOHS, YES OR 110

?YES

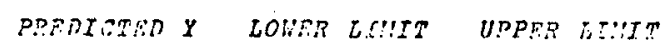

$7: \because 1 \%=$

52.8371

35.7063

69.3678

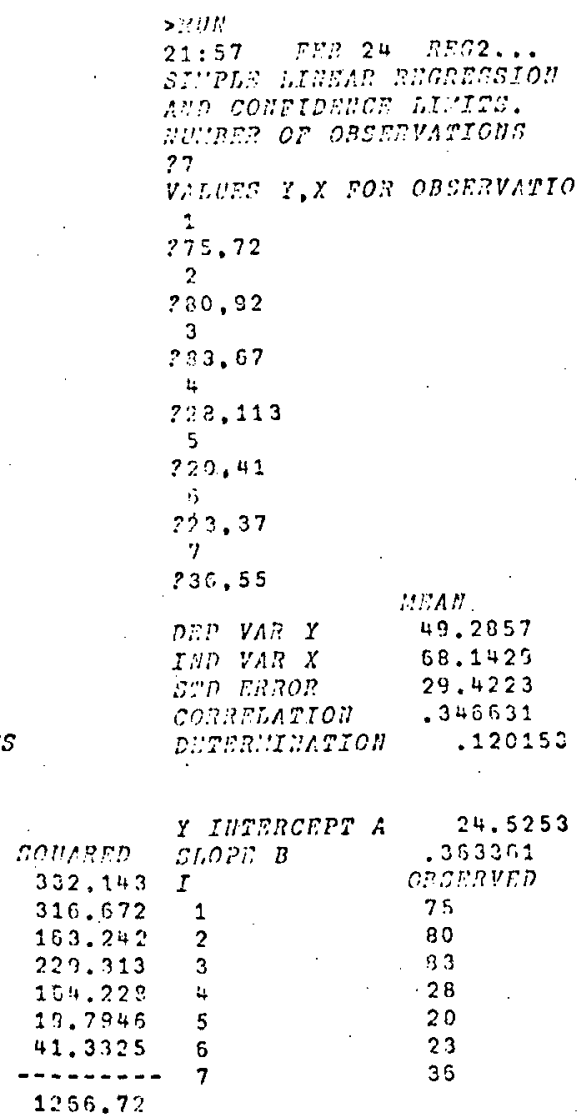

UARE 95 PERCEUI COHETDEHCE IUTERVAL

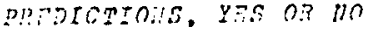

oy-5

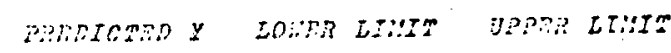

$\min 3 x=$

$\begin{array}{llll}253 & 43.7834 & 10.4537 & 77.1030\end{array}$

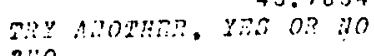


TRANSITION ANALYSIS : 8-7 YEAR TREND

DISTRICT 非3, KENDAL-UPPER ROCK SPRING (STRATUM \#III)

GRADES 8-9

$21: D y$ AH 24 nEG2

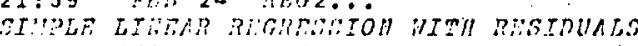

AII COUEFDAMCE EI:IISS.

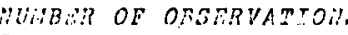

$? 6$

VALIES Y, $Y$ FOR OBGRRVATTOULS

?. 33,75

$? 38,80$

3.

$? 23.83$

$? 12,28$

5

$? 21,20$

$? 11,23$

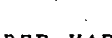

IEAR:

31.3333

$\begin{array}{ll}\text { IUP VARX X } & 51.5000 \\ \text { SID R?ROIT } & 24.2399\end{array}$

STAMTAPD DEVTATEOH

27.1135

CORRELATIOL! E.0U725

CETERMTHAIOH $\quad .350070$

95 PARCRNT COHFIOENCE LIUITS

LOMB: U?

$y$ IUTRRAPA A

4.00725

$-53.4001$

51.4740

3.443515 $\quad \frac{1}{3}-51072$

SQUSTRD

3.8325
40.4555

39.1375
-8.45554

1230. 83
38

48.0473

$-8.45554$

1535.144

$\begin{array}{lrrr}23 & 13.5641 & -6.85415 & 47.2165 \\ 21 & 14.5193 & 6.39068 & 40.7139\end{array}$

$5 \quad 2$

$5.211: 3$

-........

WAKE 95 PERCEUT COIFIDEHCF THTERVA

P.RTICTIOLI, YTS O.? 110

Y YES

PREDICTRD $y$

LOUER WIVT

UPPEP LTUTT.

Halenx

274.3538

MRY AMOTHER, YES OR 110

$-1.08372$

55.7913 
TRANSITION ANALYSIS: 8-7 YEAR TREND

DISTRICT \#6, JERICHO (STRATUM \#IV)

BIRTH-GRADE 1; GRADES 1-2

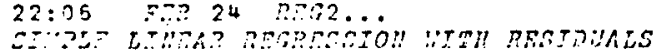
$\therefore-0$ -

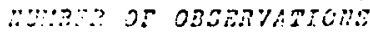

"REUES Y.X FOR ORGERVATIOHS

?100,1:0

2

$? 2.3,105$

4
$?, 150,107$

250.91

8.9

: 12.72

$?: \div 1,73$

221,80

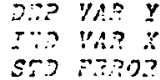

constriston

Dessovintion

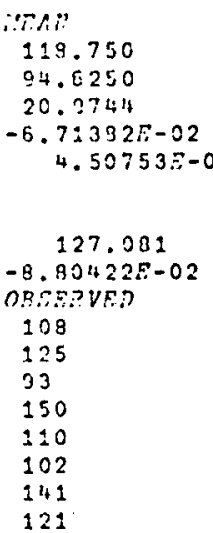

STAHAARD DEVTATIOH

29.4524
14.8414

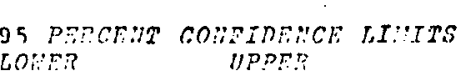

\begin{tabular}{|c|c|}
\hline y $I: A P S C E P A$ A & 127.081 \\
\hline $\begin{array}{l}\text { SLCBZ } P \\
I\end{array}$ & $\begin{array}{l}-8 \cdot 90422 E-02 \\
0 R E S ? \forall F)\end{array}$ \\
\hline 1 & 108 \\
\hline 2 & 125 \\
\hline 3 & 33 \\
\hline & 150 \\
\hline 5 & 110 \\
\hline 5 & 102 \\
\hline 7 & $1+1$ \\
\hline & 121 \\
\hline
\end{tabular}

2.07552

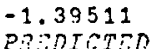

117.396

117.572

117.837

17.660

119.083

120.302

120.6511

119.503

252.08

1.21903
DFit

PFSTRIAL

$-9.39635$

7.42755
-24.8356

$-24.8365$

$-3.06015$

20.31651

1.19004

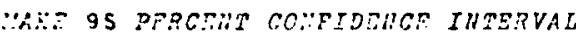

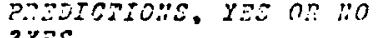

?.35

PRERTCTED $Y$

LOEER EIITT

:a:m

293

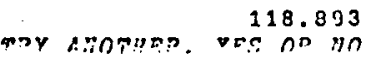

100.623

137.163

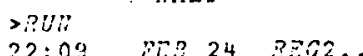

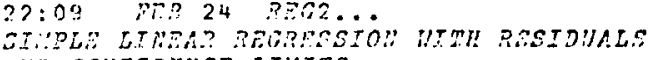

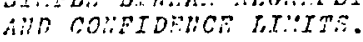

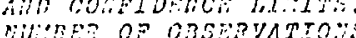

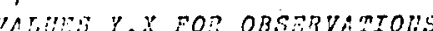

$? 94.108$

2

? 83.125

3

396,93

$? 113,150$

5

$? 1: 7,110$

$? 81,102$

$? 110,141$

DRP VAR Y

T:i) VAR $X$

cospetsinton

$\because E A H$

100.286
118.1129

STABDARD DEYTATTON

118.129
14.5702

15.7238

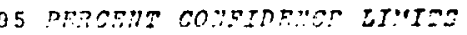

LC:IF:

-34.1971

$y$ IUMERCEPT A

.274603

sringern

38.2914

55.17287

615.855

10145.94

82.2495

413.953

2.22200

2639.55

$I$

1

53.8159

-34.1971
-.340883

ritioremen

96.9237

102,054

122.074

96.9785

93.3324

141.819

1.12556

$-2.19368$

$-10.95,43$

5.05912

$20.02: 5$

$-12.9394$

8.85756

4.81224

394.589

32.4002

$.0532 \cong$

400.262

164.843

78.4564

1076.08

TAKE 95 FERCEUT COUFTDEHCR IUTEZWAL

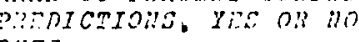

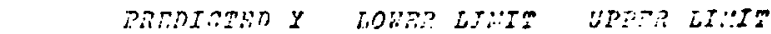

$\operatorname{tin} x=$

$y=100.510$

36.2481

114.772

TS OR $\because 0$ 
TRANSITION ANALYSIS: 8-7 YEAR TREND

DISTRICT 非6, JERICHO (STRATUM 非IV)

GRADES $2-3 ; 3-4$

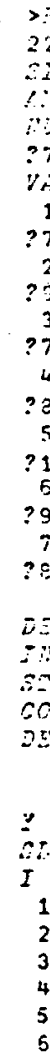

$22: 12 \quad 50 \quad 24$, $202 \pi$

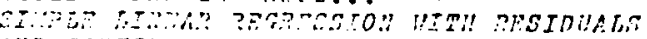

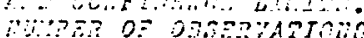

.7

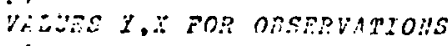

$?+\frac{1}{7+.93}$

$2 \div 2,34$

3

$? 71.83$

2.27 .96

5

$? 1: 0,113$

$? 95.117$

?ะ. 81

- -2 Vis $y \quad \therefore E A$

88.1429

an?

conezitrian .715954

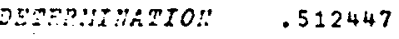

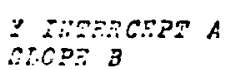

Arcos 3

2

4
5

7

13.1330

13.7443

$-52.8437$ $-8.31155 \%-02$ PDEDICTER 85.6022

86.2052

78.7620

$87.55: 3$

39.2820

102.019
77.3940
SEAINATD REVIATEOH

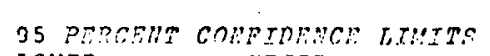

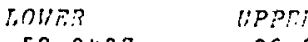

26.3207
1.45115

1. 45115

21.6025
-11.023

5.72376

$-7.76204$

$-.654273$

10.7174

$-7.018055$

10.6050

$\because$ YY 95 PRPCSUM COMFIDENCE IIITERVAL pnerictions. zas o.

$\operatorname{bin}$

W::nz:

PRIDECEES Y

I, ח:E? LI:IT

UPPF? LETIT

$2: 01$

TRY AHORZER, YRS OS 91.074

80.7740

101.375

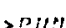

$22: 14$ EER $24 \quad$ REC2.

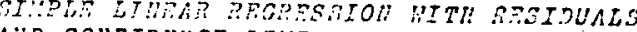

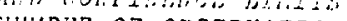

Whinter of 0

ALUES Y,X FOR OBSFRVATIOHS

1

$? 35,90$

$? 83,74$

$? \stackrel{3}{104,02}$

47.9

79.71

.80 .87

? 80

$? 91.110$

$? 84.95$

DOP VAR $y$

I:I) VAR X

STI Th:ROR

Ma:"

86.7143

88.4285

8.20552

THTER:THATSOH $\quad .240777$

บ IUTRPCRPT A

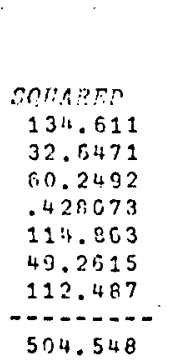

SEOP: B

1

30

58.3499

320760
OSTRVE

83

83
104
79

79

$\begin{array}{ll}4 & 80\end{array}$

5
7

93.6335

TTYDARA DEVIATIO:

B. 53673

13.1511

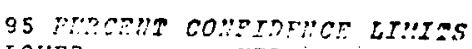

Loliti

-.102273
-.334140

$\sin 334140$

87.2183

82.0852

7.8599

3.822

U⿻?

115.308

RPF? 9560

$-1.21834$

.913222

16.1401

$-2.12390$

$-6.25805$

$-2.63354$

$-4.82214$

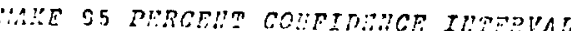

PREIICTIOHS, YES OR VO

byos

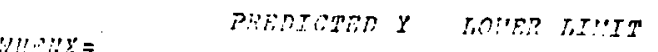

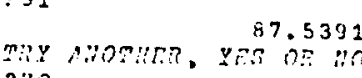

79.3894

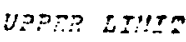

$?: 0$ 
TRANSITION ANALYSIS: 8-7 YEAR TREND

DISTRICT 非6, JERICHO (STRATUM \#IV) GRADES $4-5 ; 5-6$

$>n: 3$

$22:: 7 \quad \therefore \because 3 \quad 24 \quad>0 \% 2$

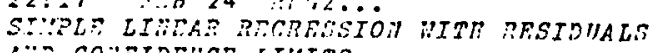

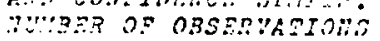

VELUES Y,Z FOR OBSERVATTOWS

1

$? 22.95$

233,86

3

59,83
$.79,204$

$? 79,104$

$? 202,79$

792,79

$? 95,80$

? $19.9:$

201

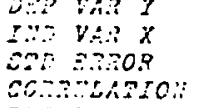

74.4280

34.4280

23.5352

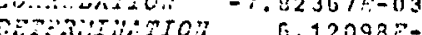

\begin{tabular}{|c|c|}
\hline$y=$ 요 & 75.0652 \\
\hline $\begin{array}{l}S \leq 0 D E Z Z \\
I\end{array}$ & $-1.85000,0=02$ \\
\hline : & $2 \hat{\varepsilon}$ \\
\hline 2 & 33 \\
\hline 3 & 59 \\
\hline 4 & 70 \\
\hline 5 & 92 \\
\hline 6 & 95 \\
\hline & 81 \\
\hline
\end{tabular}

STAIDART DEVIATTOH

21.6322

9.14435

35 PROCERT GOHETREICE LIUITS

yptr?

$-165.551 \quad 317.681$

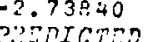

74.2334

74.4735

74.5290

74.1404

74.5031

74.3190

2.70130

7.71150

$-4: .4735$

$-15.5090$

4.85752

120.3915

6.61302

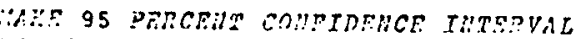

P2ERTCTIONS. THS OR HO

PYZS

?::1\%

PRENTCIED

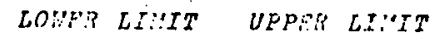

74.4365 $?$ ?no
$>R U: !$
$22: 13 \quad$ FFB $24 \quad$ REC2.

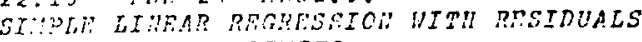

A.t) COUPIDFMCE LT:ITS.

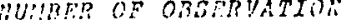

YLUES $Y, X$ FOR OBSTRVATTOIS

130

230.57

$? 26,82$

3

$? 30,33$

$? 39.59$

5

$? 18.79$

$? 0,0.32$

$?+73.95$
WFAN STALMARD DFVIATTOH
DAR VAR Y $y 3.8571$
72.428
27.7274
21.5705
COARELATIOH .482485

DETER!IIHATOH $\quad \mathbf{2 3 2 7 9 2}$

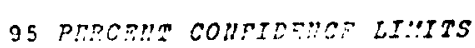

LOIS? UPF

$y$ TITERCEPT A 8.93075

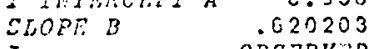

$-83.3255$

$-.674360$

PREDTCTED

50.4903

59.7934

29.4034

45.5287

57.9328

65.9954

67.9500

155.939

1.91477
85.971100

RACIRUAL
39.5097

4.20653

.596561

$-15.5287$

-39.0328
$4.605475-09$

21.1 .153

302.653

415.739
43.8114

0

MAKT 95 PRPCHUM COHFINEWCE TUTERVAL

11.1440

seysets

1561.01

17.6958

-355?95

$150 \% 53$

$2.212103=-05$

PREDICTIOHS. YES OR 110

?YF.S

PREDTCMER y

CONER LI:TY

IPP:P LI:YTT

!nt?

$? 74$

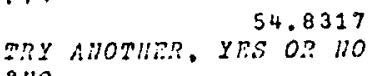

25.8989

80.7646 
TRANSITION ANALYSIS: 8-7 YEAR TREND

DISTRICT \#6, JERICHO (STRATUM \#IV)

GRADES 6-7; 7-8

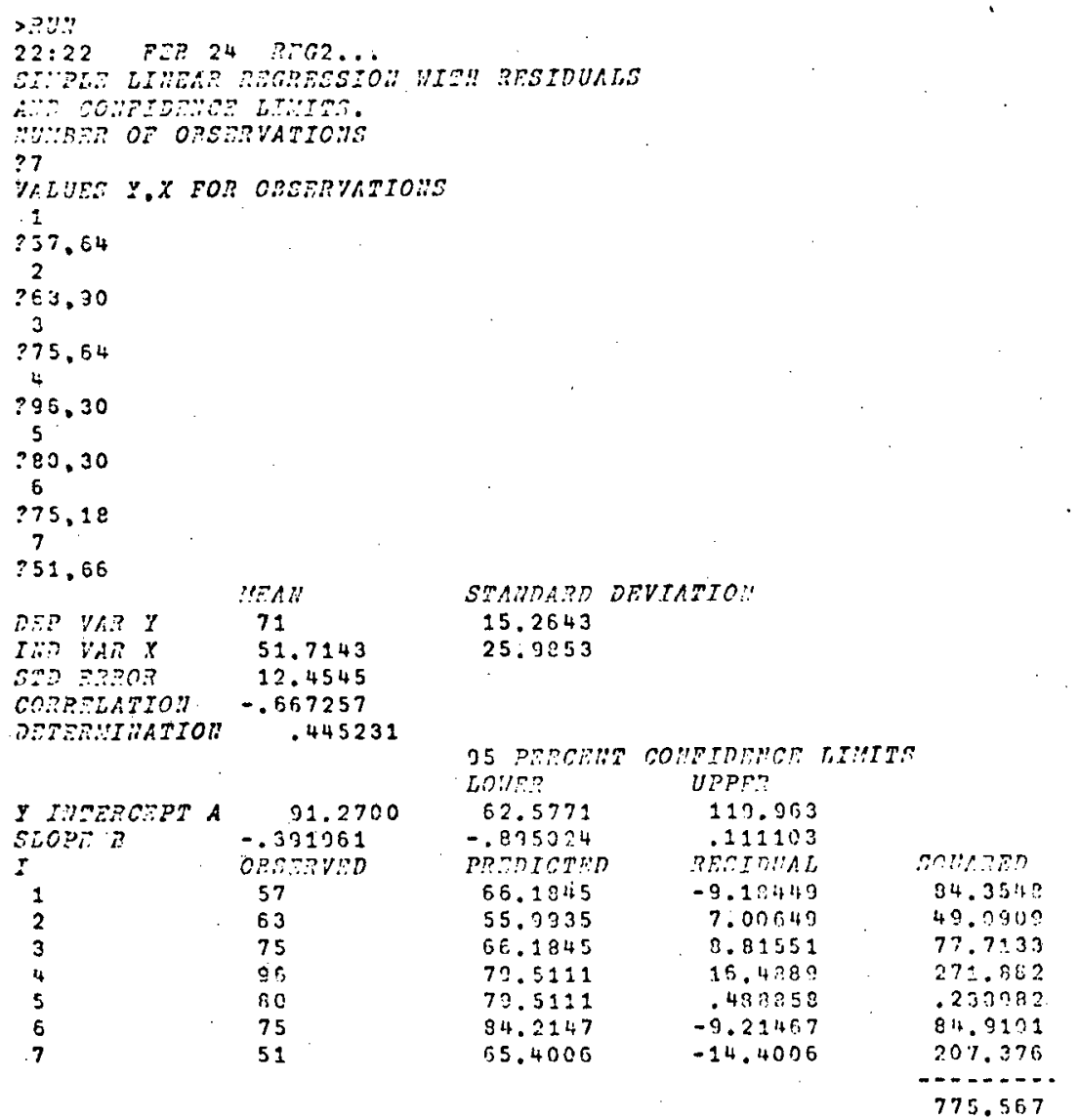

WAE 95 PERGEAT CONEIDEUCE TLTERVAL PaEDICETOUS, YES OR

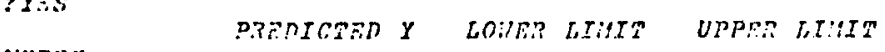
$\operatorname{tin} x=$

$? 55$

$69.7121 \quad 57.4972 \quad 81.9271$

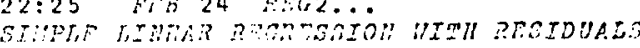

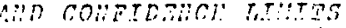

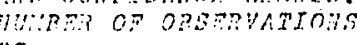

$? 7$

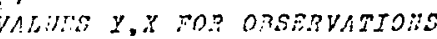

1
$? 43,47$

$2.050,57$

$? 60,57$

247.63

$? 57.75$

5

$? 52.96$

?. 42.80

7
$? 39,75$

DIP VAP

:EA:

IIID VA:

49.4286

70.4285

8.18022

CMAMTADD DEYYATOO

7.59072

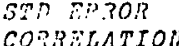

$-.179460$

DFFEIIIIATIOH 3.22059 P-02

Y IDTERCHR' A
SLOPE $B$

1

2
3
4

4

5

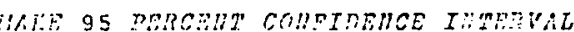

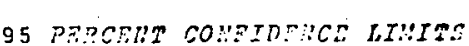
LOHE?

17.1581 $-.514550$

51.4005

50.5589

50.0538

49.0439

48.2530

49.0439

iPPER

33.5545

0.446327

PESTRIS:

-2.40047
-3.44119

$-3.05381$

7.95519

$-5.62208$

$-1.02298$

SQLET $5.7523 ?$ 89.1251 63.3010 2n 3132 43.263 100.378 100.378

334.580

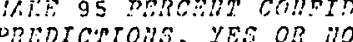

?YPS

men:?X $=$

PRTOICTED $y$

LOMSP LIIIT

UPPER LI:YYT .70

T?Y MORIER, YIS OS 46

41.5123

57.4170

$? 110$

TPY A:OOMER, YES O? 
TRANSITION ANALYSIS: 8-7 YEAR TREND

DISTRICT 非, JERICHO (STRATUM 非IV)

GRADES 8-9

780 HAET

$22: 27$

SIVIFE LIMTAR RECRESGIOH WITH RESTDUALS

ATD CONEIDHICE LTHITS.

$? 6$

VALURS $Y, X$ FOR OBSERVATIOUS

1

$? 54.49$

$? 49.60$

3

$? 42.47$

733,57

5
-27.52

$? 32,42$

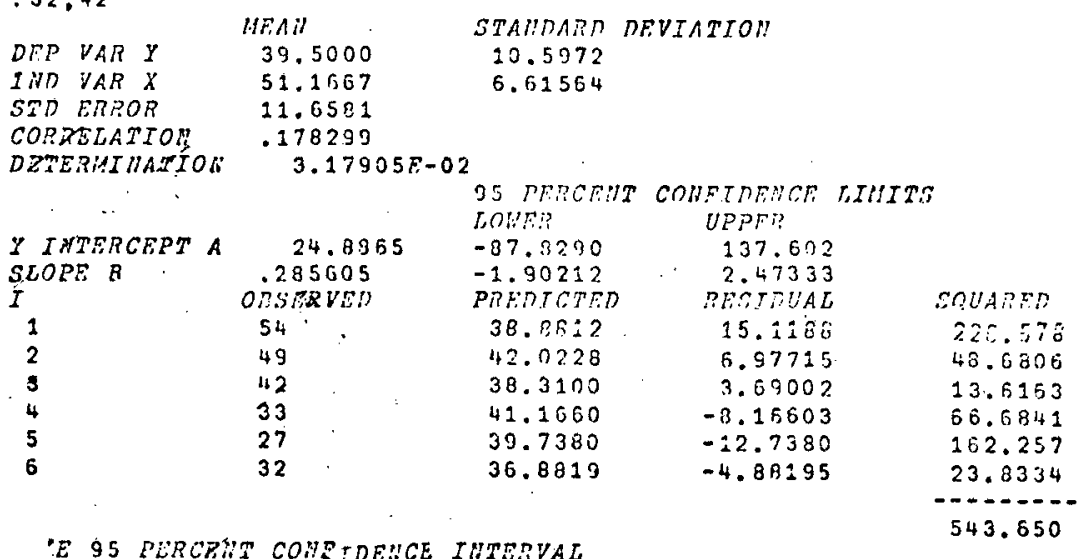

E 95 PERCRYT COHETDENCE THTERVAL CTIOHS, YES AN HO

$Y E S$

\begin{tabular}{|c|c|c|c|}
\hline $\begin{array}{l}\text { WaRnX = } \\
? 49\end{array}$ & PREDICTED Y & LCWER LIMIT & UPPER LIHIT \\
\hline RY ANOTHER. & $\begin{array}{r}38.88 T_{2} \\
\text { YES OR } 10\end{array}$ & 24.8445 & 52.9173 \\
\hline
\end{tabular}


TRANSITION ANALYSIS: 8-7 YEAR TREND

DISTRICT 非, LUCEA (MIXED STRATUM)

BIRTH-GRADE 1; GRADES 1-2

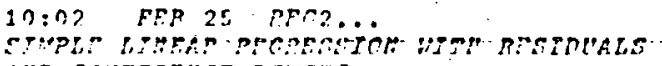

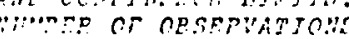

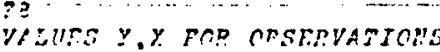

1

9254,535
$2,29,529$

3.

2231.503

$? 243.481$

$? 249,517$

2205.510

$? 218.614$

$\because 139.601$

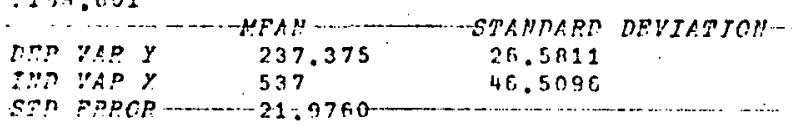

$21.9740-10$

COPBELAMTOH $=.643523$

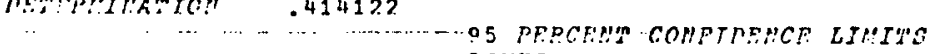

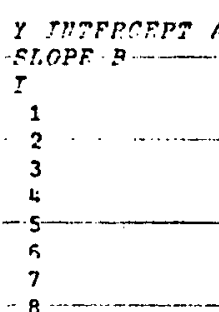

434.876 LOTPR TRPER

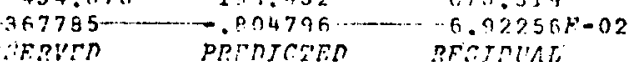
PROTCERA

254
202
231
243

231
243
249
285

285 $23 ? .211$ 340.317 247.673 257.971 15.8894 $-\$ 9.3173$ $-18.6730$ $-14.9710$ 247.30502000 $200.056 \quad 8.24444$ $213.037 \ldots-15.3368$

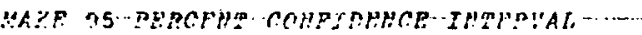
nurryctins, YEs OP llo

PES

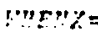

$\$ 536$

237.749

$256.76: 0$
$10: 09$ FFR 25 REC2..

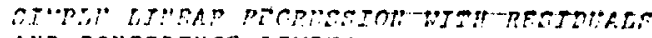

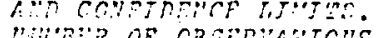

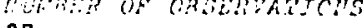

VALIRE Y,X RCR OBSERVATIOUIS

$? 200.254$

$? \because 96, ? 2 ? 1$

2713.231

2232.243

$? 204.249$

$? 279.285$

2277,218

nTE $\forall A P Y$

nes.

228.714

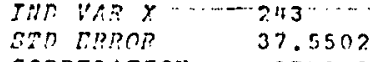

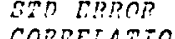

CORERTATENM . 274242

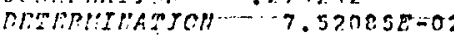

35.61553

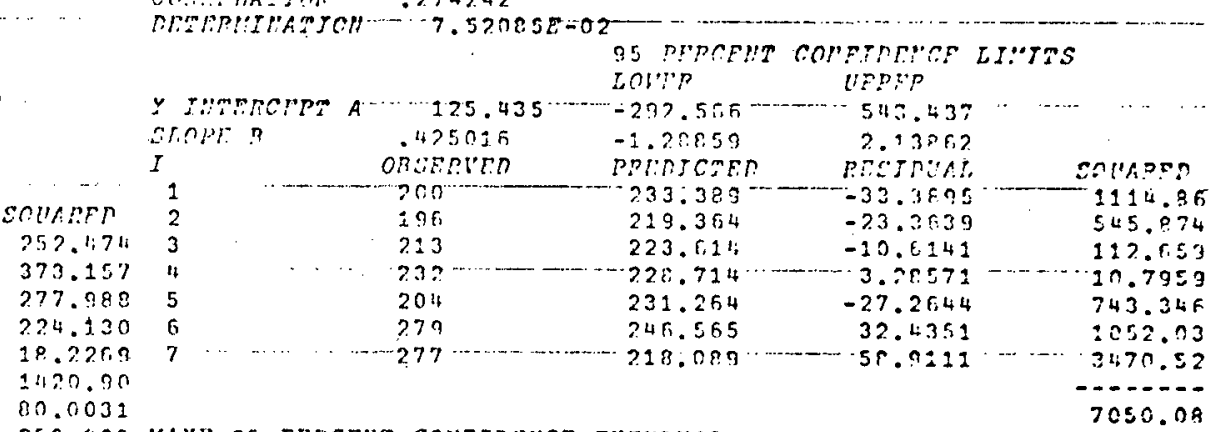

80.0031

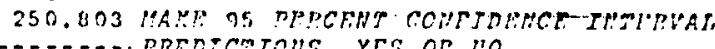

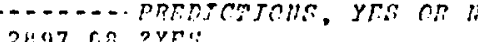

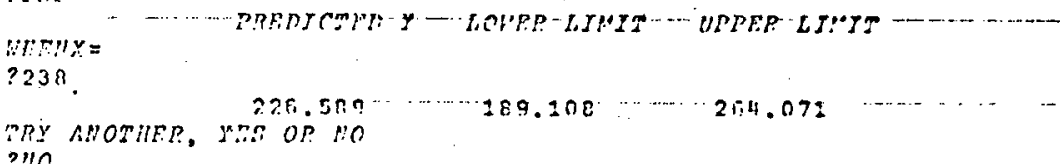


TRANSITION ANALYSIS: 8-7 YEAR TREND

DISTRICT 非 1 , LUCEA (MIXED STRATUM)

GRADES $2-3 ; 3-4$

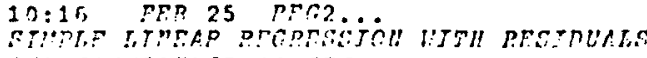

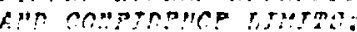

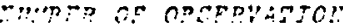

$? 278.259$

3280.200

3290.196

2242.213

$? 260.23 ?$

2215.204

$? 308,27$

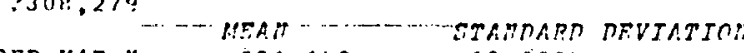

$\operatorname{tin} \operatorname{sip} x$

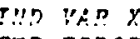
281.143 20.0894
32.0499

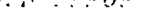
2.26 .143

DRER"'THATIOH 5.65075F-02

\begin{tabular}{|c|c|c|c|}
\hline & & Loler & IDPF? \\
\hline IMIPPCF? A & 239.060 & 39.8 .482 & 438.471 \\
\hline $\begin{array}{l}\operatorname{Sr\cap PF} B \\
I\end{array}$ & 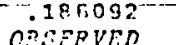 & $=$ & $\begin{array}{r}1.05039 \\
050000\end{array}$ \\
\hline 1 & 278 & $28 ? .257$ & -9.25730 \\
\hline & $200 \cdots$ & -2.76 .278 & -8.27739 \\
\hline 3 & 288 & 275.534 & 12.4505 \\
\hline 4 & 242 & 278.697 & -36.6072 \\
\hline 5 & 2.53 & 282.233 & -13.2320 \\
\hline c & 315 & 277.022 & 37.9777 \\
\hline$?$ & 308 & 290.973 & 17.0200 \\
\hline
\end{tabular}

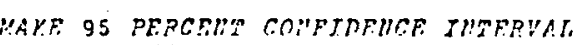

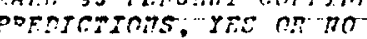

PRRIICARA Y LOWRR LIHIT

UPPER LIIIIT

2227

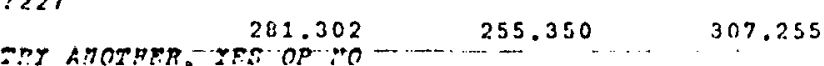
7110

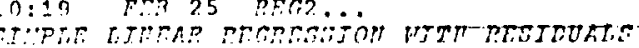

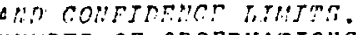

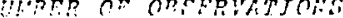

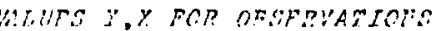

$\because 194,257$

$? 165,278$

$? \pm 214.208$

$?: 99.288$

$? 205,242$

$? 241.263$

$? 265.315$

man

GTARIRART DRVIATIOH

$\begin{array}{ll}\text { DEP VAR Y } & 203.571 \\ \text { IES IAP X } & 273.057\end{array}$

30.0824

CMP FRROR

$31.1,868$

CORPRLATIOI! .29503

DETERHTHATOH - 8.70434R-T2

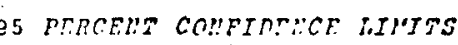

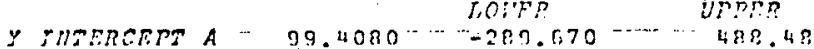

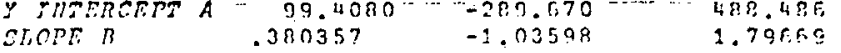

sonmpen

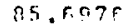

60.5234

155.1113

3346.608

175.103

141.2 .51

$2 P 9.710$

3563.44

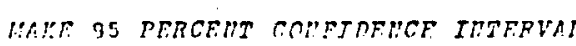

PRERTCSIOHS, YTS OR 110

:H!r:!: =

$? 2 B 1$

TRY AHOTHER, YTS OR 280 -1.03598
$0 p r n=c m 50$

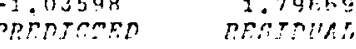

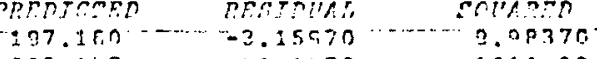
$209.1474 \quad-401472 \quad 1611.90$ $200.95 \ldots \ldots-7.34362 \ldots . .53 .9290$ $101.454 \quad-28.9508-1030$ $\begin{array}{lll}131.454 & 13.5457 & 122.455\end{array}$ 39.2760
$219.220 \ldots \ldots$
26.7796 717.147 4957.09 
TRANSITION ANALYSIS: $8-7$ YEAR TREND

DISTRICT 非1, LUCEA (MIXED STRATUM)

GRADES $4-5 ; 5-6$
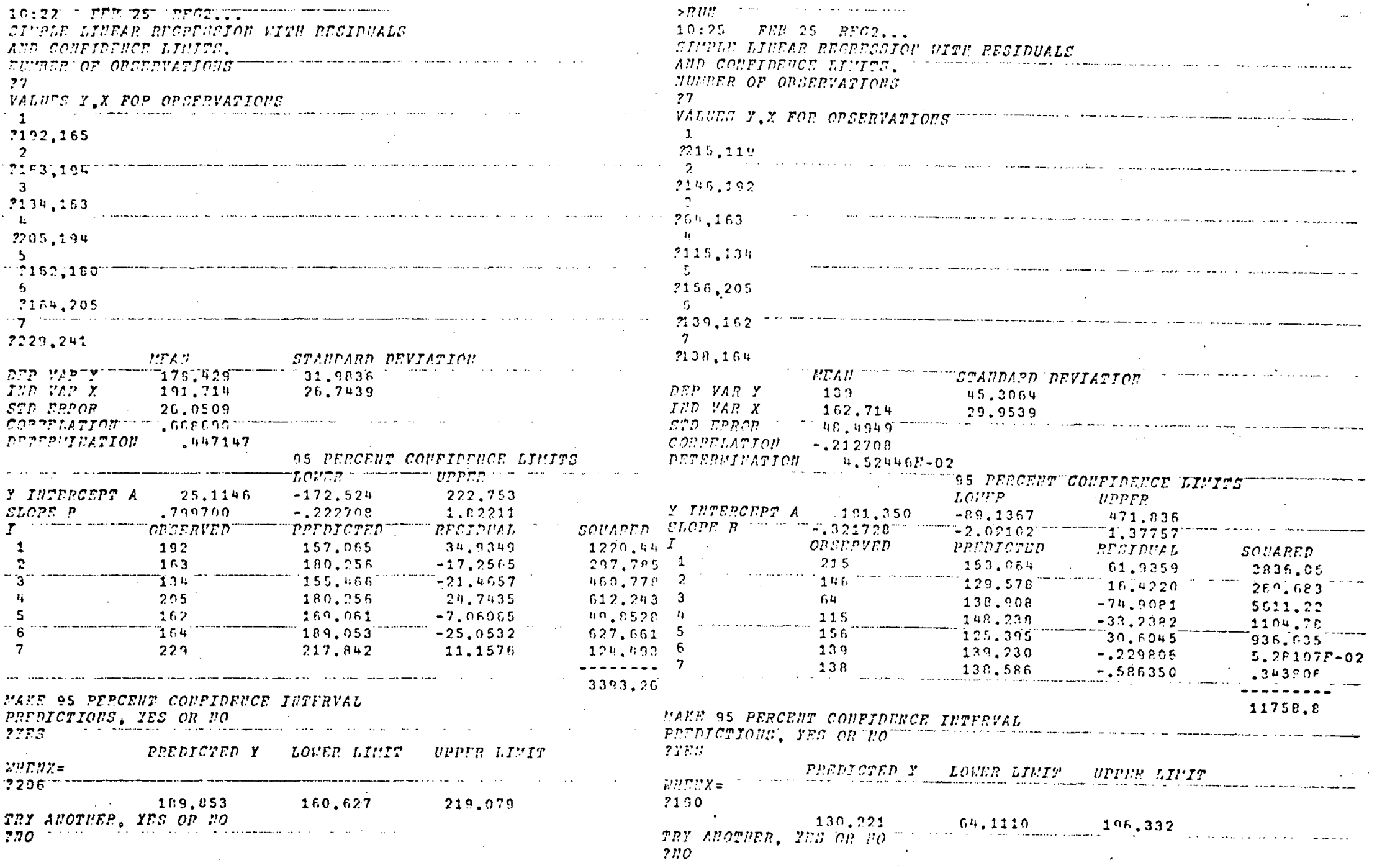
TRANSITION ANALYSIS : 8-7 YEAR TREND DISTRICT 非, LUCEA (MIXED STRATUM) GRADES 8-9

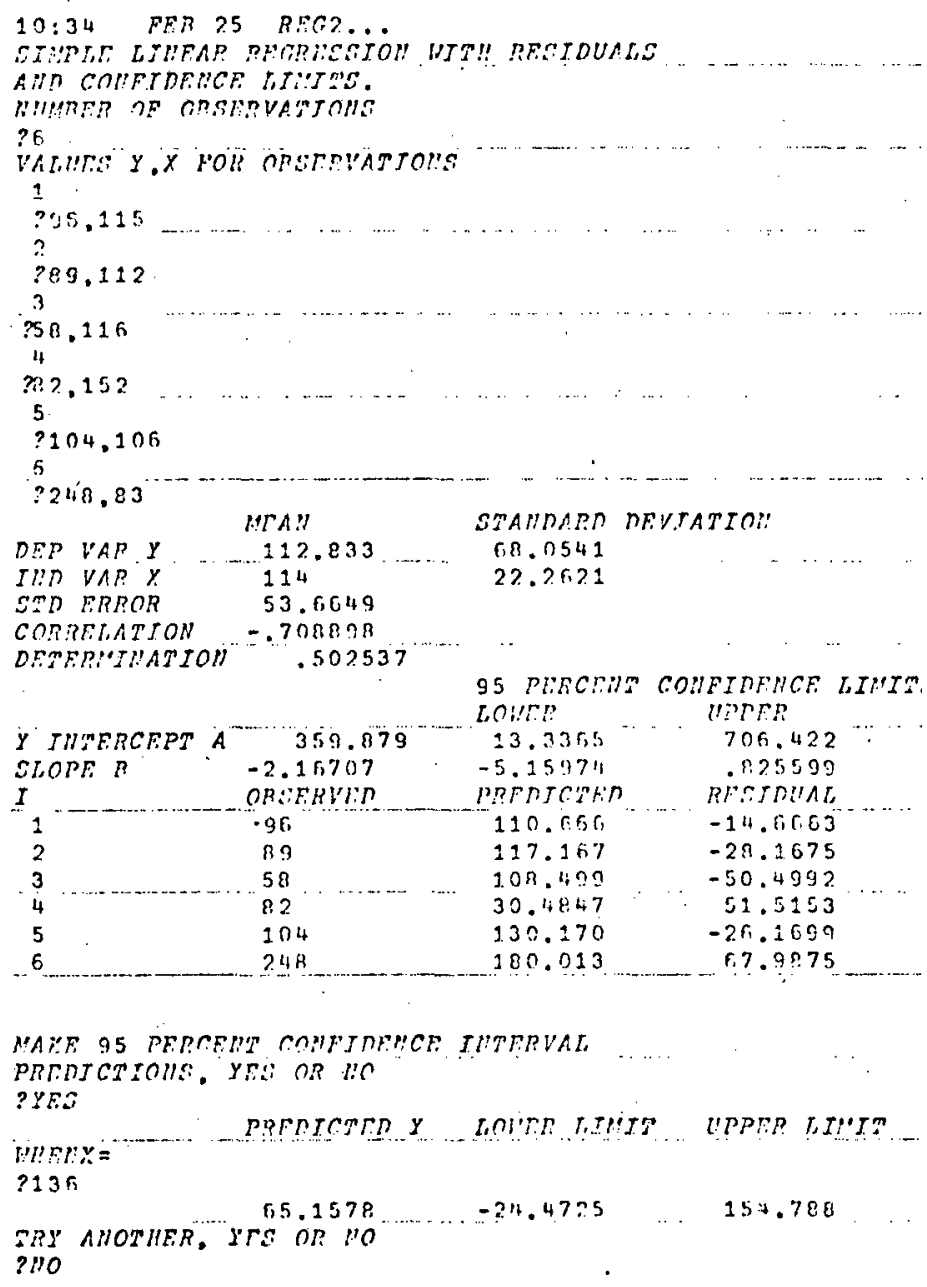

95 PINCRNA CONFIDFNCE LIMIM L, OI!I:?

$23.3,35,2$ $-5.1597 \%$ ?RPNTCER i)

706.422 .825590 RICIDIAL $-14.0603$

$-28.2675$

$-50.4992$

51.5153

$-26.1699$ 6.9 .9075

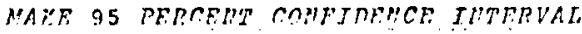
PRTDTCTROII: YRai OR HO ?XES

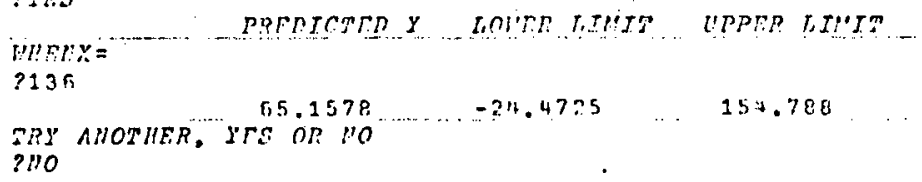




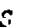
_. - - .

\title{
SOLAPTA
} $215.099^{\circ}$ 793.407 2550.17 2553.83 684.863 4622.33

11529.7 
TRANSITION ANALYSIS: 8-7 YEAR TREND

DISTRICT 非, GREEN ISLAND (MIXED STRATUM)

BIRTH-GRADE 1; GRADES 1-2

$22: 23$ FFR 24 PEG 2 .

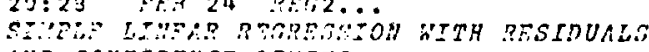

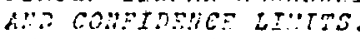

UU:?E⿱

VALITS Y.X FOR ORSERVATrONS

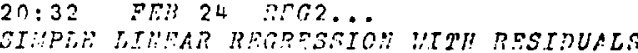

An: COHETDENCE LI:IIme.

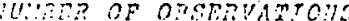

3345,443

2

?. 16,430

$? 426,4: 4$

5

6

7.0 .336

8.346

$? 439.337$

IEA: STAMRPD DFVIATIO

$\begin{array}{llll}D E P Y A R & 394.125 & 48.9852 \\ I \cdots T & 391.625 & 46.0898\end{array}$

E:? $5730: ? \quad 51.9464$

.

ocitition -.193921

Protaithaton $\quad 3.605668-02$

\begin{tabular}{|c|c|}
\hline 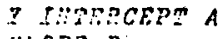 & 473.205 \\
\hline$\frac{3}{2}=0389$ & $\begin{array}{r}201327 \\
0859070\end{array}$ \\
\hline 1 & .345 \\
\hline 2 & $33 \%$ \\
\hline 3 & 416 \\
\hline 4 & 425 \\
\hline 5 & 468 \\
\hline 6 & 355 \\
\hline 7 & 355 \\
\hline 9 & 439 \\
\hline
\end{tabular}

95 PERCFUT CONPIDHUE LTITTA

62.5072 bes?

62.241433
-1.93 .902

-1.211433

383.751

304.553

356.376

$39 ? .607$

395.201

405.352

402.334

1105.155

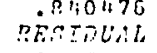

$-38.7510$

$-45.5537$

$29.623 ?$

36.3931

72.7332
-50.3572

$-37.9341$

$33.8414 ?$

OUE 95 PERCENT CONETDEMCF IUTERVAL PATRECTIOAs. YTS OR :10

?yes

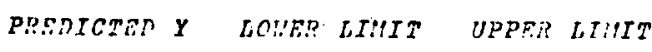

$3: 1: x=$
$? 387$

$395.059 \quad 349.860 \quad 440.258$

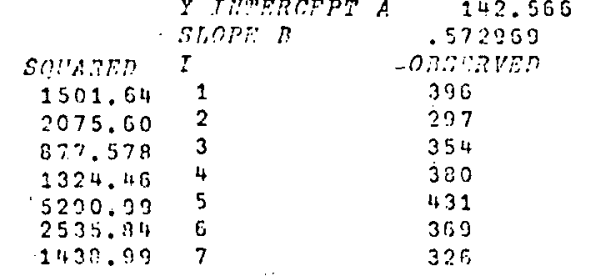

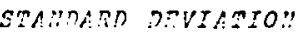

1145.47

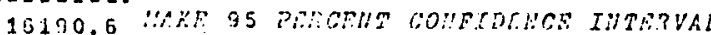

prentemsiar. yos on 10

?\%:

$\because \cdots x=$

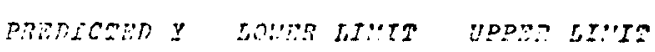

$? 325$

35.8 .889

mox nomen, yas or

331.837

405.281

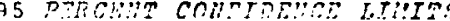

rones?

$-170.734 \quad 455.917$

$\begin{array}{lll}355.803 & -30.0025 & 1504.24\end{array}$

$\begin{array}{lll}320.321 & -2 E . ? 211 & 724.747\end{array}$

30.001 -E.55Ce1 $\quad 14.2332$

20.2345

520.300

$8: 0.4 ? 5$

7364.68 
TRANSITION ANAIYSIS: 8-7 YEAR TREND

DISTRICT $\# 2$, GREEN ISLAND (MIXED STRATUM)

\section{GRADES 2-3; 3-4}

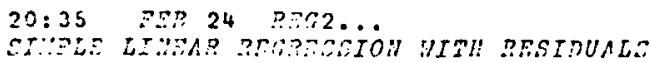

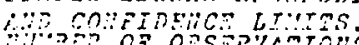

$?$

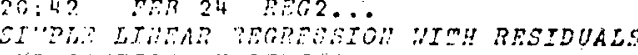

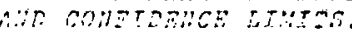

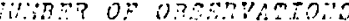

$: 7$

VILUES Y.X FOP ORSTRVATIONS

$? 291,320$

2

2337,335

3

$? 273,297$

$? 358,354$

$? 380,320$

$?: 4: 4,431$

2335.259

DEP VAR $Y$

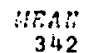

I:T $\forall: B x$

342

STAMARE DEVIATIO:

rem

363.857

47.4833

45.3116

nomentont .394086

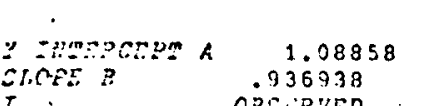

95 PFRCEIT COMPZNMUCE LIIITS

LoWre? UPQ?

2.0.7.?
$-136,57$

$\begin{array}{r}136.574 \\ .397270 \\ \hline\end{array}$

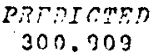

372.110

277.359

277.359

357.125

357.125
404.909

340.829

138.751

1.47650

-3.00300
-9.21502

$-35.1159$

$-354031$

-354535
25.2355

22.8752

3.09134

$-11.8135$

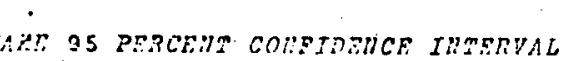

PRTDICTIOHS. yes on :10

?יㅗ

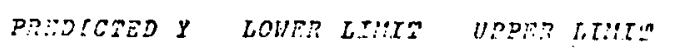

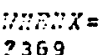

aromiso $\quad 346.819$

$2: 10$

324.010

369.627

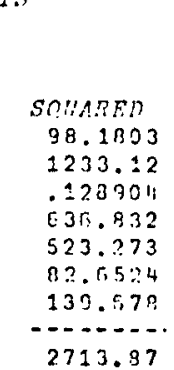

1

$? 212.242$

2

2255.291

$? 234,337$

$? 234,279$

5

$? 312.353$

$\because 335,350$

$735: 350$
7334,414

T.S $V 19$

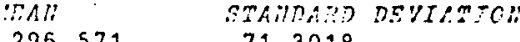
236.571
71.3018
322.712
60.8871

Int VAR $x$

sto ERt:or?

consRtamroi

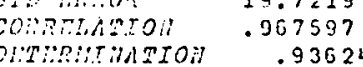

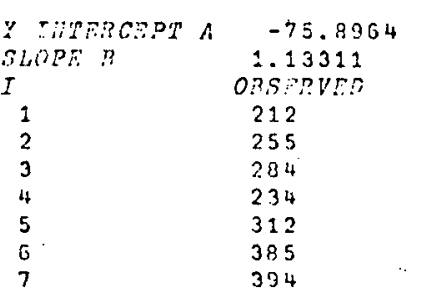

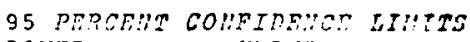

$\begin{array}{ll}\text { LOWER } & \text { UPPED } \\ -139.283 & 37.4909\end{array}$

$\begin{array}{ll}.793127 & 1.47308\end{array}$

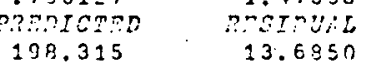

13.6950

305.260

$21.96 \%$

$-6.2399$

$-17.755$

354.634
30.3155

$393.203 \quad .732892$

sadsen

$\$ 97.278$

1.35215

422.242

38.9366

315.2 .48

019.099

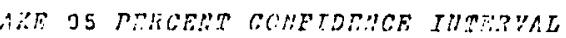

.625520

1944.77

2713.87 ponforiolls, yet on WO

$$
\operatorname{x\in S}
$$

$\because \cdots+x=$

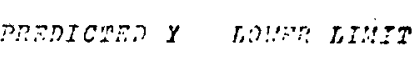

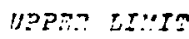

347

THY ANOTIER, YES OR 310

297.143

337.439 
TRANSITION ANALYSIS: 8-7 YEAR TREND

DISTRICT \#2, GREEN ISLAND (MIXED STRATUM)

GRADES $4-5 ; 5-6$

$20: 45$ FRB 24 RER $2 \ldots$

FI:TPLE LIUEAR REGREASIOH VITH RESIDUALS

A.: COUFIDFUCS LIU:ITS

$? 7$

VEUES $Y, x$ BOR ORSERVATIOHS

$2 \frac{1}{2}$

$\because: 50,2: 2$

2205,255

4

$: 205,234$

5
2198,234

6

$? 315,312$

$? 304,385$

\begin{tabular}{|c|c|}
\hline & $\because F A n$ \\
\hline $2 O P V A R Y$ & 226.857 \\
\hline Fin VAR X & 206.571 \\
\hline BPo $=780 \%$ & 33.5777 \\
\hline 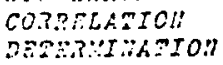 & $\begin{array}{l}.800117 \\
.6180186\end{array}$ \\
\hline Y IATERCRP A & 30.2175 \\
\hline$E L O P: B$ & .715154 \\
\hline$:$ & $2: 0$ \\
\hline 2 & $\$ 50$ \\
\hline 3 & 205 \\
\hline 4 & 205 \\
\hline 5 & 198 \\
\hline 6 & 316 \\
\hline & \\
\hline
\end{tabular}

STABDARD DEVIATEOH

60.3333

9 BROCHI CONFIDERCF LIUTTE

Loter?

$-132.575$ 9. $869795-02$ pentertan

167.805

187.830

238.532

239.321
20.3 .5614

259.340

311.552

UAYE 95 PERCEHT COHEIDEUCE IUTERVAL

PREDICIIOUS, YES O.? 170

? $2=5$

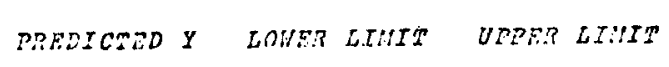

$\min x=$

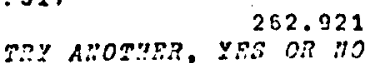

213.393

312.449

$-34.32 .13$

$-5.55356$

$-7.55180$

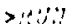

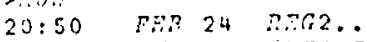

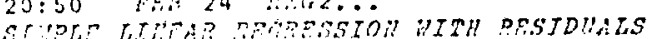

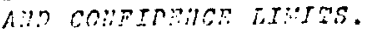

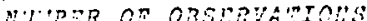

VCLUES Y,X FOR ORERNHATONS

$? 172.130$

265.210

$? 111,250$

$?+204,205$

5

$? 114.205$

6

?123,196

7,310

131,316

Man

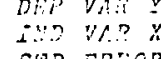

125.714

203.143

42.0682
51.5134

conpriston $40.435, ?$

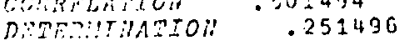

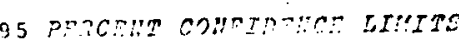

r.o:?:?

$y$ THTERCHPT A

39.9482

$-137.881$

$-408521$

berstcment

113.510

126.070

101.150

123.994

123.394

121.08

170.097

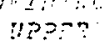

215.578

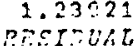

50.3200

$-6: .07 .33$

9.35031

$-15.93 .36$

$-9.935:$

1.91353

3203.12

57.0297

MThe

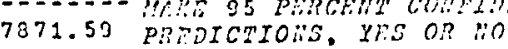

?YRS

PrintCing $y$

LONER LII:IT

UPDE:? LI:UIm

$\operatorname{mai} x=$

7263

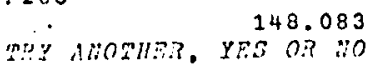

28.8135

207.354

?t:

(1)

3720.50

97.2527

92.8740

3.05274

3.65274

8176.23

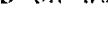

3.10 
TRANSITION ANALYSIS: $8-7$ YEAR TREND

DISTRICT $\# 2$, GREEN ISLAND (MIXED STRATUM)

\section{GRADES $6-7 ; 7-8$}

$20: 54$ FE? 24 Rra $2 \ldots$

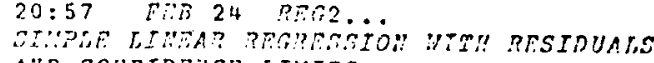

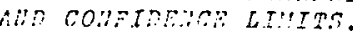

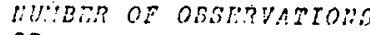

$? 7$

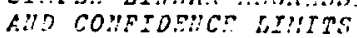

??

VILIISS Y.X FO? OSSERVITIOUS

YALVES Y.Y FOR ORSFRYATEONS

$? 161.149$

2

$?: \leq 5.104$

$? 215.172$

3

$? 322,11:$

2323.104

5

7

$? 149,155$

3

2149.216

2270.213

$\div 312,322$

$? 273,318$

$? 282.274$

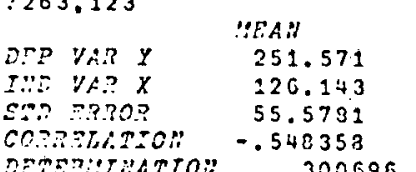

STAMTARD DTVIATIOI

43.4413

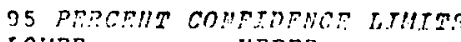
Low? ypes?

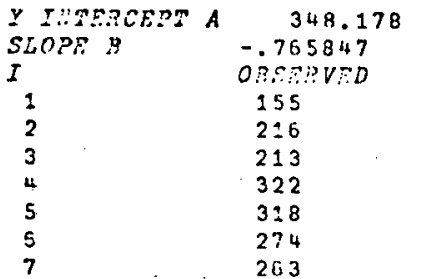

$170.375 \quad 525.370$

$-2.13870 \quad 577004$

PrerteTEn

139.603

RUTDUIA

$-144.5033$

$-.451893$

$-85.3975$

49.8315

13.1290

268.529

253.970

9.02153

YAYF 95 PERCË̈T CONETDENCE THMTRVA

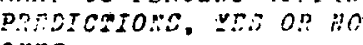

?:S

$\min \sin =$

PREDICMED Y LONER EIUYT UPPE? LTHIT

$? 148$

RP. AVOTHER: YFE OR NO

173.364

296.300
DEP VAR $y$

Inn $V A R X$

chom

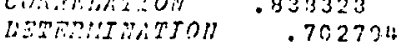

H.SA.H

229.714
235.286

43.1956

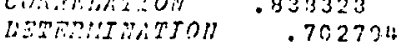

\begin{tabular}{|c|c|c|}
\hline & $\begin{array}{l}\text { Y TUTEROEPT A } \\
\text { SLOPE B } \\
\text { I. }\end{array}$ & $\begin{array}{r}20.5145 \\
.854705 \\
0320 ? V E ?\end{array}$ \\
\hline SOIISRED & 1 & 151 \\
\hline $\begin{array}{r}230.45 \\
1230\end{array}$ & 2 & 149 \\
\hline 204207 & 3 & 149 \\
\hline 7292.73 & $\begin{array}{l}4 \\
.5\end{array}$ & $\begin{array}{l}275 \\
312\end{array}$ \\
\hline 3461.14 & 5 & 279. \\
\hline 2447.33 & 7 & 282 \\
\hline
\end{tabular}

STAIRAPD DEVIATICH

72.8325
71.4370

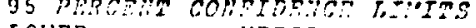

Lop?

131. 3:97

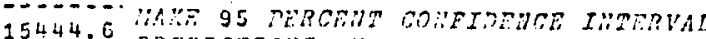

rine yos on in

pyos

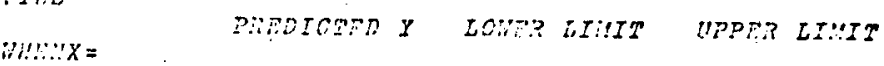

3235

miny noming y.0.470

$?: 10$
127.578

D.?

155.205

101.034

213.231

210.667

303.829

300.111

262.804

$$
\begin{aligned}
& 10.4 .905 \\
& 1.40377
\end{aligned}
$$

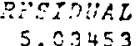

$$
\begin{aligned}
& -12.0537 \\
& \begin{array}{r}
-12.0237 \\
-64.2307 \\
5.5 .3334
\end{array} \\
& \begin{array}{r}
-64.2307 \\
r 5.3334
\end{array} \\
& \text { 3.170 } \\
& -21.4175 \\
& 19.1964
\end{aligned}
$$

187.203

271.737 
TRANSITION ANALYSIS: 8-7 YEAR TREND DISTRICT 非, GREEN ISLAND (MIXED STRATUM) GRADES 8-9

21:00 FR 24 REG?

AID COUFTOEIC OTIITO

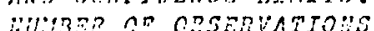

?o

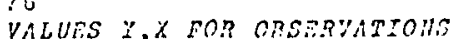

$? 144,161$

221.149

321.149

227,143

4

291.27

311,312

320,273

DEP VAR: ?

STO BRroi

conomiatio

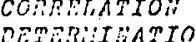

235.667

235.66

156.57111
75.6809

44.0552

80415

Y IMTERCEPTA

10.5612

$\sin 2$

YLOPE B

C.5.:?"

$-155.773$

295338
PREPTCOED

174,552

162.329

162.329

291.69.

$\begin{array}{ll}291 & 291.699 \\ 311 & 320.357 \\ 320 & 2916.744\end{array}$

$\begin{array}{ll}291 & 291.699 \\ 311 & 320.357 \\ 320 & 2916.744\end{array}$

intove

MARE 95 PRRCEN COMP

PREDICIOHS, YRS O? \#\%

?YES

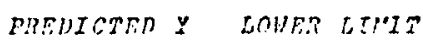

TFIDRCE UIUITS UPpor

177.825

1.74126

$-30.5521$

$-41.3291$

-41.0709
-.60309

$-000395$

$-17.3571$

25.2559

sovasen

933.429

1708.10
4182.32

.473875

301.271

637.860

7763.45

HIIIXX
$? 229$

243.815

TRY ALOTHER, YRS OR VO

133.554

UPיP LI:IT

2010

•


TRANSITION ANALYSIS: 8-7 YEAR TREND

DISTRICT 非, RIVERSIDE-DIAS (MIXED STRATUM)

BIRTH-GRADE 1 ; GRADES $1-2$

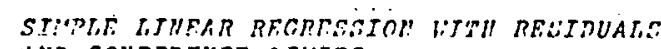
AIT COHFIDERCE LTUYTS.

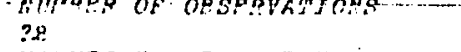

VALUTS Y.X POP ORSFRVATJOLS

2279.224

$? 27$

$? ? 71.222$

?3 312,250

$? 273,226$

$? 273,226$

5

2.230 .242

2295,263

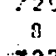

$\div 275.254$

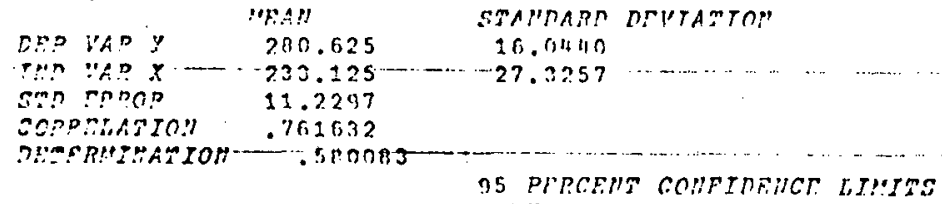

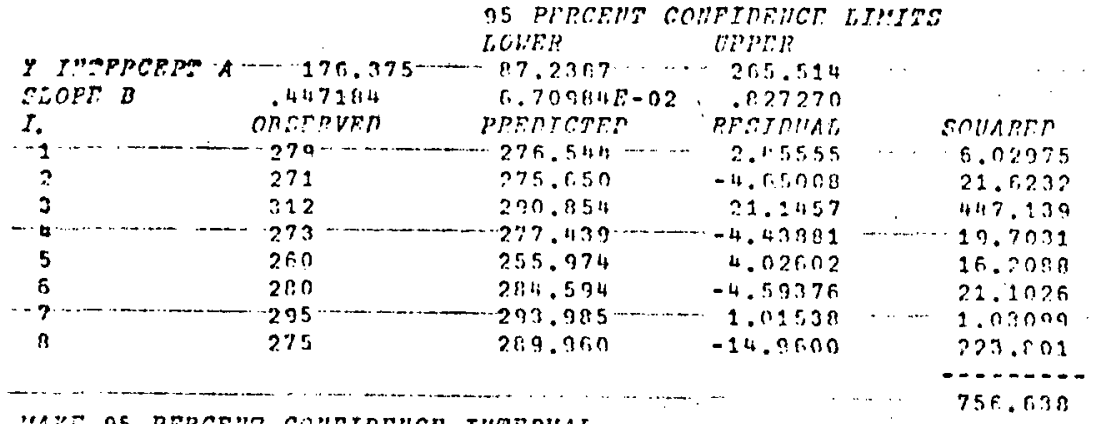

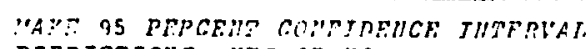

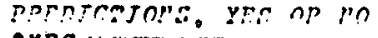$$
\text { PYrs }
$$

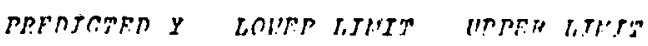

prong=

$\begin{array}{llll}291.016 & 271.295 & 290.737\end{array}$

On: 27 FEP 25 PECS.

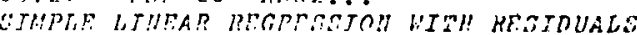

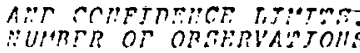

?7

VALUES Y $X$ FOR OPAYPLATYOMG.

$\frac{1}{2020279}$

$?$

3

4.2840 .073

$? 25,3,260$

is.

$\frac{10.000}{3}$

2308.295

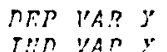

$\lim$ Gin

CORRTHAZTOR"

DEREREIUATTOH

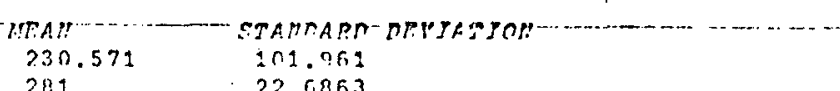

$191.606 \cdots$

$935,00.02$

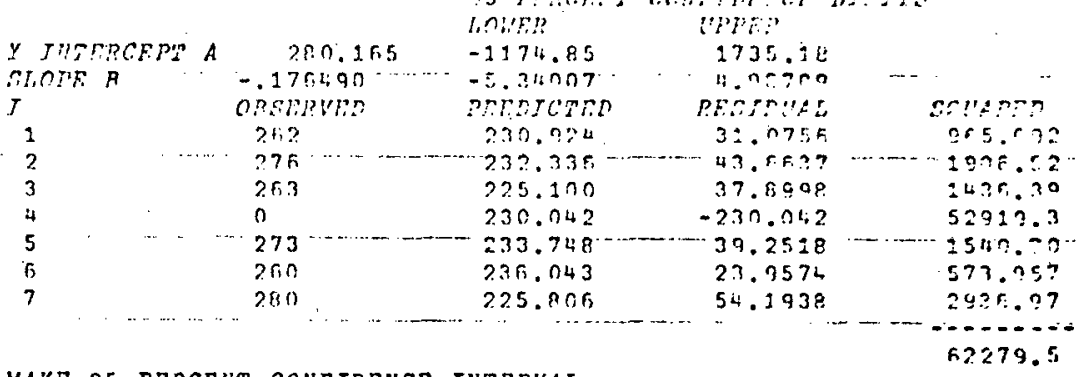

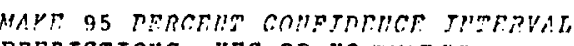

prontertatrs.

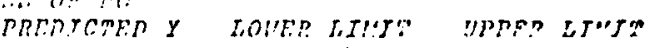

$m ! n y=$

$? 201$

230.571

122.119

339.024

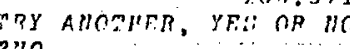

?mo

TPY AMOTPER, YPS OP TO

(19.20


TRANSITION ANALYSIS: 8-7 YEAR TREND

DISTRICT \#4, RIVERSIDE-DIAS (MIXED STRATUM)

GRADES $2-3 ; 3-4$

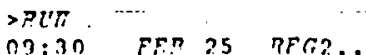

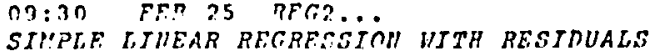

SIVPLLF LIIIEAR REGRECS

MUUAR OF OBSEPVATIOI'S

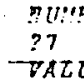

270.278

$? 270,262$

3

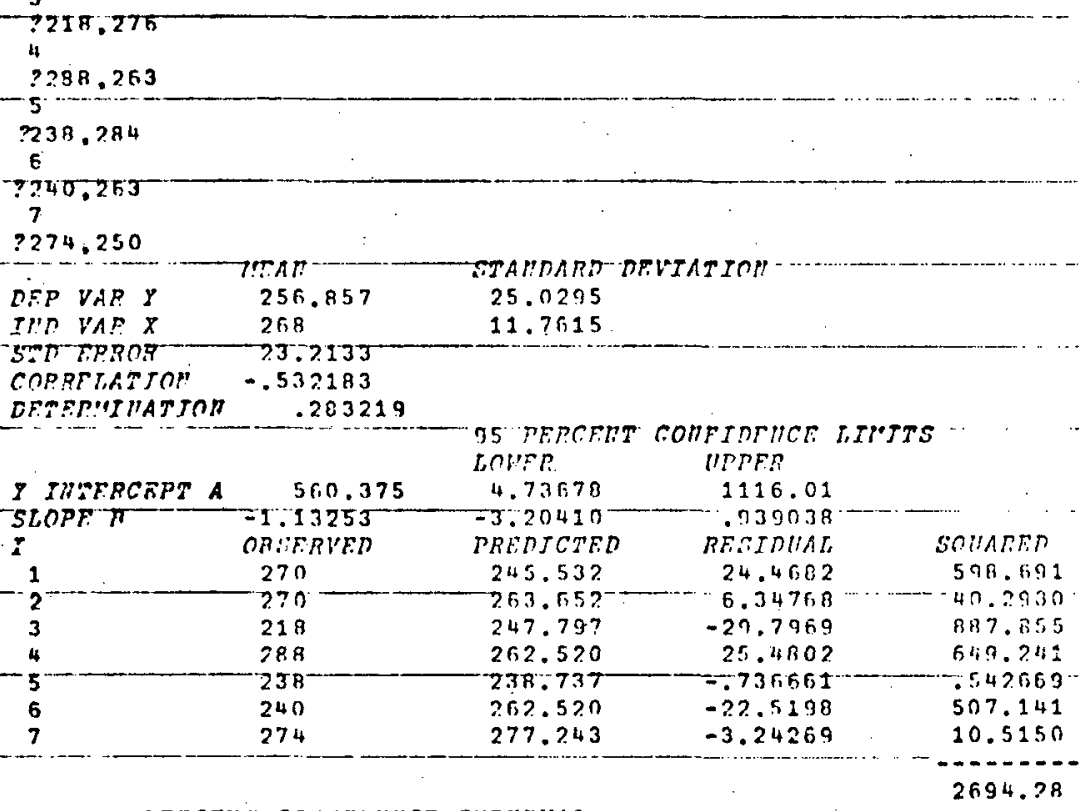

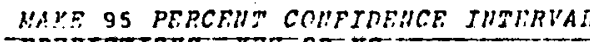

PRETICTTONS, YET OP MT

PYFS

GPF $\cap 7 \mathrm{~T}=$

PRENTCTFA $Y$ LOUFR LIHTT

UPPER LITHT

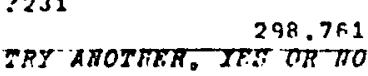

218.862

378.659

2694.28

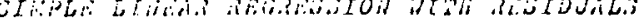

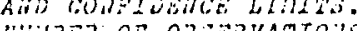

$? 7$

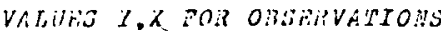

$? 247,289$

$? 247,270$

$? 231,270$

2030.218

.230 .218

$? 282.233$

$? 245,238$

$? 253.240$

iRn

DEF VIR $Y$
IiA VAR $X$

Sint) $\operatorname{Ris} 30.3$

DeTibitianton $\quad .205301$

$247.35 \%$

259
16.2735

10.2735
.515050

STAWDARE DEVTATEDI

17.31139

27.2825

35 PFRCEUT COHPI TEUCF LIUTAS

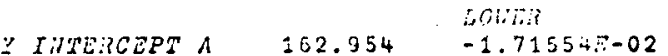

TLOP: $162.954 \quad-1.71554 .02 \quad 325.325$ $-230452 \quad \therefore 354073$

$257.0 .31 \quad-10.0514$

$257.131-10.0514$

251.403

234.417

$-20.4531$

$-20.4531$

4.4035

257.364

$\begin{array}{lll}202 & 257.364 & 24.6354 \\ 2.5 & 240.973 & 4.02587 \\ 253 & 241.629 & 19.3712\end{array}$

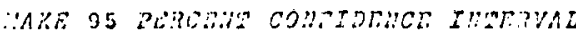

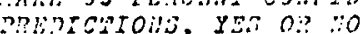

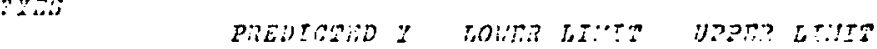

S4:1.?2:?

114.367

49.7337

4.7033

600.351

ic.2157

122.325

2324.34

arisitis=

5293

200.970

T.RY AHOSIRA, YES O:? 110

231.343

230.536 
TRANSITION ANALYSIS: 8-7 YEAR TREND DISTRICT 非, RIVERSIDE-DIAS (MIXED STRATUM)

17:27 $\because 1309 \quad \therefore-12 \ldots$

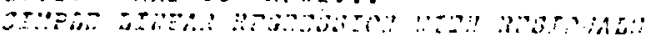

$\therefore$ COABTDE

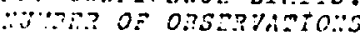

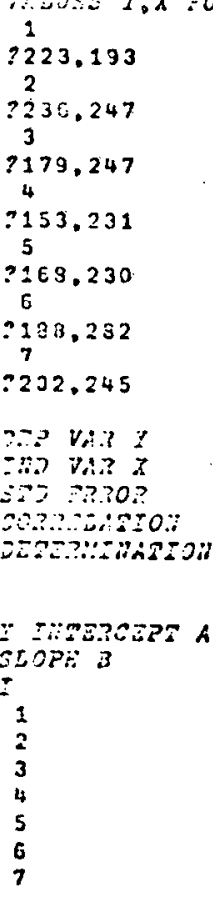

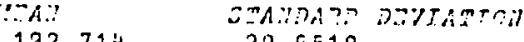

$\begin{array}{ll}132.714 & 23.6519 \\ 233.286 & 20.6373\end{array}$

32.0535

$-.150707$

$2.38525 \%=0$

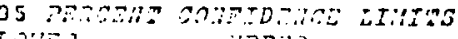

235.462

$-873045$

223

236

173

153

163

202

532.774

$-1.43351 \quad 1.0 .322$

1......

131.336

131.330

194.134

132.033

22.0175

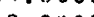

4.0702

$-20.3731$

2.31643

10.3065

GRADES $4-5 ; 5-6$

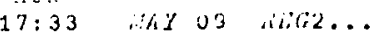

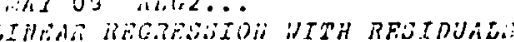$$
\text { Mini }
$$

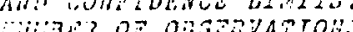

$? 7$

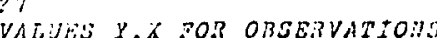

$\frac{1}{1356.130}$

213

$? 131,223$

$?+28,230$

$? 1+3.173$

5

$? 132,153$

$? 14.6,168$

2157,138

DiR $Y A$ I Y

Iii) $\forall A$ :

contrietstoi

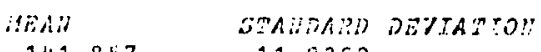

141.857
$1,2.143$

11.9353
23.4812

12.11363

$9.53167 E-02$

ARE 35 PERCEUT COIT TAEHCE IHTERVAL

PREOESTOATS, YES O:P iOO

\section{Widitid= \\ PREDICTED Y LOLER LIUTS \\ UPPER LTIIT}

$? 201$

$147.300 \quad 230.310$

237 A.2OLHER, ZES 0.3170

0.310

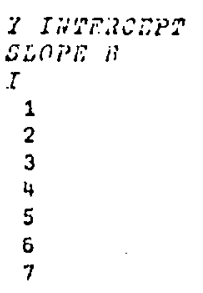

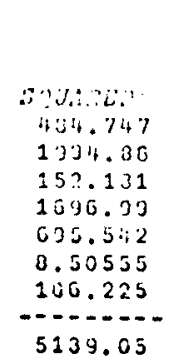

5139.05

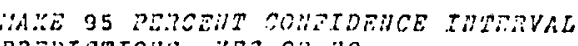

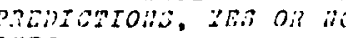

$3 Y 25$

$\because \pi: 1 \%=$

2139

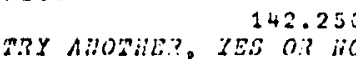

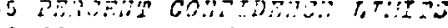

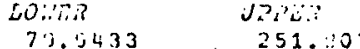

$-.507733$

ind

141.125
130.000

136.375

143.500

146.750

144.87

142.37

251.007
317723

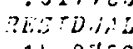

$14.8 \%=0$
-7.00000

$-2.37500$

$-.500003$

$-14.750 .2$

1.12530

14.6250

रㅏ. 223.250

223.250
43.0000

0.2430

.25002 .3

217.502

1.26503

213.831

773.375

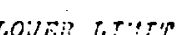

130.035

43:38 2.285

54.415 
TRANSITION ANALYSIS: 8-7 YEAR TREND

DISTRICT 非, RIVERSIDE-DIAS (MIXED STRATUM)

GRADES $6-7 ; 7-8$

$>P::$ :

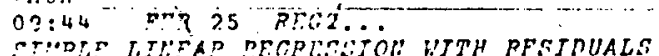

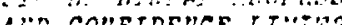

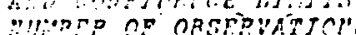

$? 7$

WLUF: Y Y FOR ORCFPVAMTOMS

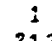

$?: 32,: 87$

$? 153,156$

$\because 33,131$

$? 213.228$

251,243

2143,132

7,130

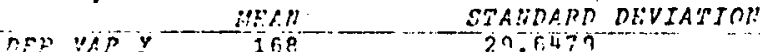

$\because \because 2 \pi x$ 14r.143 20.5542

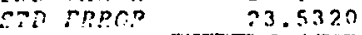

COBPRAATCH $=0.09213$

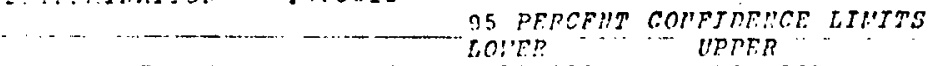

y tomatrotst

SLOPF R -.994:39 - -2.29580

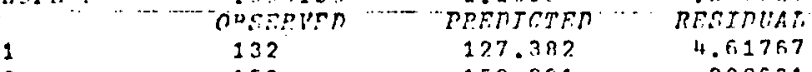

$\begin{array}{rrrr}2 & 158 & 158.201 & -900631 \\ 3 & 193 & 283.054 & 3901 \% 90 \\ 4 & 218 & 186.037 & 31.9535\end{array}$

$218 \quad 18.6 .037$

151
171.124
182.060
178

$176 \quad 168.142$

$-20.1244$

-34.0500
7.85798

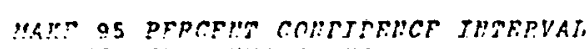

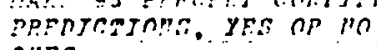

ars

ment:

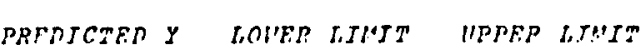

$?: 42$

172.119

148.716

135.521

noy AHORHER, YLE OPP
$>R(m$

$0 ?: 117$ FFS $25 \quad R F C 2$.

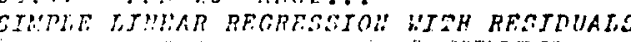

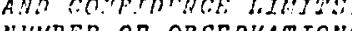

"ARIUES Y.X TOR OPSEPUATSOHS

$? 2314.131$

2

292,132

$? 117,150$

$? 189,193$

$? \frac{0}{203}, 218$

ri.

?5? 7.151

264.148

DRP VAR Y
IVP VAR
OPS

IIS TEROR

CORRELAETOH

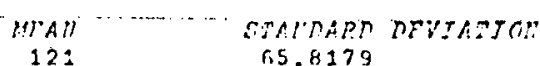
6.5 .8179

$182.571 \quad 32.3772$

Conntirent $-4.21452 F^{\circ}-02$

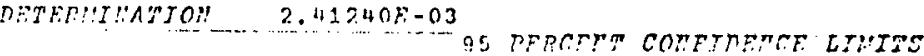

\begin{tabular}{|c|c|c|c|c|}
\hline Y IMTRCRPT A & 137.132 & $\begin{array}{l}Z C F^{p} \\
-246.495\end{array}$ & $\begin{array}{l}\text { UPPYR } \\
520.760\end{array}$ & \\
\hline $\begin{array}{l}Y \text { IMERCEPT A } \\
\text { SLOP! } P\end{array}$ & $=9.9845 \%=2$ & $\begin{array}{l}-246.495 \\
-2.23436\end{array}$ & $\begin{array}{r}520.760 \\
2.234,57\end{array}$ & -1 \\
\hline .7 & n n:rn!rn & rpongons & prasnuth & ECUADES \\
\hline 1 & 234 & 124.052 & 109.048 & 23000.5 \\
\hline 2 & ? & 123.753 & -31.9526 & $90.9 \% 77$ \\
\hline 3 & 117 & 121.357 & -4.35850 & $9 n .3799$ \\
\hline 4 & $28 \Omega$ & 117.562 & 70.1390 & 4010.34 \\
\hline 5 & 93 & 115.366 & -22.3659 & 500.232 \\
\hline 6 & 59 & 122.056 & -63.0555 & 3976.00 \\
\hline 7 & ril; & 122.355 & -58.3550 & $34 \cap 5.32$ \\
\hline & & & & $2502=.2$ \\
\hline
\end{tabular}

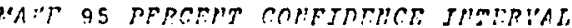
PPROICTSOHS, YF:" $O R$ " $M O$

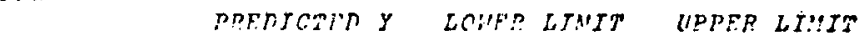

0172

mpy cuotura, yos 129.959

45.8664

194.051 
TRANSITION ANALYSIS: 8-7 YEAR TREND

DISTRICT \#4, RIVERSIDE-DIAS (MIXED STRATUM) GRADES 8-9

3 RUN
$03: 50$

$03: 50$ TER 25 Rroz.

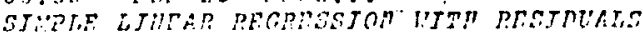

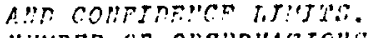

Al:?

HFS $y, x$ rOp ODETIVATROHS

1

?1:1. 234

$? 33.92$

$?: 24.117$

$4,5.180$

5

258.59

Dren $\operatorname{lng} \mathrm{y}$

UTA"

STAMARD DEVIATICI"

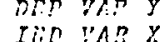

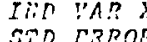

8.7 .5000

38.2034
$6,0.0356$

37,7597

Corretiston - .46.7415

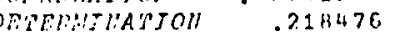

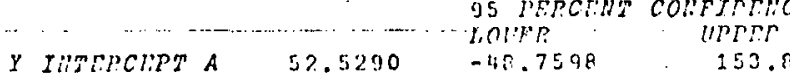

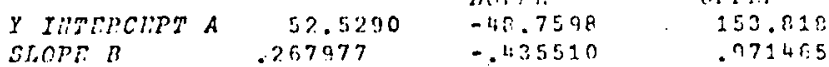

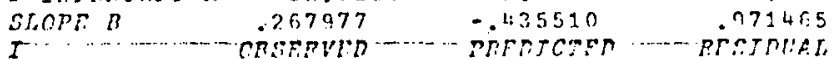

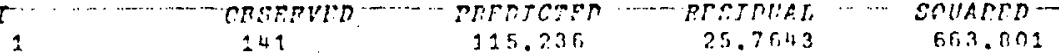

25. 25.173 65.3. Bn

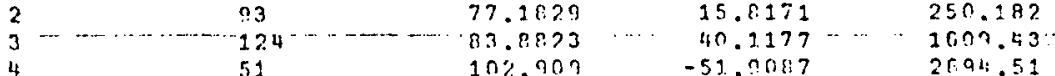

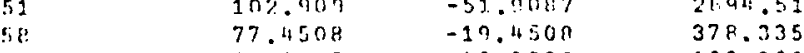

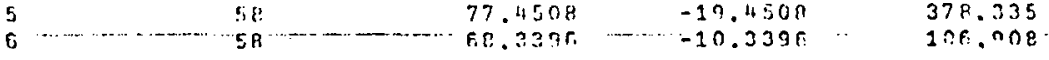

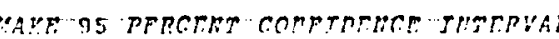

5703.17

PREDTCTIOIS YEG CP Ho

pyrs

WIIIIY

?1?0

moy chothen y 84 .

?llo

128.112 
APPENDIX F5

TRANSITION ANALYSIS

SIX - FIVE YEAR TREND

SEVEN DISTRICTS 
TRANSITION ANALYSIS: 6-5 YEAR TREND

DISTRICT \#涪, HOPEWELL (STRATUM 非I)

BIRTH-GRADE 1; GRADES 1-2

$23-31$ fei) 22 reg $2 \ldots$

simple lincar refression with residuals

muiner of obsarvations

$1: 0$

Whes $y, x$ for observations

1

:213,2:1

1:237,203

$1: 3,37,209$

4

9312,227

11285,155

60.150

1!255,252

deg var $y$

ind var :

correlation

deterifination $2.38686 e_{-02}$

mean standard deviation

$275.333, \quad 37.3536$
$210.067 \quad: \quad 31.5257$

i. 1521

y intercept.a 313.741 low

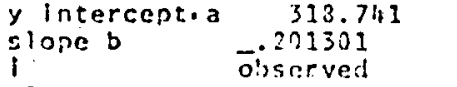

5 percent confidence $11 \mathrm{mlts}$
1

2.33

2.13
20.7
$3: 7$

iis

206

lowrer
25.2254

$-1.32135$

oronicterd

275.206
275.050

278.609

7.7 .045

$2.77 .3 \pm 3$

6

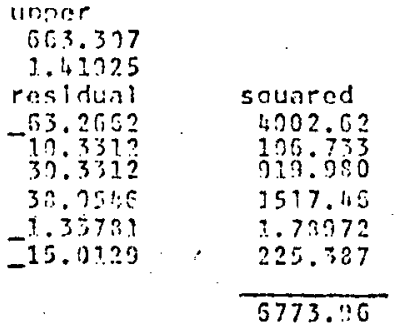

matic 35 percent consil jence interval predictions, yes cir no

iyes

$$
\text { predicted y lower limic upoer limit }
$$

19214

$275.662 \quad 229.713 \cdot 322.612$

tr: mnother, yes or no i:no

20730 foll 22 rages...

iriple linear rciression with residuals

and eonflatence itrites.

15

values $y, x$ for observations

$4: 375,23=$

$\stackrel{2}{2} 20,237$

3301,307

$\int_{1}, 301,307$

$! 283,312$

$1: 244,236$

dep var y

ind var $x$

correlintion

deteraination

$\operatorname{menn} 24: 5,300$

245.300

22.1144

.032433

.351382

$\begin{array}{ll}y \text { intercent a } & -77.5589 \\ \text { slope b } & 1.25774 \\ i & \text { observed } \\ 1 & 373 \\ 2 & 226 \\ 3 & 301 \\ 4 & 283 \\ 5 & 24 !\end{array}$

95 percent confidence linlts

lover wiper

-322.230
-.255733

pre!licted

267.549

252.704

28.473

251.554

male 95 percont confidence Interval

predlczions, yes or no

lyes

whenx:"

prodicted y lover linte

$2.03572 \frac{1}{2}$

residual

$-26.7945$

1.52695

7.55372

55.5107
713.128

5.?. 112

57.0525

$11: 67.24$

1276

try another yas or

t? no 
TRANSITION ANALYSIS: 6-5 YEAR TREND

DISTRICT \#9, HOPEWELL (STRATUM 非I)

GRADES $2-3 ; 3-4$

>run

$20^{-41}$ feb 22 reg2..

simple linear repression wth residuals

any confluence ilmits.

:

vialues $y, x$ for observations

1

$1: 220,205$

: 2210,275

$1: 224,220$

1202,331

302,391

den vary

ind var $x$

stu error

deterrination

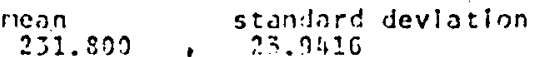

squared

23.1097

159.253

0.02600
3.13977

206.529

rake 25 percent confidence interval predlctions, yes or no

?:yes

predlcted $y$

vahenx:

(1:240

try another, yes or 00 $\operatorname{lino}$ lover limit unoor llmit

220.846

244.481

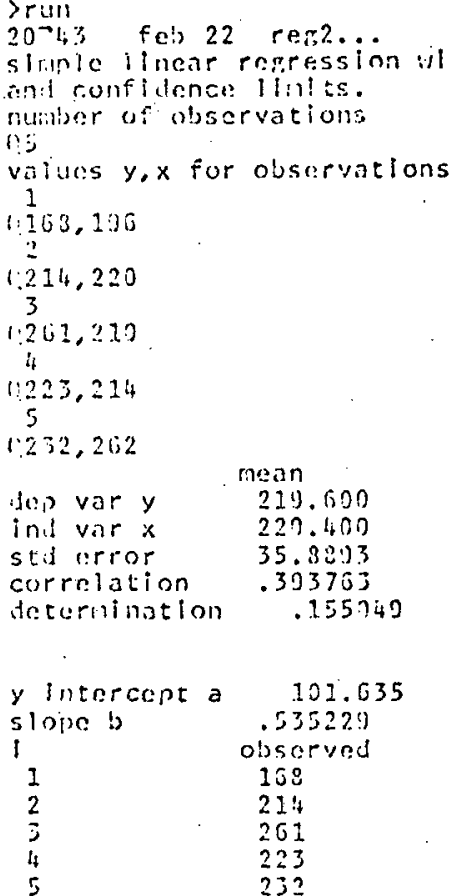

1

$\frac{1}{2}$

observec
102
214

214
261
223
230

d deviation 24.8757

95 percent confldence linlts

lover

-406.314
-9.76918

Trelicted

206.2109

257.326
$23 ! .034$

216.175

$241.36,6$

upor $612: 25$

2. 23.35:4

residinal

$-5,3053$

40.3054

46.3654
0.825147

G. 36555

squared

23.0530

2205.34

40.5370

$97.32 ? 0$

3.54 .23

make 35 percent confidence interval

predictions, yes or no

liyes

whenx:;

$1 ! 233$

prodleted y lower limit unper limit.

220.344

167.851

285.036 
TRANSITION ANALYSIS : 6-5 YEAR TREND

DISTRICT \#9, HOPEWELL (STRATUM \#I)

GRADES $4-5 ; 5-6$

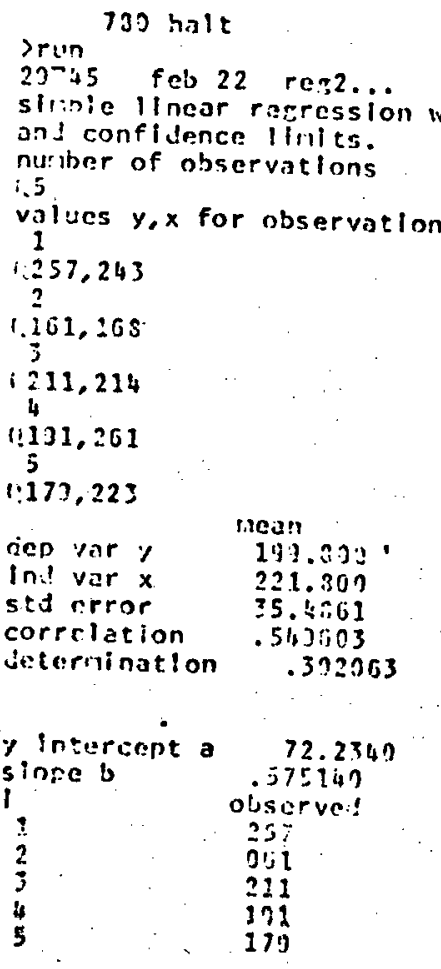

make 95 percent corifl dence Interval pretllcilons, yes or no

Whinn:

predlicted y

lover llmit

try another, yes or no

151.267

253.162

lower unner stondard Jeviation

35.1525

35 nercent conflidence llinlts

$-1.03030$

profleted

211.403

225. 31"

22.3145

230.131

uni?..220

2.3124

resletini

$-7.35749$

$-31.3455$

$-21.4702$

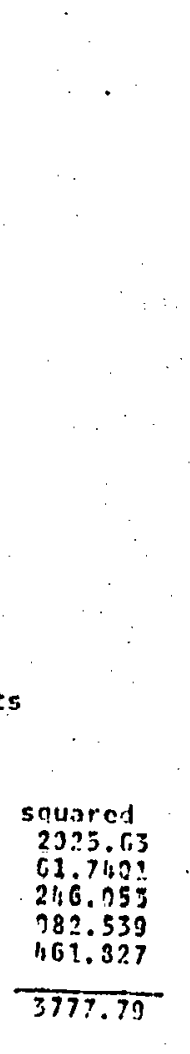

103.

stanianrd devlation 10. 5ny

43.1345 moke 35 percent ennflidence interval
pre.tictions, yes or no

nyes

95 bercent conflinnce linlts

loiner

107.773
$.02147 ? 3$

prodlcted

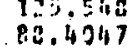

110.482

116.1182

101.358

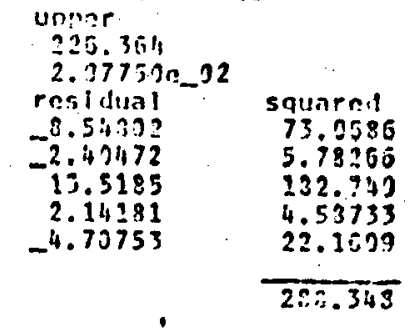

prolloted y inver limle

unner linlt

Whenx

104.490

try another, yes or no

97.0132

118.368 


\section{TRANSITION ANALYSIS: 6-5 YEAR TREND \\ DISTRICT \#9, HOPEWELL (STRATUM \#I) \\ GRADES $6-7 ; 7-8$}

srun

$22^{-51}$ fe!s 22 reg2..

siaple llnear regression with resiluals

an I confidence limits.

nulver of observations

values $y, x$ for observatlons

1

$1: 122,125$

$1: 245,117$

$1: 265.36$

1264.133

$1.264,13]$

1.154 .134

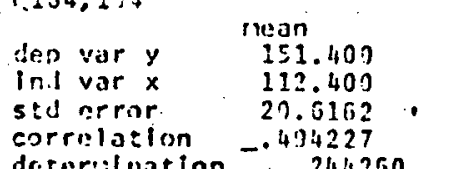

decteriluation -.0214250

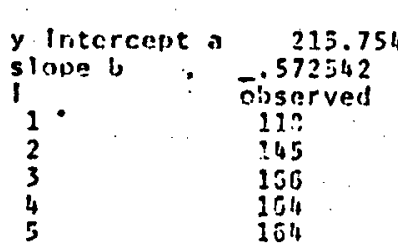

35 percent ennflience limits

lover: upper

$5.73775 \quad 425.770$

$-2.42203$

presticend resirlinil

$144.136 \quad-25.1360$

14.701 -

$165.5150 \quad-325113$

$155.253 \quad 7.73065$

mate 35 percent confldence interval

prodlctlons, yes or no

?yes

itronx?

rerefleted

lower linit

upper linit

19.

try another, yes or no

123.010

189.439

tho
$20^{-53}$

sinple linear repression w! th residuals

and collifidence litits.

number of observations

$1: 5$

values $y, x$ for observations

1

$\ln 9.172$

2

$1: 115.113$

13257,143

$1 ! 134,200$

$\frac{134,100}{5}$

$1 ! 133,164$

Jen var $y$ liean standard deviation

latir $x \quad 25.200 \quad 25140$

Inil var $x$
stil crror 22.0227

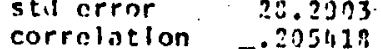

detern!nation ${ }^{-2}$ 4.21064e_02

35 percent conflidence linlts

y Intercept a

$4.21304 e^{2} 02$

snumred
685.795
14.7951
265341
514.235
60.69142

sione

$-2 \frac{160.926}{335}$

$-154.555$

obsierve:

$-2.27730$

prelleted

120.7 .03

83

116
157

157

- 127.373

127.069

122.104

unper

$47 \pi .207$

residual

$-31.763: 5$

$-17.372 ?$

i]. 8350

7.35356

1035.5

$301.3: 2$
205.022

149.091

54.2356

$\overline{1275.08}$

tiblec 25 percent conflidence Interval

predlctions, yes or no

?yes

whenx:

prellicted y

lover limit

unper llirit

1156

124.499

try another, yes or no

83.7772

$\$ 55.222$ 
TRANSITION ANALYSIS: 6-5 YEAR TREND

DISTRICT 非, HOPEWELL (STRATUM \#I)

GRADES $8-9$

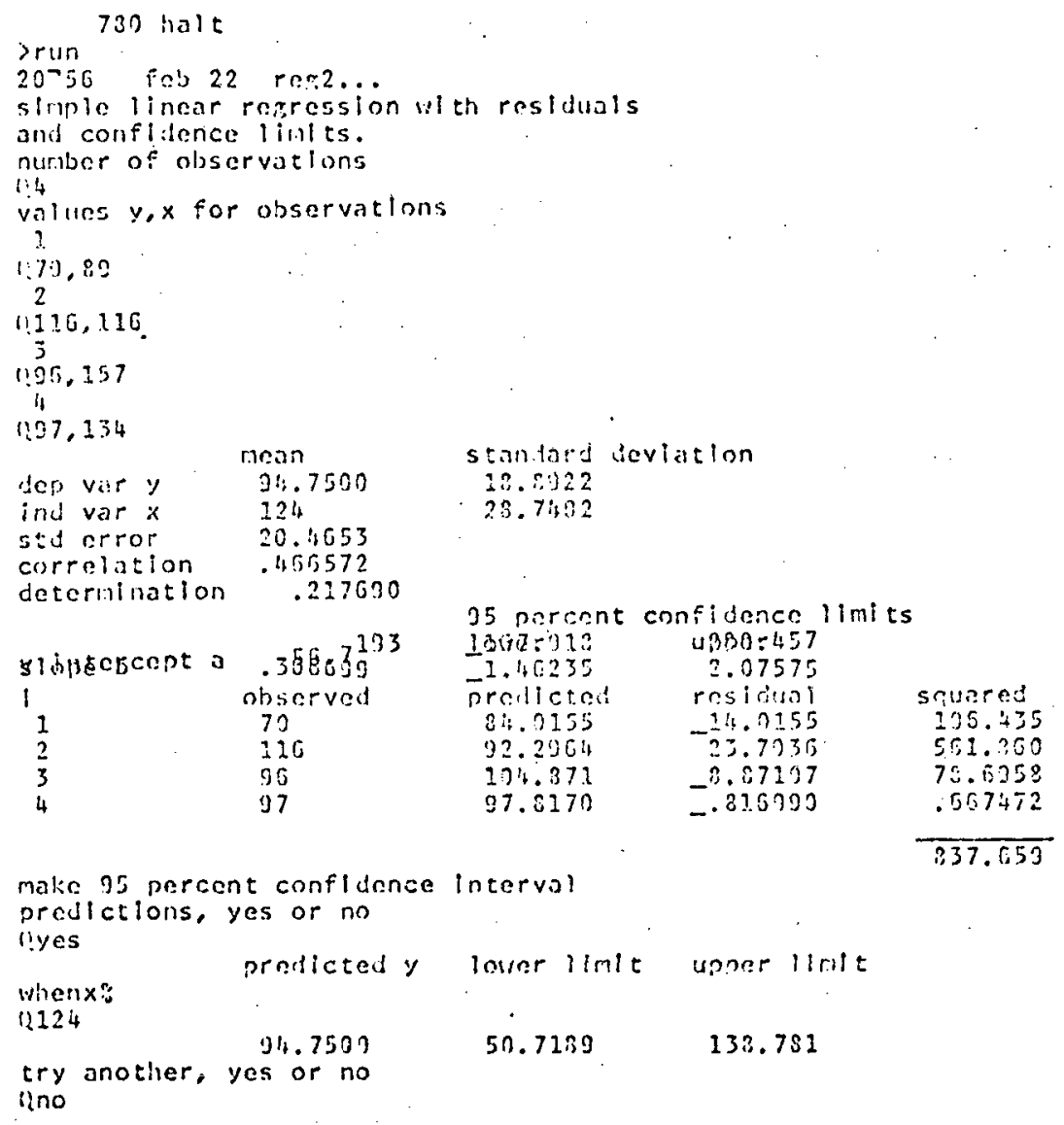


TRANSITION ANALYSIS: 6-5 YEAR TREND DISTRICT \#8, SANDY BAY (STRATUM 非I) BIRTH-GRADE 1; GRADES 1-2$$
\text { >UUA!" KESIL }
$$$$
>?: .8
$$$$
21: 01 \quad 583 \quad 22 \quad \pi 562
$$

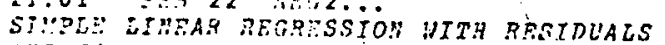

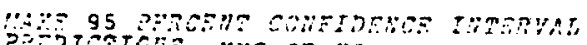

ons, yits o? :0

?I:E

$x: 7 x=$

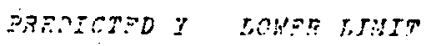
$?: 05$

T35 ANOMER, yes on ro

66.3203

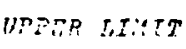
.70

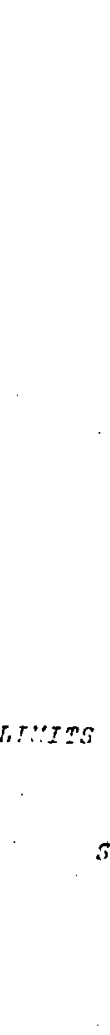

123.321 
TRANSITION ANALYSIS: 6-5 YEAR TREND

DISTRICT 非8, SANDY BAY (STRATUM 非I)

GRADES 2-3; 3-4

$>0,31:$

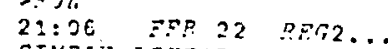

COPR LTMAR RETREJSTOH WITH RESTOVALS

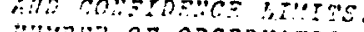

$? 5$

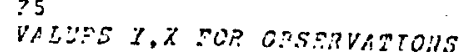

$=11.53$

5

$? 77.71$

$\stackrel{4}{0.93,90}$

5

$? \div 11.105$

EDP WAP Y

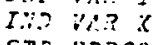

ccosediston

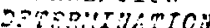

"SAAl

91.2000

7.33524

352944

I.

12.5379
15.1517

y IMTacost $A$ .743293

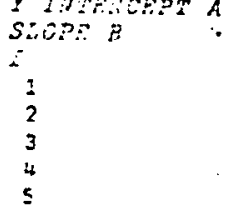

$$
\begin{array}{r}
5 \\
055 \\
85 \\
89 \\
77 \\
93
\end{array}
$$

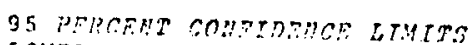

LOWF" UIPP?

$-29.5590 \quad 35.6505$

$-5.32650 F-02,1.301004$

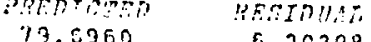

$\begin{array}{lr}19.5360 & 0.72398 \\ 31.7351 & -2.73507\end{array}$

$\begin{array}{ll}91.7351 & -2.73507 \\ 31.0337 & -4.03309\end{array}$

$32.0337-4.03369$

$9.7011 \quad-5.76110$

7.22538

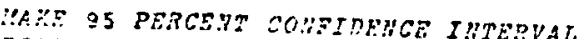

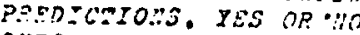

?Y:S

$\because 727 x=$

PREDEGTED Y LOHER LTMTT UPPER LTMIT

395

MPY AHOMUER, YRT 97.7546 $3: 10$
35.1430

110.366

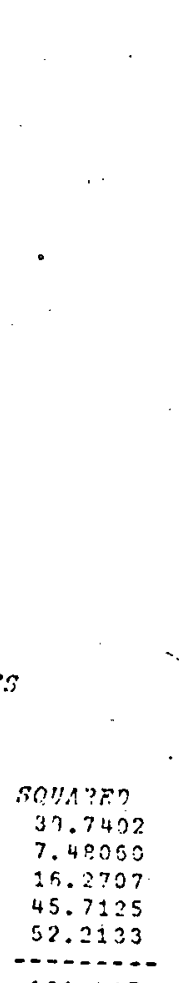$$
\ldots \ldots .
$$$$
\text { 21:03 REB } 22 \text { RER R. }
$$

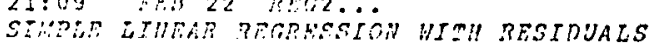

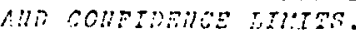

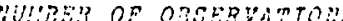$$
\text { VILUFY Y.X FOR OBAF?VAZTONS }
$$$$
\text { ? } 73.134
$$$$
\begin{aligned}
& 2 \\
& ? 65.30
\end{aligned}
$$$$
? 118.89
$$$$
? 73,77
$$$$
\begin{aligned}
& 5 \\
& .75,93
\end{aligned}
$$

Lep $\min y$

$M: A H$

STAIIARD DEVIATION

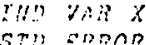

92.8000

20.7605

23.3721
$-2.174475-03$

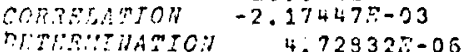

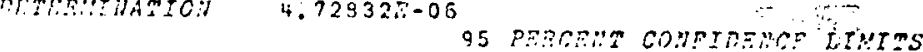

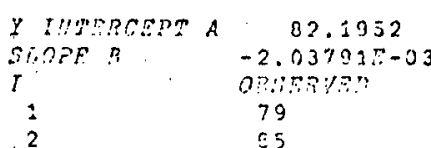

MARE 35 PERCENT CONPIDENCF IITERVAL RREICEIOLIS. TEA OR :O

?YSS

$\tan \theta X X=$

BREOTCTED Y LOHR LTHT UPPEP EIUTT

$? 90$

TRY AHOMHER, YES OR 910 
TRANSITION ANALYSIS: 6-5 YEAR TREND

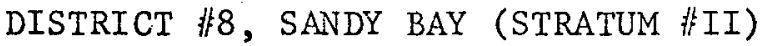

GRADES $4-5 ; 5-6$

$>R \cup R$

21:11 FER 22 REG2

STMPIE LINEAR REGRESSION WITH RESTPUALS

A.UN CONFIDEUCE LTUITS.

WU:"BER OF OBSERVATIOUS

? 5

VALUES Y,X FOR OBSERVATIOHS

$\stackrel{1}{? 66,57}$

$? 2$

3

$? 51.65$

$? 72.118$

5

245.73

DEP VARY $Y$ YAN

IIID VARX $X \quad 78.4000$

STD ERROR

COREFLATION

17.6983

.362666

DERERIITATION $\quad .131527$

I INTERCEPT A SLOPE B

I

2 .

3
4
5

5

44.2176 OBSSRVED

66
80

80
51
72

$$
\begin{aligned}
& 16.4469 \\
& 23.6390
\end{aligned}
$$

$-52.5064$

PREATCTED

58.6002

64.1514

60.0188

73.9921
STAIDARD DEVIATIOH 95 PERCENT CONFIDENCE LIMITS
LOWER UPFT?

62.6374

MARE 95 PERCENT COHEIDENCE INTERVAL PREDYCTIOHS, YES OR IIO

?YSS

HRENX =

PREDICTED Y LOWER LIHTT 382

64.9084

39.3607 UPPT.?

140.942

1.44349
$R T S T D U A L$

7.39979

21.8486
-9.61883

$-1.93213$

$-17.5374$

UPPER LIUIT

564857
54.7568
477.362
32.5218
3.36857
311.079
91297
939.688

$>R$ UI

$21: 14 \quad \operatorname{SER} 22 \quad R E C 2$

SIHPLE LIHEAR REGRESSTON WITH RESIDUALS

AND CONFIDENCE LIUITS

DUUBRR OP ORSERVATIOIS

? 5 ALUES Y,X FOR OBSERVATIONS

252.28

2

$? 61,66$

341,86

4

?33. 51

736,72

MEAN STALDARD DEVIATION

$\begin{array}{llll}\text { DEP VAR } Y & 44.6000 & 11.6748 \\ \text { IUID VAR X } & 60.6000 & 22.1314\end{array}$

STD ERROR $\quad 13.1404$

CORRFLATION -.223315

DETERMINATION 4.93697E-02

95 PEREEUT CONFAREUSR LTHTTS

IITTRCEPT A

LOWF?

$-3.48341$

$-1.05245$

PRENTCTED

OBSERVED

1

2

3

5

939.688

UAKE 95 PERCEUT CONETDEUCE TIITERVAL

PREDICTIONS, YTS OR NO

?YES

W1: $F$ : $X=$

PREDICTED Y LOHER EIMIT

265

TRY ANOTHER, YES OR NO

24.9260

63.2373 
TRANSITION ANALYSIS: 6-5 YEAR TREND

DISTRICT 非, SANDY BAY (STRATUM 非I)

GRADES 6-7; 7-8

$\rightarrow ?: \%$

790 IALT

$21: 25 \quad Z E E \quad 22 \quad \operatorname{RSQ2}$

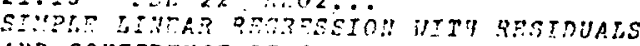

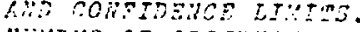

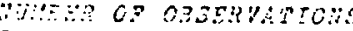

$? 5$

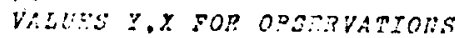

.? 31.47

:5ว.52

$? \underbrace{3}, E_{1}$

$? 23.42$

$\div 65.33$

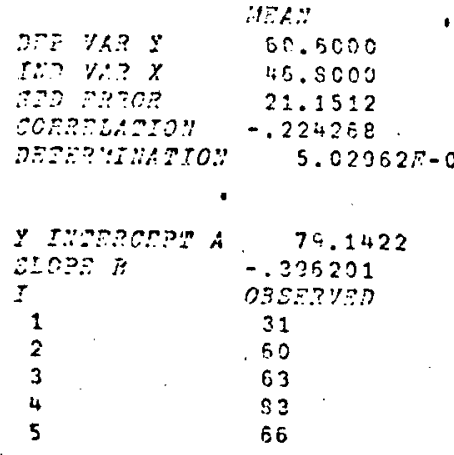

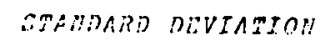

$\$ 3.7963$

0.5335

35 PERCFNT COUFIDTUCF LTUITS

COIP?

$-71.9736 \quad 230.134$

$-3.55303 \quad 2.76658$

pestrems

60.5208

58.5338

54.9739

62.8380

66.0675

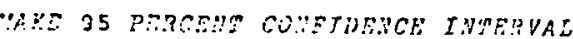

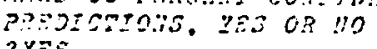

$m: m x=$

PRERICTED 7 LUWER LIHTT

$? 44$

TPY AIOTHER, YRS OR 0.7094

30.3346

93.0841

$-29,5208$

1.40025

8.02600

20.1020

$-6.75795 F-02$

$S 611.75 n$
871.475
2.13232
84.4175
404.032
$4.566938-03$
-2.5 .25
1342.12

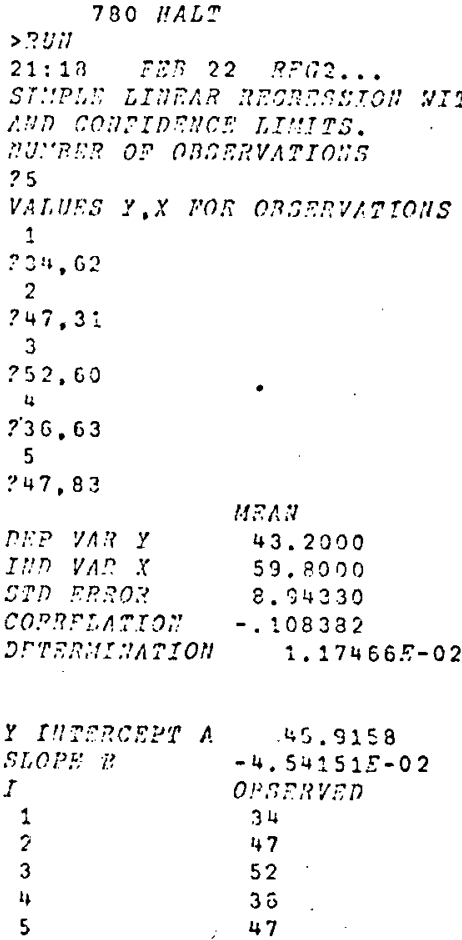

ETAMARD nEVIATIOH

7.73102

3.5930

IS PERCENT CONFTIRUAR LIUTTE

$-1.58: 35$

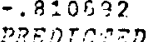

43.1001

144.5080

43.1903

43.0547

$42.21: 54$
IPS:
$33.4: 60$
712861

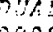
$-3.40003$
8. 80308
$-7.05467$
4.85363

SOUCSED

$25.81: \epsilon$

E. 2023

40.7584

1342.12

MAYE 95 PERCEHT COMEIDENCF TMMERVAL

FREDICTIONS. YRS OR 10

?.YES

H!! E:UX =

PRENICTEDY Y LOHES LIUIT UPPEP LIIIT

262

TRY ALOTHER, YRS OS 430

320 
TRANSITION ANALYSIS: 6-5 YEAR TREND DISTRICT 非 8 , SANDY BAY (STRATUM 非I)

GRADES 8-9

780 IIALT

$21: 21 \quad F R B \quad 22$ RFIR $2 \ldots$

STEFTE LENFAR RECRESSTCH WITH RESTDUALS

Ait) CONFIDENCE LIUI

MURPR OE ORSERVATIOHS

$? 4$

ALIIRS $\because x$ HOR OBSURMTIOHS

? 30,34

.2 .74

$? 30.47$

3
$? 53.52$

$? 27,36$
n?: $\quad 1.18$
I:ie) VA? $X$
STR ERTOR
CORRTLAMION
DETFRIITHATON$$
\text { H:A.V }
$$
35.2500
42.2500
11.3584
771217
$y$ IUTERCEPT A SLOFE
$-18.5951$
12

STANARO DEVIATEO

8.05588

95 PERCENT CONETDEPCR TIYITS LOHER

$-1.58 .488$

121.238

$-1.96205 \quad 14.55827$

PREIJTELED RESTRIAL

25.5406

43.9055

4.45340
-12.4150

SCUURED

13.3862

154.257

23.1369

$-1.13682$

1. 29236

253.027

HAKE 95 PERCRUT COHEIDENCE THTERYAL PREDICTIONS. YES OR VO

hile:. $X=$

PRPDICTES

LOWRR bThI?

.

$? 43$

TRY AHOZHKR. YES OR 2230

12.6540

UDPER EIMII

51.7832 
TRANSITION ANALYSIS: 6-5 YEAR TREND

DISTRICT 非, KENDAI-UPPER ROCK SPRING (STRATUM \#III)

BIRTH-GRADE 1; GRADES 1-2;

P:?

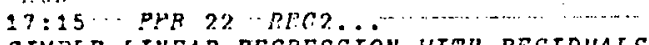

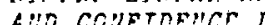

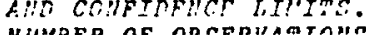

?E

20.33

$2,22.02$

$\stackrel{2}{?} 155,85$

2177.90

$?: 02.73$

6

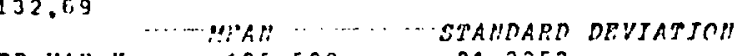

LEP VA. Y $\quad 130.500 \quad 31.3353$

IIID VRS X

CONDEATIOI $\quad .128650$

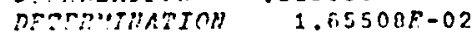

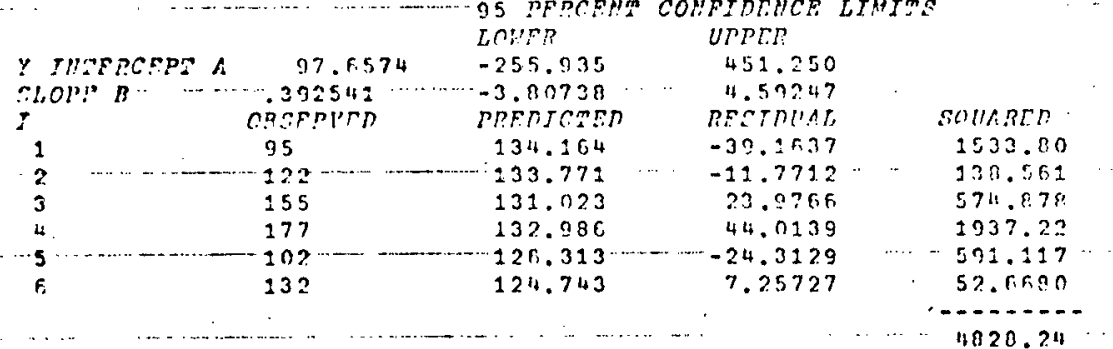

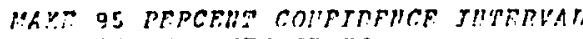

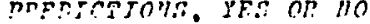$$
\text { ?urs }
$$

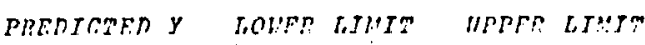

$\because n ? m=$

$? 75$

127.098

73.4769

180.719

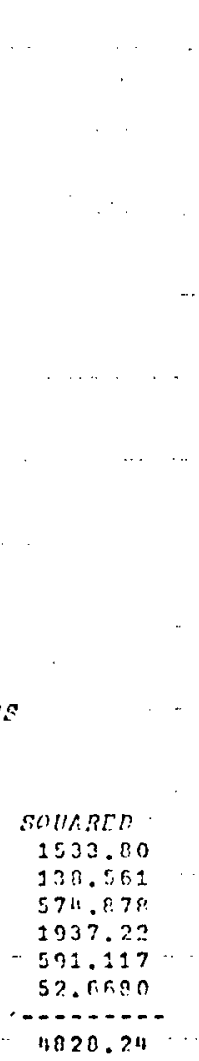$$
780 \text { 114 }
$$

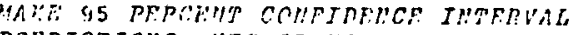
YF:

men:

$? 127$

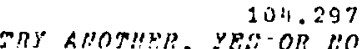

PRTESCRED Y IOHER LI!!r

UIPPP IIIST ?110

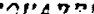
52.8445 230.055 $14,6.334$ 324.207 324.207 812.638 
TRANSITION ANALYSIS: 6-5 YEAR TREND

DISTRICT 非, KENDAL-UPPER ROCK SPRING (STRATUM \#III)

GRADES $2-3 ; 3-4$

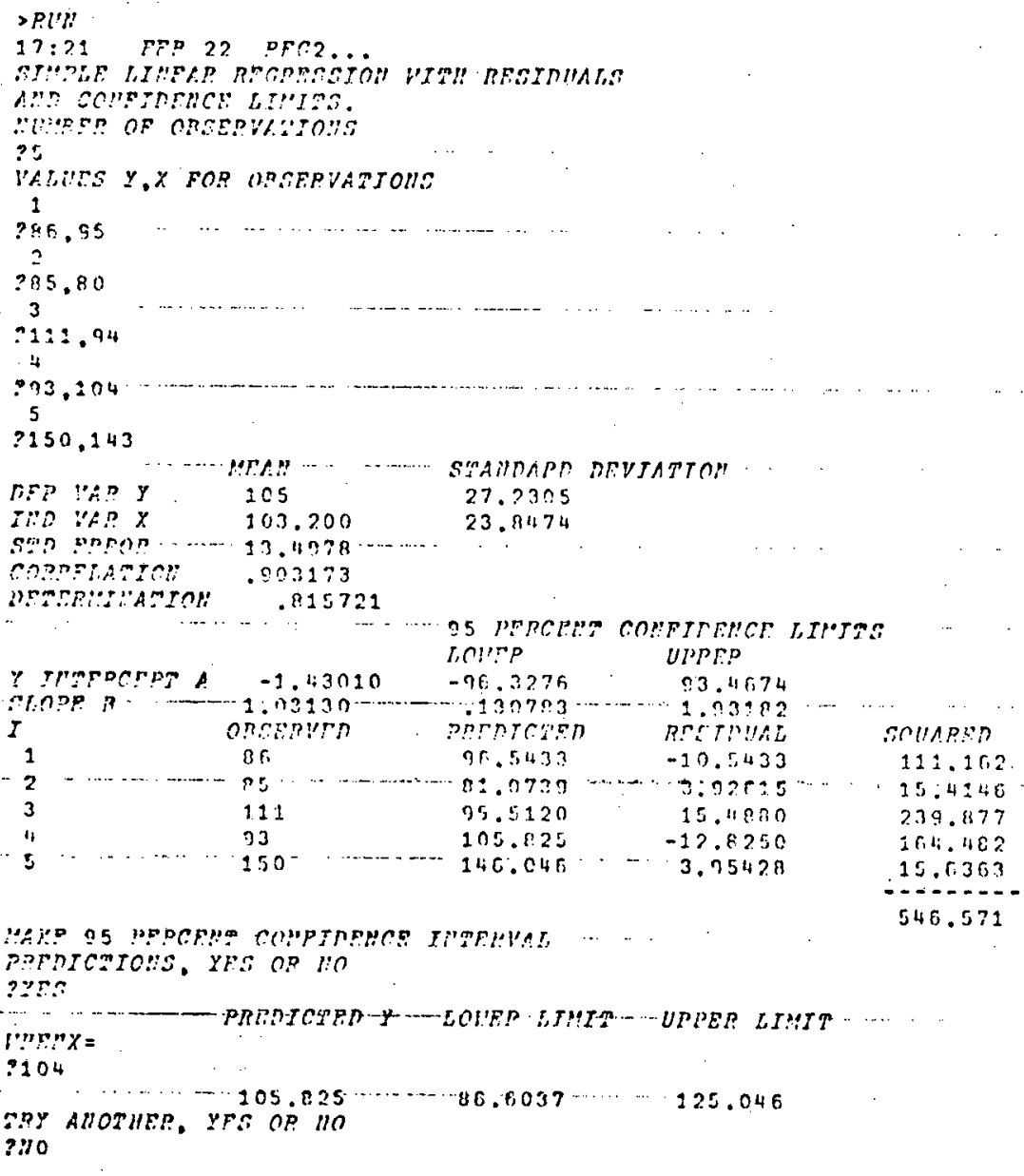

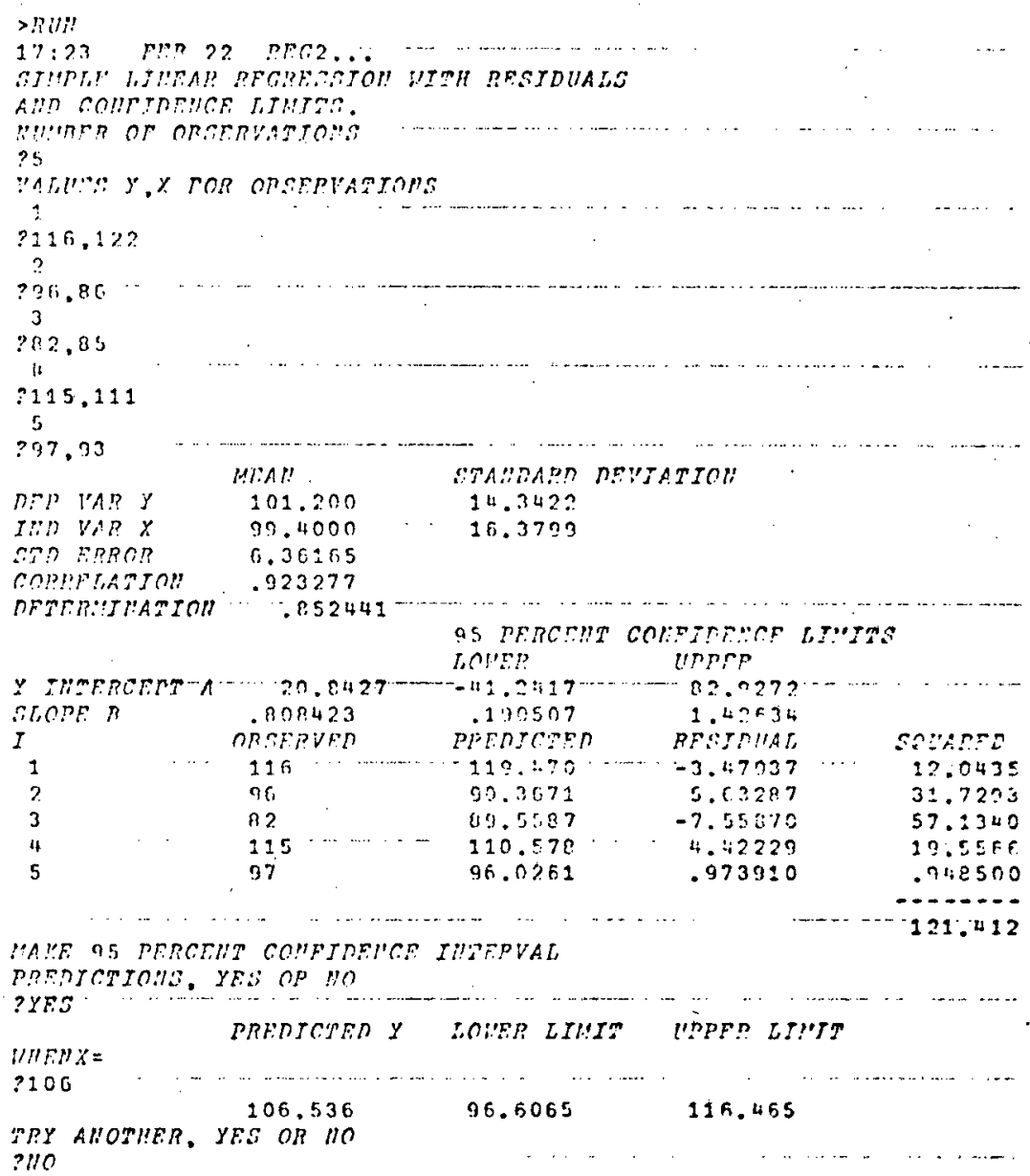


TRANSITION ANALYSIS: 6-5 YEAR TREND

DISTRICT 非, KENDAL-UPPER ROCK SPRING (STRATUM 非III)

GRADES $4-5 ; 5-6$

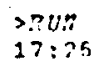

$17: 25 \quad \operatorname{TSR} 23$ RFOS

THEAR PFCRTSGSOH! VIT! RFGIDUALS

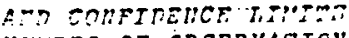

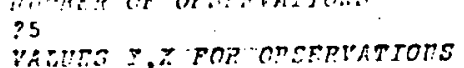

?1014.95

2104.95

$? 258.116$

$\because 21,0.5$

$221,0.5$

2117.82

$\frac{5}{132,115}$

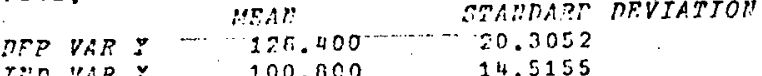

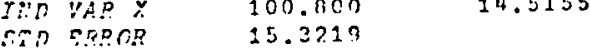

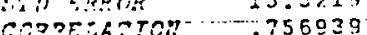

GOREE ARATOH

.

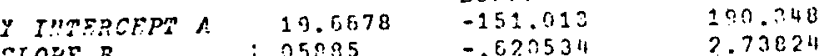

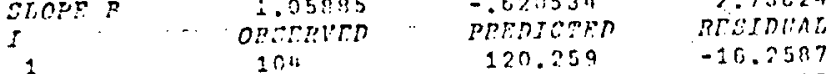

$1 \quad 101 \mathrm{a} \quad 120.259$

$2 \quad \ldots \quad 158 \quad 142.1195$

$\begin{array}{rrr}3 & 122 & 121.318 \\ 4 & 127 & 106.494 \\ 5 & 132 & 141.436\end{array}$

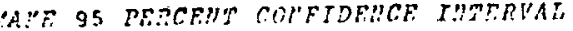

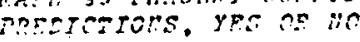

mis:

PRERTEMER Y IOVHR LJ!ITT

15.5055
$-\quad 317513$

$-.317513$

$10.5061 ;$

$-9.113500$

207

132.96 .5

108.803

ITPER LII:I'

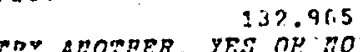

157.127

?110
$>R(/ / !)$

17:29 FLR 22 FllC2.

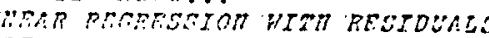

Lit?tis

VALUES Y,X FOP OREERVATJOIS

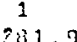

231,93

295.204

35.158

4
$.20,121$

220,121

$? 58.117$

DER UAP $Y$

IIIS YAR

TTR TR?OR

CORPELA THOH

63
118,600

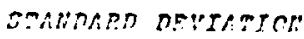

26.4858

10.3539
-.507122

$24,5,42$

FYRIIIUASTOR

SOIARED
254.344
240.1199
100815
120.385
89.0323
704.201

\begin{tabular}{|c|c|}
\hline$\because$ IHTRRCHPT A & 127.6141 \\
\hline$\stackrel{\because}{I}$ & - 545035 \\
\hline 2 & 81 \\
\hline$\cdots$ & .35 \\
\hline 3 & 55 \\
\hline$":$ & 26 \\
\hline
\end{tabular}

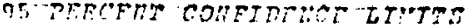

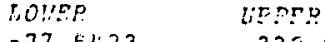

$-77.01: 23 \quad 33 \%$ 2325

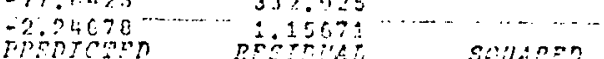

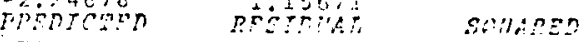

$7.0529 \quad \because .9 \% 43 \quad 16.3750$

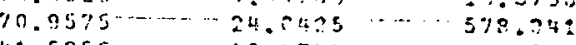

4.5250 13.4744 IR:.550

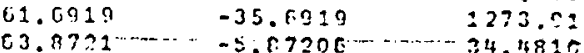

2094.37

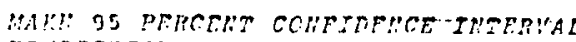

2784.37

polinje'sons, YTS OP 110

$m+m y=$

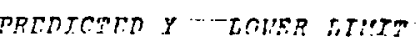

URPTR LJ:YTT

$? 133$

TPY APOTHIR, YES OR IIO

$-20.3457$

99.9563 
TRANSITION ANALYSIS: 6-5 YEAR TREND

DISTRICT 非3, KENDAL-UPPER ROCK SPRING (STRATUM 非III)

GRADES $6-7 ; 7-8$

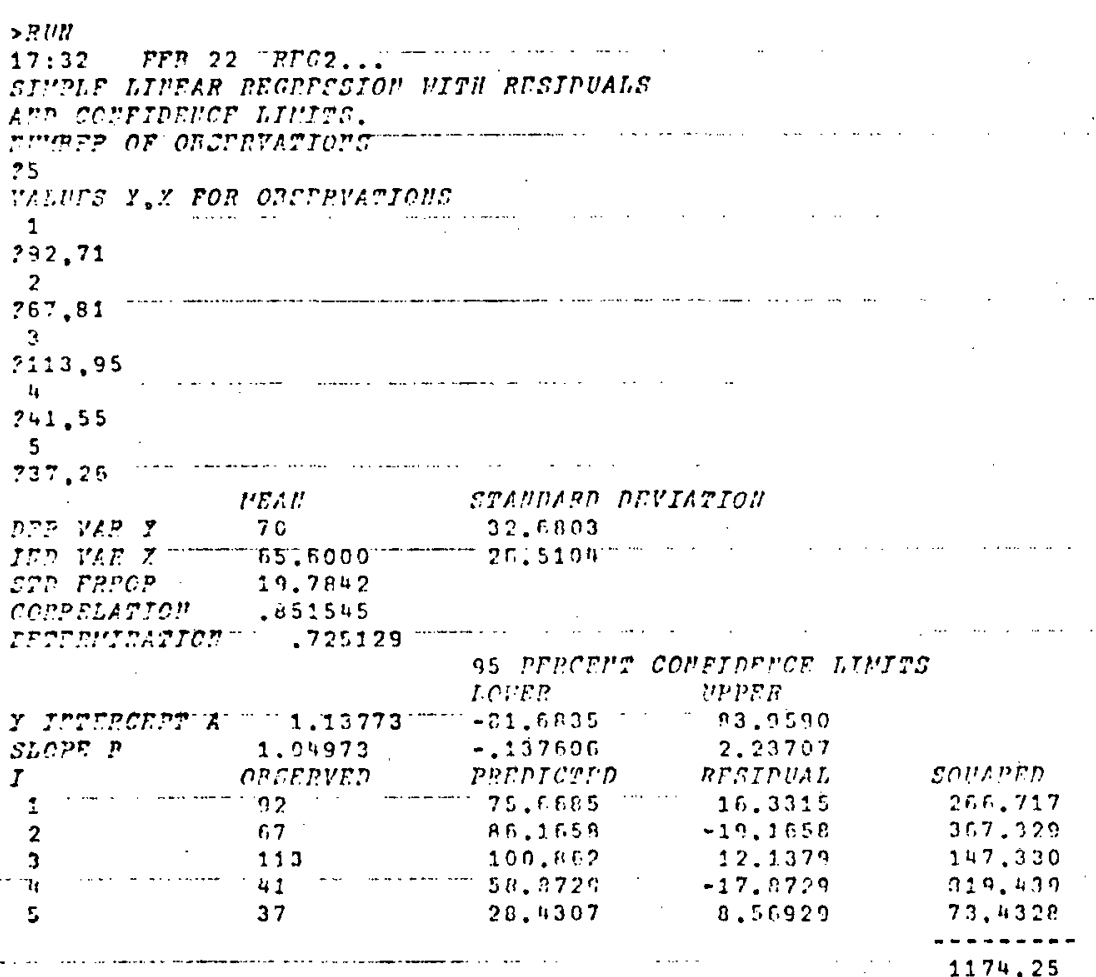

WPY 95 PFPCEMT COUPJDEHCE THMRRVIL

PRPRTCirolid. Yes on 110

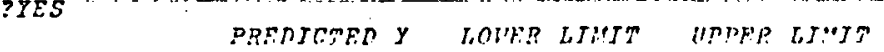

H: $: 1 Y=$

\section{(1.6)}

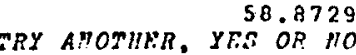$$
>R i: \cdots
$$

$17: 34$ FIP 22 REC?

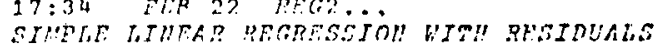

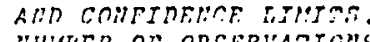

32

35

$\frac{1}{275,72}$

$?$
$? 40.92$
3
$? 83.67$

?83.6.7

$? 28,113$

$? 20.41$

DIP $V A R \cdot$

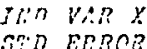

COR ERTRE

CORREIATIOH

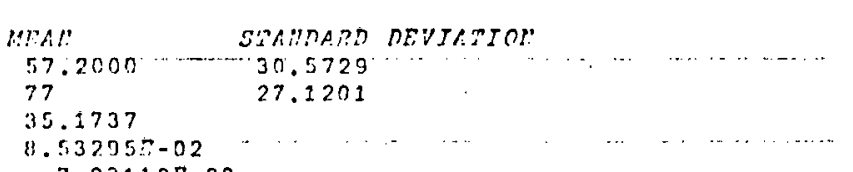

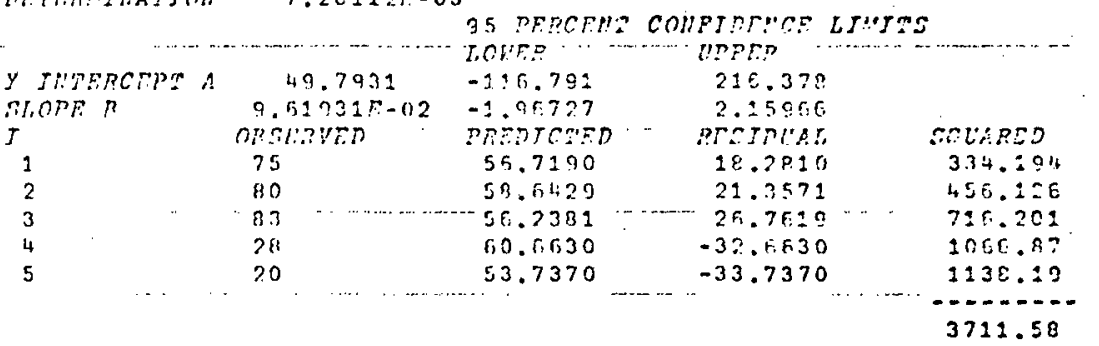

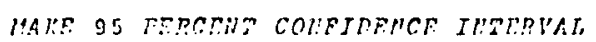

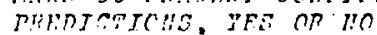

?YTA

PRTRECTET y LOWTR LIMTT UPREP LITIST

$\operatorname{lntrm} x=$

550

TPY AROTILR, YSS OR 170

$-6.86047$

117.798

$? 110$ 
TRANSITION ANALYSIS: 6-5 YEAR TREND

DISTRICT 非3, KENDAL-UPPER ROCK SPRING (STRATUM 非III) GRADES $8-9$

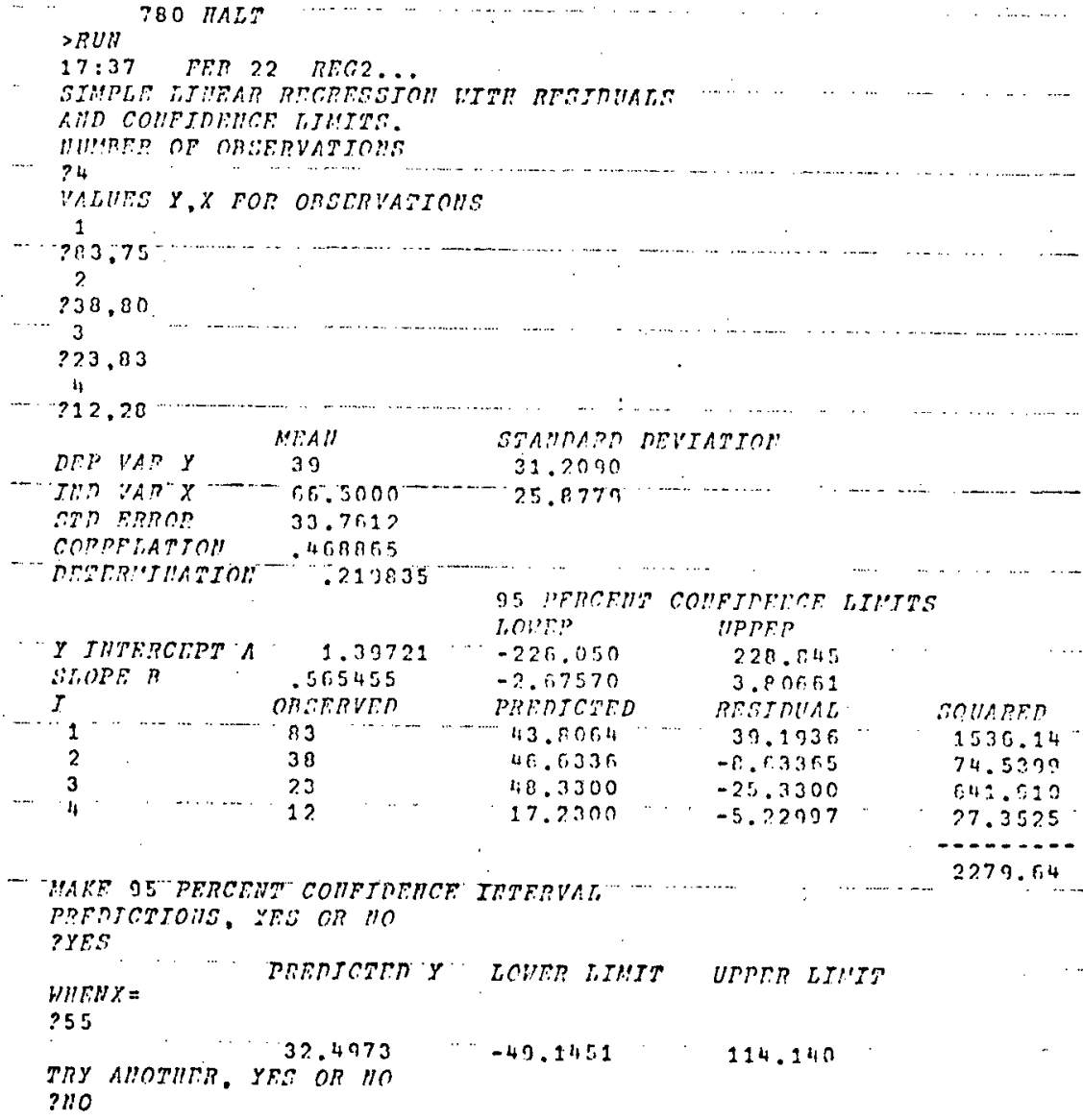


TRANSITION ANALYSIS: 6-5 YEAR TREND

DISTRICT 非6, JERICHO (STRATUM 非IV)

BIRTH-GRADE 1; GRADES 1-2

$>?, \ldots$

$07: 28$ FF3 23 36C2

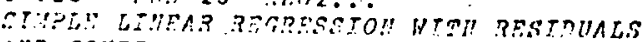

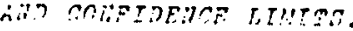

$?$

TIJTS Y, $x$ FOR ORSERVATIOUS

$\stackrel{1}{?} \div 03.110$

2125,10

$? 3.105$

$?: 450.107$

5

$? 110, ?:$

$: 102.77$

\begin{tabular}{|c|c|}
\hline & mith? \\
\hline 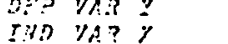 & 114.667 \\
\hline 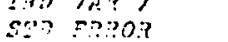 & 33.5557 \\
\hline rronthas? & $\begin{array}{l}21.035: \\
.3701600\end{array}$ \\
\hline 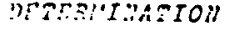 & .137196 \\
\hline$\cdot$ & \\
\hline 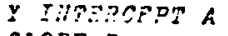 & 57.2270 \\
\hline 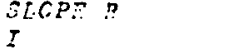 & .573318 \\
\hline${ }^{2}$ & OSA PVER \\
\hline$\frac{3}{2}$ & $\begin{array}{l}108 \\
125\end{array}$ \\
\hline 3 & 93 \\
\hline$:$ & $15 n$ \\
\hline 5 & $2: 0$ \\
\hline 6 & 102 \\
\hline
\end{tabular}

STAMAMPD REVTATEOA

20.2550

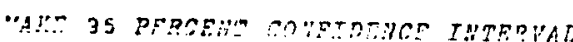
botrchione. yes o. no

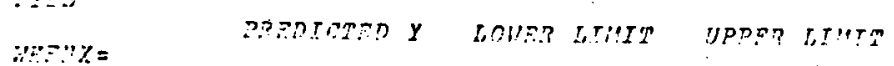

IPPET LIITI

83.4905

138.159
$>$ ?⿻:木

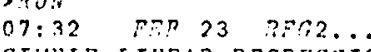

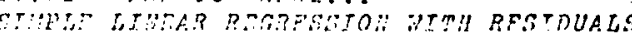

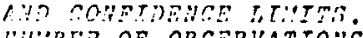

$? 5$

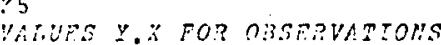

2

$? 34.108$

$? 23.125$

$? 96.93$

$? 113.150$

$? 217.110$

nes wis y

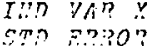

ASAH

100.500

117.200

DTAMTR DEVTATION

14.1174
21.5569

copo:10m

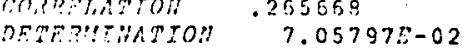

$$
\text { - }
$$

$y$ timtaresan a

80.2032

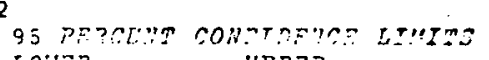

SIOPE

Sก)

153.314

30.5385

357.5116

357.5146
.107642

.157243

$\therefore 1700.00$

I.

2

3

5

"ANF 35 PERCEMT COURTRFUCF TYARPVAL

PBEDTCEOAR, YES OP NO

?1:3

0.173998

34

8.3

96

113
119

$-57.5505$

$-9885890$

39.9934

101.957

315.3896

105.307

עen:?

217.374

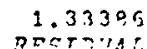

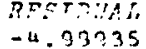

-4.33735
-18.9571

$-18.9571$

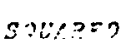

$6.67335 \quad-151793$

17.6527

44.9203

311.617

740.334

,ir:T:Y=

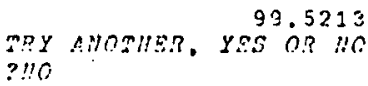

76.0298

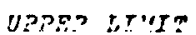


TRANSITION ANALYSIS: 6-5 YEAR TREND

DISTRICT 非, JERICHO (STRATUM 非IV)

GRADES $2-3 ; 3-4$

760 HALT

$07: 34 \quad Z F R \quad 23 \quad R F C 2 \ldots$

THPLE LIUEAR BFCBRSEIOH HITH RFSTDUALS

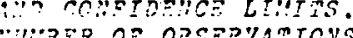

$? 5$

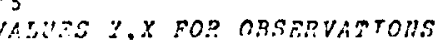

$? 74.53$

5

992.34

$? 31.83$

$? 87.36$

$? 87.36$

$? 110.113$

LEA." STAMARE DEVTATTOM

DEP VAR Y $95.8000,15.6423$

s.7 $3300 \% \quad 6.97130$

0.8430

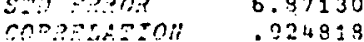

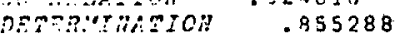

\begin{tabular}{|c|c|}
\hline In:s?⿻上丨 & -42.946 \\
\hline SLOPE 3 & 1.33347 \\
\hline$I$ & OกתBRV=? \\
\hline 1 & 74 \\
\hline 2 & 32 \\
\hline 3 & $\begin{array}{l}72 \\
37\end{array}$ \\
\hline 5 & $1: 0$ \\
\hline
\end{tabular}

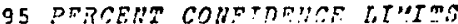

IOWE?
$-137.376 \quad$ IPPF?

3257390

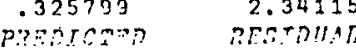

8.0063

34.3937

69.7315
87.0667

$: 09.738$

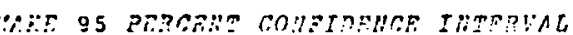
parentegrons, yes o. no

?XSS

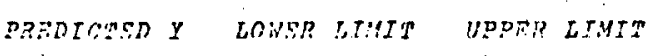

MUE. $X=$

2100

92.4005
YOY AUOF!ER. YFS OP 110

31.7453

103.255
-3.06627 SOUAPFD

7.50725

$-8.067505-02$

.254232

82.1373
57.7539
1.60304
$4.448225-03$
$5.382815-02$
(..........

141.044

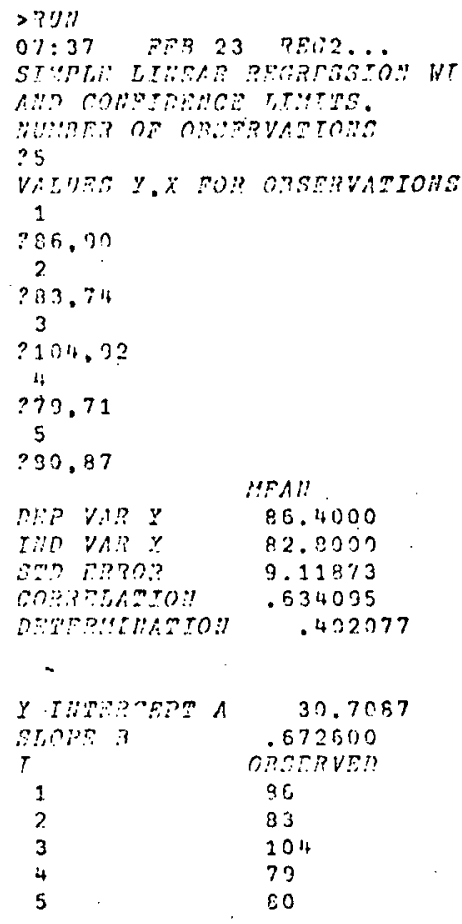

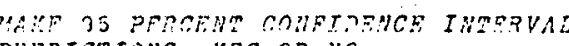

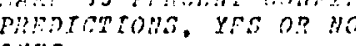

?Y:S

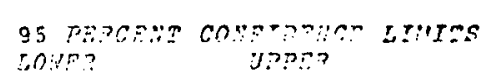

$-34.7239$

- 2034231

31.2427

92.5879

89.2249

Jise?

155.147

2.17343
$0.9+2010$

$-5.21: 272$

$2.5: 333$

11.4121

.536577

$-3.22432$

27.4851

E. 34475

132.235

$.2000=3$

85.0331

249.454

min: $x=$

PRADTCRED Y

LOHE? LTYTT

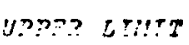

$? 32$

TRY ANOFIFR, YRS OS?

73.5935

111.576 
TRANSITION ANALYSIS: 6-5 YEAR TREND

DISTRICT \#6, JERICHO (STRATUM 非IV)

GRADES 4-5; 5-6

7 a日 $P$ A ILT

07:40 587 23 7FG2

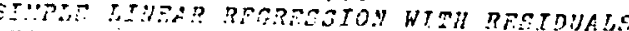

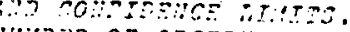

35

Vheyes y.x ron onernyarons

$? 22.90$

33,25

$? 39,83$

239.134

$\stackrel{5}{292,73}$

$.92,73$

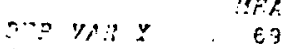

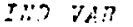

89.5000

SIMAINASD DFVIATION

St? Popor

coppgingron

26.5656

23.4201

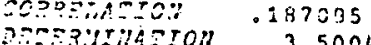

$3.500452-02$

S T:OERCEPA A$$
30.5570
$$

DOH?
-342.179

.420351

-342.179
-3.70350

ONSERVE

8 ?

33
59

59
79
32

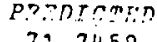

$71.745 ?$

7.7 .4554
60.02033

75.1793

64.4521

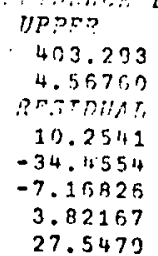

Solvapin

105.116

1197.19
51.3948

11.0051

$75 \dot{8} .839$

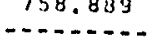

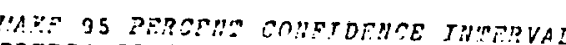

PPFOTEETOHS, YES O?

peEnterso y

LOWR? L.T:HT

UPPER ET:'TT

$\operatorname{21:2:2x}$

30.1212

110.736

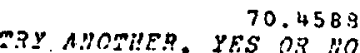

30.1212
2117.20
730 RALI

$07: 43$ PR? 23 REC2..

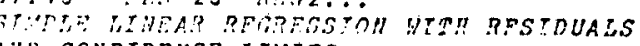

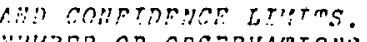

5

WATUES $7, x$ FOR ORSERVATIONS

: $90, f 7$

2
$? 64.82$

3

230,33

$? 30,59$

5
$\therefore 8.79$

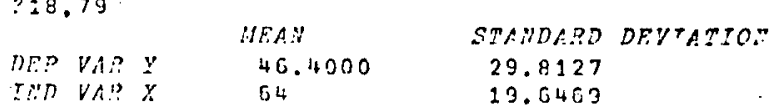

STI FIR?OR

CORpETATIOAI

33.2295

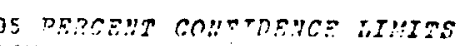
¿ou? ? "mpl"

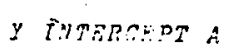

STOPE?

21.0321

.396373

$-157.56$

$13 ? .625$
3.03539

?

1

30
64

$-2.23455$

DRPRTER?

47.5819

53.5347
34.1124

34.1124

3.4 .4181
52.3456

3.03732

39:T D\%:

10.4553

$-214.1491$

$-34.3456$

1730.58
109.532

10. .2121

207.033

18

1179.52

3312.62

MAKR 95 FFRCEIIT COUFTDEUCE TUARPVAL

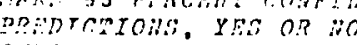

$\operatorname{Br}: 1, x=$

ponotogen $y$

LOHER LI:UTT

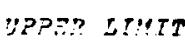

.70

TPY AllOTHES, YES OP 19

$-1.18900$

98.7455 
TRANSITION ANALYSIS: 6-5 YEAR TREND

DISTRICT 非, JERICHO (STRATUM 非IV) GRADES $6-7 ; 7-8$

780 HAL

$2 ? 13: 1$

$27: 46 \quad r=323 \quad B E r 2$

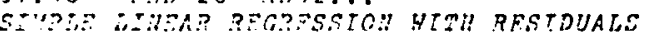

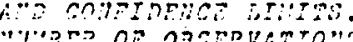

$? 5$

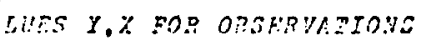

257,54

5

$2 \times 3.30$

$? 75.5$

$0,35,30$

5

280,30

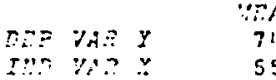

E.s? sosos

6r?

$74.200 \%$

55.0000

10.3096

STABMARD DEVTAMTOA

15.2545
25.6572

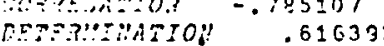

\begin{tabular}{|c|c|}
\hline $\begin{array}{l}Y \text { FOEROEPA A } \\
\text { SLOBS B }\end{array}$ & 100.143 \\
\hline 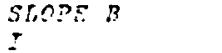 & -.465506 \\
\hline$z$ & OBSPRVRD \\
\hline 1 & 57 \\
\hline 2 & 53 \\
\hline 3 & 75 \\
\hline 4 & 96 \\
\hline 5 & 80 \\
\hline
\end{tabular}

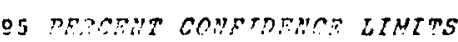

ion?

$59.4050 \quad 140.322$ $-1.14285 \quad .203539$

PREAECTED RESTRUAT.

70.2305

58.1488
70.2905

86.1451

36.1451

13.2805
4.85124

4.85124

9.85439

$-6.14511$

SCllaren

176.372

2.3 .5310

2. $2 \div 31$

37.1188

.37 .7524

357.251

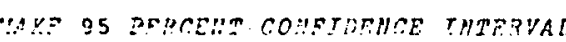

wentCrions, YES OR NI

$3 x \sin$

$\because: 2 ! x=$

sparamen

SOLPD RTIST

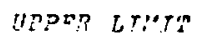

343

MPY A.IOMETE, Y:S OP? 270

61.1260

93.4332
$>$ ?.?!:

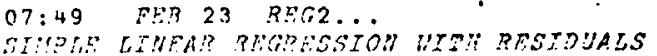

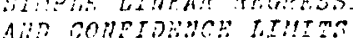

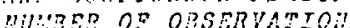

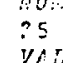

VIIJES Y,X POR ORSERVATIOHS

2

$3: 9.47$

$? 260.57$

$? 37.63$

4.63

257.75

$? 52,96$

DEP VAP

STD Fis?

conpesenton

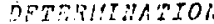

YISA.V

53
67.6000

EAAITARD REVTATTOH

0.24534

5.43139

$9.04147 \%-02$

y rUTHCEPTA

$8.174017-03$

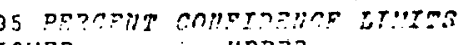
EOI:?

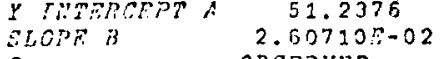
24.4933

1

2
3
4

5

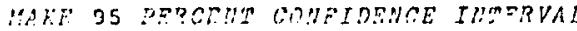

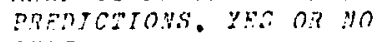

?YSS

OREE?VE?

49
60

47

57

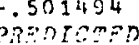

52.4529

52.7230
$52.300:$

52.510 .01

53.12 .29
53.7404

07.3919
.553536
0.517048
-3.45794
7.27635
-5.80707
3.00707
-1.74042

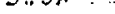

i. 1.2913

52.7453

$34.27=3$

24.439.

3.02305

117.035

Hi:m =

$? 77$

PRENICTED Y LOUER LIHIT UPPFO LIUIT

TRY AHOTIER, YES $0 . ? \cdot 240$

43.0670

63.4231

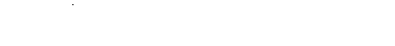




\section{TRANSITION ANALYSIS: 6-5 YEAR TREND DISTRICT 非, JERICHO (STRATUM 非IV) GRADES 8-9}

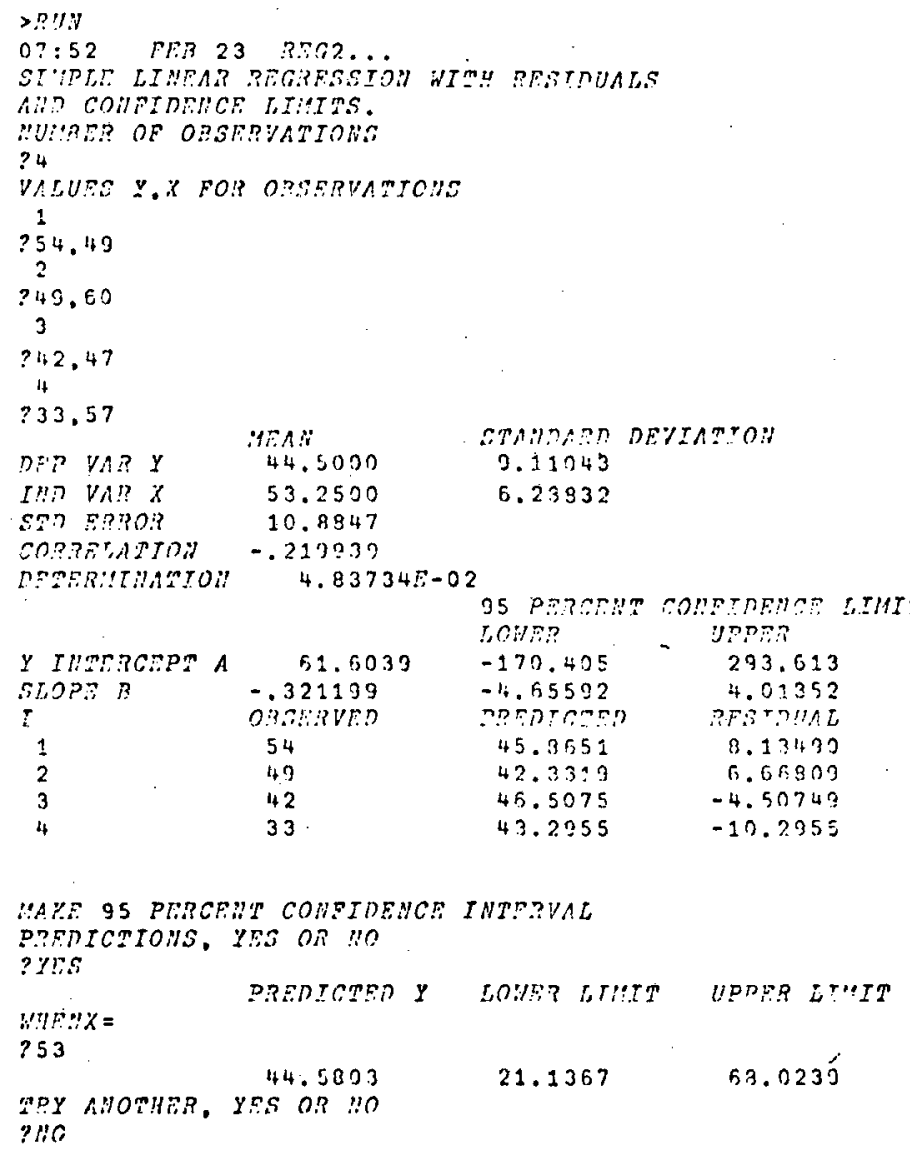

2

UARE 95 PLRCFUT COMSIDENCE TNTERVAL P.PEICTIONS. ZES ON NO

\section{?TIS}

Ut: :PX =

PREDECTEA Y LOHET LIIITT UPRPR ET'IT

$? 53$

44.5803

APY AHOMHER, YES OR "NO

21.1367

5月. 02305

?HC 


\section{庹}

\section{SOIPPED}

6C. 1767

44.4535

20.3175

105.327

-........

236.955 
TRANSITION ANALYSIS: 6-5 YEAR TREND

DISTRICT 非, LUCEA (MIXED STRATUM)

BIR'TH-GRADE 1; GRADES 1-2

21:32 FEP 22 REG2.

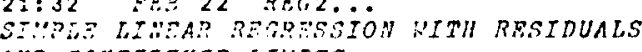

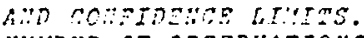

GELUNA Y,X FOR OPSERVATYONS

1
$? 254.535$
2
$? 221.522$
3
$? 232.509$
4
$? 243.481$
5
$? 249.517$
5
$? 295.510$

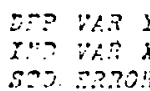

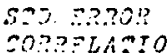

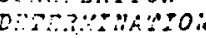

Usal:

STAWDART DEVTATHON

$247.167 \quad 22.1126$

513.500
24.6351

18.9021

7. $365195-03$

35 PRACRUT CONFIDRHCE LIUTTS

Y $\operatorname{TATREEPTA}$
SLOPE $B$

297.1420

LOWE.?

$9.786535-02$

2

2
3
4
5

5

254
221
231

231
243

249

285

$-529.958$

JPPE?

1124.80

1.53 247

eptom

245.003

245.650

247.007

250.347

246.824

247.509

c. 33744

5,498

7.34729

2,17586

37.4903

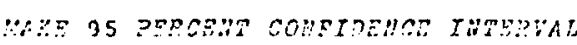

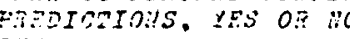

PYES

PBARICTED $Y$

LONER LIUIT

UPPER LI:ITS

$\frac{12 m a x}{2536}$

TRY AIOOMER, YES OR 110

199.223

$290.70 \mathrm{E}$
RU:

$21: 35$ FSR 22 BRC2,

CT:PRE LIBRAR RUCRESSIOI WITII RESIDUALS

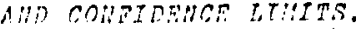

MU:RER OF OASERVATEOW

YALUES $X X$ TOR OBSERVARIOHS

$? 200.254$

2196,221

$? 213,231$

$? 232,243$

$? 204,249$

nep

I.:? WiR $x$

GT? RM? Q?

cospotaitson$$
\text { MEA: }
$$

STARTADD DEVATEOH

$209 \quad .14 .3173$

33.4833

10.3673

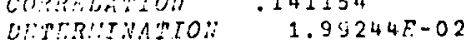

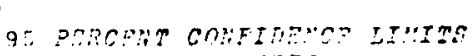

Lo:?:?

y IITSRCPPT A

173.085

0
0
0.90908000

S6)

79.9773

607.610

275.734

53.9226

4.73437

1405.56

2427.56

200

$-290.238$

$-1.78 \pm 41$

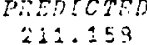

211.959
265.212

207.711

$\begin{array}{llr}2132 & 223.510 & 22.4904 \\ 204 & 210.409 & -6.40897\end{array}$

we?

$636.41:$

2.0812
anding

$-1: .159 .+$

$-10.2220$

22.4904

$\begin{array}{rrr}23 \% & 223.510 & 22.4904 \\ 204 & 210.409 & -6.40897\end{array}$

Scy?

120.510
004.225

27,0749

525.017

5.017

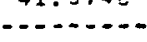

WHE gS PHCEIT COMFTHFUE IMTERVAL

303.652

pornentons, Ias on :O

?Y:

PREDICTEO $y$

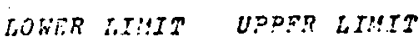

, ? ? $: 3 x$

$? 245$

TRY AHOTHER, YES OR NO

184.290

2.35 .329 
TRANSITION ANALYSIS: 6-5 YEAR TREND

DISTRICT 非1, LUCEA (MIXED STRATUM)

GRADES $2-3 ; 3-4$

$>$ ․ำ

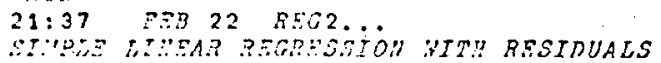

$\therefore$ congto-uas tritmo

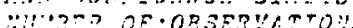

YAUARS $x, x$ POR OESERVATOOHS

$\frac{1}{2} 278,250$

2

$226 a, 200$

$3^{3} 28,106$

it:

$? 242,213$

2269.232

Dip

-

oppo-intarn

\section{"EA:"}

259

STAWDARD DFVIATIOA

27.1172
25.2326

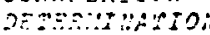

5. $85725.5-02$

$3.43073 ?-03$

35 PERCPHT CONEIDENCE LIHITS

LOIISP UPPAR

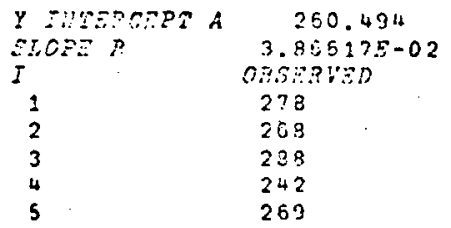

$7.3025 \mathrm{E} \quad 528.230$

$-1.17180 \quad 2.24921$

PREDTCTER RESIDUAL

270.508
$259.227 \quad-740213$
290.078

$203.072 \quad 19.3279$

$\begin{array}{ll}208.729 & -25.7294 \\ 269.464 & -.463941\end{array}$

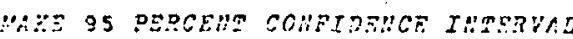
PPFORGTIOHS, YES O? HO

YYES

:ytr:ax $=$

PERDICTED Y LOHER LIMIT UTPER LIIIT

2210

TPY ANOMYSR, YSS OR 268.513

238.037

299.190

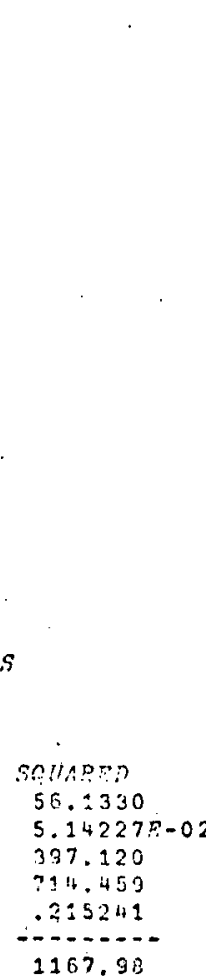

\begin{tabular}{l} 
Selinp? \\
56.1330 \\
$5.142279-02$ \\
397.120 \\
714.459 \\
.215241 \\
\hdashline 1167.98
\end{tabular}

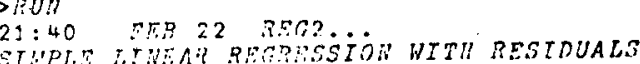

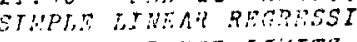

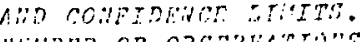

?

2194,257

? 205,278

3

$? 134,263$

$? 180.288$

3

$? 205.242$

nir VAR $y$

MEA:

STANDAPD DEVIATTO:

tho $\forall A !:$ :

187.558

15.4020

17.5275

127.597

copsentivon

.1691172

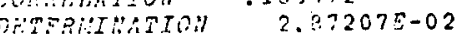

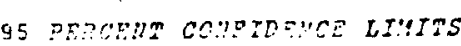

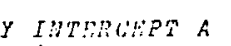

123.012
$2.213635-02$
$035 \% \operatorname{lon}$
194

$0 \%$

127.535 0.214032

138.723

138.723
103.183

105

194

130.283

183.967

183.019

188.330

यर八?

233.502

$\sin 25330$

5.27598

$-24.2891$

5. $0: 292$

so 8465

87.8455

25,3233

$7,4 \leq \leqslant 08$

775 .

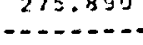

321.635

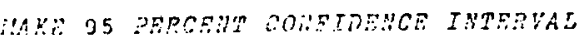

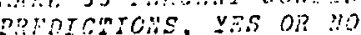

?XES

LOWER LIUTH

نD??: Lx::T?

$\operatorname{mrg} r=$

2269

TRY ANOTHER, YAS OP IIO

160.274

237.704 
TRANSITION ANALYSIS: 6-5 YEAR TREND

DISTRICT $\# 1$, LUCEA (MIXED STRATUM)

GRADES $4-5 ; 5-6$

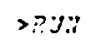

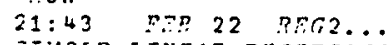

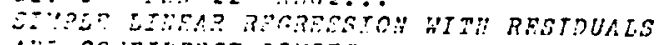

A.

$? 5$

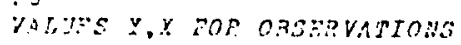

: 232,265

$2: 33 .: 94$

$?: 34.105$

205.254

$\therefore E 2.130$

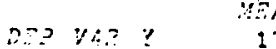

a.d

?? $=? 0$ ?

n?

$1 ?: .200$

170.500

14.3624
.373027

.132597

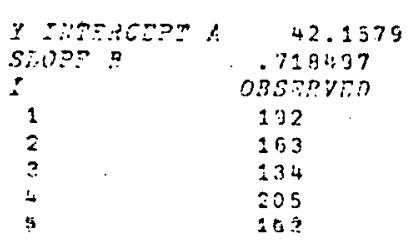

STAMDARD DEVIATCH

27.8273
14.5017

14.5017

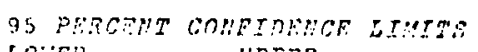
LOFE" . UPFEs

$-547.325 \quad 6.22 .240$

$-2.55552 \quad 3.93550$

DPFDICERA RESTRUT

16.5. 710

191.540

100.710

101.5165
171.497

31.2901

$-13.5454$

-28.7993
2.3 .4536

$-3.48740$

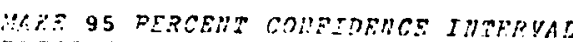
pongremans. HES OP nO $x=2$
in:?x $x=$
PRSDICTSD $Y$
LOHER LIMIT
UPPER LIHT
2189
TSY A.:ORHRR, YES OP 170
125.460
230.447

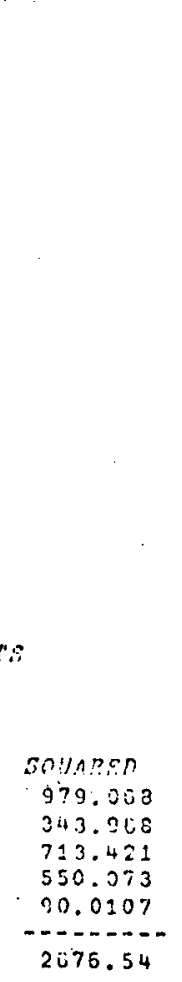

134.247

MRY AIOTUER, IFS OA

.35 .8305

232.664 
TRANSITION ANALYSIS: 6-5 YEAR TREND

DISTRICT \#1, LUCEA (MIXED STRATUM)

GRADES $6-7 ; 7-8$

3.0 .04

$21: 43 \quad \therefore 22$ REC2.

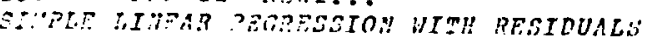

a

?

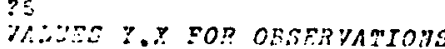

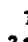

$\therefore: 20,202$

?

$?: 2 \geq 0.215$

2115,110

$24,5,54$

$? 45.5$

$? 1 \geqslant 0,115$.

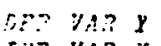

-

cop?:17:0

:2A:

113.800

143.400

33913

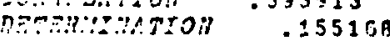

STAIMAPD DEVIATTON

54.3847

62.3562

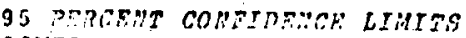

SOlis?

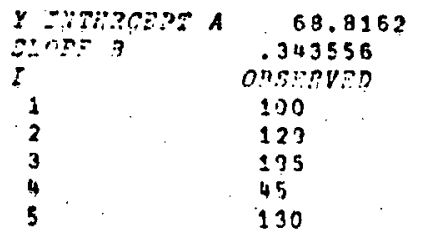

$-104.562$ -1.12917 .

P.RกT:TED

138.215

11.062

190703

108.325

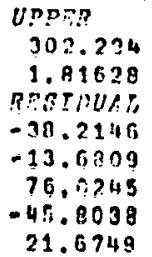

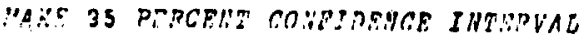

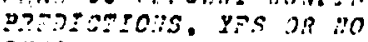

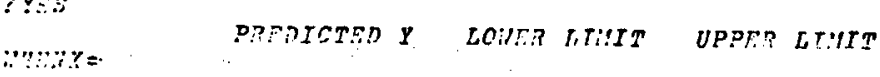

134

30.0207

199.685

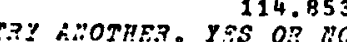

2.110

$>$ PU:

$21: 51 \quad$ FEB 22 pros

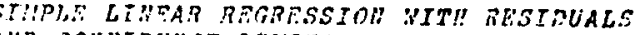
Wh collerbilles LYMTte.

$? 5$

WILUES Z, $x$ FOR OBSESVATIONS

$? 115.123$

112,100

? 116,129

?"152,195

$? 100.43$

De? VA.S

He:

120.200
118.400

9.01359

STAMATS DFVEATECH

18. 9339 .

类

renipinton

.903339

FTERIIIAHION $\quad .896022$

Sก!กา?

1450.36

197.166

5773.73

2.297.

$46 ? .736$

9925.64 y TUTRRCEPT A

814.2515

.303619

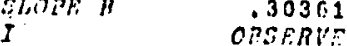

1 i15

i15

112
110
152

1150
106

$20 \%$
$20 \%$

50.3714

3.976345 .02 PREDTCTES

121.537

114.513

123.429

97.9144 .

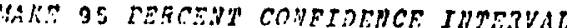
PREDICTIOAS. YES OR iO

?YES

Hite: $=$

PREDICTEN Y LOHER LIEIT UPPER LTMTT

?115

TRY AHOTHER, YSS OP. MO

106.309

132.026

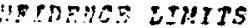

ע.

$1: 3.135$

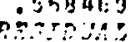

$-5.59655$

$-2.53249$

7.49230
9. 54279

8.08563

S?:Bi?

$47.5: 58$

8.93932

72.9733

65.3774

-........

243.734

?iio 
TRANSITION ANALYSIS: 6-5 YEAR TREND DISTRICT 非1, LUCEA (MIXED STRATUM)

GRADES 8-9

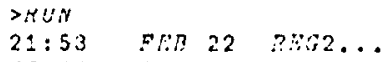

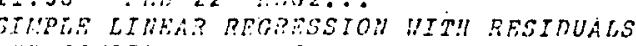

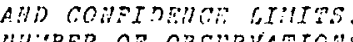

$? 4$

WhituRs Y X FOR OPSERVATIOHS

1

$? 96,: 15$

2

$? 58,116$

$? 58,116$

$? 82,152$

DEP VAR

$\because E A: ?$

81.2500

ETATLAD DEVIATTOL

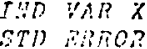

1.23 .750

16.5202

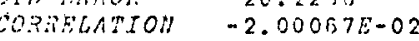

COREELATIOH - $20.00067 E-02$

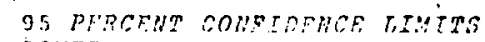

Lown. UIPPE?

$\begin{array}{lc}Y \text { IWMERCEPE A } & 83.4130 \\ \text { SLOPE } & -1.747247-02\end{array}$

$-248.33$ $-2.57512$

81.4029

31.4554

31.3355

80.7552

4.103

2.64015
0.570 .15

14.5971

7.54403

7.54403
-23.2955

2.24377

SOldinges

213.074

56.3214

1.5400

………

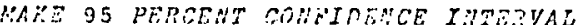

813.422

PREDICATOHS, YRE OR "IO

?.85

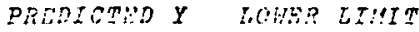

UP.PR LTEIT

Win:

$\$ 119$

81.3330

35.0206

126.649

3110

780 IIALT 
TRANSITION ANAIYSIS: 6-5 YEAR TREND

DISTRICT 非, GREEN ISLAND (MIXED STRATUM)

BIRTH-GRADE : 1 ; GRADES $1-2$

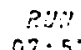

$07: 57 \quad F R \quad 23 \quad 0=02$

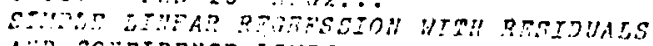

$x \cdots=0$ os

$? \varepsilon$

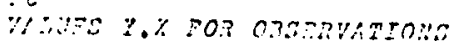

2345.443

$2,30,430$

3

$? 3: 46.40$

2425,1424

$\begin{aligned} & 5 \\ = & 459,385\end{aligned}$

6
2355.335

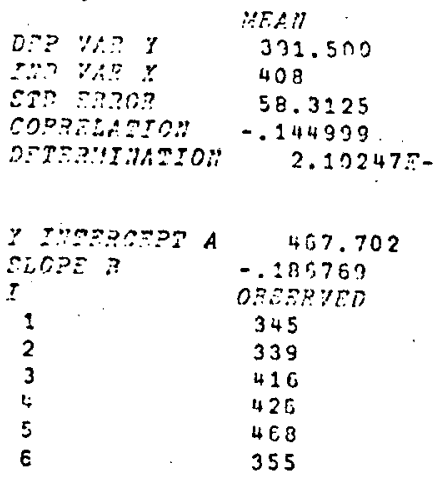

SMAMPABR DEVIATTO:

52.7134

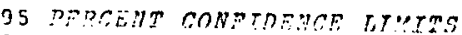
LOUF? UPOR?

-257.01:8

$\begin{array}{rl}-1.3557 \% & 1.58218\end{array}$

pirntomat

384.963

335.710

387.331

3.37 .373

395.005

404.947

RESTRUAL

$-32.3631$

-46.7102
28.5089

28.5089
35.3200

72.3011

$-49.9473$

YAY 95 PRRCEH: CONEIDENCE THTRRVAL

POPSOTCYONS, YES OR nO

PYFS

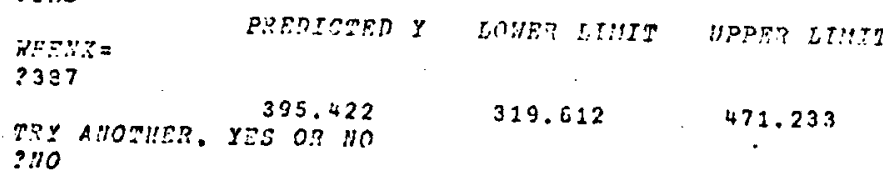

$>: ? y ! n$

03:00 FE3 23 र्वC2.

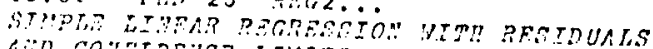

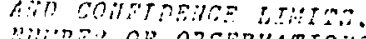

?is

Viuss y.: For oringuntons

$? 396.345$

2297,330

3

$? 354.416$

$? 300,426$

5

$? 431,468$

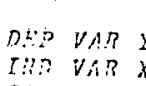

IFA:

371.600

398.807

44.2372

STAINARD DEVTATSO.

50.1727

consocutin .645

Destritris?on $\quad .416955$

$$
\begin{aligned}
& \text { YTERACPEA A } 132.557
\end{aligned}
$$

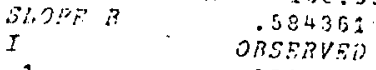

$$
\begin{aligned}
& 1 \\
& \begin{array}{r}
336 \\
297
\end{array}
\end{aligned}
$$$$
1537.05
$$$$
\begin{aligned}
& 2181.34 \\
& 310.1470
\end{aligned}
$$$$
1269.83
$$$$
5 ? 40.47
$$$$
21.34 .714
$$

13001.4

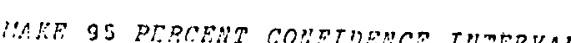
PREDTCTYAC, YTS OR 110

$$
\text { ?YSS }
$$

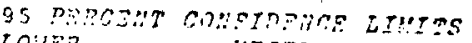
LO:1\%?

$-371.012$

$-.635124$

PREDTCRER

340.161

321.653

32.1 .652
387.435

412.038

य?:?

048.725

a. 35325

postntit

55.8395
-37

$-37.6552$

$-27.55: 0$

$-7,40+40 ?$

$\operatorname{senap=?}$

$3: 17.55$

3572,34
25,4573

36.2533

353.555

5870.82

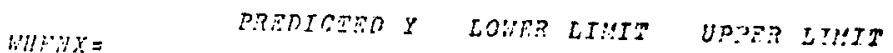

.205

TRY AIOTHER, YES 369.373

306.244

432.515

?"WO 
TRANSITION ANALYSIS: 6-5 YEAR TREND

DISTRICT \#2, GREEN ISLAND (MIXED STRATUM)

GRADES $2-3 ; 3-4$

$>$ P.::

$02: 33 \quad F 5=23, z=62$

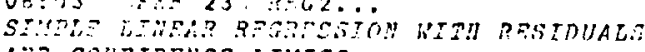

,....

35

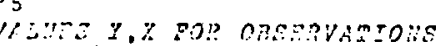

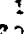

2291.320

$? 2337.390$

$? 273.23 ?$

4353.354

$? 320.380$

\begin{tabular}{|c|c|}
\hline$\forall:: \because: \because$ & 320 \\
\hline$\because \because \because 38$ & 349.400 \\
\hline $28: 02$ & 28.5857 \\
\hline ?ES:EIOR & .319115 \\
\hline 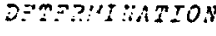 & .670950 \\
\hline IUTERGPPT A & 28.0863 \\
\hline ops $B$ & .861230 \\
\hline I & ORserven \\
\hline 1 & 231 \\
\hline & $\begin{array}{r}337 \\
270\end{array}$ \\
\hline & 359 \\
\hline & 300 \\
\hline
\end{tabular}

SIMTRRO DEVTARTON

43.1557

19.0403

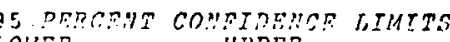

-361.134 UFR?

$\begin{array}{rl}-36153402 & 417.357\end{array}$

DPFDTCMOR RFing

$303.630 \quad-12.6738$

$369.123 \quad-32.1333$

$332.962 \quad 25.0383$

355.354

UHE 95 PERCEW COHEITENCE IUMFRYAL SPEDECETOHS. YES OR WO

?YES

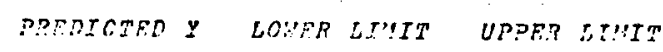

$\because 369$
$? 36 x=$

SYY A.NOTIER, Y:SS OR NO

299.768

391.933
$>$ PU:

$08: 05 \quad 2 R R \quad 23 \quad \operatorname{ReC2} 2$

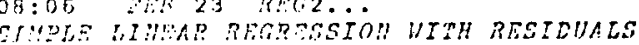

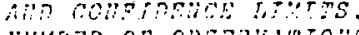

$? 5$

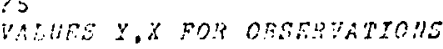

1

$? 212,242$

2255.291

$? 29+3.37$

$? 231, .275$

5
2312,358

rep yil?

Fio vis?

Smb rizpo:

IFAH

259.400

301.400

6.37097

STEINAR: DEVIATION

39.6333

329

Conpetslaton .990263

000

$y$ THerreris A

4.42201

TOYARER

150.778

1032.55
23.7321

525.

607.443

2451,42

i.

i

3
14
5

5

.845579

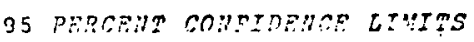

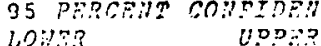

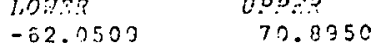

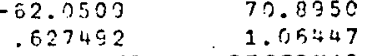

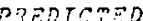

203.149

250.502

240.450

$\begin{array}{rrr}234 & 240.450 & -6.45 .709 \\ 312 & 307.232 & 4.71760\end{array}$

xpot?

5.

5.51504

0.5100

$\begin{array}{rrr}234 & 240.450 & -6.45 .709 \\ 312 & 307.232 & 4.71760\end{array}$

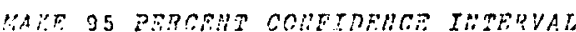

predrctrous, yes or to

$\because \cos$

ME?X=

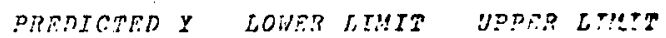

$3,3 ! 6$

297.131

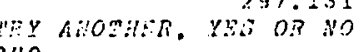

28.3 .821

310.440 
TRANSITION ANALYSIS: 6-5 YEAR TREND

DISTRICT 非, GREEN ISLAND (MIXED STRATUM)

GRADES $4-5 ; 5-6$

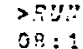

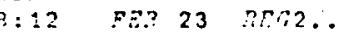

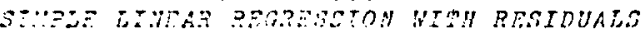

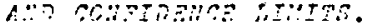

?5

YALUES Y.X FOR OBSERVITYOWS

$2: 210,: 34$

$\operatorname{lin}_{2}^{2} \leq 0,2: 2$

3

2205,255

$? 205,234$

5

$\because 299.234$

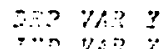

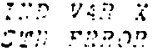

cospotsing

5emp?

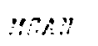

STAYDADD REYATTRB

123.500
233.800
27.9126

$27.5 ? 26$

241020

$5.201095-02$ 24.7447
38.4865

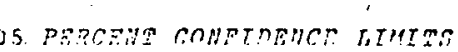
LOY:R yPPE?

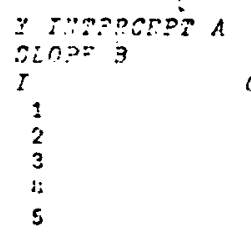

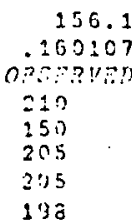

$-1214.175$

Fin? -383951

185.627

130.112

201.037
193.632

420.510

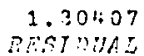

24.3733

$-40.2037$

3.36255
1.3693

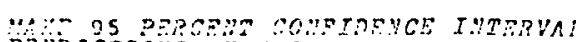

?YES

if? $P$ : $x=$

PRENTCTED Y LONER LTMIT

2297

T?Y AWOZHER, YSE 0 OP 319

121.391

UPPRR LIUTT
780 NALT

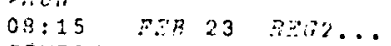

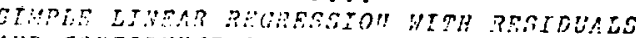

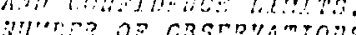

35

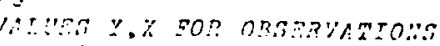

$\therefore 72,180$

205,210

3

$? 111,150$

$?+404.205$

$\frac{9}{5}+.205$

$? 114,205$

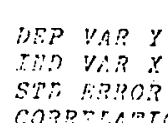

MEAL

113.200

190.2004

40.3254

CopresAron -.410340

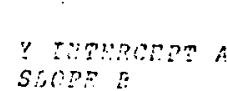

168872

Sollapis

594.058
3620.79

3003.79
54.00959

11. 3074

13.0732

$229 \% .32$

. $I$

1

1 (7)

$23: .571$ 6.23529

37

65

$1: 1$
104
114

114

TANDARD DEVTARTO

33.3126

25.2433

SAKE 95 PEPCEIT COHPTDEMCR IUMERVAL YRSRTETRIES, YES OR UIO

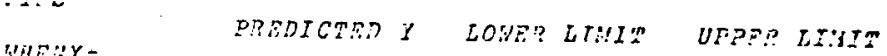

$? 204$

Tr? A.MOHER, y 204.471

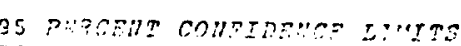

$-254.571$

$-3.1 \sin 81$

119.435

$\because 17.912$

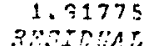

5?.50.47

$-35.7234$

$-27.1412$

20.1523

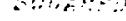

2753.05

2.75 .59

735.643

$2.33910=-02$

123.022

4379.33

?NO 
TRANSITION ANALYSIS : 6- 5 YEAR TREND

DISTRICT \#2, GREEN ISLAND (MIXED STRATUM)

GRADES $6-7 ; 7-8$

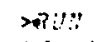

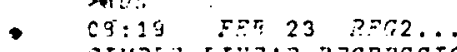

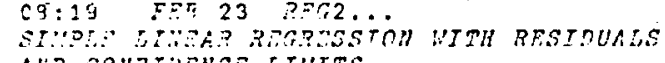

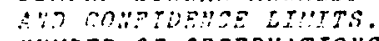

$3:$

OLEVE Y X FOR OBATRUTIOHS

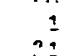

$? 215,172$

$? 213.05$

4322,111

5

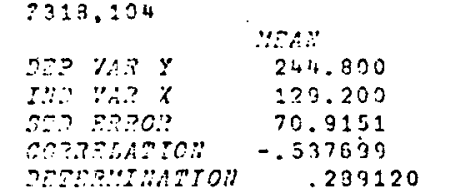

STAPBARD DEVTATEOH

72.8402

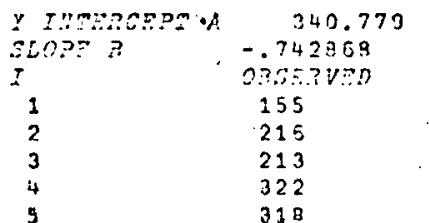

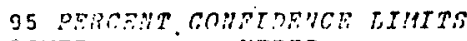

HCWR? UPDE?

$46.4521 \quad 635.125$

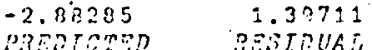

iventrots

196.662

292.492

258.320

20.3 .520

$-41.6522$

2.99475

$-79.1921$

54.4737

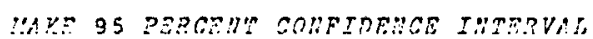

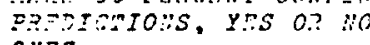

$? Y 23$

PREIETSD Y LOUER LTHIT UPREF LIMTT

:.::::XX

$149.100 \quad 377.940$.

T.P A.ROTHER, YES OR 263.520 $\because \because 0$
$>$ PU:?

$03: 22$ Rr? 23 REC2.

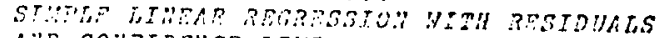

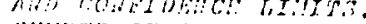

$? 5$

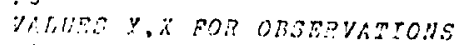

? 161,240

2
$? 243,155$

$? \frac{1}{3} 43,1.55$

$? 149.2: 6$

$? 270,213$

$? 312,322$

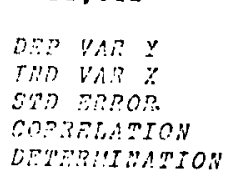

ma:

211
53.4500

53.4500
.807221

7月,

70.4238
59.5152

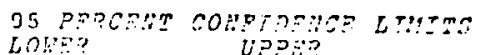

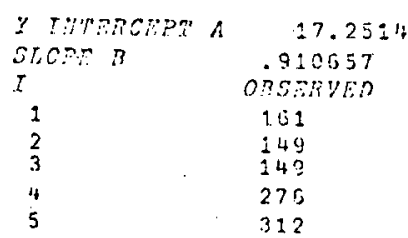$$
.551605
$$

SחVA.T.
1735.74
$B .96851$

8.36351

6319.00

4055.12
2368.04

15086.3

OrSTEVD
1.11
149
149
275
312

$-251.841$

$-.322854$

pisingen

152.339

158.403
213.953

211.221

310.453

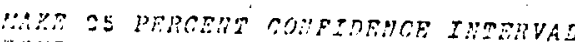

Minicilols yes or No

?yist

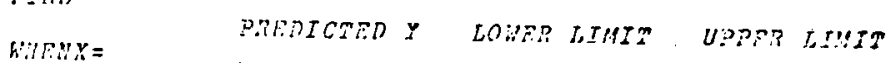

2253

TRY AHOTHER, YSS OR 250

157.598

355.920 
TRANSITION ANALYSIS: 6-5 YEAR TREND DISTRICT 非, GREEN ISLAND (MIXED STRATUM) GRADES 8-9

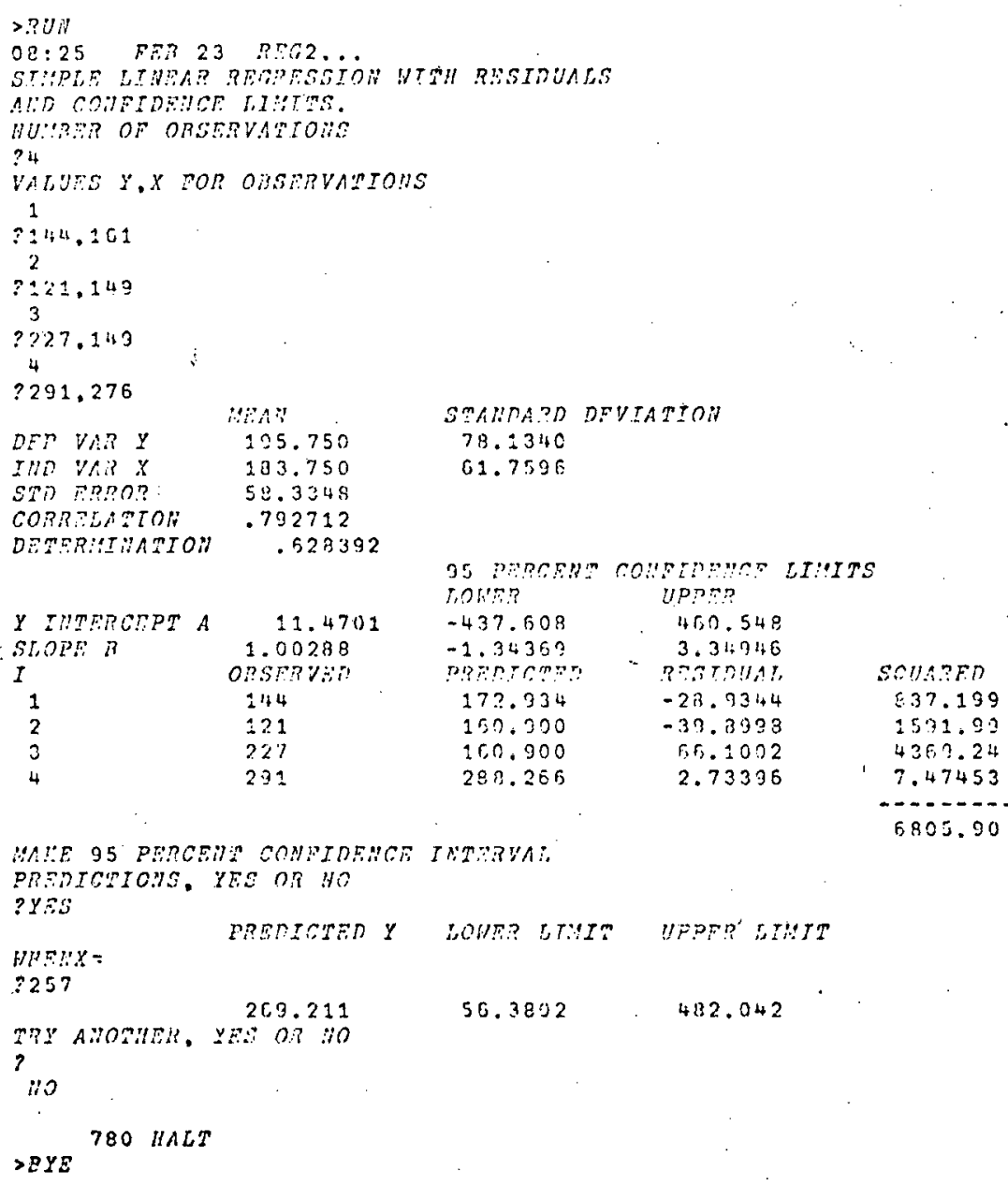

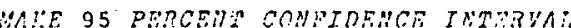

PREDICITOHS, TES OT HO

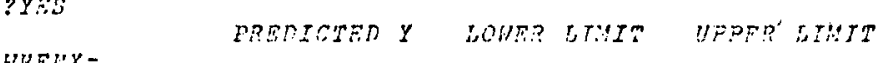

He.?:X

$203.211 \quad 56.3832 \quad 482.042$

MPY ANOAYRE, Y 263.211

56.3802

482.042

\%

$>E Y E$

780 HALT 
TRANSITION ANALYSIS: 6-5 YEAR TREND

DISTRICT 非, RIVERSIDE-DIAS (MIXED STRATUM)

BIRTH-GRADE 1; GRADES 1-2

17:43 TER 22 RES2.

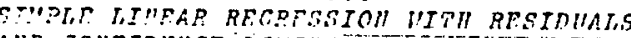

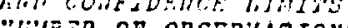

WLUES $Y, \bar{X}$ FOR ORSGRVATIOUS

$? 273,2214$

$\because 271,222$

32.222

42.256

$? 273,226$

25

$? 25,0,178$

$? 28 \div, 242$

Bif

Ii:D VA? $Y$

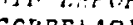

CCEDERATR"

"PAll STALIARD DEVIATIOH

$279.167 \quad 17.6115$

224.667

26.3413

95 PFPCFM" COHFINFHCE LIMITS

\begin{tabular}{|c|c|c|c|}
\hline \multirow{2}{*}{\multicolumn{4}{|c|}{ 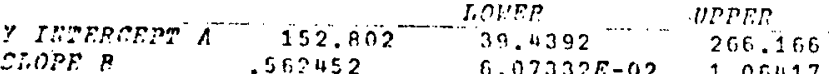 }} \\
\hline & & & $26 \in, 166$ \\
\hline SiODF $P$ & $\begin{array}{l}.562452 \\
\text {. }\end{array}$ & $6.073325-02$ & $\begin{array}{l}1.00+17 \\
0\end{array}$ \\
\hline 1 & $\begin{array}{l}273 \\
27\end{array}$ & $\begin{array}{c}\text { PlPENCREA } \\
278.792\end{array}$ & $\begin{array}{r}\text { RFETHERL } \\
.208301\end{array}$ \\
\hline 2 & 271 & 277.657 & -6.RfG \\
\hline . & 312 & 296.790 & 15.2008 \\
\hline 4 & 273 & 279.917 & -0.9160 .0 \\
\hline 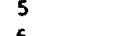 & 26.0 & 252.919 & 7.08109 \\
\hline 6 & 280 & 288.916 & -8.91583 \\
\hline
\end{tabular}

MALE 95 PERCEIT SOIFINTHEF TITFPVAL

PDPOICTIOHS, YRS GP 110

?:Pat

PREDTETRY LOHPR LTHTT UTPRR LIUTT

PREPY:

284.416

271.475

297.358
750 HIA I.

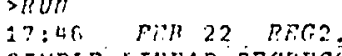

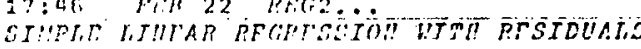

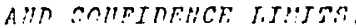

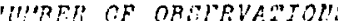

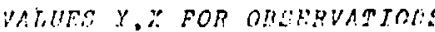

2262,279

$\stackrel{2}{? 276,271}$

3

$? 263,312$

2283,273

5
203,260

DRP $V A R Y$

(:T) Fr?:OR

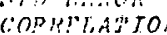

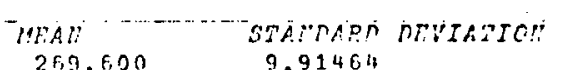

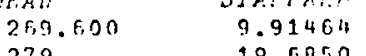
9.9146050

10.9403

8. $67981 F-02$

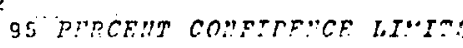

Y IMTERCRPm

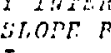

$I$

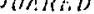

1. $338948-02$

411.4452

231.339

47.3594

50.2418

79.4921

$453.30 ?$

HAKE 95 TERCER'S COMPIDEHCE IMTERVAL

PRTDICTTOIIS, YRS OR NO

?YPS

ritinTCSTD $Y$

LOWER LIUI?

HIl $F$ ? $Y=$

2284

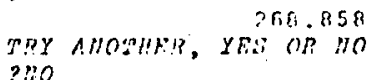

252.0 .74

285.042 
TRANSITION ANALYSIS: 6-5 YEAR TREND

DISTRICT 非, RIVERSIDE-DIAS (MIXED STRATUM)

GRADES 2-3; 3-4

17:49 ELB 22 REC2:

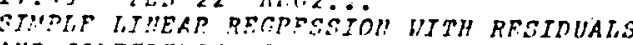

Alln COl'FIn WC LI"IS:I.

$\because 3$

?':

WADUTS Y,Y FOP ORGEPVATIOHS

2270,278

2270,262

2218,276

218,276

$? 288,263$

5

$? 238.294^{\circ}$

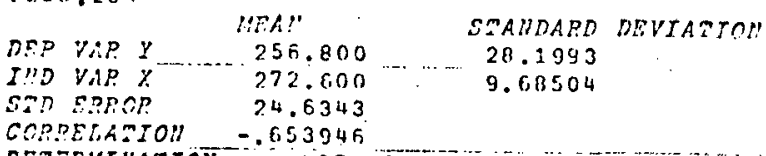

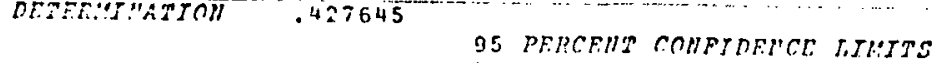

\begin{tabular}{|c|c|c|c|}
\hline Y IUMERCRPR A & 775.844 & $\begin{array}{l}L C \lg R \\
-327.863\end{array}$ & IFPFP \\
\hline$\therefore 20 ?$ & -2.90405 & -5.05083 & $\begin{array}{l}1079.55 \\
2.111272\end{array}$ \\
\hline & OPGEPVFD & PPENYCOFE & $R E S P D U A$ \\
\hline 1 & 270 & 240.518 & 23.4219 \\
\hline & 270 & 276.983 & -6.983014 \\
\hline 3 & 218 & 250.326 & $-32: 325.2$ \\
\hline & 288 & 2.75 .079 & 12.0211 \\
\hline 5 & 238 & 235.094 & $2.9 \cap B 1 A$ \\
\hline
\end{tabular}

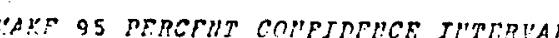

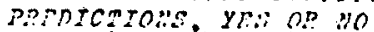

166.055
8.44500
-14.025

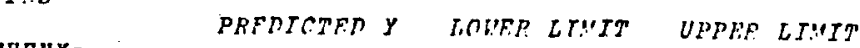
3283

263.655

?:0
$225.692 \quad 301.6 \div 7$
STIATER

48.7615

2044.98

1820.55
RUin

17:51 FFH 22 RFF?

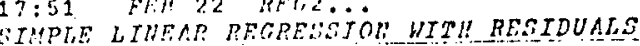

AHI COHFSNEMCE RIT'TAS.

MIIITER OF ORELPVIATIOHS

PA

1

$? 21 \%, 270$

$2 \div 31,270$

$\because 230.212$

? 282,288

DTS VAB $Y$

In: ba? $\mathrm{X}$

antingoR

COTRERATIOH

DETPRITINATSOM

WFA? _... STAUDAPD DEVTAETO?

$247.400 \quad 21.0329$

18.3506

28.9137

risigif

.390521

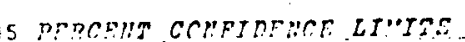

Y THTERSETT A

STOPE $B$

$I_{1}$

2
3

4

126.036
4545.65
$\sin \sin 250$
247
247
231
230
232

LOIE: ipros

$-153.008 \quad 405.079$

PDETCro -

257.400 Perrolus

257.400 $-10.6000$

240.784

-1775354

225.127

4.87273

256.945 250.0545

1010 $3: 5,5+7$ 23.9435 E 27.730 1078.29

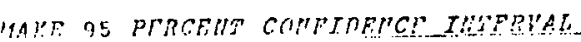

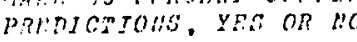

?YES

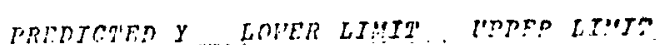

WIIFIY =

2264

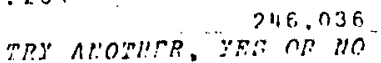

$218.87 ?$

273.196

?tio 
TRANSITION ANALYSIS: 6-5 YEAR TREND

DISTRICT 非, RIVERSIDE-DIAS (MIXED STRATUM)

GRADES $4-5 ; 5-6$

$>$ Pli?

$17: 54 \ldots F^{r} R 22 \quad B R C 2$

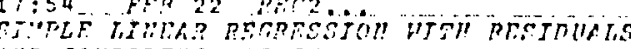

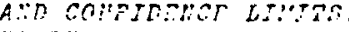

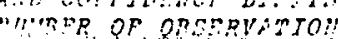

"ALEES Y,X TOP ORERPVATIOP'S

$? 223.293$

2236.247

$? 179,247$

4

$? 153,231$

$\stackrel{5}{2} \div 5,9.230$

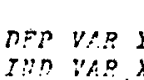

MTARI:

191.200

STAPDARD DEVTATYOI

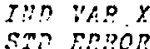

229.500

35.9263

contritarion $40.5 \% 71$

DETERIIHATSH -.207970

95 PFRCTHT COMFTAFICE HIIUTS LOWF?

UTPEP

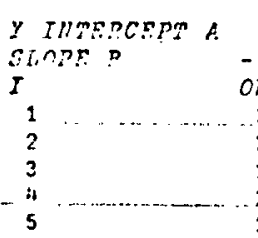

26
.334
$08 \div 5 \%$
223
235
173
153
168

$-404.737$ -3.25itcs 2.5873 PPFITIRR RFIIDUAT, $204.1914 \ldots 18.8058$ 185.900 135.908 191.005 $-5.9076$ -38.3250
$-23.6,645$

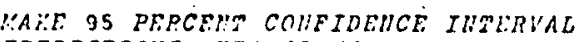
PDENICTIOIS, YRG OR NO

PRPRICSEN Y LOURR LIMIT UPPEP LIUIT

bestix

PY ANOAFER, YES OR I!O

111.167

201.326
17:50 TrR 22 RTC2..

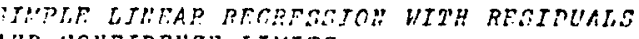

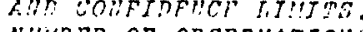

32

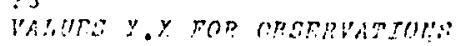

i 155,298

$\stackrel{2}{2} 131.223$

$21 \geq 8,236$

$? 143,179$

$? 132.153$

$D F D^{\prime} \quad V A R Y$

IIID VAR X

STD ERROR

CompLLAT IOU

Tenin

STAMAARA REVIATIN"

138
197.800

12.930

$6.07013 \%-02$

OS PEPCEHT CONFTREVE LINSE

reniaren

353.6 .57

2509.24

117.7158

1463.87

560.011

4939.50

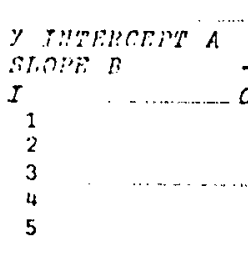

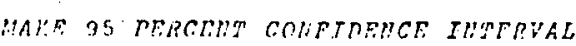

PRTDICSIOHA. YES OR nO

IINFUX = PRERIOTEE Y LOHFR LIMIT UPPER LIMTT

$1180 \quad 139.007 \quad 119.221 \quad 159.793$

ITPY AHOTHER, YES OR 10 
TRANSITION ANALYSIS: 6-5 YEAR TREND

DISTRICT 非, RIVERSIDE-DIAS (MIXED STRATUM)

GRADES $6-7 ; 7-8$

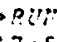

:7:59 FrR 22 PFr:

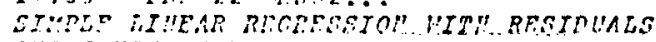

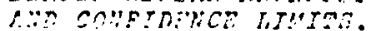

and of oncromentous

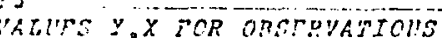

$\therefore 32.287$

$? 15 ?, 150$

$\because 93.131$

442.20

$?: 51,143$

TEP YRY $Y$

$\operatorname{Ian}, y=0$

at? ERpos

CCosinarion

TEmentitation

UTAR - STAMIARE REYIATIOH $\begin{array}{ll}170.400 & 34.5731 \\ 149 & 23.9479\end{array}$

140

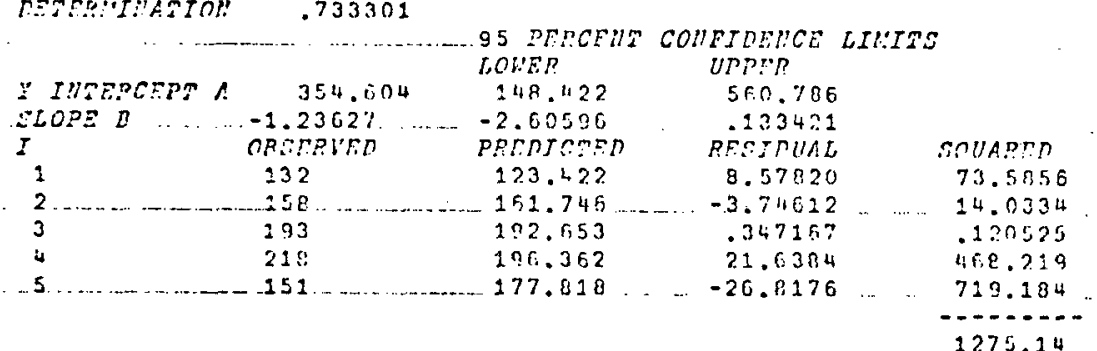

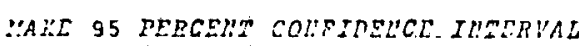

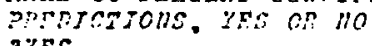

THF

PPEDICTED Y ... LOHER LIUIT . UPPER LIUIT

Wr: $x=$

2139

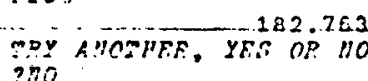

780 IALS

13:01 FRD 22 PRC2

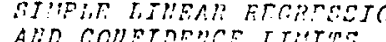

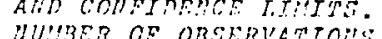

II)!

VALUES $Y, Y$ FOR OREEPVATTOUS

$? 234,13:$

?ं2, 132

$? 117,158$

$? 117,158$

P188:193

5
293,218

DFP VAR:

TWD VAR $\because$

CORn:LATS

DESERIITIATIOH

MEAR

$214.800 \quad 53.3774$

166.400

20013

$7.23398:-02$

35 PERCER' COHFIDENCE LI:TSS

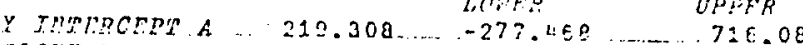

St.OPP $R$ - A

$T$

2

3
4
5

275.14

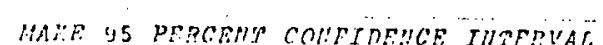

PRERTCTOH, YE: OR HO

?yEs

:PRn $=$

PREDTC'ED Y LOHTP LIIIT UPPEP LIMTT

$? 183$

TRY ANOZUER YES $13 \% 3 \pi \%$ 
TRANSITION ANALYSIS: 6-5 YEAR TREND

DISTRICT 非, RIVERSIDE-DIAS (MIXED STRATUM)

GRADES 8-9

$>$ RUII

780 HALT

$38: 04$

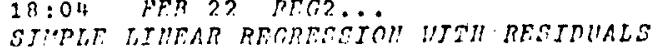

AIID COIFIDEUCE LIYTSE.

MII'MUR OF ORSEPVATIONG

$?$ ?

WALHSS Y.X FOR ORSERYATIOUS

$? 141,234$

? 93.92

$? 124,117$

4
$? 51.188$

Ding var

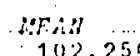

STALDARD DEVIATIOL

DEP VAR Y

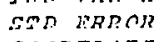

102.250
157.750 30.5253
65.0367

CORDELATTON .145908

CFIEPMIIIATOH $2.12801 \mathrm{C-O2}$

$$
2.12891 \Gamma-02
$$

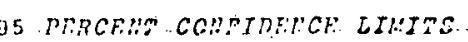

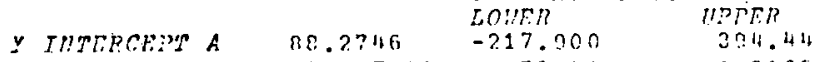

SLOPE B

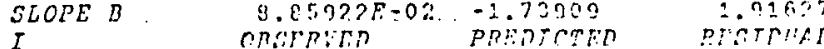

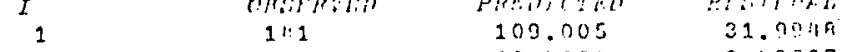

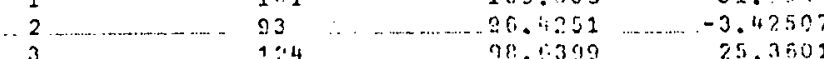

4

$51204.930 \quad-50.02009$

sellesen

$1023.6,7$

$11.7311 \ldots$

643.136

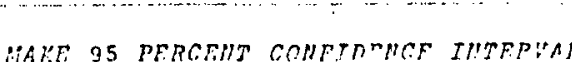

4586.97

TRENTCTIOLS, Y YES ON 110

?YRS

WIIRIX

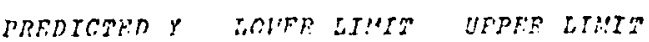

. 137

gRY AHOTHER, YES OO 112

$-9.39210$

210.206

?IIO

$>O F F$ 
APPENDIX F 6

TRANSITION ANALYSIS

EIGHT - SEVEN .YEAR TREND

PARISH OF HANOVER 
TRANSITION ANALYSIS: 8-7 YEAR TREND

PARISH OF HANOVER

BIRTH-GRADE 1; GRADES 1-2

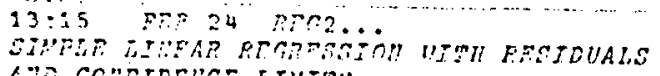

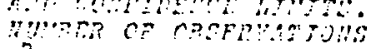

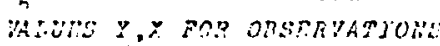

i

$\div 030.2352$

$? 0000.2339$

2234,2320

$22435.2222^{3}$

5

$-?$

$.22 \div 2.2128$

$733: 8.2193$

8

$? 2341.207 \%$.

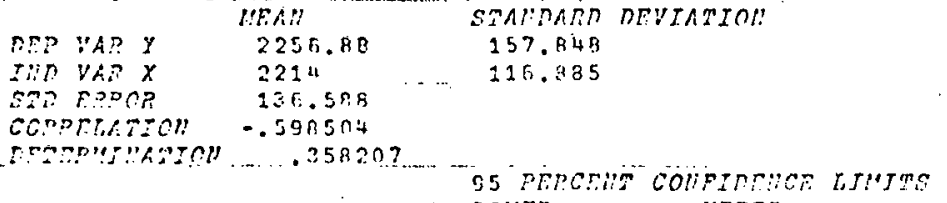

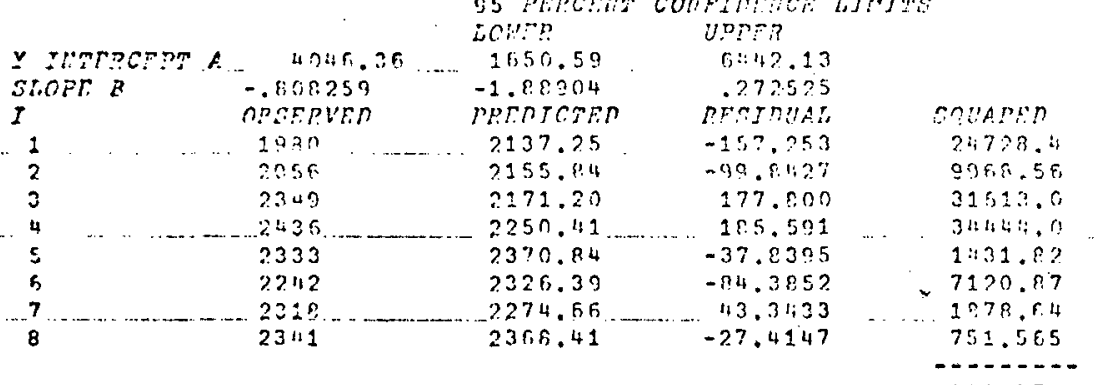

"AY 55 PEPCEYT COUPIDEDCE IIITIRVAL

DofEICAIOUS, YES ON 110

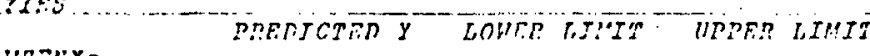

$\operatorname{ros} x=$

121037

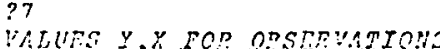

31050.1000

5

$? 2004.2056$

22118.2349

$? 3313.2436$

5

5

$? 2193,224 \%$

2.2.14.2319

$\lim$ ing

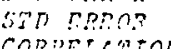

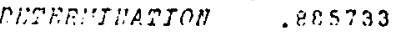

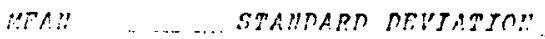

$2124 \quad \quad 175.176$

0 की 113 iा

$\begin{array}{llll}\text { YHITHRCEPA A } & -30.8592 & -1019.02 & 821.204\end{array}$

581270 - 1.00013

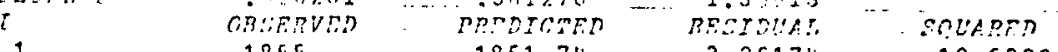

$2 \quad 1865 \quad 1851.74 \quad 3.26174 \quad 10.8390$

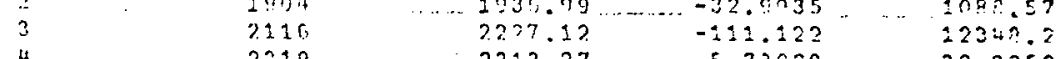

5 3257 32732.835

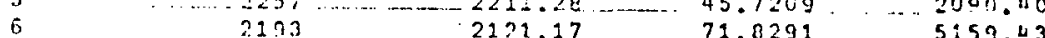

$2193 \quad 2131.17 \quad 71.8291 \quad 5159.43$

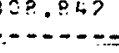

MAKE 35 PRPCENT COUFIDIMCE INTERVE

21033.9

PREDTCTIOHS, YE⿱ PYFS

$\because n E N \times=$

PRPDECAED Y LOMER LIUIE UPPER LIIITT

2.60

$2146.92 \quad 2083.17 \quad 2210.66$

AHOSIISP, YES OR

2210.66

TPY AlOTHER yes 2258.1

32 
TRANSITION ANALYSIS: 8-7 YEAR TREND

PARISH OF HANOVER

GRADES $2-3 ; 3-4$

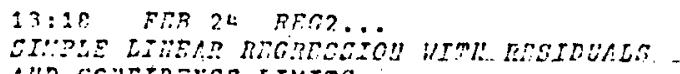

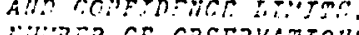

27

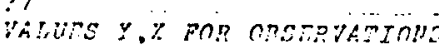

1

तิ2:4.1286

21933.1905

$-3$

2002.19004

$? 2157.2116$

$? 2250,23: 9$

$? 2272.2257$

$22160.2133 \ldots$

\begin{tabular}{|c|c|c|c|}
\hline & & & \\
\hline DTR YAR $y$ & 2087.71 & 175.1124 & \\
\hline 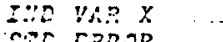 & $2017.14 \ldots$ & 190.305 & \\
\hline 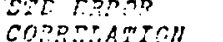 & 35.3095 & & \\
\hline "ER:YIMAZOU & $\begin{array}{r}.981004 \\
.05413\end{array}$ & $\ldots$ & \\
\hline & & $\begin{array}{l}95 \text { UFRCEIIT } \\
\text { GOWR }\end{array}$ & 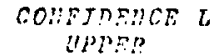 \\
\hline I:UERCEPA A. & 221.92 & $\ldots-205.546$ & $0,29,38 \mathrm{fi}$ \\
\hline$p=3$ & .203065 & .702003 & 1.13332 \\
\hline & CFSFBy & PREMTSRD & RESTDUAT \\
\hline & $191 \%$ & 1915.10 & -1.00094 \\
\hline & 2032 & 2000.24 & $36.80,44$ \\
\hline & $10: 2$ & 2932.20 & -49.3551 \\
\hline & 2157. & $2122.80 \ldots .$. & $3 ! .1952$ \\
\hline & 2296 & 2305.13 & -10.1289 \\
\hline & 2272 & 2230.14 & $21 . t 0.31$ \\
\hline & & 2192.34 & \\
\hline
\end{tabular}

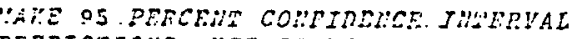

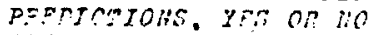

?UES

$m ! m x=$

?214?

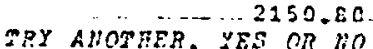

PRERTETR Y LONES LIHIT . LPPER LIUIT $? \pi 0$

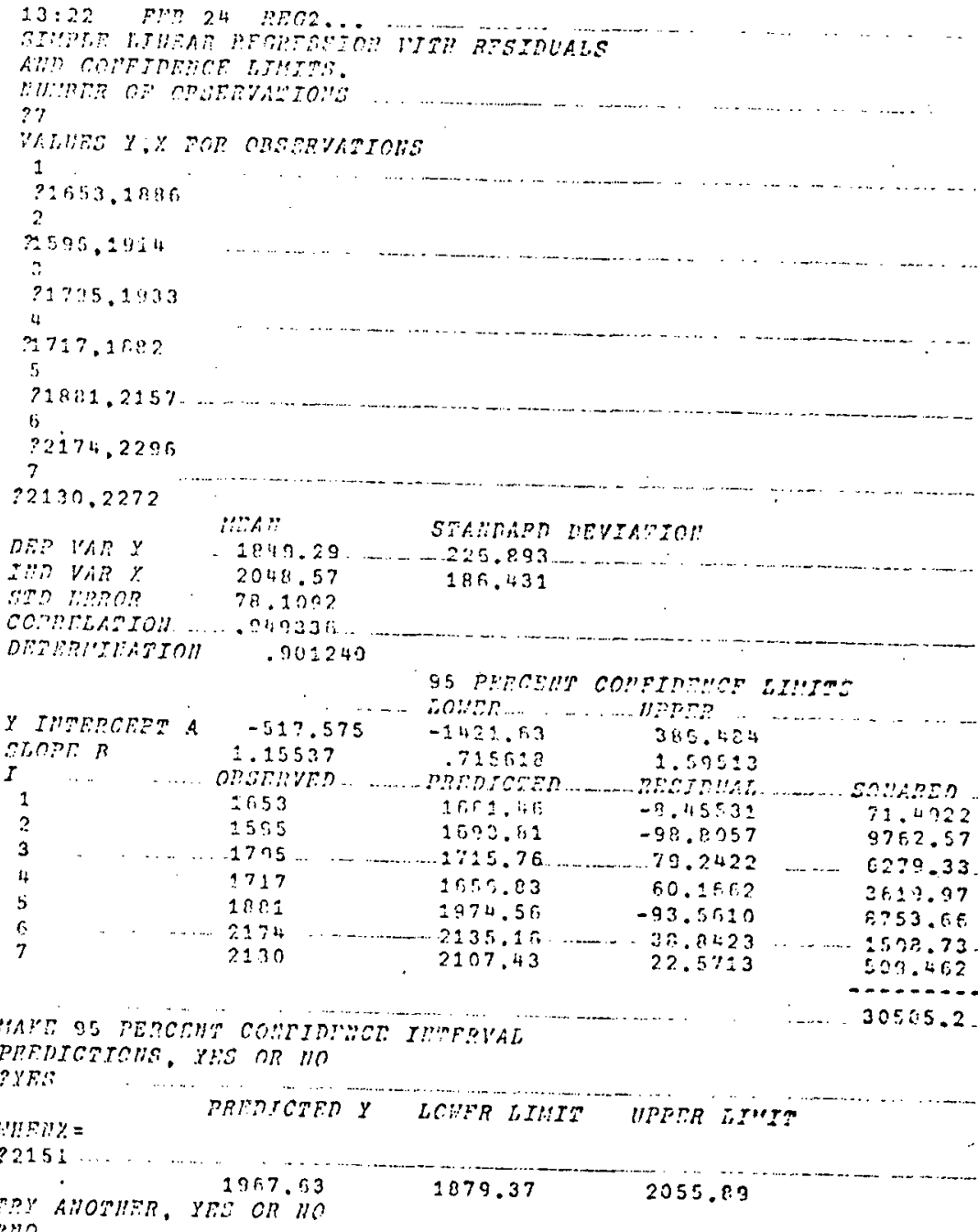

TOY AHORMER, YRE OR 196 
TRANSITION ANALYSIS: 8-7 YEAR TREND

PARISH OF HANOVER

$13: 25$ FED 24 RKG $2 \ldots$

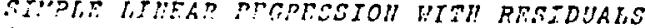

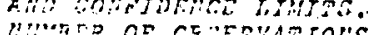

?7

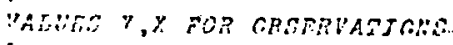

$? \div 530.1624$

$? ? 255,1053$

3

$?: 319,1595$

$? 2443.1795$

$? 1434.9727$

$? 1531,1891$

$? 2797,2174$

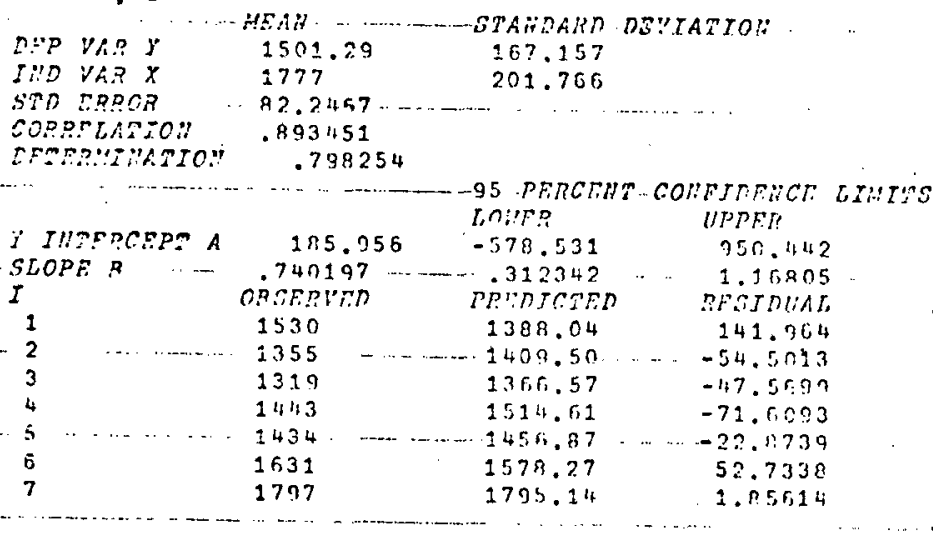

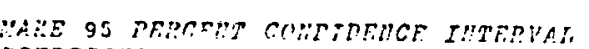

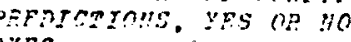

prentegron $y$

LOWRT LITIS

(IM):P EIIIZ

$2196 \%$

TPY AHOTPER, $\quad 1642.65$

1528.38

1755.07

\section{GRADES $4-5 ; 5-6$}

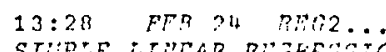

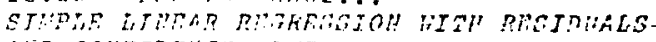

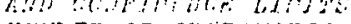

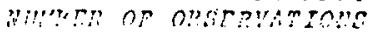

37

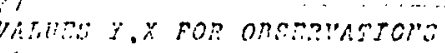

?1367,1240

2

$n_{i n ? 7.1530}$

$? 7667.2355$

2222.1329

5

0010.1443

$070,1+34$

$? 2029,1631$

\begin{tabular}{l}
$n$ \\
$I$ \\
$C$ \\
0 \\
$y$ \\
$I$ \\
\hline
\end{tabular}

REF VRP

IIIT VAR

STh RePRos

cononiusuriton

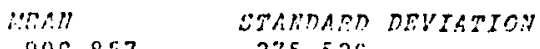

$\begin{array}{cc}900.857 & 275.520 \\ 1121.71 & \end{array}$

277.57

1. ... . . . ....

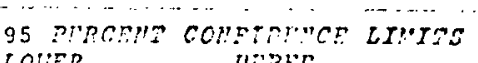

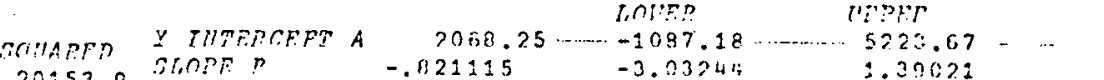

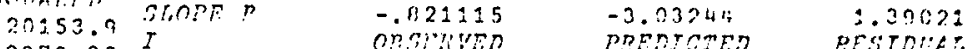

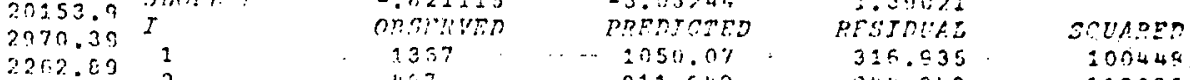

$5: 27.03$

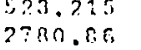

3.114526

33822.6

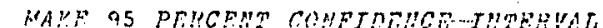
$\begin{array}{lrrrr}1357 & 1050.0 \% & 315.035 & 100440 \\ 1167 & & 211.042 & -341.042 & 110005\end{array}$ $\begin{array}{lrrrr}1357 & 1050.0 \% & 315.935 & 100446 \\ 1167 & 211.042 & -344.042 & 110005\end{array}$ $\begin{array}{lrrrr}1357 & \cdots & 1050.0 \% & 315.035 & 100440 \\ 1167 & & 211.042 & -341.042 & 110005\end{array}$ $76.7 \quad 055.637 \quad-100.53 \%$ 35.0.0

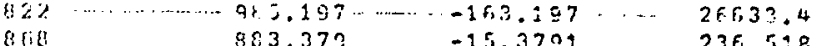
976 $\quad 83.370-15.3701 \quad 236.528$

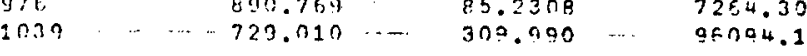
385245

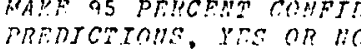

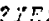

WItany=

21643

PPY AMOTHER, YFE OI? 719

$? 1 / 0$ 


\section{TRANSITION ANALYSIS: 8-7 YEAR TREND \\ PARISH OF HANOVER \\ GRADES $6-7 ; 7-8$}

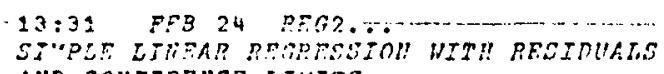

A.D COHETRECR WI:ITS.

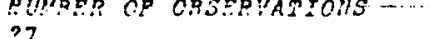

YALITE Y X EOR OROEPVATIOUS

21 993,1363

2

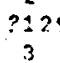

$? 1550,1467$

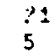

6

2177.858

$21: 01.976$

DRP VAR Y MEAL $\quad$ STAIRARD REVTATIO

IIV VARY $\quad 047.143 \quad 325.311$

Conogiancon 50.149

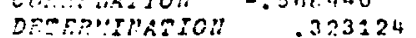

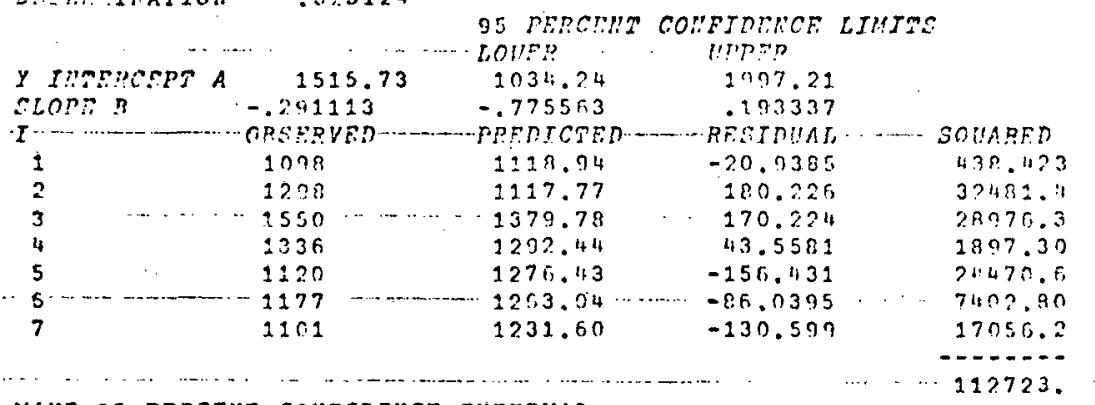

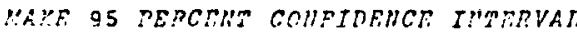

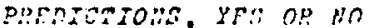

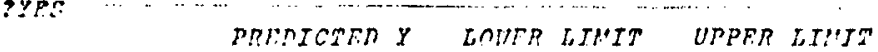

$\min m i x=$

12723

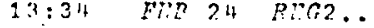

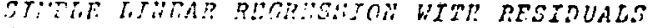

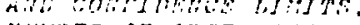

$? 7$

VALUR: Y.X FO? ORERVITTOHS

$? 1258,1154$

?ंग 017,1098

$? 1133,1293$

$? 484,1550$

336,1336

$? 659.1120$

$? 7,70,1177$

b.: in? $y$

Int in?

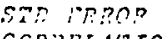

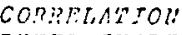

ERTERIIIIATSOH

$\ln 20$

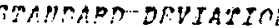

$205.96,0$

928
$124 ?$ 159.385

222.374

Y IHTKRCEPA A$$
\text { (2. } 051
$$

$2.0546445-02$
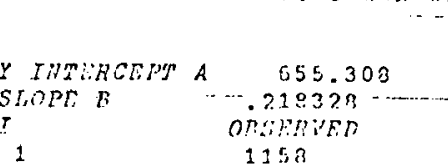

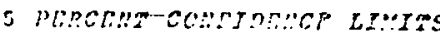
Lniti? 1180 us utr?

1

?

3

\section{MAYR 35 RERCEUT COMFINAICE IUTERIAL} $1.24503 \ldots \ldots+2169207$

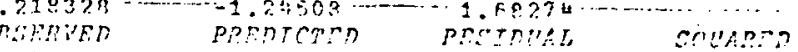

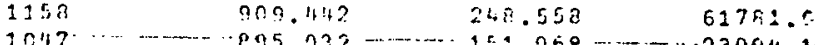

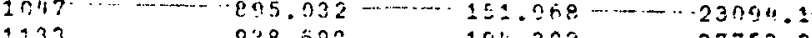
$1133 \quad 33.6192 \quad 194.302 \quad 3775.2$ $\begin{array}{lll}0.74037 & -9.71675 & 94.4152\end{array}$

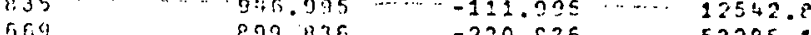
$670 \quad-230.236 \quad 5.205 .1$ 50699.8 247250 .

$\ln 8 \pi x=$ PREDTCERT Y LOWFT LIHTH

UPREP LI:!I:

708.792

$\$ 172.10$ 
TRANSITION ANALYSIS: · 8-7 YEAR TREND

PARISH OF HANOVER

GRADES 8-9

13:37 FFB 24 REC2..

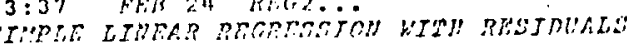

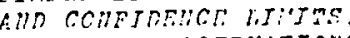

MHBER OF GRETRVATTONS

$? 8$

VALUNS Y, Y TOR CRSHRVATIONS

2974,1158

2

$?\{18.1047$

3

$? 733.1133$

2544,984

$x^{5}+40,835$

3523.609

InD VAR $X$

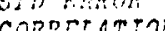

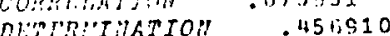

"MA"

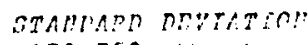

170.753

Y IIITERCEPT A

705.332

971.000

0.4510

109.429

95

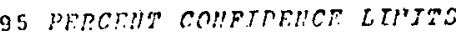

SLOPF $D$

$\begin{array}{rrr}109.423 & -805.308 & 1025.17 \\ .013701 & -.314080 & 1.54238\end{array}$

Tot?h
-805.308

UPPR?

2

974

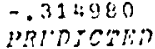

1.54238
RE:OTDUAL

$82: 1$ 0 55

751.975

804.753

713.311

52.870

519.996

f.6. 0254

$-150.311$

$-81,0509$

540
623

...

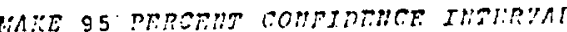

pornTCOJOIS, YES OR VO

PYES

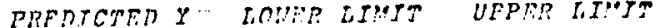

WISIIX $=$

7940

686.309

524.288

848.329

TRY AMOTHER, YRS OR HO 
APPENDIX F7

TRANSITION ANALYSIS

SIX - FIVE YEAR TREND

PARISH OF HANOVER 


\section{TRANSITION ANALYSIS: 6-5 YEAR TREND PARISH OF HANOVER \\ BIRTH-GRADE 1; GRADES 1-2}

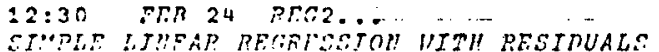

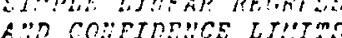

A.:.

$? 6$

TALOTE Y Y FOR OREEDVATTOUS

$? \pm 980.2362$

2

$$
3
$$

$? 2349,2320$

$? 24.36,2222$

22333.2072

$? 2242,2128$

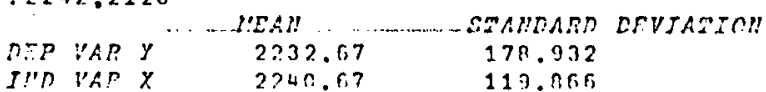

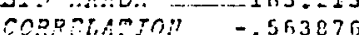

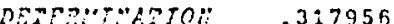

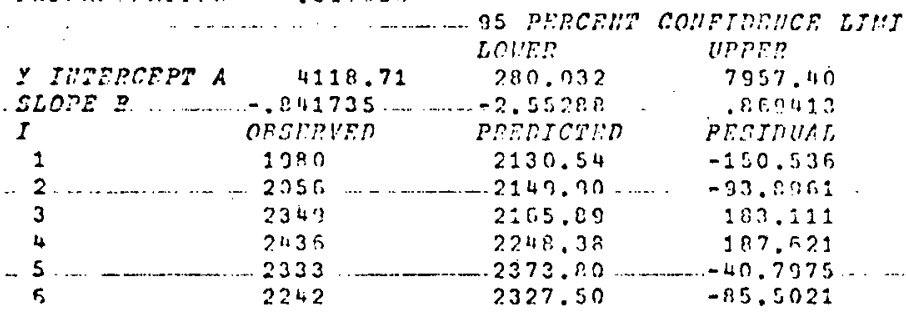

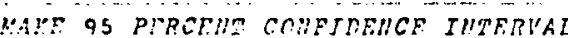
PREDICTIOHS, YFS OR HO

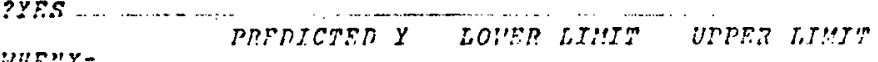

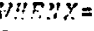

2466,02

moy allother, yrs or 22650 2067.15

2466.05

2710

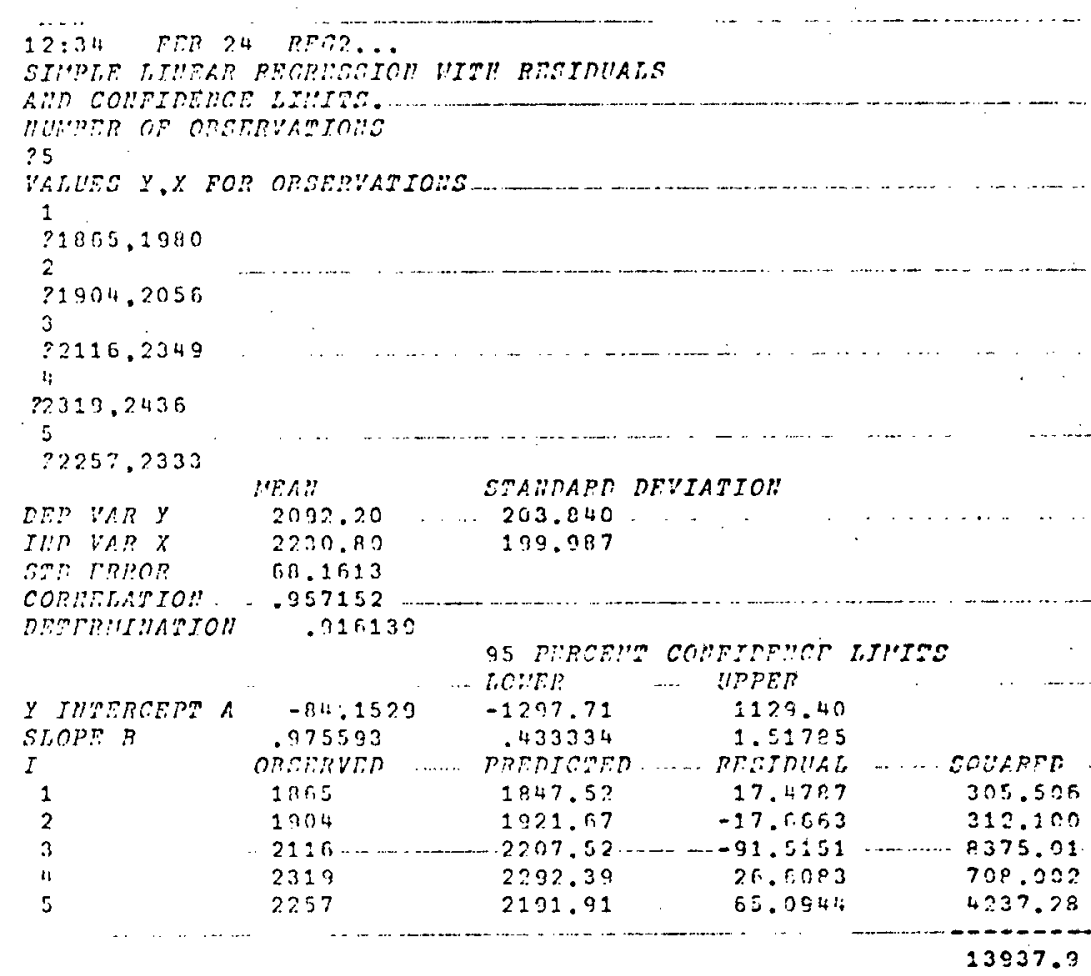

PRERTCTIOH:, YF. OR 110

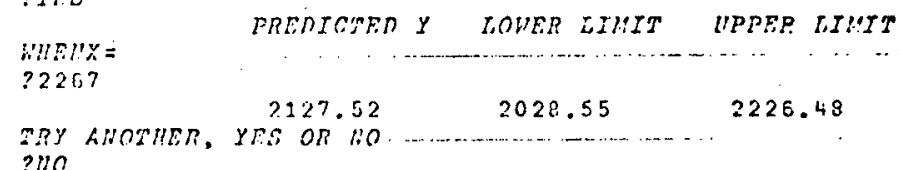

MAIF 35 PERCEUT COUFIREULE INGTRVAL

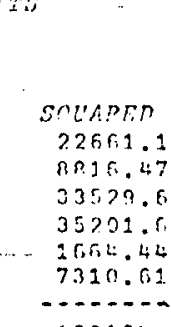


TRANSITION ANALYSIS: 6-5 YEAR TREND

PARISH OF HANOVER

GRADES $2-3 ; 3-4$

:2:37 FrR 24 prea2.

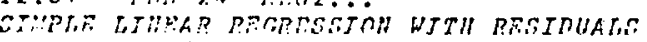

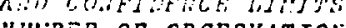

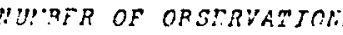

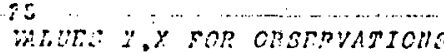

1

71914.2890

2

$? 933.1865$

?. 2362,1804

4

$? 2157,2116$

.2290 .2319

DEP :A? $X$

I:A

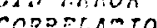

cons.cheson

TRAN - STANDARD DE:TATIOH

$2036.40 \quad 181.280$

1389.000

214.962

794254

.988541

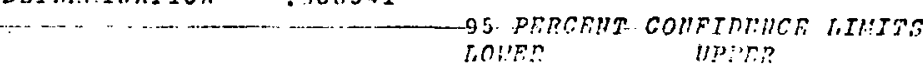

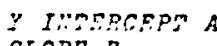

I

1

1
2
3

4

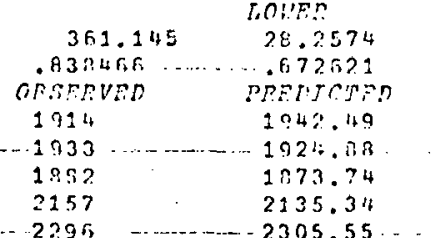

$\cos , 033$

1. กrius

RESTIUAL

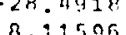

8.1150

29.100

$-9.54754$

RAXR 95 PRRCRHT COHFIDRNGE THTERVAI

POEOTCETOUS, TT: OR VO

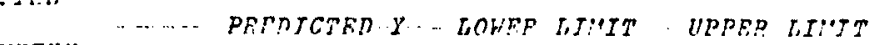

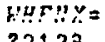

RY ANOTHER, YH'S 2145.40

$2106,91 \ldots \ldots . .2183 .89$

?!I0

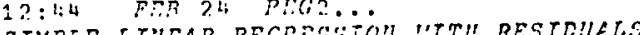

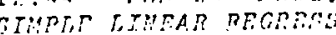

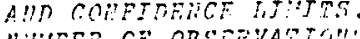

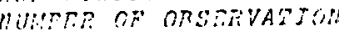

$? 5$

YALUES Y,X FOR ORERPVATYOHS

? 1652,1625

$2: 353.1314$

3

$? 1795,1933$

$? 1717.1882$

11981,2157

DRP WAS $y$

In VAR $X$

$\sin$ bes?

COPBETARTON:

STALDARD DTVIATYOH

$1728.20 \cdots+113.284$

DETRMLIIITTOH

81.35143

115.171

(2)

95 PFRCFYT CONFIARECE LIVITE

y IIISERCRPT 4 $222.960-1976.81 \quad-19 P F P$ $\begin{array}{rrr}222.960 & -1976.81 & 2422.73 \\ 270100 & -.353009 & 1.20417\end{array}$ $.770180 \quad-.352809 \quad 1.00417$

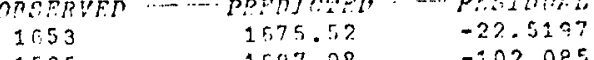
10,53

SCURTER 811.784 $6,5.8688$
$6,26,09$

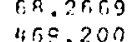
01.1556 $\frac{01.1556}{1906.28}$

1506.28

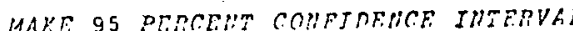

$\begin{array}{llll}1597.08 & -102.085 & 507.136 \\ 1505 & 1697.0821 .3\end{array}$

$8.2819-535.97$
4.5690

$1801 \quad 2804.24 \quad-3.23849$ 12325.59 10.4873 19860.5 PREOICOIONS YHe: no HO

?YT.

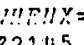

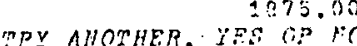

1631.48

2118.52 
TRANSITION ANALYSIS: $6-5$ YEAR TREND

PARISH OF HANOVER

GRADES $4-5 ; 5-6$

12:46 FrP 24 arrez..

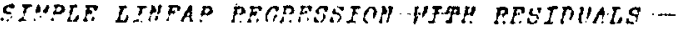

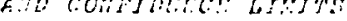

YAD:ES Y.X FOP OREEPYAmYOLA

$\because \geq 530.1624$

2

$? 1355,3653$

13.

71319.1595

$?: 1443.2705$

21434.1727

\begin{tabular}{|c|c|c|}
\hline DP VAR Y & $142 \pi, 20$ & 024300 \\
\hline IHD VAR $X$ & 1676.80 & 80.0512 \\
\hline ppon & 91.4876 & \\
\hline
\end{tabular}

sersing

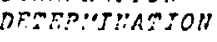

$7.61200 E-02$

y IP:PRCFPa A

939.826

95 PFRCEI'M COMPIDENCE II"ITS itrope

$r$

1.

1530

2355

131

1443
1434

LOI'ER

$-2111.87$ $-1.531: 20$
PPEDTCERD PPERTCED
1101.20

1409.4

1372.95

1427.62

UPPER

3992.52

2.10239
Pristint

12ก. กก

$-54.4335$

-73 . 7 ring

$-6.78026$

6.37930

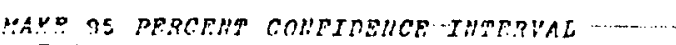

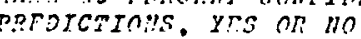

:I:E::Z=

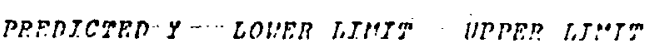

21875

TRY ANOTHER, YES OR 110

$1089.33+\cdots \cdots+1855.69$

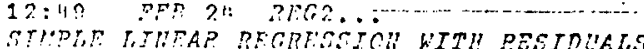

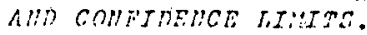

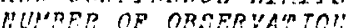

32

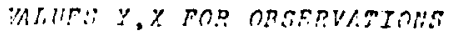

1

23567.1240

$?$ ?ी $8,7,1530$

2707.1355

222,1319

50.1443

DTS

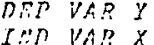

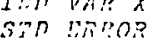

Corertatron

DFTERMIMATIO

IEA:" STAMAPD DRYTAMTOI

$858.200 \quad 324.659$

1377.019

324.659
112.2223

.8 .50 .313

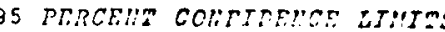

$Y$ JWERCEPT A CLOPE $P$

1

SOMARER

1.5 .589 .5

205.2 .55

5470.21

45.771 ?

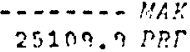

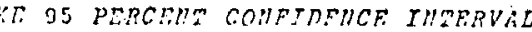

PYRE

WI:FIX $=$

$? 1473$

renorot

LORFR LIUIT UPRER EIUT?

TRY ANOMURR. YE: $0.37 \%$

240.891

1001.86

?110

TOLER TREP

$79500-$
-5.22206 PREDTCER DERTE

1178.57

480.173

$\begin{array}{ll}-12.1729 & 22357.7 \\ -146.590 & 173.526\end{array}$

$-146.500 \quad 2: 518.3$
-190.079

$172.307 \quad 32717.3$

1......

(1)

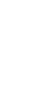

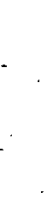




\section{TRANSITION ANALYSIS: 6-5 YEAR TREND PARISH OF HANOVER}

GRADES $6-7 ; 7-8$

12:52 FFP 24 REC?...

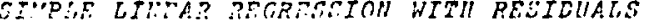

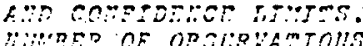

Muspre of Oraliovaminlis

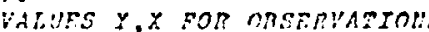

1

1008.1363

$? 2298,1367$

$? 2550,457$

$? 1336.767$

$? 1220.322$

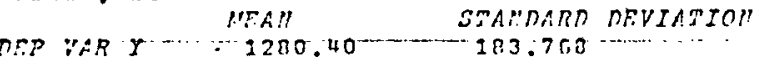

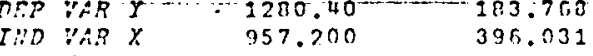

Sen EPSOR 153.105

CORRETA-TOR - - -.645294

DERERTYYTIOH $\quad .416404$

25 TERCFHA COHFITINCF LT:'JTO

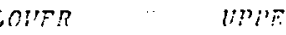

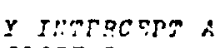

$156 \% .02$ 302.341

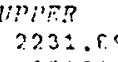
SLOPF $B$

i

:

2 4

1098
1259
155

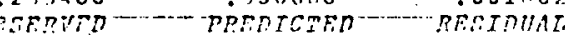
$1098 \quad 1158.89 \quad-60.890 ?$

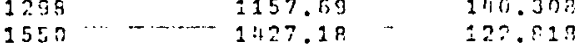

$13362 \quad 1337.35 \quad-135212$

$\begin{array}{lll}1120 & 1320.88 & -1.30 \\ & & -200.883\end{array}$

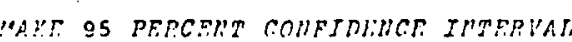

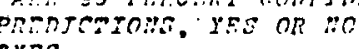
Prenjaten y

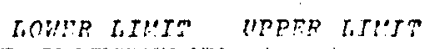
$\because R E P X=$

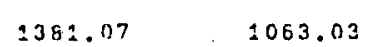
2699.11

1381.07 1063.03 210

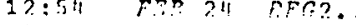

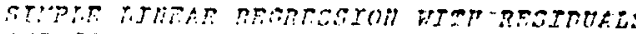

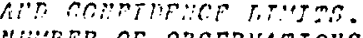

?

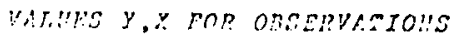

$.215 \pi .2104$

$? 1047.1998$

$? 1233.1230$

?3द4.1550

3035.1335

DEP VAR Y

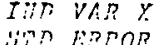

CORmLATYO

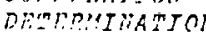

MAll STAMRARD REVIATIO:

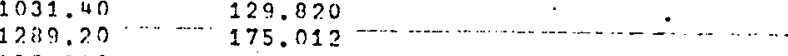
135.010

$-434553$

.10 .8837

o5 PRPCEIM COHFPDAHCR LIUITS

LOHFP. UTMT?

I ThESRCERT A

$\operatorname{SEOPCB} B$

EnIARTR

3707.61

10506.2

15024.2

$1.8202 ?$

40354.1

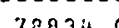

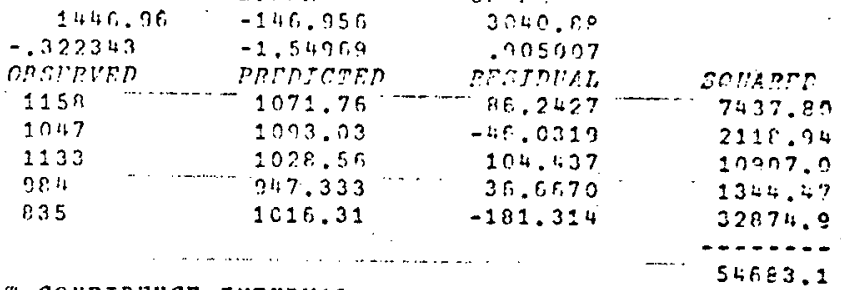

MALE 95 PRPCEHT COHFIDEUCR IUTERVAL PREDTCTOLS, YE: OP 110

\begin{tabular}{|c|c|c|c|}
\hline Mnsmx $=$ & SRIDTCRED Y & LOW':P LIIIT & UPPEP ATIIT \\
\hline $\begin{array}{l}\text { ?3381 } \\
\text { TRY ANCTHRR }\end{array}$ & $\begin{array}{l}1001 \cdot 81 \\
\text { YR: OR W0 }\end{array}$ & 779.084 & 2224.53 \\
\hline
\end{tabular}




\section{TRANSITION ANALYSIS: 6-5 YEAR TREND PARISH OF HANOVER \\ GRADES 8-9}

$>$ reut:

$13: 00 \quad 5 r R \quad 24 \quad 051 \% 2$

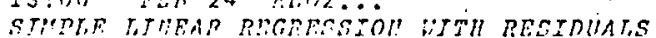

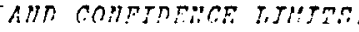

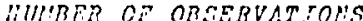

$? 4$

Writr: Y, For on ER?

1

5

Q 18,1047

-.

is

DEP VIS

IIID VAR $X$

33.920

CMAIDART DEVIATIOH

CORP?LASION

1332927
.792218

179.197

DEATERTHATIOH $\quad .527610$

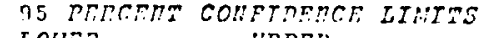

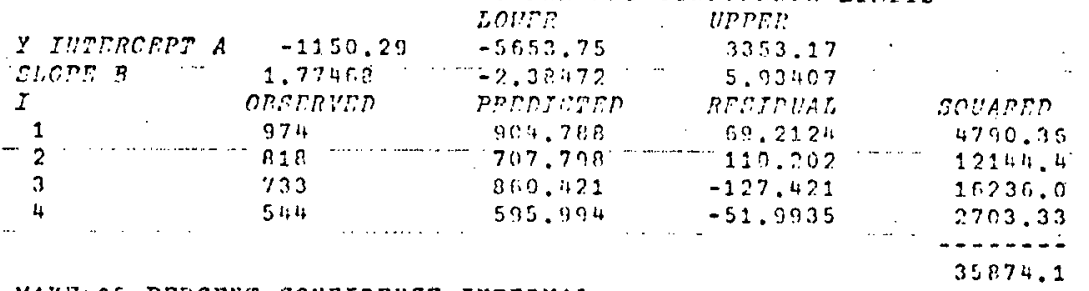

MAKF 95 RTRCEMT COHFJERHCF IMTENAL

FRERTCISTHE. TES O? 110

?YES

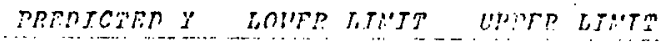

in:P:BX=

21002

627.938

192.45 .1

1053.41

TRY ANO"'ER. YRS OR

3110 


\section{APPENDIX F8}

PROJECTIONS TO 1981 FOR THIRTEEN DISTRICTS

BASED ON SEPTEMBER ENROLLMENTS

AND EIGHT YEAR TREND 
S U M M A R Y

SEPTEMBER ENROLLMENTS - PARISII OF HANOVER

1966/67 - 1973/74 and Projections

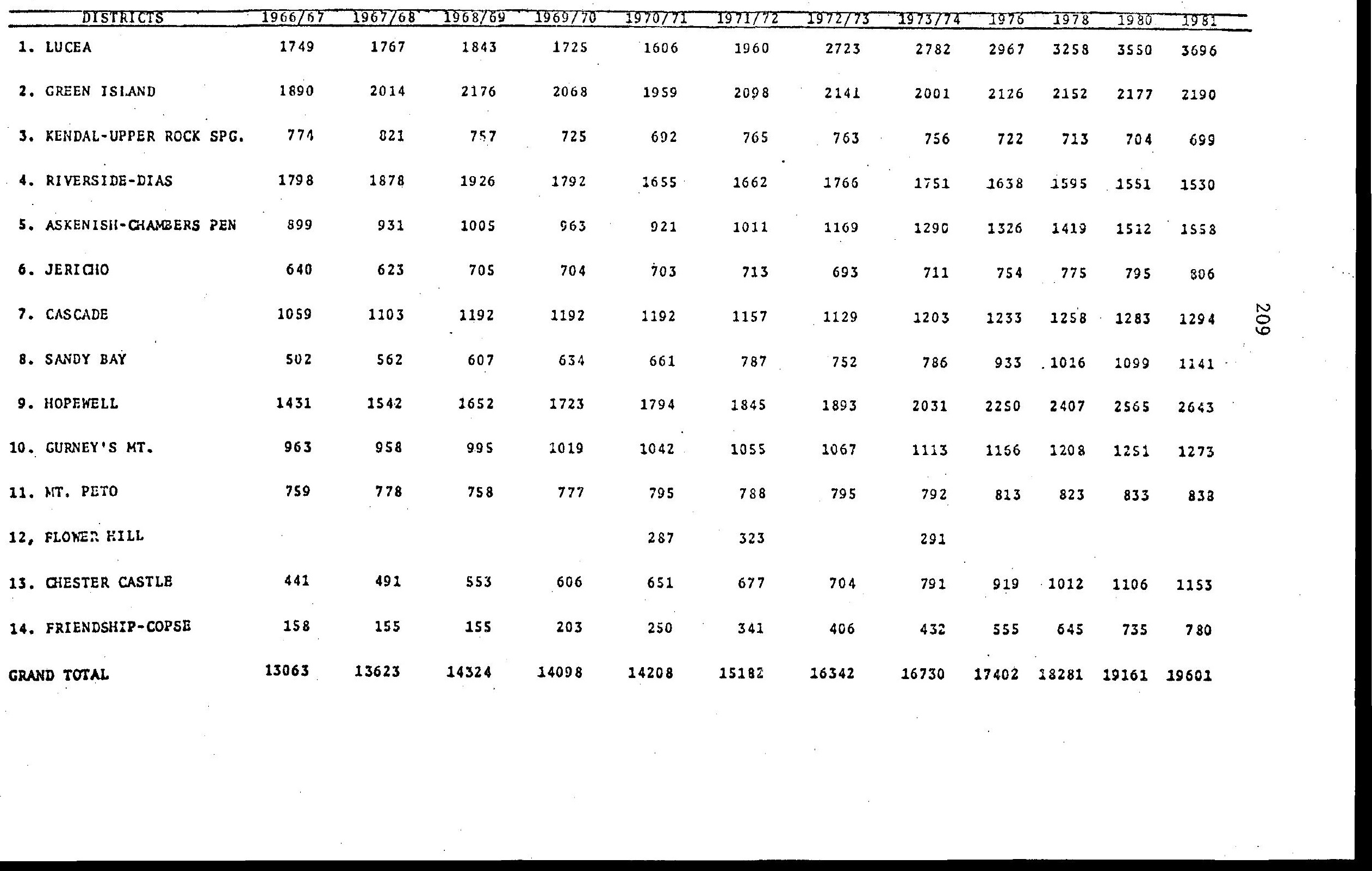


TIME ANALYSIS: PROJECTIONS: TO 1981 DISTRICT 非1, LUCEA

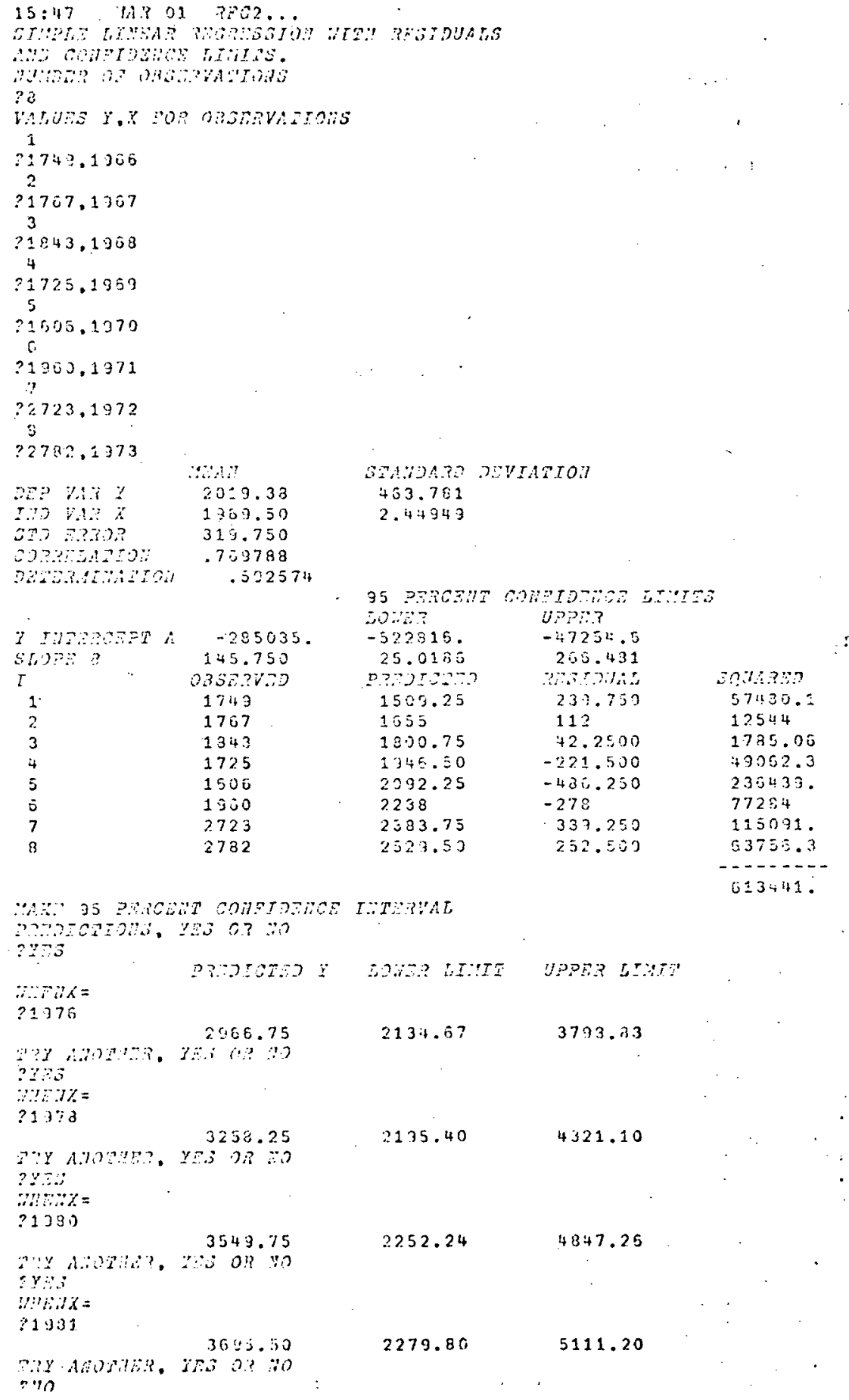

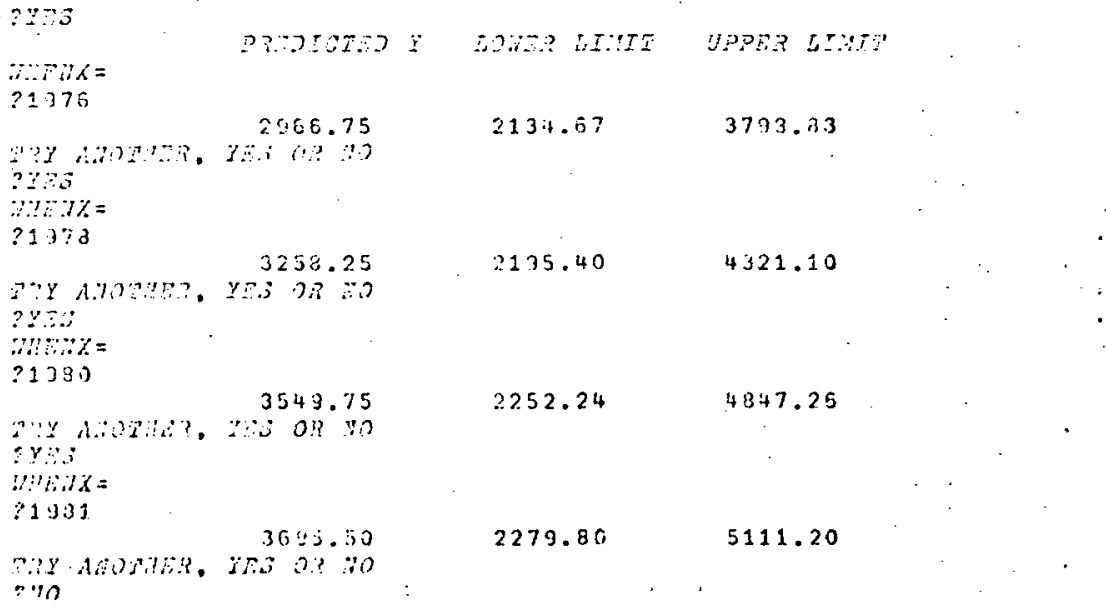


TIME ANALYSIS: PROJECTIONS TO 1981

DISTRTCT \&2, GREEN JSLAND

a..

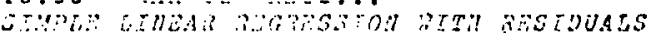

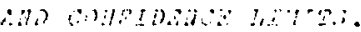

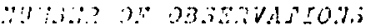

$\because$ is

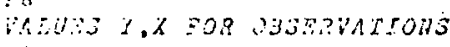

$? 13 \equiv 0.1306$

2

20014.1907

$22126,1.003$

i;

$2 \% 058,1903$

3

$?: 959.1370$

2200.1971

7

:?1:1,j:7?

22001.1973

ר)

rig $\forall$ :

$3202 \% ?$

...in:

2043.35

$1 ; 0.9 .50$

$37.5 \div 71$

.1202584

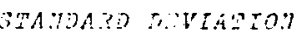

95.5272

2.44343

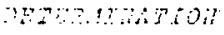

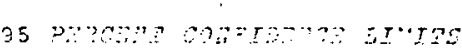

$2016 \%$
-19535.035

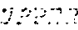

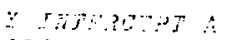

$-23020.3$

$-24.1434$

-

1933.33

$2930.7 \%$

3330

2011.50

49.5350

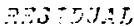

$-109.333$

2.44243

151.714

31. .2231

2037.01

$-30.7331$

35.5357

05.2375

2062.46

3075.12

62.2313
-85.3107

5? $211: \cdots$

1150.4 .7

5.25532

$23.31 \% .2$

90). 252

9233.45

1262.73

$433 \div .23$

$755 \div: \therefore 1$

57209.7

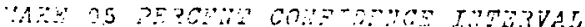

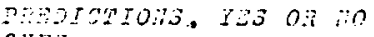

yㅣㄴ

$\because \because i x=$

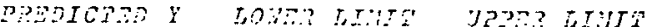

$2: 370$

$2125.10 \quad 1871.99 \quad 2360.20$

T)

$\therefore ., 8$

in: $i=$

$213 \% 2$

2151.55

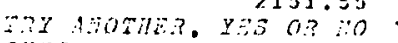

1825.27

2476.13

$? \because 2 \%$

21330

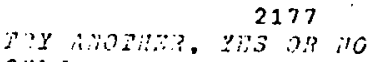

1780.70

2573.24

? 20.2

IIIAK $=$

?1931

2.187 .73

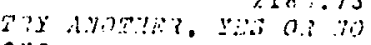

1757.39

2022.06

20 
TIME ANALYSIS: PROJECTIONS TO 1981

DISTRICT $\# 3$, KENDAL-UPPER ROCK SPRING

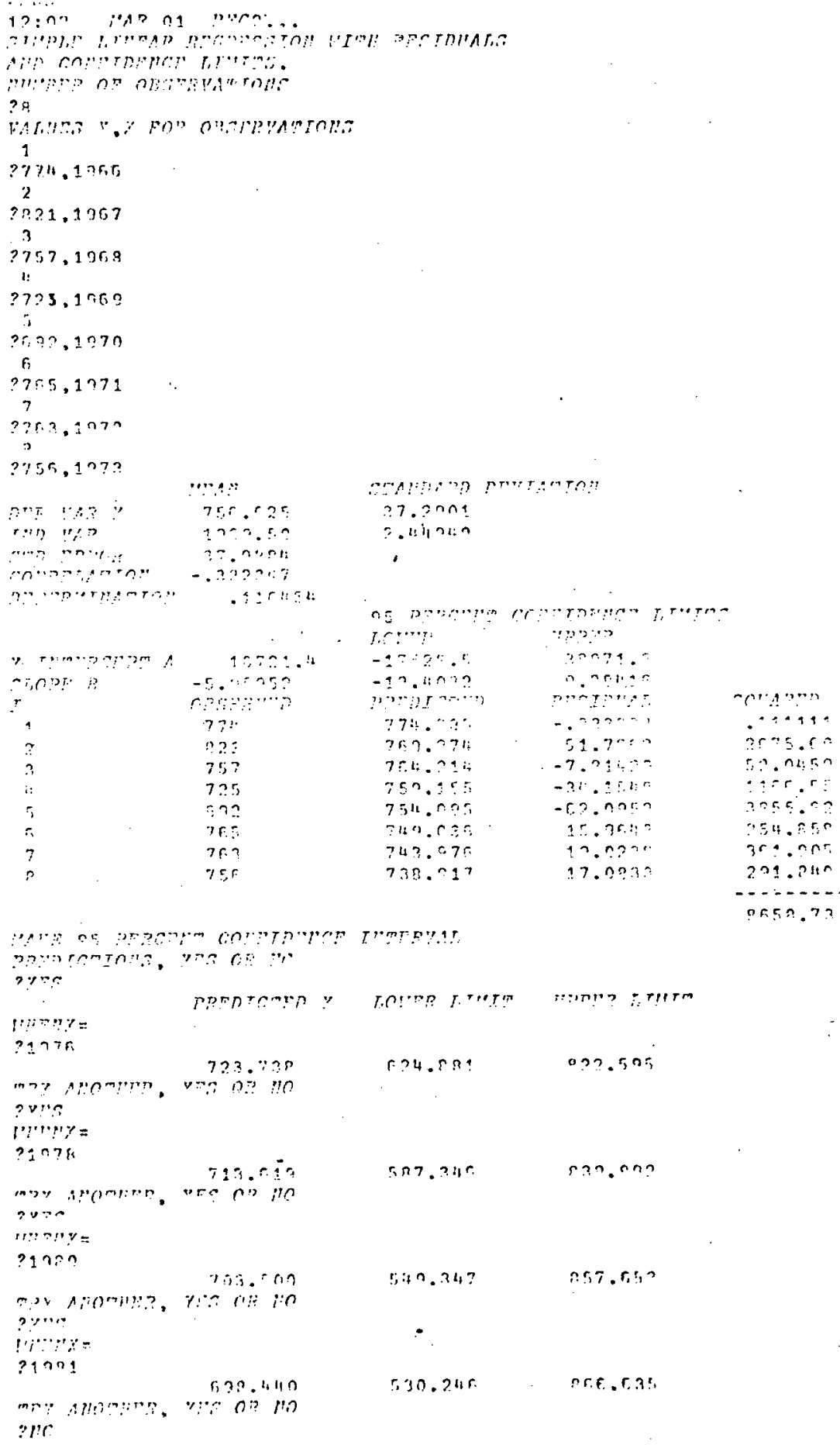


TIME ANALYSIS: PROJECTIONS TO 1981

DISTRICI \#4, RIVERSTDE-DIAS

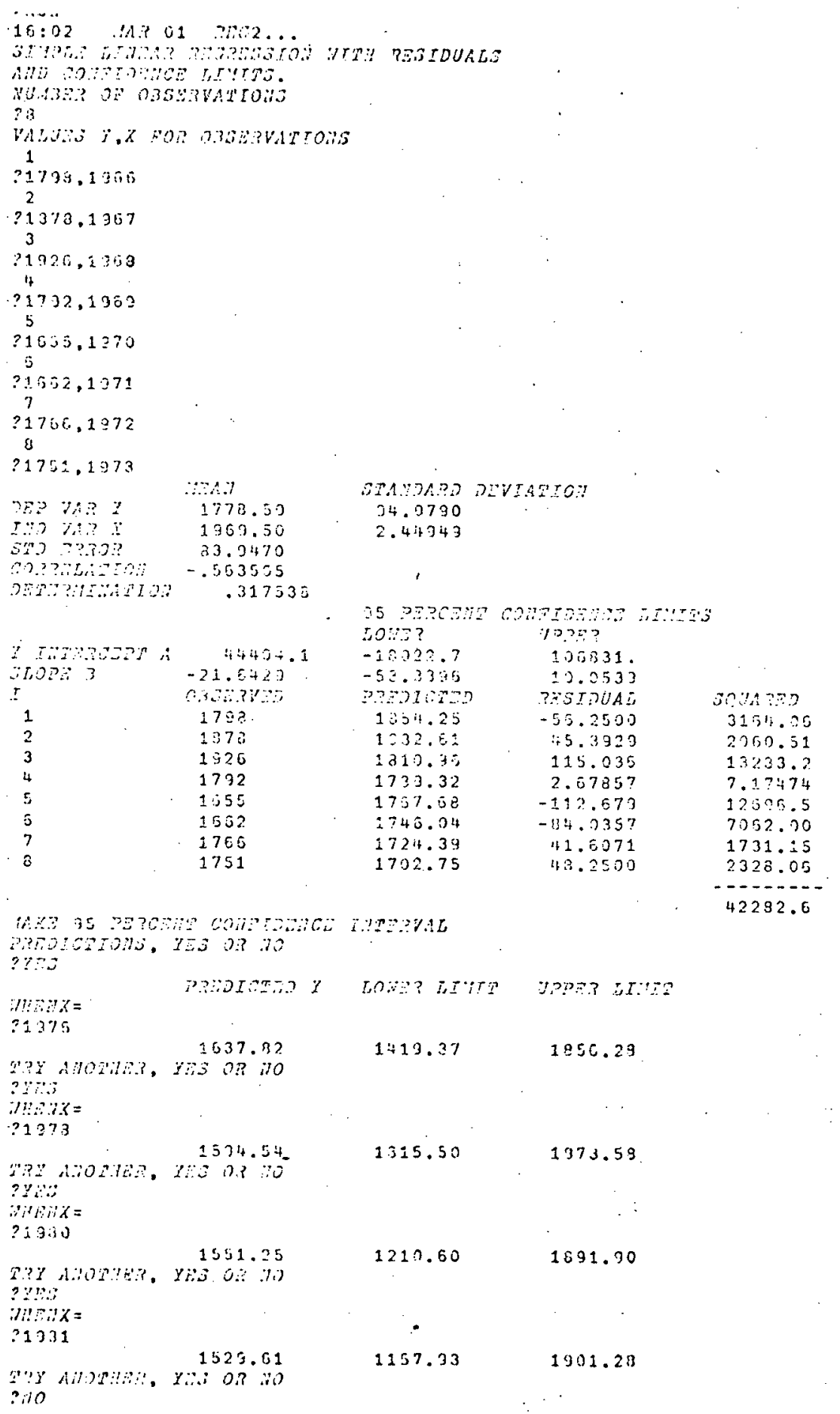


TIME ANALYSTS: PROJECTIONS TO 1981 DISTRICT \#5, ASKENISH

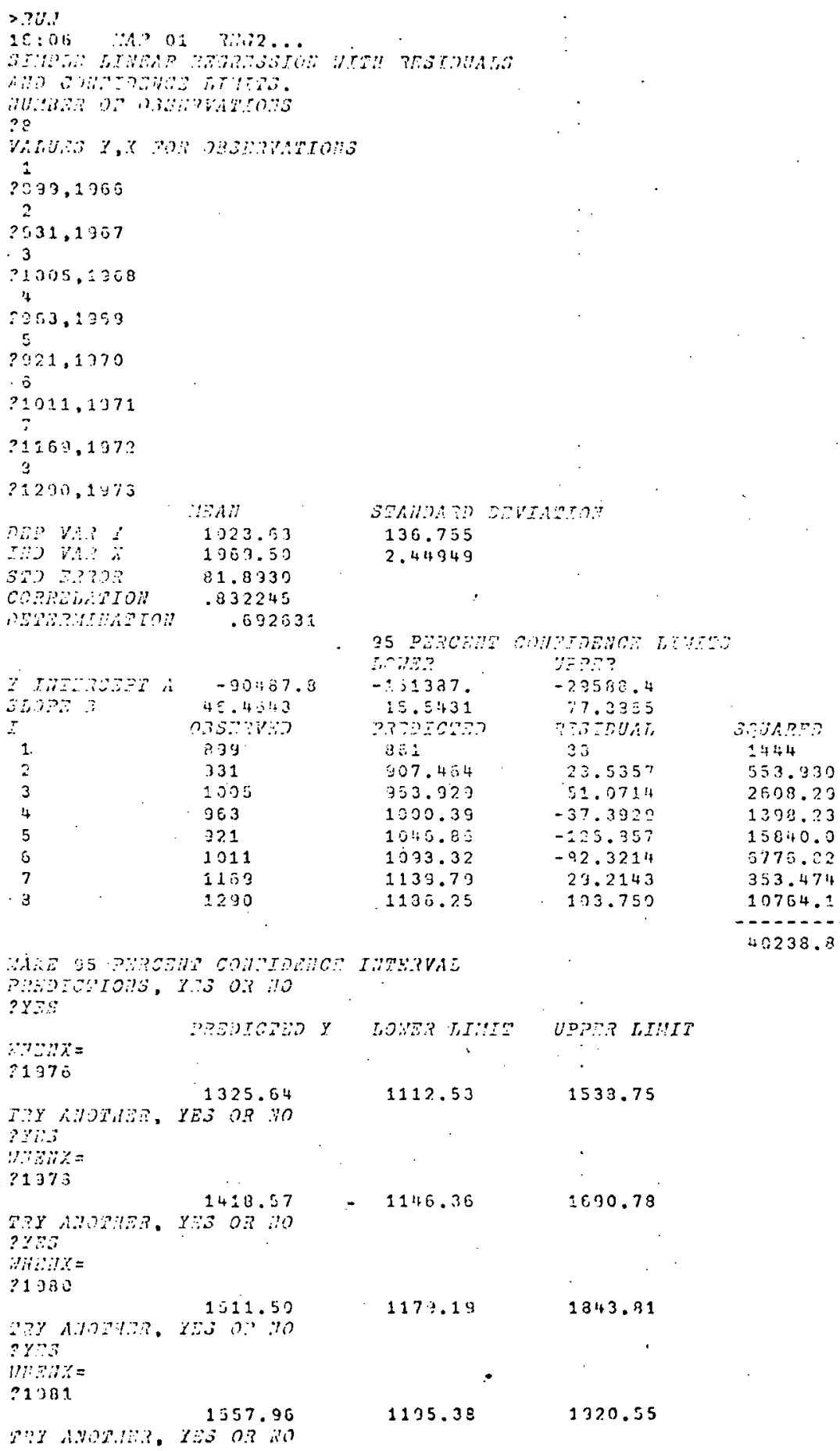

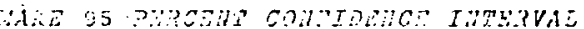

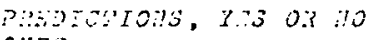

?Y>S

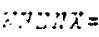

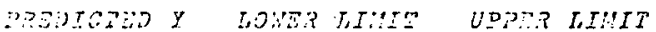

$21370^{\circ}$

$\begin{array}{lll}1325.64 & 1112.53 & 1533.75\end{array}$

T.Y AVIUAS, YEJ OR VO

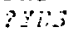

:3aik=

$? 1373$

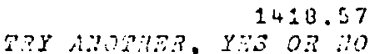

$3: 58$

HI:IIS=

$? 1980$

1511.50

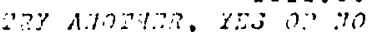

$3 \times 3$

1m: $x: x=$

?1281

1557.90

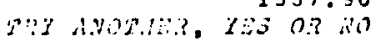

$117.19 \quad 1343.81$

$1135.38 \quad 1320.55$ 


\section{TIME ANALYSIS: PROJECTIONS TO 1.981 DESTRICT \#6, JERICHO}

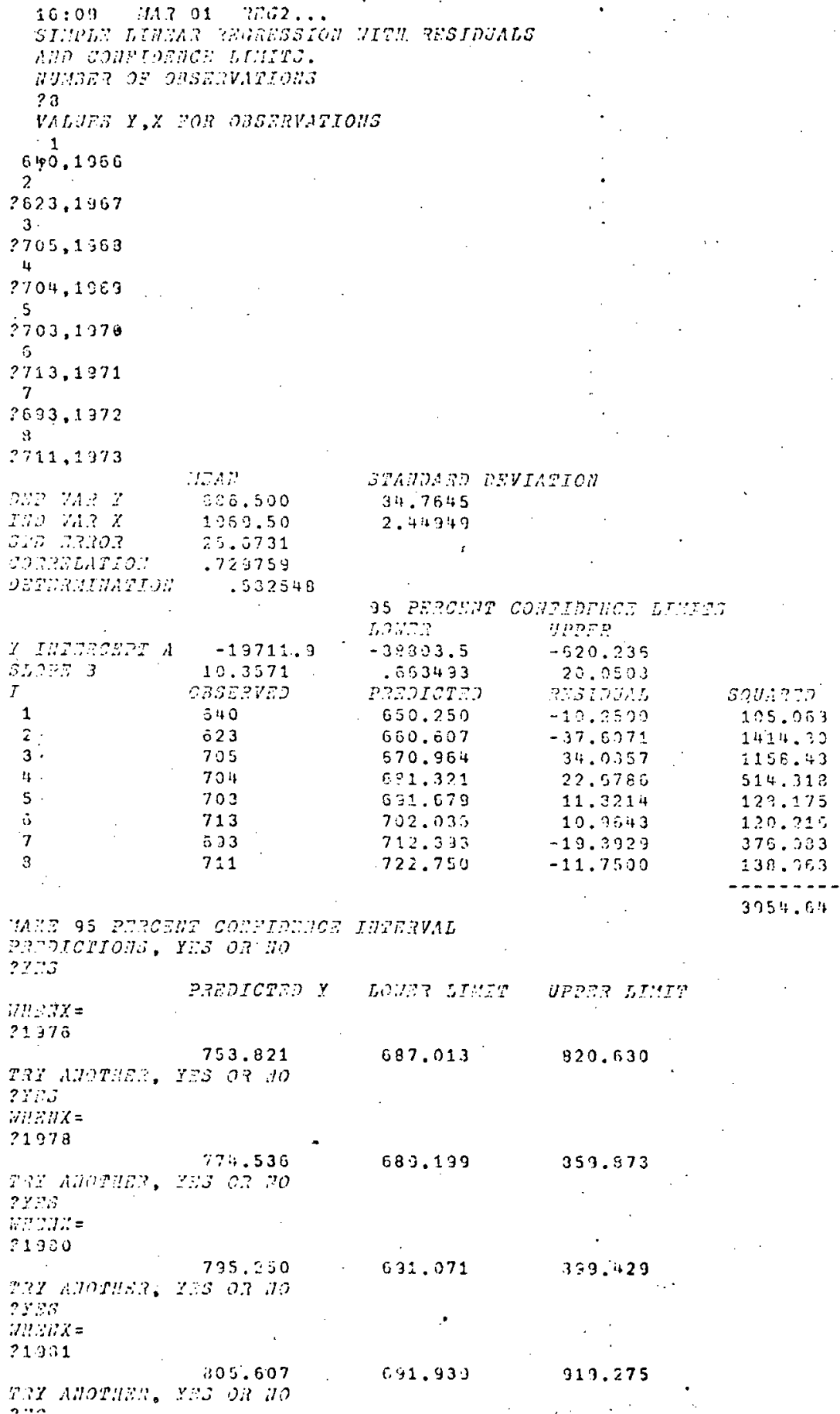


TIME ANALYSIS: PROJECTIONS TO 1981

DISTRICT \#7, CASCADE

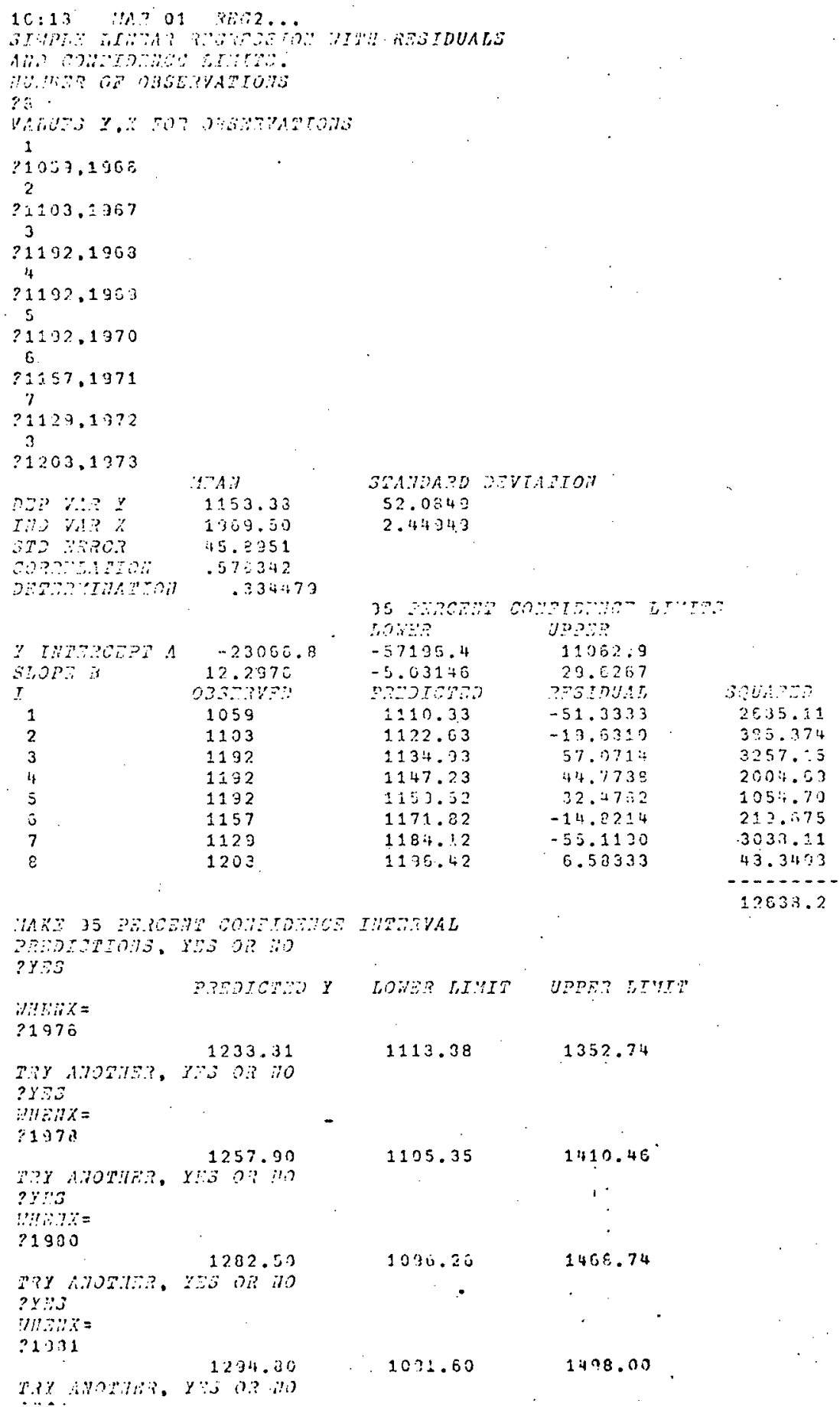

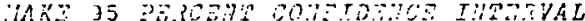

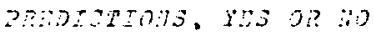

? Y 23

$\min \operatorname{tin} x=$

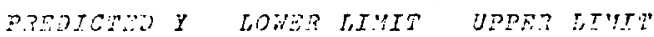

?1970̂

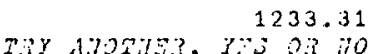

1113.38

$135 ? .74$

$39: 3$

mas $=$

$7197 \%$

1257.90

$1257 \cdot 90$
$30 \%: 110$

2Y:3

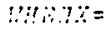

$? 1900$

1282.50

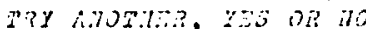

$3 y: 30$

III:AX $=$

$? 1331$

1234.30

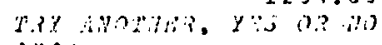

1105.35

$1410.4 \epsilon$

1.

1468.74

1030.20

$10: 2.60$

1498.00 
TIME ANALYSIS: PROJECTIONS TO 1981 DISTRICT \#8, SAYDY BAY

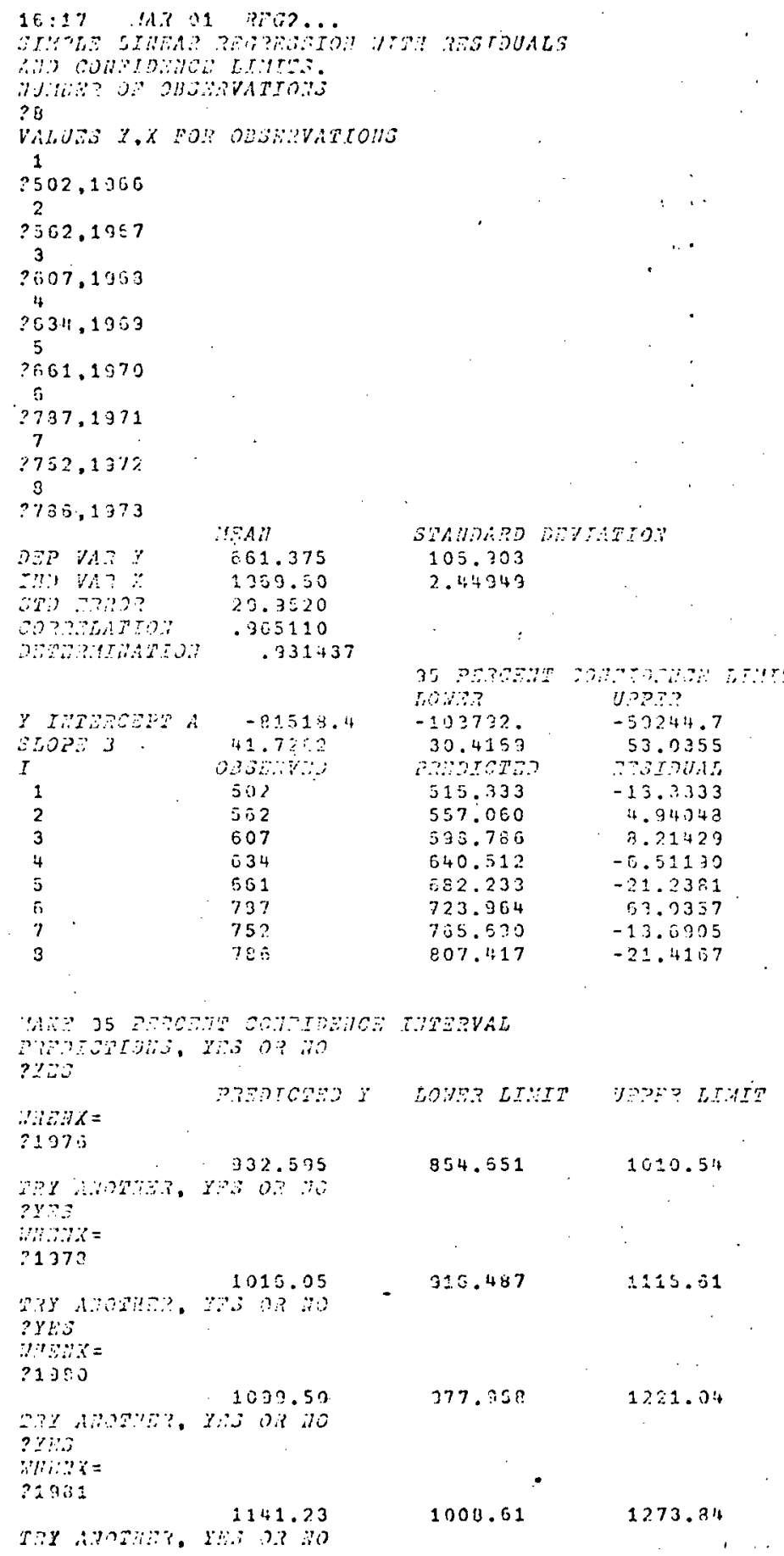


TIME ANALYSIS: PROJECTIONS TO 1981

DISTRICT \#9, HOPEWELL

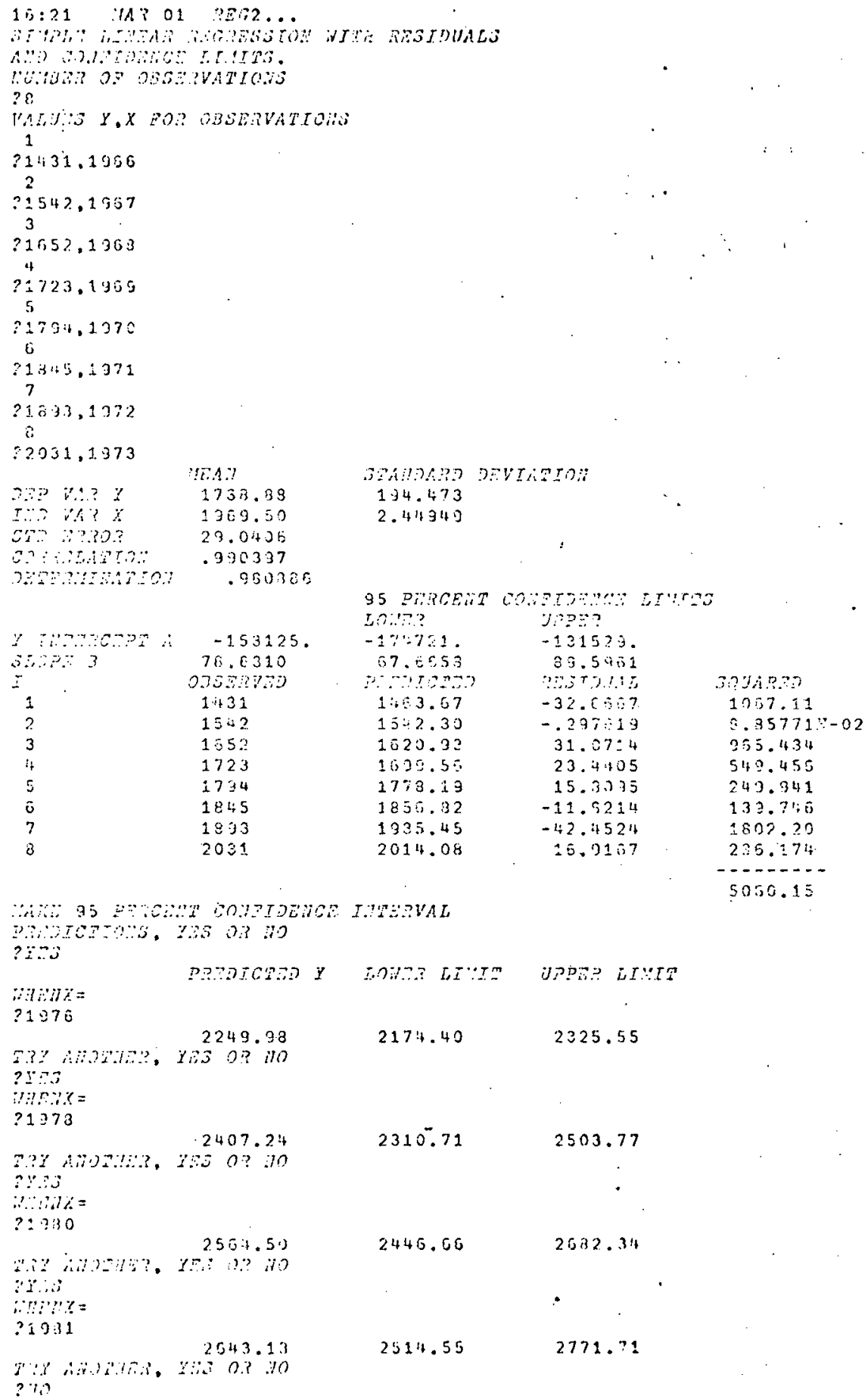

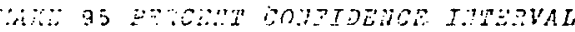

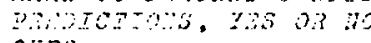


TIME ANALYSTS: PROJECTIONS TO 1981 DISTRICT \#10, GUERNEY'S MOUNT

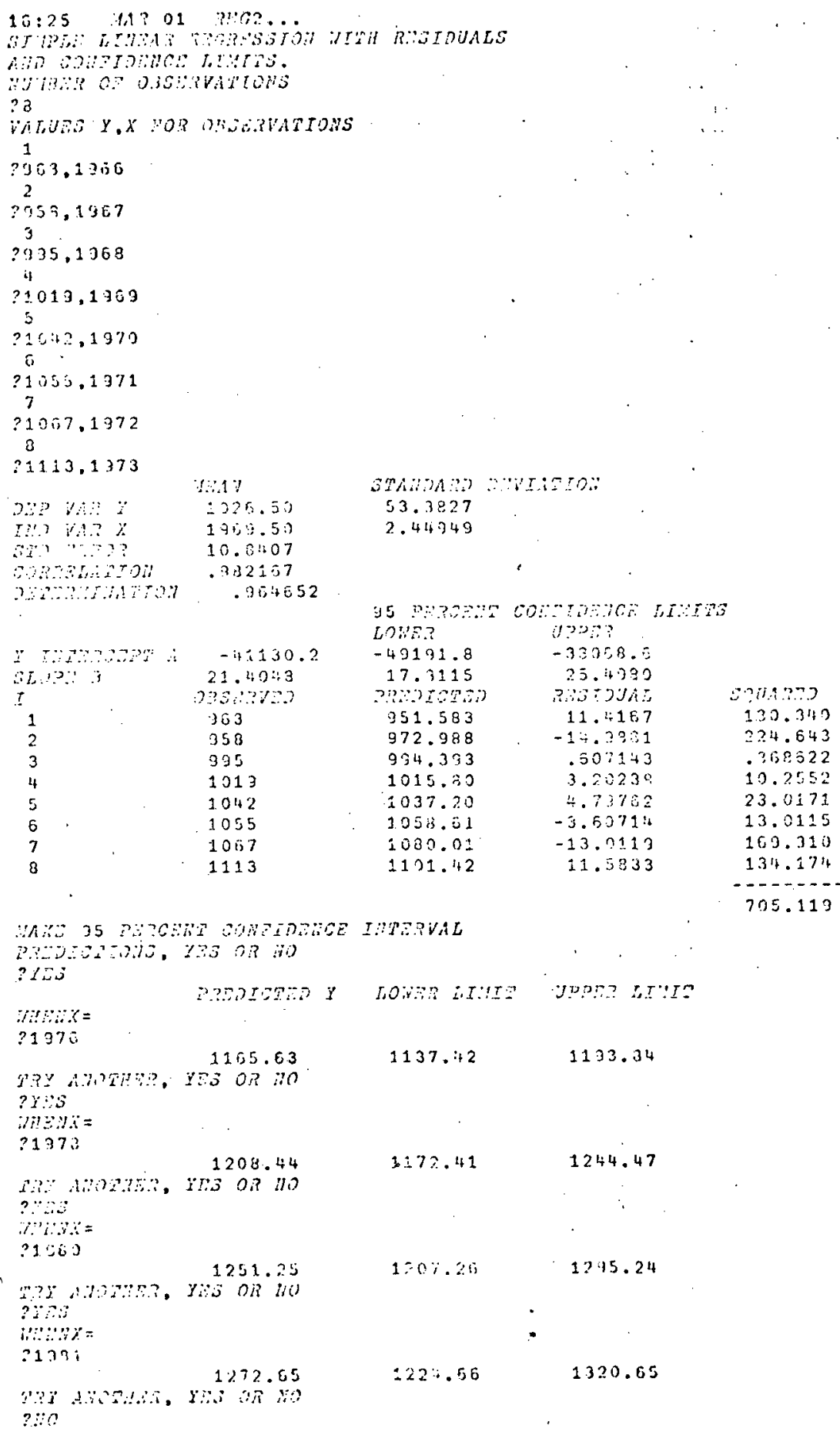


TIME ANALYSIS: PROJECTIONS TO 1981

DISTRICI \#II, MT. PETO

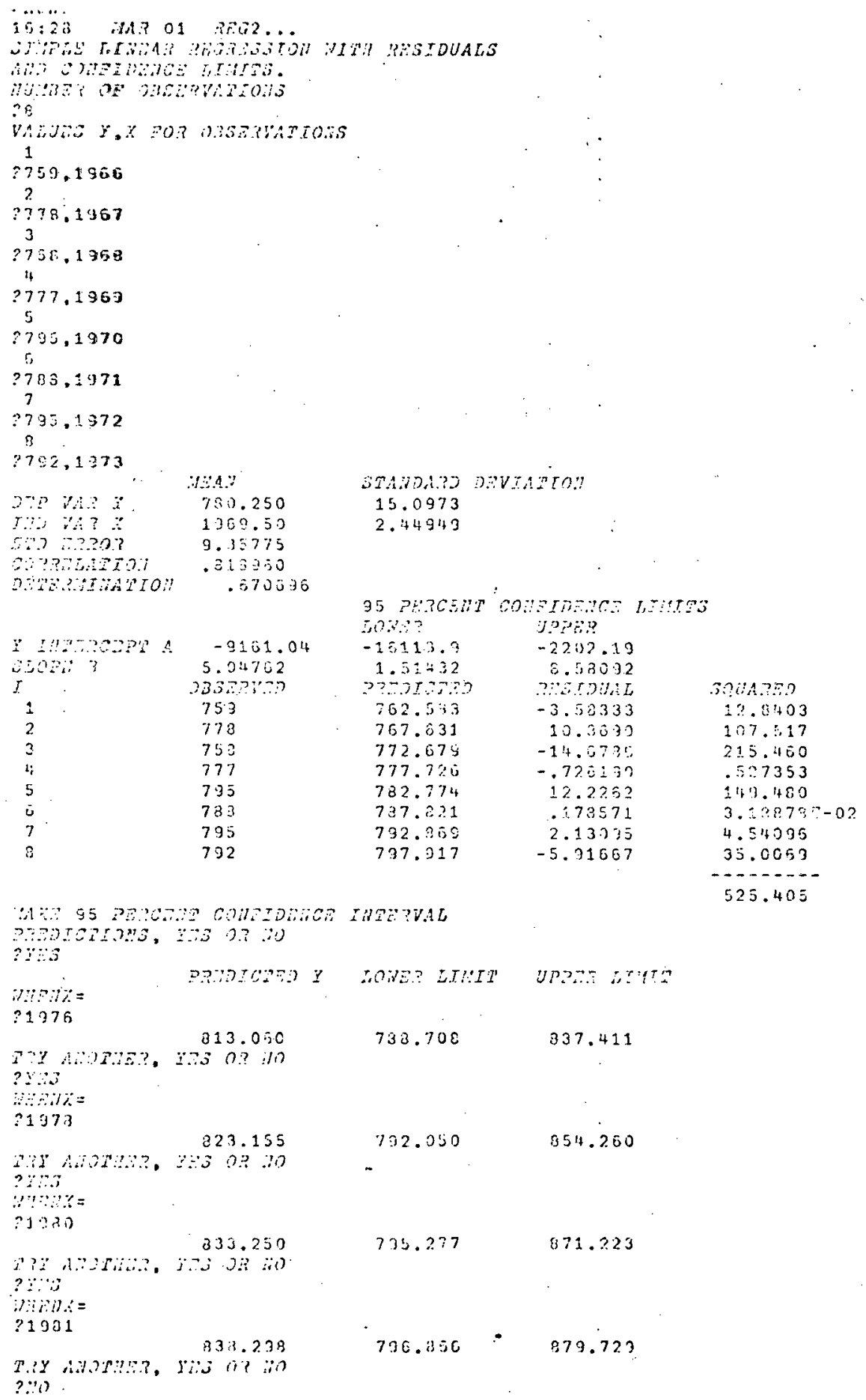


TIME ANALYSIS: PROJECTIONS TO 1981 DISTRICT \#13, CHESTER CASTLE

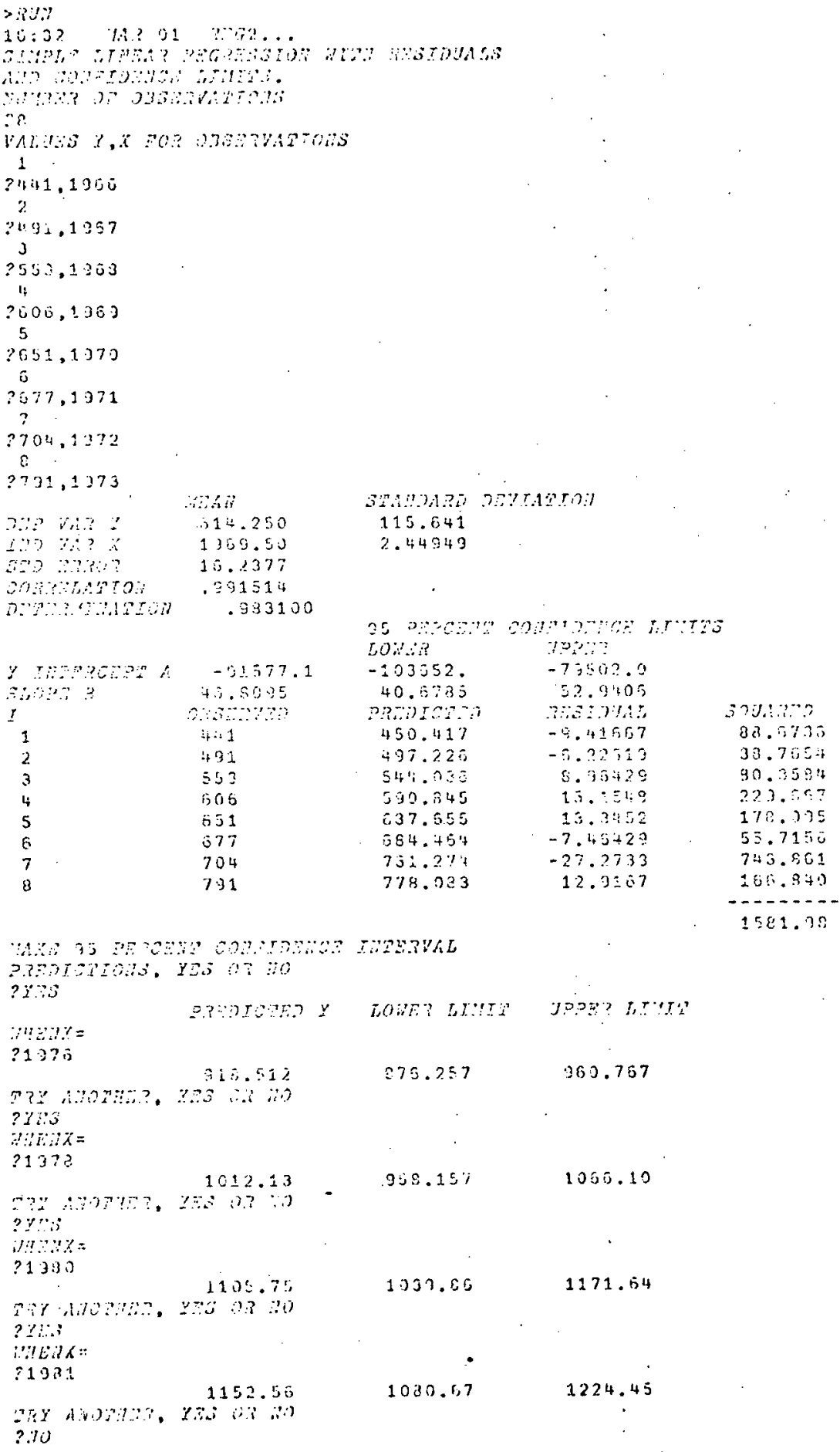


TIME ANALYSIS: PROJECTIONS TO 1981

DISTRICT \#14, FRIENDSHIP-COPSE

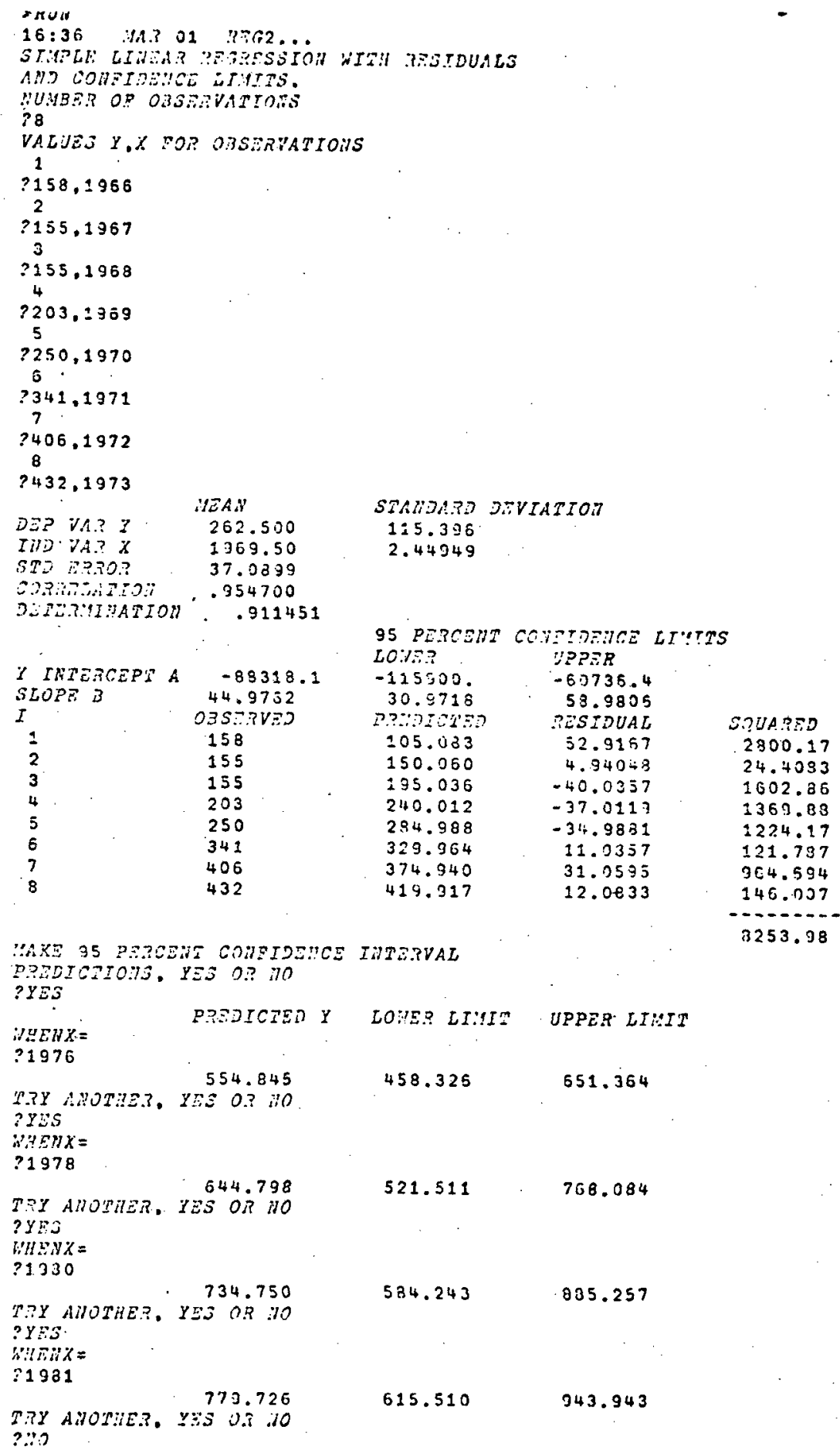

ZAKE 35 PERCEUT COHEIDENCE INTEZVAL

PRPDICITOHS. YES OP HO

? Y 53

$\because Y E W X=$

PREDICIED Y LOHEP LT:IT UPPEQ LIUIT

$? 1976$$$
\begin{array}{lll}
554.845 & 458.325 & 651.364
\end{array}
$$

TRY AROTZE, Y

?Yis

A.TIX $=$

$? 1978$

644.798

TRY AllOTILR. IES OR HO ?Y 15

IH:XX =

? 1330

TSY ALOTHE. Y 734.750 YYOS

औis $\operatorname{Rin}$

$\because 1931$

779.726

TRY AWOTHER. Y.YS OA .70 $?: 2$ 
APPENDIX G

CALCULATIONS---

THE COHORT SURVIVAT. METHOD 
THE COHORT SURVIVAL METHOD STRATUM 非 HOPEWELL

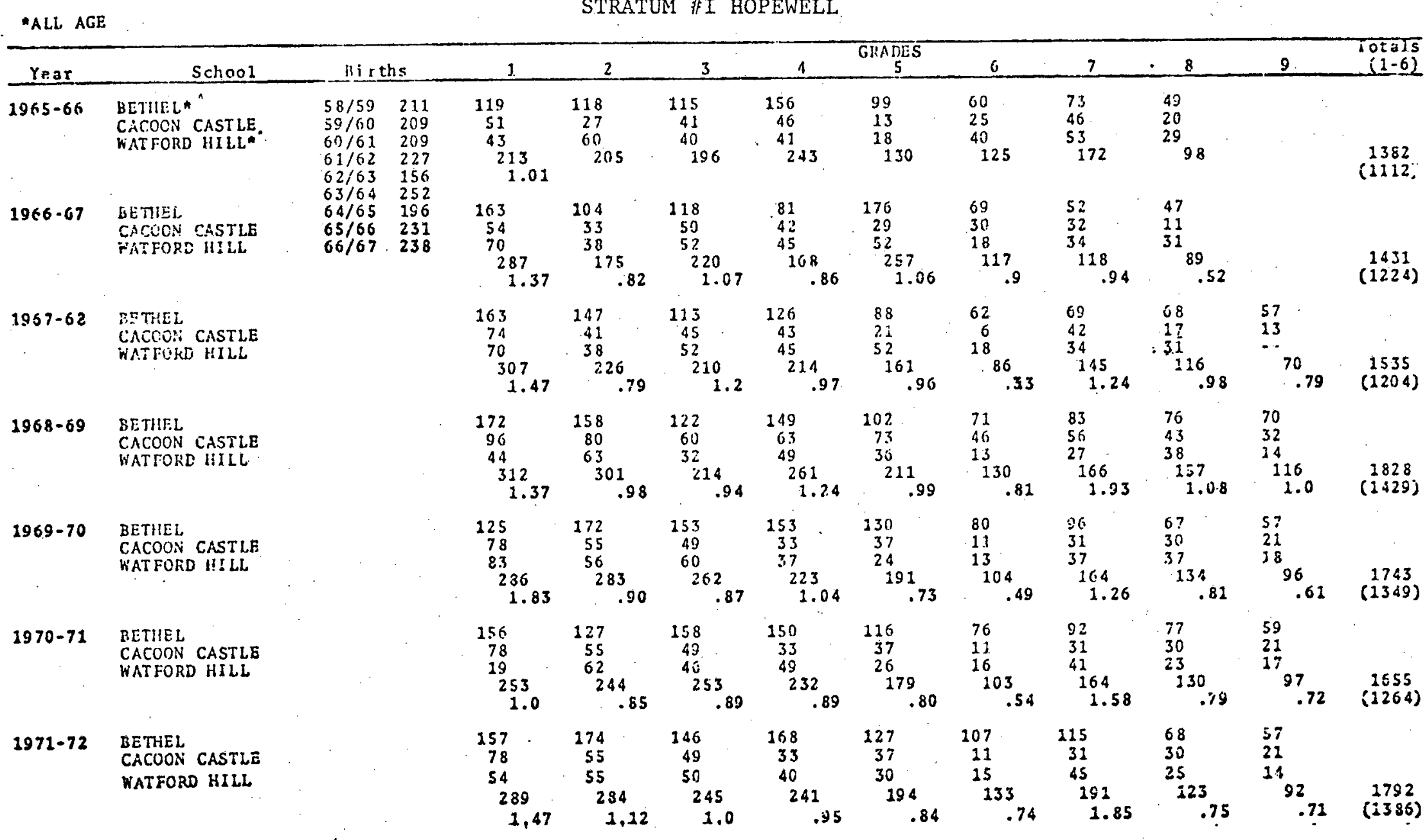


THE COHORT SURVIVAL METHOD

STRATUM 非 H HOPEWELL - cont'd

\begin{tabular}{|c|c|c|c|c|c|c|c|c|c|c|c|c|}
\hline Year & School & Births & 1 & 2 & 3 & 4 & GBADES & 0 & 7 & 8 & 9 & $\begin{array}{r}\text { Totals } \\
(1-6)\end{array}$ \\
\hline $1972-73$ & $\begin{array}{l}\text { BETHEL } \\
\text { CACOON CASTLE } \\
\text { WAT FORD HILL }\end{array}$ & & $\begin{array}{l}145 \\
78 \\
50 \\
273 \\
1.18\end{array}$ & $\begin{array}{r}159 \\
55 \\
65 \\
277 \\
\quad .96\end{array}$ & $\begin{array}{r}174 \\
49 \\
45 \\
268 \\
.94\end{array}$ & $\begin{array}{l}170 \\
33 \\
52 \\
255 \\
1.04\end{array}$ & $\begin{array}{r}149 \\
37 \\
25 \\
211 \\
.88\end{array}$ & $\begin{array}{l}37 \\
11 \\
15 \\
113 \\
\quad .58\end{array}$ & $\begin{array}{l}127 \\
31 \\
39 \\
197 \\
1.48\end{array}$ & $\begin{array}{l}89 \\
30 \\
19 \\
138 \\
\quad .72\end{array}$ & $\begin{array}{l}59 \\
21 \\
24 \\
104 \\
.84\end{array}$ & $\begin{array}{r}1836 \\
\text { (1397 }\end{array}$ \\
\hline $1973-74$ & $\begin{array}{l}\text { BETHEL } \\
\text { CACOON CASTLE } \\
\text { WATFORD HILL }\end{array}$ & & $\begin{array}{c}231 \\
78 \\
45 \\
354\end{array}$ & $\begin{array}{l}175 \\
55 \\
38 \\
268\end{array}$ & $\begin{array}{l}175 \\
49 \\
56 \\
280 \\
\end{array}$ & $\begin{array}{l}277 \\
33 \\
44 \\
254\end{array}$ & $\begin{array}{r}118 \\
37 \\
34 \\
189\end{array}$ & $\begin{array}{l}81 \\
11 \\
19 \\
111\end{array}$ & $\begin{array}{c}169 \\
31 \\
34 \\
234\end{array}$ & $\begin{array}{l}114 \\
30 \\
23 \\
167\end{array}$ & $\begin{array}{l}82 \\
21 \\
19 \\
122\end{array}$ & $\begin{array}{c}1979 \\
(1456)\end{array}$ \\
\hline $\begin{array}{l}\text { Average } \\
\text { Projecti } \\
\text { Average } \\
\text { Projecti }\end{array}$ & $\begin{array}{l}\text { atio to } 1970 / 71 \\
\text { ns to } 1973 / 74 \\
\text { atio to } 1972 / 73 \\
\text { ns to } 1973 / 74\end{array}$ & & $\begin{array}{l}1.34 \\
287^{3} \\
287^{34}\end{array}$ & $\begin{array}{l}250^{87} \\
264^{.92}\end{array}$ & $\begin{array}{l}24 \dot{7}^{.99} \\
26 i^{99}\end{array}$ & $\begin{array}{c}1.0 \\
247^{\circ} \\
1.0 \\
26 i\end{array}$ & $22 \dot{5}^{.91}$ & $137^{.61}$ & $\begin{array}{l}1.39 \\
190^{39} \\
1.5^{47}\end{array}$ & $17 \dot{8}^{.84}$ & $\begin{array}{l}12 \dot{5}^{78} \\
13 \dot{6}^{78}\end{array}$ & $\begin{array}{l}2868 \\
1976\end{array}$ \\
\hline
\end{tabular}


THE COHORT SURVIVAL METHOD

STRATUM 非II SANDY BAY

*Interpolated by computer

\begin{tabular}{|c|c|c|c|c|c|c|c|c|c|c|c|c|c|}
\hline Year & School & Birt & & 1 & 2 & 3 & $4^{\cdots}$ & $\begin{array}{c}\text { GPADES } \\
5 \\
\end{array}$ & 6 & $\begin{array}{r}7 \\
\end{array}$ & 8 & 2 & $\begin{array}{r}\text { Totals } \\
(1-6) \\
\end{array}$ \\
\hline $1965-66$ & SANDY BAY & $\begin{array}{l}58 / 59 \\
59 / 60 \\
60 / 61 \\
61 / 62\end{array}$ & $\begin{array}{r}108 \\
106 \\
113 \\
94\end{array}$ & $\begin{array}{l}84 \\
.77\end{array}$ & 69 & 134 & 57 & 28 & 47 & 62 & 33 & & $\begin{array}{c}514 \\
(419)\end{array}$ \\
\hline $1966-67$ & SANDY BAY & $\begin{array}{l}62 / 63 \\
63 / 64 \\
64 / 65 \\
65 / 66\end{array}$ & $\begin{array}{r}91 \\
89 \\
117 \\
122\end{array}$ & $\begin{array}{l}64 \\
.60\end{array}$ & $\begin{array}{l}87 \\
1.04\end{array}$ & $\begin{array}{l}86 \\
1.25\end{array}$ & $\begin{array}{l}79 \\
.59\end{array}$ & $\begin{array}{l}66 \\
1.16\end{array}$ & $\begin{array}{l}52 \\
1.86\end{array}$ & $\begin{array}{l}31 \\
\quad .66\end{array}$ & $\begin{array}{l}34 \\
.55\end{array}$ & & $\begin{array}{c}499 \\
(434)\end{array}$ \\
\hline $1967-68$ & SANDY BAY & $66 / 67$ & 104 & $\begin{array}{r}108 \\
.96\end{array}$ & $\begin{array}{l}71 \\
1.11\end{array}$ & $\begin{array}{l}89 \\
1.02\end{array}$ & $\stackrel{65}{.76}$ & $\begin{array}{l}86 \\
1.09\end{array}$ & $\begin{array}{l}61 * \\
.92\end{array}$ & $\begin{array}{l}60 \\
1.15\end{array}$ & $\begin{array}{l}47 \\
1.52\end{array}$ & $\begin{array}{l}30 \\
.88\end{array}$ & $\begin{array}{c}556 \\
(419)\end{array}$ \\
\hline $1968 \cdot 69$ & SANDY BAY & & & $\begin{array}{l}126 \\
1.34\end{array}$ & $\stackrel{99}{.92}$ & $\begin{array}{l}77 \\
1.08\end{array}$ & $\begin{array}{l}118 \\
1.33\end{array}$ & 51.78 & $\begin{array}{l}41 \\
. \quad .48\end{array}$ & $\begin{array}{l}63 \\
1.03\end{array}$ & $\begin{array}{l}52 \\
.86\end{array}$ & $\begin{array}{l}30 \\
.64\end{array}$ & $\begin{array}{c}657 \\
(512)\end{array}$ \\
\hline $1969-70$ & SANDY BAYY & & & $\begin{array}{l}122 \\
1.34\end{array}$ & $\begin{array}{l}105 \\
.83\end{array}$ & $\stackrel{93}{.94}$ & $\begin{array}{l}73 \\
.95\end{array}$ & ${ }^{72} .61$ & $\begin{array}{l}33 \\
.65\end{array}$ & $\begin{array}{l}83 \\
2.02\end{array}$ & $\begin{array}{l}36 \\
.57\end{array}$ & $\begin{array}{l}58 \\
.67\end{array}$ & $\begin{array}{c}675 \\
(498)\end{array}$ \\
\hline $1970-71$ & SANDY BAY & & . & $\begin{array}{l}102 \\
1.15\end{array}$ & $\begin{array}{l}142 \\
1.16\end{array}$ & $\begin{array}{l}111 \\
1.06\end{array}$ & $\stackrel{75}{.81}$ & $\stackrel{45}{.62}$ & $\stackrel{36}{.50}$ & $\begin{array}{l}66 \\
2 .\end{array}$ & ${ }^{47} .57$ & $\stackrel{27}{.75}$ & $\begin{array}{c}65 i \\
(512)\end{array}$ \\
\hline 1971-72 & SANDY BAY & & & $\begin{array}{l}130 \\
1.11\end{array}$ & $\begin{array}{l}97 \\
.95\end{array}$ & $\begin{array}{l}13.3 \\
.94\end{array}$ & $\begin{array}{r}101 \\
.91\end{array}$ & $\begin{array}{l}73 \\
.9 .7\end{array}$ & $\begin{array}{l}29 \\
.64\end{array}$ & $\begin{array}{l}70 \\
1.94\end{array}$ & $\begin{array}{l}31 \\
.47\end{array}$ & $\begin{array}{l}32 \\
.68\end{array}$ & $\begin{array}{c}696 \\
(563)\end{array}$ \\
\hline $1972-73$ & SANDY BAY & & & $\begin{array}{r}120 \\
.98\end{array}$ & $\begin{array}{l}130 \\
1.05\end{array}$ & 96.99 & $\begin{array}{r}122 \\
.92\end{array}$ & 82 & $\begin{array}{l}30 \\
.41\end{array}$ & $\begin{array}{l}94 \\
3.24\end{array}$ & $\begin{array}{l}38 \\
.54\end{array}$ & $\begin{array}{l}34 \\
1.20\end{array}$ & $\begin{array}{c}752 \\
(586)\end{array}$ \\
\hline 1973-74 & SANDY BAY & & & 123 & 123 & 128 & 83 & 95 & 49 & 114 & 40 & 31 & $\begin{array}{c}786 \\
(601)\end{array}$ \\
\hline $\begin{array}{l}\text { Average } \\
\text { Projecti } \\
\text { Average } \\
\text { Project }\end{array}$ & $\begin{array}{l}\text { os to } 1970 / 71 \\
\text { to } 1973 / 74 \\
\text { os to } 1972 / 73 \\
\text { to } 1973 / 74\end{array}$ & & & $\begin{array}{l}1.03 \\
108 \\
1.03 \\
108\end{array}$ & $\begin{array}{l}1.01 \\
109 \\
1.0 \\
108\end{array}$ & $\begin{array}{l}1.07 \\
117 \\
1.04 \\
112\end{array}$ & $\begin{array}{c}104^{89} \\
10 i^{90}\end{array}$ & $\begin{array}{l}85 \\
88 \\
87^{86}\end{array}$ & $\frac{.88}{6 \dot{7}^{78}}$ & $\begin{array}{l}1.37 \\
105 \\
2.72 \\
117\end{array}$ & $\begin{array}{l}8 \dot{5}^{81} \\
8 \dot{5}^{73}\end{array}$ & $63^{.79}$ & $\begin{array}{l}856 \\
853\end{array}$ \\
\hline
\end{tabular}


THE COHORT SURVIVAL METHOD

STRATUM 非III KENDAL-UPPER ROCK SPRING

interpolated by Computer

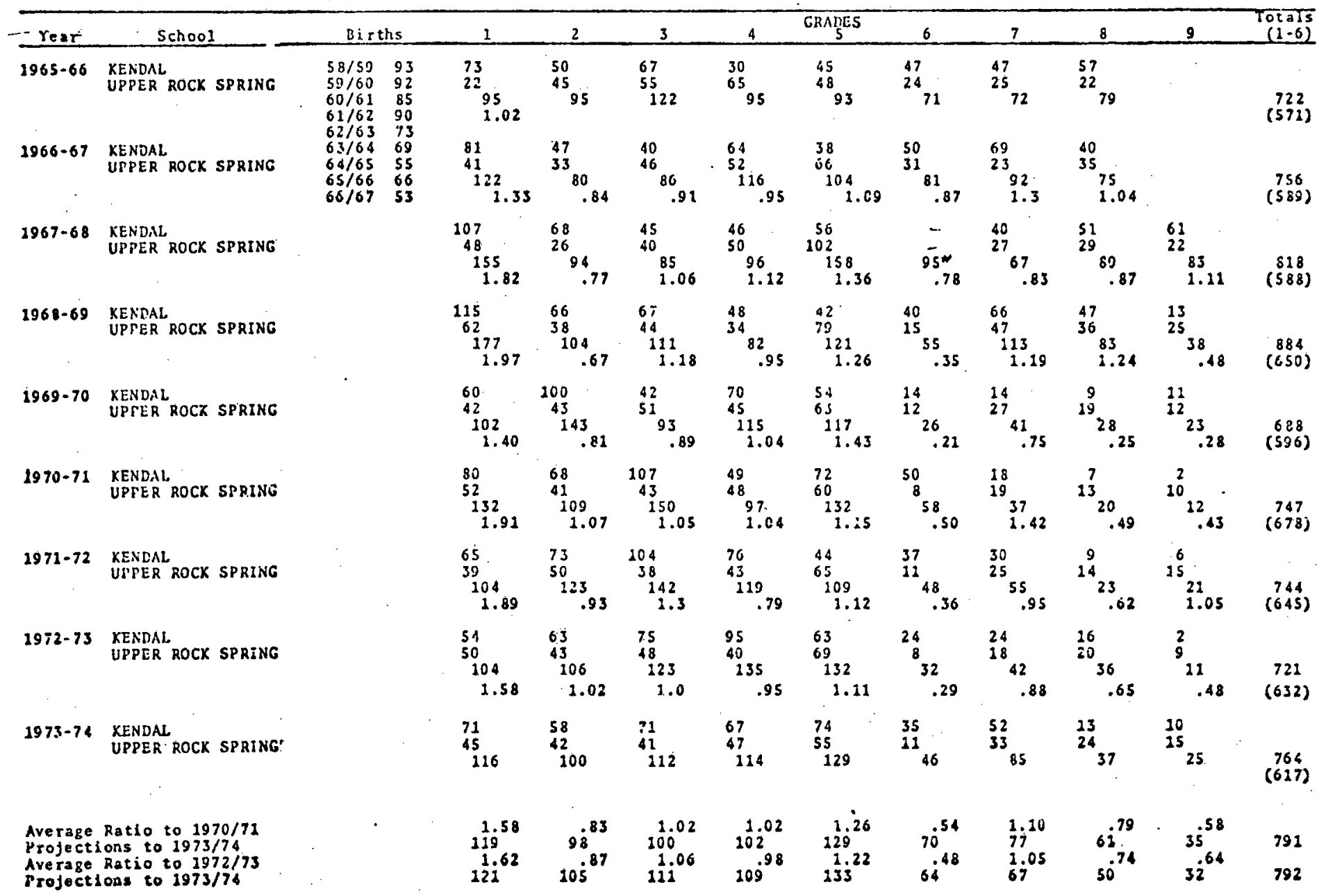


THE COHORT SURVIVAL METHOD

STRATUM \#IV JERICHO

An - Al1 Age

\begin{tabular}{|c|c|c|c|c|c|c|c|c|c|c|c|c|c|c|}
\hline Yeat & Scho & & Birt & & 1 & 2 & 3 & 4 & $\begin{array}{c}\text { GrANES } \\
5 \\
\end{array}$ & 6 & 7 & 8 & 9 & $\begin{array}{r}\text { Tota } 15 \\
(1-6) \\
\end{array}$ \\
\hline $1965-66$ & $\begin{array}{l}\text { CLARENIONT } \\
\text { JERICHOO }\end{array}$ & $A A^{*}$ & $\begin{array}{l}58 / 59 \\
59 / 60 \\
60 / 61 \\
61 / 62 \\
62 / 63\end{array}$ & $\begin{array}{r}110 \\
108 \\
105 \\
107 \\
91\end{array}$ & $\begin{array}{l}39 \\
69 \\
108 \\
\quad .98\end{array}$ & $\begin{array}{l}29 \\
64 \\
\quad 93\end{array}$ & $\begin{array}{l}26 \\
64 \\
90\end{array}$ & $\begin{array}{l}16 \\
80 \\
96\end{array}$ & $\begin{array}{l}29 \\
38 \\
67\end{array}$ & ${ }_{64}^{21}$ & $\begin{array}{l}25 \\
22 \\
47\end{array}$ & $\begin{array}{l}34 \\
25 \\
59\end{array}$ & & $\begin{array}{l}624 \\
(518)\end{array}$ \\
\hline 1966.67 & $\begin{array}{l}\text { CLARENIONT } \\
\text { JERI CHO }\end{array}$ & $M$ & $\begin{array}{l}63 / 64 \\
64 / 65 \\
65 / 66 \\
66 / 67\end{array}$ & $\begin{array}{l}77 \\
73 \\
86 \\
78\end{array}$ & $\begin{array}{l}30 \\
95 \\
125 \\
1.16\end{array}$ & $\begin{array}{l}30 \\
64 \\
94 \\
.87\end{array}$ & $\begin{array}{l}27 \\
47 \\
\quad 74 \\
\quad .80\end{array}$ & $\begin{array}{l}34 \\
52 \\
\quad 86 \\
\quad .96\end{array}$ & $\begin{array}{l}30 \\
52 \\
\quad 82 \\
\quad .85\end{array}$ & $\begin{array}{l}22 \\
68 \\
90 \\
1.34\end{array}$ & $\begin{array}{l}25 \\
32 \\
\quad 57 \\
\quad .89\end{array}$ & $\begin{array}{l}22 \\
27 \\
\quad 49 \\
1.04\end{array}$ & & $\begin{array}{r}657 \\
(551)\end{array}$ \\
\hline $1967-68$ & $\begin{array}{l}\text { CLAREMONT } \\
\text { JERI DIO }\end{array}$ & AA & & & $\begin{array}{l}24 \\
69 \\
93 \\
\quad .89\end{array}$ & $\begin{array}{l}23 \\
60 \\
83 \\
.66\end{array}$ & $\begin{array}{l}24 \\
68 \\
92 \\
\quad .98\end{array}$ & $\begin{array}{l}28 \\
55 \\
83 \\
1.28\end{array}$ & $\begin{array}{l}15 \\
18 \\
\quad 33 \\
\quad .38\end{array}$ & $\begin{array}{l}8 \\
64^{*} \\
.78\end{array}$ & $\begin{array}{l}19 \\
44 \\
\quad 63 \\
\quad .70\end{array}$ & $\begin{array}{l}20 \\
40 \\
60 \\
1.05\end{array}$ & $\begin{array}{l}21 \\
33 \\
54 \\
1.1\end{array}$ & $\begin{array}{c}539 \\
(463)\end{array}$ \\
\hline $1968-69$ & $\begin{array}{l}\text { CLARLMONT } \\
\text { JERIOIO }\end{array}$ & $M$ & & & $\begin{array}{l}55 \\
95 \\
150 \\
1.40\end{array}$ & $\begin{array}{l}22 \\
74 \\
96 \\
1.03\end{array}$ & $\begin{array}{l}21 \\
50 \\
\quad 71 \\
\quad .86\end{array}$ & $\begin{array}{l}25 \\
79 \\
104 \\
1.13\end{array}$ & $\begin{array}{l}20 \\
39 \\
\quad 59 \\
\quad .71\end{array}$ & 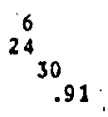 & $\begin{array}{l}22 \\
53 \\
\quad 75 \\
2.17\end{array}$ & $\begin{array}{l}13 \\
34 \\
\quad 47 \\
\end{array}$ & $\begin{array}{l}22 \\
28 \\
\quad 49 \\
\quad .8\end{array}$ & $\begin{array}{l}681 \\
(510)\end{array}$ \\
\hline $1969-70$ & $\begin{array}{l}\text { ClAAREMONT } \\
\text { JERI CIO }\end{array}$ & $M$ & & . & $\begin{array}{l}41 \\
69 \\
110 \\
1.21\end{array}$ & $\begin{array}{l}37 \\
76 \\
113 \\
.75\end{array}$ & $\begin{array}{l}25 \\
62 \\
\quad 87 \\
\quad .91\end{array}$ & $\begin{array}{l}3 . \\
76 \\
\quad 79 \\
1.11\end{array}$ & $\begin{array}{l}33 \\
46 \\
\quad 79 \\
.76\end{array}$ & ${ }_{30}^{21.51}$ & $\begin{array}{l}14 \\
82 \\
96 \\
3.1 ?\end{array}$ & $\begin{array}{l}20 \\
37 \\
57 \\
\quad .76\end{array}$ & $\begin{array}{l}13 \\
29 \\
42 \\
.89\end{array}$ & $\begin{array}{c}693 \\
(498)\end{array}$ \\
\hline $1979-71$ & $\begin{array}{l}\text { CLAREMONT } \\
\text { JERI CHO }\end{array}$ & $\mathbf{M A}$ & & & $\begin{array}{l}33 \\
69 \\
102 \\
1.32\end{array}$ & $\begin{array}{l}39 \\
78 \\
117 \\
1.06\end{array}$ & $\begin{array}{l}42 \\
68 \\
110 \\
.97\end{array}$ & $\begin{array}{l}23 \\
57 \\
{ }^{80} \\
\quad .92\end{array}$ & $\begin{array}{l}33 \\
59 \\
92 \\
1.26\end{array}$ & ${ }_{18}^{11}$ & $\begin{array}{l}29 \\
51 \\
\quad 80 \\
2.67\end{array}$ & $\begin{array}{l}17 \\
35 \\
52 \\
.54\end{array}$ & ${ }_{33}^{3}{ }_{38}$. & $\begin{array}{c}684 \\
(519)\end{array}$ \\
\hline $1971-72$ & $\begin{array}{l}\text { CLAREMONT } \\
\text { JEKI GIO }\end{array}$ & AA & & & $\begin{array}{l}46 \\
95 \\
1.1 \\
1.93\end{array}$ & $\begin{array}{l}37 \\
44 \\
\quad 81 \\
\quad .79\end{array}$ & $\begin{array}{l}37 \\
58 \\
\quad 95 \\
\quad .81\end{array}$ & $\begin{array}{l}23 \\
58 \\
\quad 91 \\
\quad .83\end{array}$ & $\begin{array}{l}23 \\
72 \\
95 \\
1.19\end{array}$ & $\begin{array}{l}40 \\
26 \\
{ }_{66} \\
.72\end{array}$ & $\begin{array}{l}17 \\
58 \\
\quad 75 \\
4.17\end{array}$ & $\begin{array}{l}21 \\
21 \\
42 \\
\quad .53\end{array}$ & ${ }^{21}{ }_{27}$ & $\begin{array}{c}713 \\
(569)\end{array}$ \\
\hline $1972-73$ & $\begin{array}{l}\text { CLAREMONT } \\
\text { JERI CIO }\end{array}$ & $A A$ & & & $\begin{array}{l}39 \\
82 \\
121 \\
1.41\end{array}$ & $\begin{array}{l}41 \\
77 \\
118 \\
.84\end{array}$ & $\begin{array}{l}31 \\
57 \\
\quad 83 \\
1.09\end{array}$ & $\begin{array}{l}32 \\
52 \\
\quad 84 \\
\quad .88\end{array}$ & $\begin{array}{l}31 \\
50 \\
{ }_{81} \\
\quad .89\end{array}$ & $\begin{array}{l}35 \\
44 \\
79 \\
.83\end{array}$ & $\begin{array}{l}25 \\
26 \\
\quad 51 \\
\quad .77\end{array}$ & $\begin{array}{l}16 \\
23 \\
39 \\
.52\end{array}$ & ${ }_{32}^{11}$ & $\begin{array}{c}693 \\
(571)\end{array}$ \\
\hline $1973-74$ & $\begin{array}{l}\text { CLAREMONTT } \\
\text { JERI CHO }\end{array}$ & MA & & & $\begin{array}{l}43 \\
2124 \\
124\end{array}$ & $\begin{array}{l}37 \\
70 \\
107\end{array}$ & $\begin{array}{l}30 \\
74 \\
304\end{array}$ & $\begin{array}{l}30 \\
44 \\
{ }^{74}\end{array}$ & $\begin{array}{l}38 \\
42 \\
{ }_{80}\end{array}$ & $\begin{array}{r}7 \\
47 \\
54\end{array}$ & ${ }_{86}^{45}$ & $\begin{array}{l}24 \\
37\end{array}$ & $\ddot{17}{ }_{17}$ & $\begin{array}{c}687 \\
(543)\end{array}$ \\
\hline \multicolumn{5}{|c|}{$\begin{array}{l}\text { Average Ratio to } 1970 / 71 \\
\text { Projections to } 1973 / 74 \\
\text { Arorigo Ratios to } 1972 / 73 \\
\text { Projoctions to } 1973 / 74\end{array}$} & $\begin{array}{l}1.16 \\
108 \\
12.29 \\
120\end{array}$ & $103^{887}$ & $8 \dot{5}^{90}$ & $\begin{array}{l}1,69 \\
89 \\
99^{.99}\end{array}$ & $\begin{array}{l}60^{77} \\
80^{-85}\end{array}$ & $5 i^{75}$ & $\begin{array}{l}1.73 \\
89 \\
1.94\end{array}$ & $\begin{array}{l}74^{83} \\
.84\end{array}$ & $\begin{array}{l}6 \dot{3}^{85} \\
6 \dot{8}^{78}\end{array}$ & $\begin{array}{c}723 \\
8 \\
826\end{array}$ \\
\hline
\end{tabular}


THE COHORT SURVIVAL METHOD

MIXED STRATUM - LUCEA

-Interpolated by Corputer

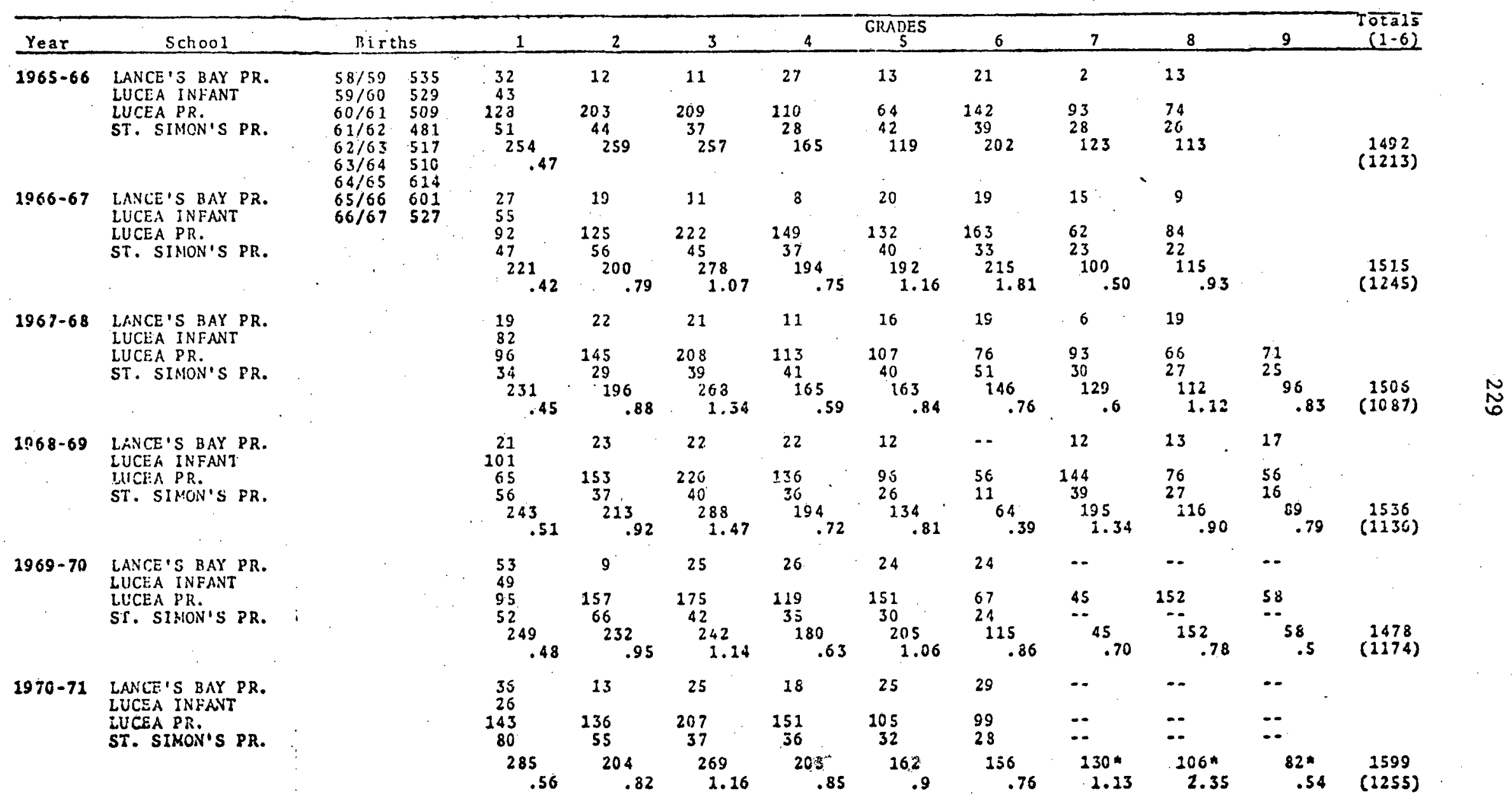


THE COHORT SURVIVAL METHOD

MIXED STRATUM - LUCEA - cont'd

*Interpolated by Compuzer

\begin{tabular}{ll} 
Year & \multicolumn{1}{c}{ School } \\
\hline $1971-72$ & LANCE'S BAY PR. \\
& LUCEA INFANT \\
& LUCEA PR. \\
& ST SIMON'S PR. \\
RUSEA HIGI!
\end{tabular}

1972-73 LANCE'S BAY PR. LUCEA INFANT LUCEA PR. ST. STRON'S

RUSEA JR. SEC.

9973-74 LANCI'S BAY FR. LUCEA INEANT LUCEA FR.

ST STMON'S PR.

RUSEA HIGil

RUSEA JR. SEC.

Average Ratio to $1970 / 71$

Projections to $1973 / 74$

Average Ratio to $1972 / 73$

Births

16

$\begin{array}{llll}36 & 16 & 13 & 22\end{array}$

$60 \quad 76$

$\begin{array}{ccc}218 & 279 & 315 \\ .36 & .98 & 1.54\end{array}$

32

$5 \quad 14 \quad 25$

37
78
58

78
58

$\begin{array}{lll}198 & 277 & 308 \\ .33 & 1.27 & 1.10\end{array}$

$\begin{array}{llll}40 & 10 & 31 & 24\end{array}$

39

$\begin{array}{rrr}42 & 428 & 160 \\ & 48 & 27\end{array}$

254

$257^{.48} \quad 22 \dot{4}^{.87}$

1.24
278
1.26

$219 \dot{7}^{71}$

$287^{95}$

$17 \dot{2}^{92}$

$14 \dot{6}^{85}$

178
1.22

$219^{.67}$
$232^{212}$

2758

1961 
THE COHORT SURVIVAL METHOD

MIXED STRATUM - GREEN ISLAND

AA- ALL AGE

\begin{tabular}{|c|c|c|c|c|c|c|c|c|c|c|c|c|c|}
\hline Year & School & Birt & & 1 & 2 & 3 & 4 & $\begin{array}{c}\text { CRADES } \\
-5 \\
\end{array}$ & 6 & 7 & 8 & 9 & $\begin{array}{r}\text { Totals } \\
(1-6)\end{array}$ \\
\hline $1965-66$ & $\begin{array}{l}\text { PELL RIVER PR. } \\
\text { CAIE VALLEY AA. } \\
\text { CHURCIILL PR. } \\
\text { COVE PR. } \\
\text { GREEN ISLAND PR. }\end{array}$ & $\begin{array}{l}58 / 59 \\
59 / 60 \\
60 / 61 \\
61 / 62 \\
62 / 63 \\
63 / 64 \\
64 / 65 \\
65 / 66\end{array}$ & $\begin{array}{r}.443 \\
439 \\
430 \\
414 \\
386 \\
336 \\
348 \\
337\end{array}$ & $\begin{array}{r}65 \\
83 \\
50 \\
46 \\
101 \\
\quad 345 \\
\quad .78\end{array}$ & $\begin{array}{l}50 \\
94 \\
51 \\
36 \\
89 \\
320\end{array}$ & $\begin{array}{l}39 \\
80 \\
37 \\
24 \\
62 \\
242\end{array}$ & $\begin{array}{l}37 \\
54 \\
34 \\
13 \\
46 \\
184\end{array}$ & $\begin{array}{c}34 \\
73 \\
18 \\
7 \\
48 \\
180\end{array}$ & $\begin{array}{l}52 \\
43 \\
17 \\
36 \\
46 \\
194\end{array}$ & $\begin{array}{c}29 \\
59 \\
17 \\
9 \\
35 \\
149\end{array}$ & $\begin{array}{l}27 \\
49 \\
39 \\
12 \\
34 \\
162\end{array}$ & & $\begin{array}{c}1775 \\
(1465)\end{array}$ \\
\hline 1966-67 & $\begin{array}{l}\text { PELL RIVER PR. } \\
\text { CAVE VALLEY AA } \\
\text { CHURCHILL PR: } \\
\text { COVE PR. } \\
\text { GREEN ISLAND PR. }\end{array}$ & $66 / 67$ & 353 & $\begin{array}{l}52 \\
77 \\
69 \\
46 \\
95 \\
339 \\
\quad .77\end{array}$ & $\begin{array}{c}70 \\
91 \\
68 \\
31 \\
136 \\
\quad 396 \\
\quad 1.15\end{array}$ & $\begin{array}{l}41 \\
97 \\
43 \\
19 \\
91 \\
291 \\
\quad .91\end{array}$ & $\begin{array}{l}36 \\
77 \\
26 \\
18 \\
55 \\
212 \\
\quad .88\end{array}$ & $\begin{array}{l}41 \\
88 \\
22 \\
11 \\
48 \\
210 \\
1.14\end{array}$ & $\begin{array}{l}46 \\
36 \\
16 \\
25 \\
47 \\
\quad 172 \\
\quad .95\end{array}$ & $\begin{array}{l}35 \\
52 \\
11 \\
21 \\
36 \\
155 \\
\quad .80\end{array}$ & $\begin{array}{l}29 \\
73 \\
27 \\
14 \\
18 \\
161 \\
1.08\end{array}$ & & $\begin{array}{c}1936 \\
(1620)\end{array}$ \\
\hline $1967-68$ & $\begin{array}{l}\text { PELL RIVER PR. } \\
\text { CAVE VALLEY AA } \\
\text { CHURCIILL PR. } \\
\text { COVE PR. } \\
\text { GREEN ISLAND PR. }\end{array}$ & & & $\begin{array}{r}76 \\
102 \\
68 \\
48 \\
122 \\
\quad 416 \\
\quad .97\end{array}$ & $\begin{array}{l}55 \\
83 \\
49 \\
35 \\
75 \\
297 \\
\quad .88\end{array}$ & $\begin{array}{r}50 \\
92 \\
58 \\
27 \\
110 \\
\quad 337 \\
\quad .85\end{array}$ & $\begin{array}{l}31 \\
97 \\
16 \\
20 \\
91 \\
255 \\
\quad .91\end{array}$ & $\begin{array}{l}16 \\
60 \\
5 \\
15 \\
54 \\
150.70 \\
\quad .70\end{array}$ & $\begin{array}{l}\overline{-} \\
32 \\
20 \\
\overline{13} \\
\quad 65 \\
\quad .31\end{array}$ & $\begin{array}{l}59 \\
62 \\
28 \\
28 \\
39 \\
216 \\
1.25\end{array}$ & $\begin{array}{l}34 \\
36 \\
11 \\
22 \\
40 . \\
149.96 \\
\quad .96\end{array}$ & $\begin{array}{l}23 \\
67 \\
8 \\
24 \\
22 \\
144 \\
\quad .89\end{array}$ & $\begin{array}{c}2029 \\
(1607)\end{array}$ \\
\hline $1968-69$ & $\begin{array}{l}\text { FELL RIVER PR. } \\
\text { CAVE VALIEY AA } \\
\text { CHURCHILL PR. } \\
\text { COVE PR. } \\
\text { GREEN ISLAND PR. }\end{array}$ & 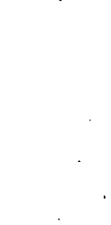 & & $\begin{array}{c}58 \\
87 \\
95 \\
48 \\
138 \\
426 \\
\quad 1.03\end{array}$ & $\begin{array}{r}71 \\
105 \\
48 \\
27 \\
103 \\
354 \\
\quad .85\end{array}$ & $\begin{array}{l}54 \\
83 \\
39 \\
27 \\
76 \\
279 \\
\quad .94\end{array}$ & $\begin{array}{r}24 \\
85 \\
24 \\
20 \\
130 \\
284 \\
\quad .84\end{array}$ & $\begin{array}{l}21 \\
72 \\
23 \\
15 \\
74 \\
205 \\
\quad .8\end{array}$ & $\begin{array}{c}27 \\
40 \\
7 \\
6 \\
31 \\
111 \\
\quad .74\end{array}$ & $\begin{array}{l}33 \\
68 \\
37 \\
24 \\
51 \\
213 \\
3.28\end{array}$ & $\begin{array}{l}29 \\
47 \\
11 \\
31 \\
31 \\
149 \\
\quad .69\end{array}$ & $\begin{array}{l}25 \\
29 \\
12 \\
25 \\
30 \\
122 \\
\quad .82\end{array}$ & $\begin{array}{r}2133 \\
\left(1650^{\circ}\right.\end{array}$ \\
\hline $1969-70$ & $\begin{array}{l}\text { PELL RIVER PR. } \\
\text { CAVE VALLYAA } \\
\text { CHURCIILL PR. } \\
\text { COVE PR. } \\
\text { GREEN ISLAND PR. } \\
\end{array}$ & . & & $\begin{array}{c}65 \\
140 \\
89 \\
53 \\
121 \\
468 \\
1.21\end{array}$ & $\begin{array}{l}58 \\
65 \\
66 \\
43 \\
148 \\
380 \\
.89\end{array}$ & $\begin{array}{c}64 \\
104 \\
38 \\
49 \\
103 \\
358 \\
1.01\end{array}$ & $\begin{array}{l}41 \\
83 \\
27 \\
18 \\
65 \\
234 \\
.84\end{array}$ & $\begin{array}{r}20 \\
51 \\
19 \\
8 \\
107 \\
205 \\
\quad .72\end{array}$ & $\begin{array}{l}10 \\
20 \\
32 \\
5 \\
21 \\
104 \\
.51\end{array}$ & $\begin{array}{c}18 \\
84 \\
\cdots \\
220 \\
322 \\
2.9\end{array}$ & \begin{tabular}{c}
4 \\
37 \\
\hdashline- \\
235 \\
276 \\
1.30
\end{tabular} & $\begin{array}{l}3 \\
13 \\
=- \\
212 \\
227 \\
2.52\end{array}$ & $\begin{array}{r}2574 \\
(1749)\end{array}$ \\
\hline
\end{tabular}


THE COHORT SURVIVAL METHOD

MIXED STRATUM - GREEN ISLAND - cont'd

\begin{tabular}{|c|c|c|c|c|c|c|c|c|c|c|c|c|}
\hline Year & School & Births & 1 & 2 & 3 & 4 & $\begin{array}{c}\text { GPADES } \\
5\end{array}$ & 6 & 7 & 8 & 9 & $\begin{array}{r}\text { Totals } \\
(1-6) \\
\end{array}$ \\
\hline $1970-71$ & $\begin{array}{l}\text { PELL RIVER PR. } \\
\text { CAVE VALLEY AA } \\
\text { CHURCHILL PR. } \\
\text { COVE PR. } \\
\text { GRFEN ISLAND PR. }\end{array}$ & & $\begin{array}{r}9 \\
310 \\
92 \\
29 \\
115 \\
355 \\
1.06\end{array}$ & $\begin{array}{r}54 \\
134 \\
67 \\
52 \\
124 \\
431 \\
\quad .92\end{array}$ & $\begin{array}{c}51 \\
89 \\
47 \\
50 \\
143 \\
\quad 380 \\
1.0\end{array}$ & $\begin{array}{l}63 \\
96 \\
41 \\
32 \\
80 \\
\quad 312 \\
\quad .87\end{array}$ & $\begin{array}{l}42 \\
71 \\
29 \\
12 \\
44 \\
198 \\
\quad .85\end{array}$ & $\begin{array}{l}13 \\
.28 \\
37 \\
6 \\
30 \\
114 \\
\quad .56\end{array}$ & $\begin{array}{c}12 \\
63 \\
\cdots \\
\cdots \\
243 \\
318 \\
3.06\end{array}$ & $\begin{array}{c}\overline{55} \\
\because- \\
257 \\
\quad 312 \\
\quad .97\end{array}$ & $\begin{array}{c}\ddot{21} \\
\because- \\
270 \\
291 \\
\quad 1.05\end{array}$ & $\begin{array}{r}2711 \\
(1790)\end{array}$ \\
\hline $1971-72$ & $\begin{array}{l}\text { PELL RIVER PR. } \\
\text { CAVE VALLEY AA } \\
\text { CIURCIILL PR. } \\
\text { COVE PR. } \\
\text { GREEN ISLAND PR. }\end{array}$ & . & $\begin{array}{c}61 \\
79 \\
95 \\
24 \\
106 \\
365 \\
\quad 1.05\end{array}$ & $\begin{array}{r}43 \\
118 \\
67 \\
29 \\
112 \\
369 \\
1.04 ?\end{array}$ & $\begin{array}{r}55 \\
135 \\
55 \\
53 \\
116 \\
\quad 414 \\
\quad .96\end{array}$ & $\begin{array}{c}47 \\
94 \\
54 \\
47 \\
143 \\
\quad 385 \\
\quad 1.01\end{array}$ & $\begin{array}{l}57 \\
125 \\
37 \\
18 \\
79 \\
316 \\
1.01\end{array}$ & $\begin{array}{c}33 \\
6 \\
42 \\
7 \\
35 \\
123 \\
\quad .62\end{array}$ & $\begin{array}{c}- \\
66 \\
\therefore \\
208 \\
274 \\
2.4\end{array}$ & $\begin{array}{c}39 \\
\therefore- \\
240 \\
279 \\
\quad .87\end{array}$ & $\begin{array}{l}-8 \\
14 \\
\therefore- \\
297 \\
311 \\
\\
.99\end{array}$ & $\begin{array}{c}2836 \\
(1972)\end{array}$ \\
\hline $1972-73$ & $\begin{array}{l}\text { FELL RIVER PR. } \\
\text { CAVE VALLEY AA } \\
\text { CHURCIILL FR. } \\
\text { COVE FR. } \\
\text { GREEN ISLAND PR. }\end{array}$ & & $\begin{array}{r}77 \\
134 \\
95 \\
34 \\
119 \\
439 \\
\quad 1.3\end{array}$ & $\begin{array}{r}52 \\
73 \\
67 \\
28 \\
106 \\
\quad 326 \\
\quad .89\end{array}$ & $\begin{array}{r}50 \\
103 \\
55 \\
27 \\
100 \\
335 \\
.91\end{array}$ & $\begin{array}{r}60 \\
215 \\
54 \\
53 \\
112 \\
394 \\
\quad .93\end{array}$ & $\begin{array}{r}49 \\
62 \\
62 \\
31 \\
275 \\
\quad 304 \\
\quad .78\end{array}$ & $\begin{array}{l}69 \\
38 \\
42 \\
8 \\
34 \\
191 \\
\quad .62\end{array}$ & $\begin{array}{l}\overline{55} \\
-- \\
-- \\
208 \\
263 \\
\quad 2.14\end{array}$ & $\begin{array}{c}\angle 2 \\
\cdots \\
240 \\
282 \\
\quad 1.03\end{array}$ & $\begin{array}{l}-- \\
23 \\
-- \\
297 \\
320 \\
2.15\end{array}$ & $\begin{array}{c}2854 \\
(1989)\end{array}$ \\
\hline $1973-74$ & $\begin{array}{l}\text { PELI. RIVER PR. } \\
\text { CAVE VALLEY AA } \\
\text { CIURCHILL PR. } \\
\text { COVE PR. } \\
\text { GREEN ISLAND PR. }\end{array}$ & & $\begin{array}{c}70 \\
99 \\
87 \\
26 \\
203 \\
\quad 385\end{array}$ & $\begin{array}{c}50 \\
100 \\
115 \\
35 \\
119 \\
425\end{array}$ & $\begin{array}{c}49 \\
92 \\
54 \\
28 \\
105 \\
328\end{array}$ & $\begin{array}{l}51 \\
95 \\
71 \\
30 \\
86 \\
333\end{array}$ & $\begin{array}{c}64 \\
91 \\
47 \\
55 \\
106 \\
363\end{array}$ & $\begin{array}{l}55 \\
41 \\
35 \\
10 \\
69 \\
210\end{array}$ & $\begin{array}{c}14 \\
53 \\
\because- \\
263 \\
330\end{array}$ & $\begin{array}{c}-2 \\
\therefore 6 \\
\therefore- \\
193 \\
239\end{array}$ & $\begin{array}{c}\ddot{28} \\
\therefore \\
225 \\
253\end{array}$ & $\begin{array}{c}2866 \\
(2044)\end{array}$ \\
\hline $\begin{array}{l}\text { Average } \\
\text { Projecti } \\
\text { Average } \\
\text { Projecti }\end{array}$ & $\begin{array}{l}\text { Ratio to } 1970 / 71 \\
\text { ons to } 1973 / 74 \\
\text { Ratio to } 1972 / 73 \\
\text { ons to } 1973 / 74\end{array}$ & & $\begin{array}{l}37 \dot{5}^{97} \\
39.02\end{array}$ & $35 \dot{3}^{94}$ & $33 i^{94}$ & $31 .^{90}$ & $24 \dot{3}^{84}$ & $\begin{array}{l}1486 \\
169^{.62}\end{array}$ & $\begin{array}{l}2.26 \\
335 \\
2.26 \\
383^{26}\end{array}$ & $\begin{array}{l}3.00 \\
335^{.99} \\
379^{-9}\end{array}$ & $\begin{array}{l}1.07 \\
358 \\
1.07 \\
406\end{array}$ & $\begin{array}{l}2768 \\
3051\end{array}$ \\
\hline
\end{tabular}


THE COHORT SURVIVAL METHOD

MIXED STRATUM - RIVERSIDE-DIAS

\begin{tabular}{|c|c|c|c|c|c|c|c|c|c|c|c|c|c|}
\hline Year & School & Birt & & 1 & 2 & 3 & 4 & $\begin{array}{c}\text { TPADES } \\
5\end{array}$ & 6 & 7 & 8 & 9 & $\begin{array}{r}\text { Totais } \\
(1-6) \\
\end{array}$ \\
\hline $1965-66$ & $\begin{array}{l}\text { CACOON ALL ACE } \\
\text { MIDDIESEX CNR. PR. } \\
\text { RIVERSIDE ALL AGE }\end{array}$ & $\begin{array}{l}58 / 5 n \\
59 / 60 \\
60 / 61 \\
61 / 62 \\
62 / 63 \\
63 / 64 .\end{array}$ & $\begin{array}{l}224 \\
222 \\
256 \\
226 \\
178 \\
242 \\
262\end{array}$ & $\begin{array}{l}85 \\
84 \\
110 \\
\quad 279 \\
\quad 1.25\end{array}$ & $\begin{array}{c}73 \\
118 \\
87 \\
278\end{array}$ & $\begin{array}{c}58 \\
118 \\
113 \\
269\end{array}$ & $\begin{array}{l}64 \\
43 \\
86 \\
193\end{array}$ & $\begin{array}{l}54 \\
55 \\
89 \\
198\end{array}$ & $\begin{array}{l}41 \\
72 \\
74 \\
187\end{array}$ & $\begin{array}{l}34 \\
47 \\
50 \\
131\end{array}$ & $\begin{array}{c}49 \\
107 \\
72 \\
228\end{array}$ & & $\begin{array}{r}1783 \\
(2424\end{array}$ \\
\hline $1966-67$ & $\begin{array}{l}\text { CACOON ALL AGE } \\
\text { AIDULESEX CNR. PR. } \\
\text { RIVERSIDE ALL AGE }\end{array}$ & $\begin{array}{l}64 / 65 \\
65 / 66 \\
66 / 67\end{array}$ & $\begin{array}{l}263 \\
254 \\
245\end{array}$ & $\begin{array}{l}54 \\
100 \\
117 \\
271 \\
\quad 1.22\end{array}$ & $\begin{array}{l}67 \\
107 \\
88 \\
262 \\
\quad .94\end{array}$ & $\begin{array}{l}70 \\
97 \\
103 \\
\quad 270 \\
\quad .97\end{array}$ & $\begin{array}{r}59 \\
110 \\
78 \\
\quad 247 \\
\quad .85\end{array}$ & $\begin{array}{l}69 \\
60 \\
88 \\
223 \\
1.16\end{array}$ & $\begin{array}{l}50 \\
22 \\
84 \\
156 \\
\quad .79\end{array}$ & $\begin{array}{l}37 \\
39 \\
56 \\
132 \\
\quad .71\end{array}$ & $\begin{array}{l}43 \\
124 \\
67 \\
234 \\
1.79\end{array}$ & & $\begin{array}{c}1795 \\
(1429)\end{array}$ \\
\hline 1967-08 & $\begin{array}{l}\text { CACOON ALL ACE } \\
\text { MICULESEX CNR. PR. } \\
\text { RIVEREIDE ALL AGE: }\end{array}$ & & & $\begin{array}{l}77 \\
118 \\
117 \\
312 \\
1.22\end{array}$ & $\begin{array}{c}58 \\
95 \\
123 \\
276 \\
\quad 1.02\end{array}$ & $\begin{array}{c}65 \\
114 \\
91 \\
270 \\
\quad 1.03\end{array}$ & $\begin{array}{l}78 \\
81 \\
88 \\
247 \\
\quad .91\end{array}$ & $\begin{array}{r}63 \\
71 \\
102 \\
236 \\
\quad .96\end{array}$ & $\begin{array}{l}29 \\
44 \\
58 \\
131 \\
.59\end{array}$ & $\begin{array}{l}45 \\
42 \\
71 \\
158 \\
1.01\end{array}$ & $\begin{array}{l}35 \\
20 \\
37 \\
\quad 92 \\
\quad .70\end{array}$ & $\begin{array}{l}20 \\
91 \\
30 \\
141 \\
.60\end{array}$ & $\begin{array}{c}1863 \\
(1472)\end{array}$ \\
\hline 1968.69 & $\begin{array}{l}\text { CACOON ALL AGE } \\
\text { MIDNLESEX CNR. PR! } \\
\text { RIVERSIDE, ALL AGE: }\end{array}$ & & & $\begin{array}{c}74 \\
82 \\
117 \\
273 \\
\quad 1.21\end{array}$ & $\begin{array}{r}41 \\
99 \\
123 \\
263 \\
\quad .84\end{array}$ & $\begin{array}{l}45 \\
82 \\
91 \\
\quad 218 \\
\quad .79\end{array}$ & $\begin{array}{l}43 \\
98 \\
90 \\
231 \\
\quad .86\end{array}$ & $\begin{array}{l}21 \\
56 \\
102 \\
\quad 179 \\
\quad .72\end{array}$ & $\begin{array}{l}6 \\
61 \\
61 \\
128 \\
\quad .54\end{array}$ & $\begin{array}{l}42 \\
80 \\
71 \\
193 \\
1.47\end{array}$ & $\begin{array}{l}17 \\
44 \\
56 \\
117 . \\
.74\end{array}$ & $\begin{array}{l}13 \\
65 \\
15 \\
93 \\
1.01\end{array}$ & $\begin{array}{c}1695 \\
(1292)\end{array}$ \\
\hline $1969-70$ & $\begin{array}{l}\text { CACOON ALL AGE } \\
\text { MIDDLESEX CNR. PR. } \\
\text { RIVERSIDE AIL AGE. }\end{array}$ & & & $\begin{array}{l}70 \\
60 \\
124 \\
260 \\
1.46\end{array}$ & $\begin{array}{r}72 \\
105 \\
107 \\
284 \\
1.04\end{array}$ & $\begin{array}{c}79 \\
99 \\
110 \\
288 \\
\quad 1.10\end{array}$ & $\begin{array}{l}61 \\
92 \\
77 \\
230 \\
\quad 1.06\end{array}$ & $\begin{array}{l}43 \\
61 \\
49 \\
153 \\
.66\end{array}$ & $\begin{array}{l}44 \\
56 \\
43 \\
143 \\
.80\end{array}$ & $\begin{array}{l}65 \\
45 \\
108 \\
\quad 218 \\
\quad 1.70\end{array}$ & $\begin{array}{l}54 \\
86 \\
48 \\
188 \\
\quad .97\end{array}$ & $\begin{array}{c}37 \\
73 \\
14 \\
124 \\
1.06\end{array}$ & $\begin{array}{c}1888 \\
(1358)\end{array}$ \\
\hline $1970-71$ & $\begin{array}{l}\text { CACOON ALI. AGE } \\
\text { MI DDLESEX CNR. PR. } \\
\text { RIVERSIDE ALL AGE }\end{array}$ & & & $\begin{array}{r}76 \\
90 \\
114 \\
280 \\
1.16\end{array}$ & $\begin{array}{c}63 \\
77 \\
123 \\
263 \\
1.01\end{array}$ & $\begin{array}{l}31 \\
102 \\
105 \\
238 \\
\quad .84\end{array}$ & $\begin{array}{r}73 \\
108 \\
101 \\
282 \\
\quad .98\end{array}$ & $\begin{array}{l}35 \\
79 \\
54 \\
168 \\
\quad .73\end{array}$ & $\begin{array}{l}36 \\
50 \\
45 \\
132 \\
\quad .86\end{array}$ & $\begin{array}{l}61 \\
-- \\
90 \\
151 \\
1.06\end{array}$ & $\begin{array}{l}58 \\
35 \\
93 \\
\quad .43\end{array}$ & $\begin{array}{l}31 \\
-2 \\
20 \\
51 \\
\quad .27\end{array}$ & $\begin{array}{c}1658 \\
(1363)\end{array}$ \\
\hline $\begin{array}{l}1971=72 \\
\therefore .\end{array}$ & $\begin{array}{l}\text { CACOON ALL AGE } \\
\text { MIDDLESEX CNR. PR. } \\
\text { RIVERSIDE ALL AGE }\end{array}$ & & & $\begin{array}{c}83 \\
85 \\
127 \\
295 \\
1.12\end{array}$ & $\begin{array}{l}47 \\
211 \\
92 \\
250 \\
.89\end{array}$ & $\begin{array}{c}54 \\
77 \\
209 \\
240 \\
\quad .91\end{array}$ & $\begin{array}{c}51 \\
101 \\
93 \\
245 \\
1.03\end{array}$ & $\begin{array}{l}47 \\
83 \\
58 \\
18.4 \\
\quad .67\end{array}$ & $\begin{array}{l}55 \\
44 \\
47 \\
146 \\
.87\end{array}$ & $\begin{array}{c}43 \\
105 \\
148 \\
1.12\end{array}$ & $\begin{array}{l}28 \\
31 \\
59 \\
\quad 39\end{array}$ & $\begin{array}{l}43 \\
-15 \\
\quad 58 \\
\quad .62\end{array}$ & $\begin{array}{c}1629 \\
(1364)\end{array}$ \\
\hline
\end{tabular}


THE COHORT SURVIVAL METHOD

MIXED STRATUM - RIVERSIDE-DIAS - cont'd

\begin{tabular}{|c|c|c|c|c|c|c|c|c|c|c|c|c|}
\hline Yesr & School & Births & 1 & 2 & 3 & 4 & $\begin{array}{c}\text { GKADES } \\
5 \\
\end{array}$ & 6 & 7 & 8 & 9 & $\begin{array}{r}\text { Totals } \\
(1-6) \\
\end{array}$ \\
\hline $1972-73$ & $\begin{array}{l}\text { CACOON ALL AGE } \\
\text { MIDDLESEX CNR. PR. } \\
\text { RIVERSIDE ALL AGF }\end{array}$ & & $\begin{array}{c}89 \\
70 \\
116 \\
275 \\
1.08\end{array}$ & $\begin{array}{l}80 \\
102 \\
126 \\
308 \\
1.04\end{array}$ & $\begin{array}{l}67 \\
106 \\
101 \\
274 \\
1.10\end{array}$ & $\begin{array}{l}58 \\
76 \\
119 \\
253 \\
1.05\end{array}$ & $\begin{array}{l}52 \\
82 \\
68 \\
202 \\
.82\end{array}$ & $\begin{array}{l}43 \\
64 \\
50 \\
157 \\
\quad .84\end{array}$ & \begin{tabular}{l}
62 \\
\hdashline- \\
114 \\
$\quad 176$ \\
$\quad 1.21$
\end{tabular} & $\begin{array}{l}34 \\
-- \\
30 \\
64 \\
.43\end{array}$ & $\begin{array}{l}32 \\
26 \\
58 \\
.98\end{array}$ & $\begin{array}{c}1767 \\
(1469)\end{array}$ \\
\hline $\begin{array}{l}1973-74 \\
\vdots\end{array}$ & $\begin{array}{l}\text { CACCON ALL AGE } \\
\text { MIDDLESEX CNR. PR: } \\
\text { RIVERS IDE ALL AGE } \\
-\end{array}$ & & $\begin{array}{c}79 \\
91 \\
108 \\
278\end{array}$ & $\begin{array}{r}83 \\
93 \\
106 \\
282\end{array}$ & $\begin{array}{l}78 \\
103 \\
109 \\
290\end{array}$ & $\begin{array}{l}63 \\
99 \\
85 \\
247\end{array}$ & $\begin{array}{l}47 \\
73 \\
63 \\
183\end{array}$ & $\begin{array}{l}32 \\
68 \\
53 \\
153\end{array}$ & $\begin{array}{l}55 \\
7- \\
79 \\
134\end{array}$ & $\begin{array}{l}56 \\
17 \\
73\end{array}$ & $\begin{array}{l}36 \\
23 \\
59\end{array}$ & $\begin{array}{c}1699 \\
(1433)\end{array}$ \\
\hline $\begin{array}{l}\text { Average } \\
\text { Projecti } \\
\text { Average } \\
\text { Projecti }\end{array}$ & $\begin{array}{l}\text { Ratio to } 1970 / 71 \\
\text { ons to } 1973 / 74 \\
\text { Ratio to } 1972 / 73 \\
\text { ons to } 1973 / 74\end{array}$ & & $\begin{array}{l}2 \frac{1}{295} \dot{3}^{25} \\
\frac{1}{285^{22}}\end{array}$ & $28 \dot{4}^{97}$ & $\begin{array}{l}27 \dot{0}^{95} \\
26 \dot{5}^{96}\end{array}$ & $\begin{array}{l}25 i^{93} \\
25 i^{93}\end{array}$ & $\begin{array}{l}21 \dot{3}^{85} \\
20 \dot{8}^{82}\end{array}$ & $\begin{array}{l}15 \dot{3}^{72} \\
15 \dot{8}^{76}\end{array}$ & $\begin{array}{l}1.19 \\
182 \\
1.28 \\
186\end{array}$ & $\begin{array}{l}16 \dot{9}^{93} \\
14 \dot{5}^{78}\end{array}$ & ${ }_{11 \dot{0}^{76}}^{12 \dot{7}^{74}}$ & $\begin{array}{l}1940 \\
1857^{\prime}\end{array}$ \\
\hline
\end{tabular}


THE COHORT SURVIVAL METHOD

TOTAL ENROLLMENT - HANOVER

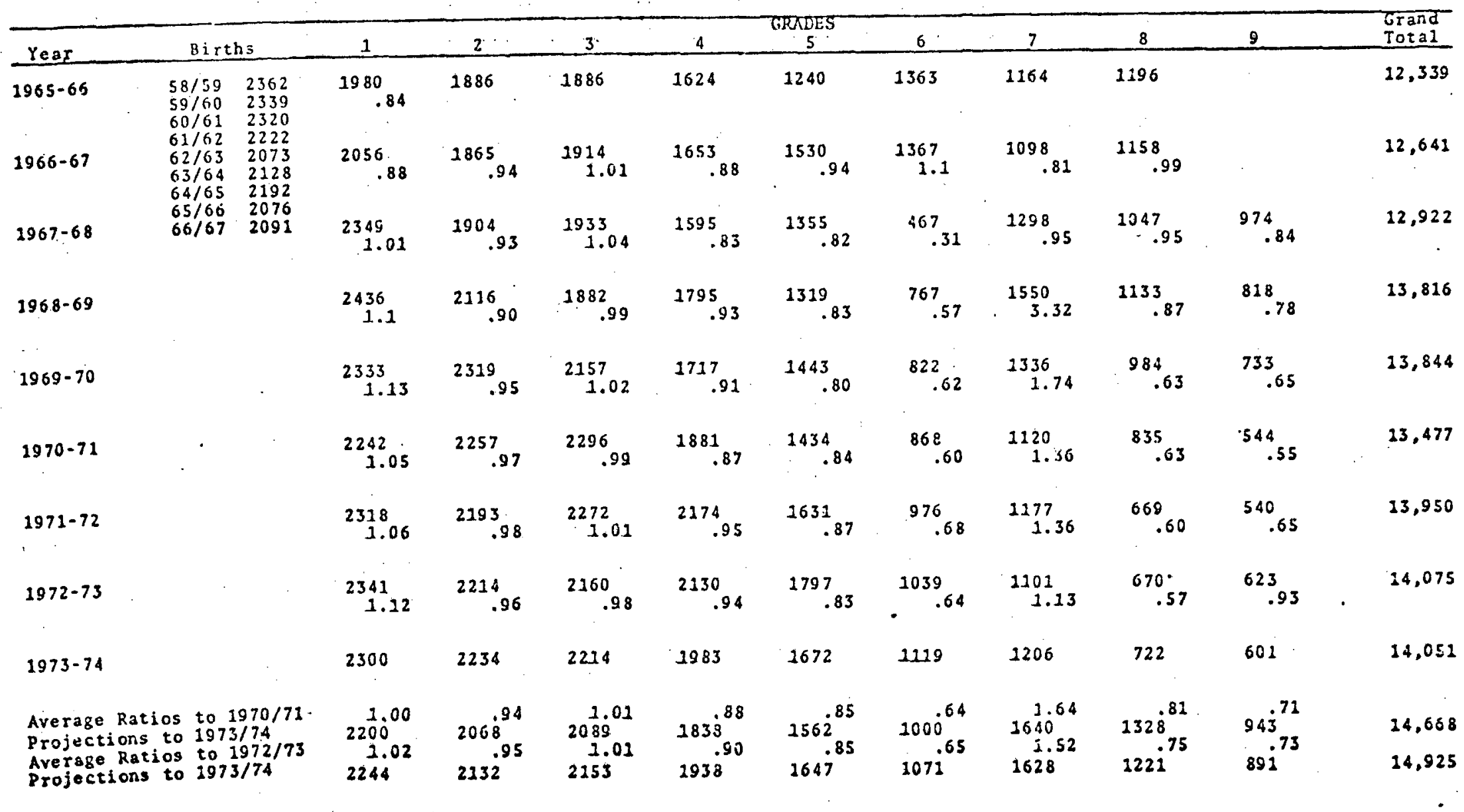


B I B L I O G R A P H Y 


\section{BIBLIOGRAPHY}

Armitage, P. and Smith, C. "The Development of Computable Models of the British Educational System and their Possible Uses," Mathematical Models in Educational Planning. Paris, France: Directorate for Scientific Affairs, Organization of Economic Co-operational Development, 1967.

Brooks, Kenneth Wayne. "Taxonomies of Data Sources Available for Comprehensive Educational P1anning," Ph.D. Dissertation, The Ohio State University, 1972.

Brown, B. W. and Savage, J. R. Methodological Studies in Educationa1 Attendance Frediction. Minneapolis, Minnesota: Department of Statistics, University of Minnesota, 1960.

Correa, Hector. Quantitative Methods of Educational Planning. Scranton, Pennsylvania: International Textbook Company, 1969.

Denham, Carolyn H. Probabilistic School Enrollment Predicticns Using Monte Carlo Computer Simulation--Final Report. Washington: ERIC Document Reproduction Service, ED 062 729, 1971.

Encyclopedia of Educational Research revised edition 1950, s.v. "Population Growt:h" by T. C. Holy and Willis A. Whitehead.

Gani, J. "Formulae for Projecting Enrollments and Degrees Awarded in Universities," Journal of the Royal Statistical Society, Series A, 126, Part 3, 1963, pp. 400-409.

Gordon, Shirley C., ed. A Century of West Indian Education. London: Longman Group Ltd., 1963.

Greenawalt, George J. and Mitchell, Donald P. Predicting School Enrol1ments. Cambridge, Mass.: ERIC Document Reproduction Service, ED 026 717, 1966.

Jaffe, A. J. Handbook of Statistical Procedures for Long Range Projections of Fublic School Enrollment. Washington: ERIC Document Reproduction Service, ED 058 668, 1969.

Jamaica, Department of Statistics: Statistical Abstract 1973. Statistical Yearbook of Jamaica, 1974. 
Five Year Independence P1an, 1963-1968--A Long Term Deve1opment Programme for Jamaica.

Joss, Charles C. "A Comparison of Two Selected Methods of Predicting Enrollments in Rapidly Growing School Districts," Ph.D. Dissertation, University of Iowa, 1969.

Larson, Knute G. and Strevell, Wallace H. "How Reliable are Enrollment Forecasts?" The School Executive, February 1952, pp. 65-68.

Liu, Bangnee Alfred. Estimating Future School Enrollments in Developing Countries-A Manual of Methodology. UNESCO, Paris: ERIC Document Reproduction Service, ED 078 545, 1966.

Mohrenweiser, Gary A. "A Markov Chain Model for Projecting School Enrollments," Ph.D. Dissertation, University of Minnesota, 1969.

Roberts, G. W.; Powell, Dorian; Sinclair, Sonja; Boland, Barbara and Hewitt, Iinda. Recent Population Movements in Jamaica. Kingston, Jamaica: The Herald Ltd., 1974.

Streve11, Wallace H. "School Plant Adequacy through Techniques of Estimating Future Enrol1ment," School Board Journal, March 1952, pp. 35-38.

Skutsch, Margaret and Hall, Diana. Delphi: Fotential Uses in Educational Planning. Washington: ERIC Document Reproduction Service, ED $084659,1973$.

Thonstad, T. "A Mathematical Model of the Norwegian Educational System," Mathematical Models in Educational Planning. Paris: Organization for Economic Co-operation and Development, 1967, pp. 125128.

Walsh; B. T. "The Effects of Alternative Population Trends on Economic Growth in Jamaica," Ph.D. Dissertation, University of Pittsburgh, 1970.

Wasik, John L. A Review and Critical Analysis of Models Used for Estimating Enrollments in Educational Systems. North Carolina: ERIC Document Reproduction Service, ED 059 545, 1971.

Webster, William J. "The Applicability of Selected Ratio and Least Squares Regression Analysis Techniques to the Prediction of Future Educational Attendance Patterns," Ph.D. Dissertation, Michigan State University, 1969.

"An Empirical Comparison of Selected Enrollment Projection Formulas." The Journal of Educational Research, Vol. 64, April 1971, pp. 375-381. 
Wong, Y. "Computer Simulation of Student Mobility Patterns," Journal of the Association for Educational Data Systems, 2,1969 , pp.

Zabrowski, Edward K. Student-Teacher Population Growth Mode1--Dynamod II. Washington: ERIC Document Reproduction Service, ED 015
556, 1967 .

Zimmer, John Francis. "An Evaluation of Four Methods of Enrollment Projection as Applied to a State College System." Ph.D. Dissertation, University of Minnesota, 1971. 